\title{
Regioselectively Nucleus and/or Side-Chain Fluorinated 2- (Phenanthryl)propionic Acids by an Effective Combination of Radical and Organometallic Chemistry
}

\author{
Renzo Ruzziconi,* Giacomo Ricci \\ Dipartimento di Chimica, Università di Perugia, Via elce di sotto, 8, I-06123 Perugia, Italy
}

Table of Contents:

\section{Product}

2-(6-fluorophenanthren-1-yl)propionic acid (1b)

2-(6,8-difluorophenanthr-1-yl)propionic acid (1c)

2-Fluoro-2-(phenanthr-1-yl)propionic acid (2a)

2-Fluoro-2-(6-fluorophenanthr-1-yl)propionic acid (2b)

2-(6,8-difluorophenanthr-1-yl)-2-fluoropropionic acid (2c)

2-(phenanthr-1-yl)-2,3,3,3-tetrafluoropropionic acid (3a)

2-(6-fluorophenanthr-1-yl)-2,3,3,3-tetrafluoropropionic

acid (3b)

2-(6,8-difluorophenanthr-1-yl)-2,3,3,3-tetrafluoropropionic $\operatorname{acid}(3 \mathbf{c})$

2-(1,3-difluorophenanthr-2-yl)-2-fluoropropionic acid (4a)

2-(8-bromo-1,3-difluorophenanthr-2-yl)-2-fluoropropionic acid (4b)

2-(1,3-difluorophenanthr-2-yl)-2,3,3,3-tetrafluoropropionic acid (5a)

2-(8-bromo-1,3-difluorophenanthr-2-yl)-2,3,3,3-tetrafluoropropionic acid $(\mathbf{5 b})$

ethyl 2-(6-fluorophenanthren-1-yl)propionate (11b)

ethyl 2-(6,8-difluorophenanthren-1-yl)propionate (11c)

1-Bromo-3,4-dihydrophenanthrene (12a)

1-Bromo-6-fluoro-3,4-dihydrophenanthrene (12b)

1-Bromo-6,8-difluoro-3,4-dihydrophenanthrene (12c)

Methyl 2-hydroxy-2-(phenanthr-1-yl)propionate (14a)

Methyl 2-hydroxy-2-(6-fluorophenanthren-1-yl)propionate (14b)

Ethyl 2-hydroxy-2-(phenanthren-1-yl)-3,3,3-trifluoropropionate (14c)

Ethyl 2-hydroxy-2-(6-fluorophenanthren-1-yl)-3,3,3-trifluoropropionate (14d)

Methyl 2-(6,8-difluoro-3,4-dihydrophenanthren-1-yl)-2-

hydroxypropionate (15a)

\section{${ }^{1}$ H NMR ${ }^{13}$ C NMR ${ }^{19}$ F NMR}

\begin{tabular}{|c|c|c|}
\hline S1 & S2 & S3 \\
\hline S4 & S5 & S6 \\
\hline S7 & S8 & S9 \\
\hline S10 & S11 & S12 \\
\hline S13 & S14 & S15 \\
\hline S16 & S17 & S18 \\
\hline S19 & $\mathbf{S 2 0}$ & S21 \\
\hline $\mathbf{S 2 2}$ & $\mathbf{S 2 3}$ & S24 \\
\hline S25 & S26 & S27 \\
\hline S28 & S29 & S30 \\
\hline S31 & S32 & S33 \\
\hline S34 & S35 & S36 \\
\hline S37 & S38 & S39 \\
\hline S40 & S41 & S42 \\
\hline S43 & S44 & \\
\hline S45 & S46 & S47 \\
\hline S48 & S49 & S50 \\
\hline S51 & S52 & \\
\hline S53 & S54 & S55 \\
\hline S56 & S57 & S58 \\
\hline S59 & S60 & S61 \\
\hline S62 & S63 & S64 \\
\hline
\end{tabular}


Ethyl 2-(6,8-difluoro-3,4-dihydrophenanthren-1-yl)-2-hydroxy3,3,3-trifluoropropionate (15b)

S65

S66

S67

Methyl 2-(8-bromo-1,3-difluoro-5,6-dihydrophenanthren-2-yl)-

2-hydroxypropionate (16a)

S68

S69

S70

Ethyl 2-(8-bromo-1,3-difluoro-5,6-dihydrophenanthren-2-yl)-2hydroxy-3,3,3-trifluoropropio-nate (16b)

S71 $\quad$ S72 $\quad$ S73

Methyl 2-(1,3-difluoro-5,6-dihydrophenanthren-2-yl)-2-

hydroxypropionate $(\mathbf{1 7 a})$

Ethyl 2-(1,3-difluoro-5,6-dihydrophenanthren-2-yl)-2-hydroxy3,3,3-trifluoropropionate $(\mathbf{1 7 b})$

Methyl 2-[1,3-difluoro-5,6-dihydro-8-(1-hydroxy-1-methoxycarbonylethyl)phenanthren-2-yl]-2-hydroxypropionate (18a)

6,8-Difluoro-3,4-dihydrophenanthrene (19)

8-Bromo-1,3-difluorophenanthrene (24)

Methyl 2-(6,8-difluorophenanthren-1-yl)-2-hydroxypropionate (25a)

Ethyl 2-(6,8-difluorophenanthren-1-yl)-2-hydroxy-3,3,3-trifluoropropionate $\mathbf{2 5 b})$

S74 S75 S76

S77 $\quad$ S78 $\quad$ S79

S80 $\quad$ S81 $\quad$ S82

S83 $\quad$ S84 $\quad$ S85

S86 $\quad$ S87 $\quad$ S88

Methyl 2-(1,3-difluorophenanthren-2-yl)-2-hydroxypropionate (26a)

Ethyl 2-(1,3-difluorophenanthren-2-yl)-2-hydroxy-3,3,3-trifluoropropionate (26b)

Methyl 2-[6,8-Difluoro-7-(1-hydroxy-1-methoxycarbonyl-

ethyl)phenanthren-1-yl]-2-hydroxy-propionate (27a)

1,3-Difluorophenanthrene (28)

Methyl 2-(8-bromo-1,3-difluorophenanthren-2-yl)-2-hydroxypropionate $(\mathbf{2 9 a})$

S89 $\quad$ S90 $\quad$ S91

S92 $\quad$ S93 $\quad$ S94

S95 $\quad$ S96 $\quad$ S97

S98 $\quad$ S99 $\quad$ S100

S101 S102 S103

S104 S105 S106

Ethyl 2-(8-Bromo-1,3-difluorophenanthren-2-yl)-2-hydroxy-

3,3,3-trifluoropropanoate (29b)

Methyl 2-fluoro-2-(phenanthren-1-yl)propionate (30a)

Methyl 2-fluoro-2-(6-fluorophenanthren-1-yl)propionate (30b)

Ethyl 2-(phenanthren-1-yl)-2,3,3,3-tetrafluoropropionate (30c)

Ethyl 2-(6-fluorophenanthren-1-yl)-2,3,3,3-tetrafluoropropionate $\mathbf{( 3 0 d )}$

Methyl 2-(6,8-difluorophenanthren-1-yl)-2-fluoropropionate (30e)

Ethyl 2-(6,8-difluorophenanthren-1-yl)-2,3,3,3-tetrafluoropropionate (30f)

$\mathbf{S 1 0 7} \mathbf{S 1 0 8} \quad \mathbf{S 1 0 9}$

S110 S111 S112

S113 S114 S115

S116 S117 S118

S119 S120 S121

S122 $\quad$ S123 $\quad$ S124

$\mathbf{S 1 2 5} \quad \mathbf{S 1 2 6} \quad \mathbf{S 1 2 7}$

$\mathbf{S 1 2 8} \quad \mathbf{S 1 2 9} \quad \mathbf{S 1 3 0}$

Methyl 2-(1,3-difluorophenanthren-2-yl)-2-fluoropropionate (31a)

S131 S132 S133

Methyl 2-(8-bromo-1,3-difluorophenanthren-2-yl)-2-fluoropropionate (31b)

S134 S135 S136

Ethyl 2-(1,3-difluorophenanthren-2-yl)-2,3,3,3-tetrafluoropropionate (31c)

S137 $\quad$ S138 $\quad$ S139

Ethyl 2-(8-bromo-1,3-difluorophenanthren-2-yl)-2,3,3,3-tetra-

fluoropropionate (31d)

S140 S141 S142 

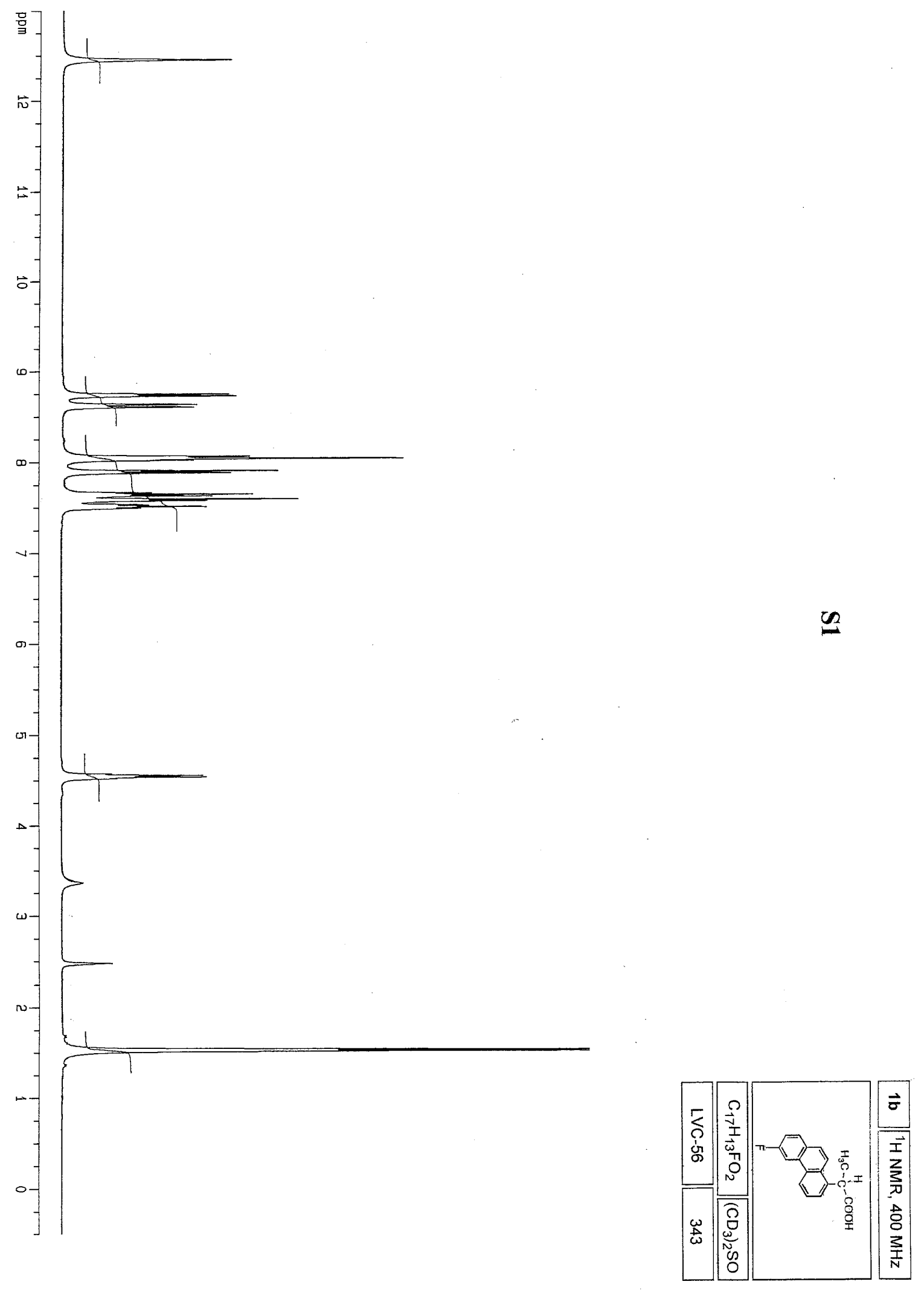

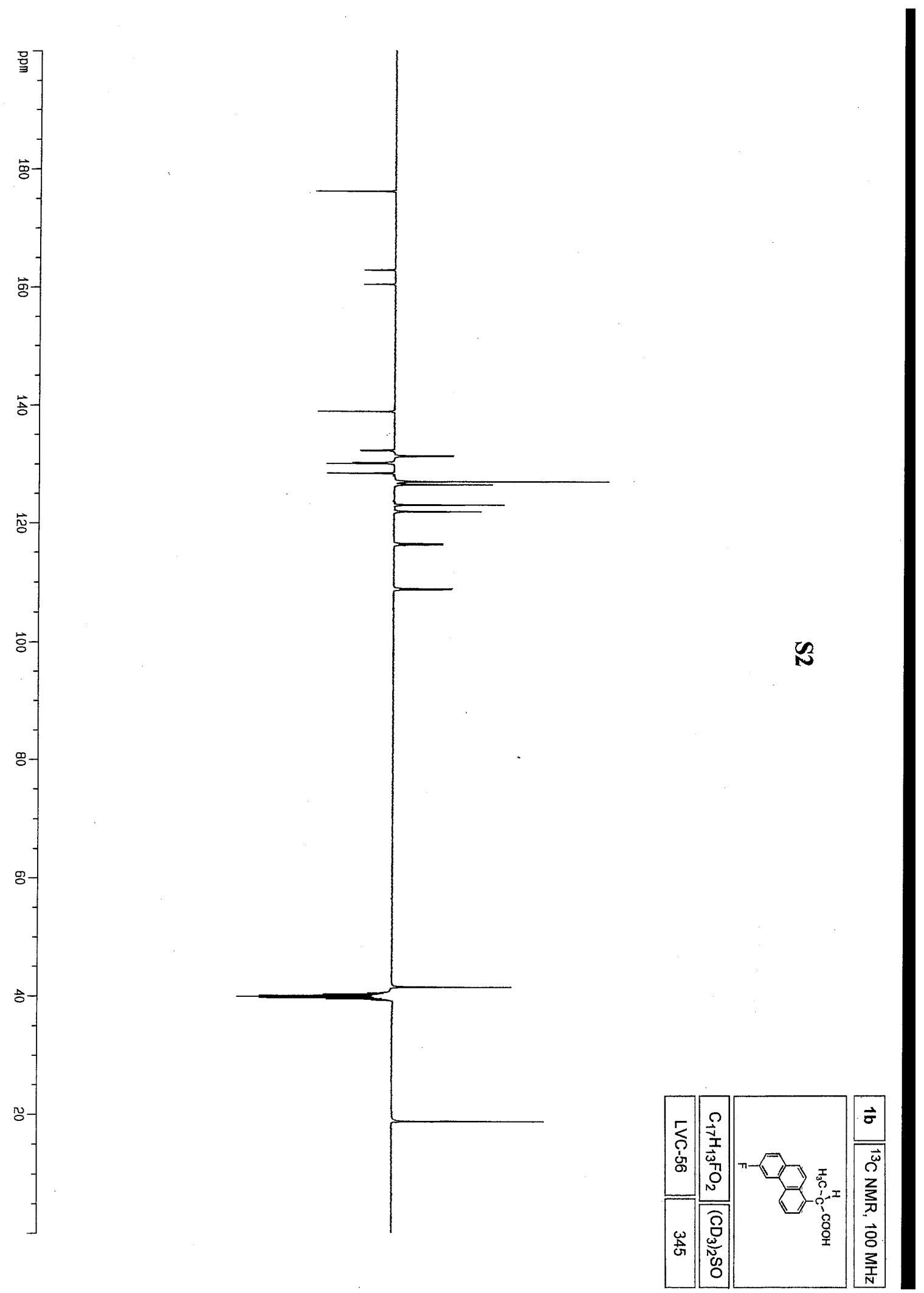


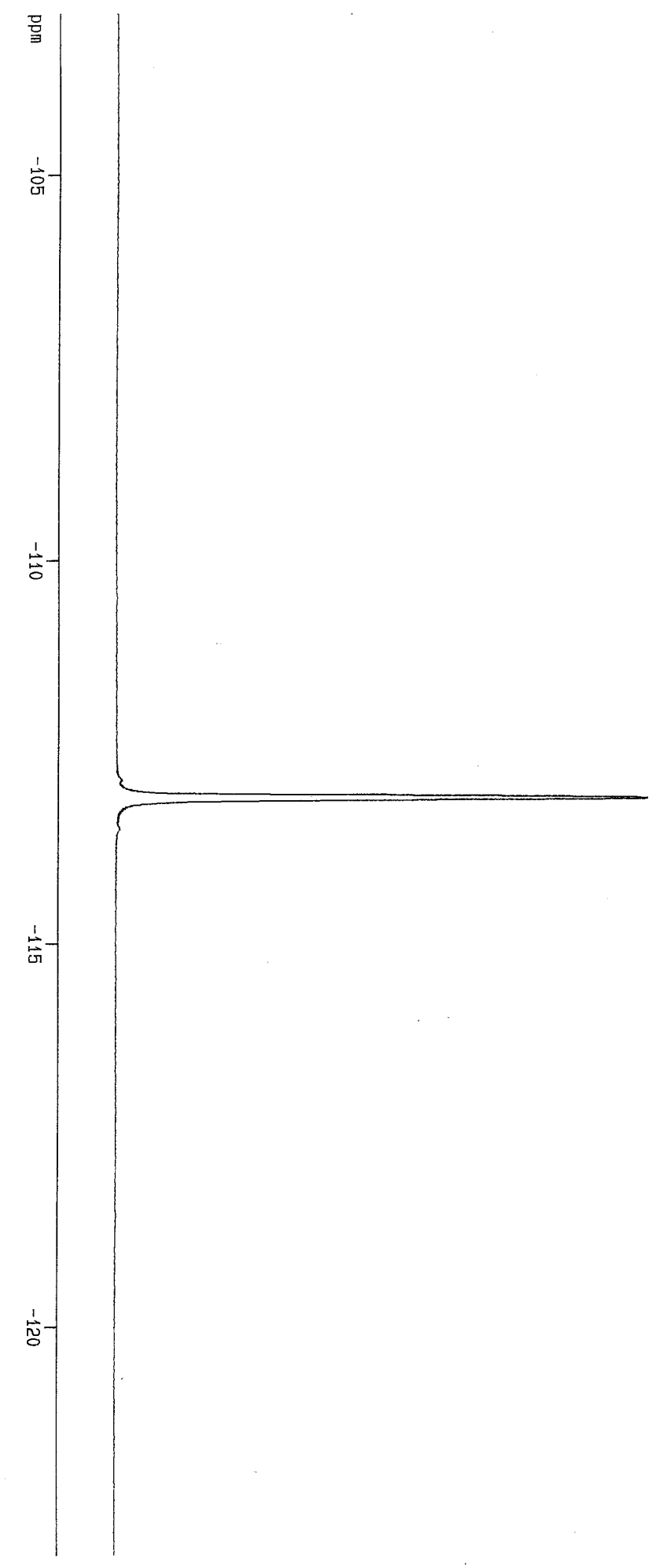

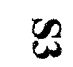

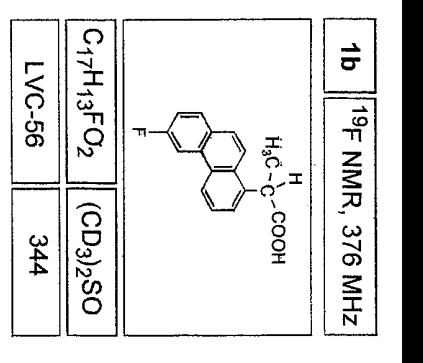




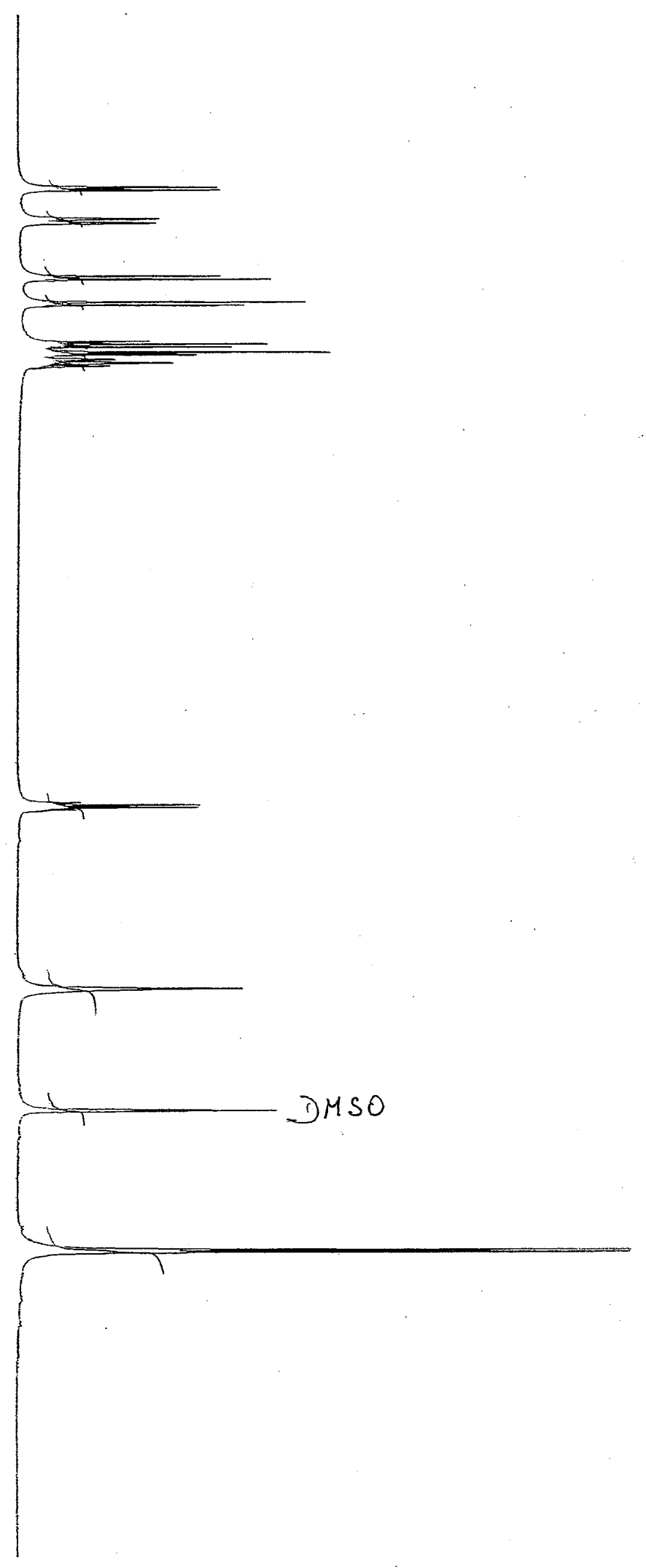

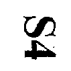

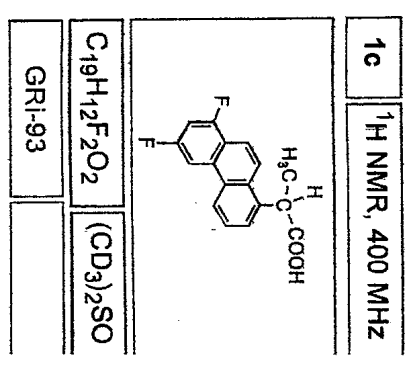



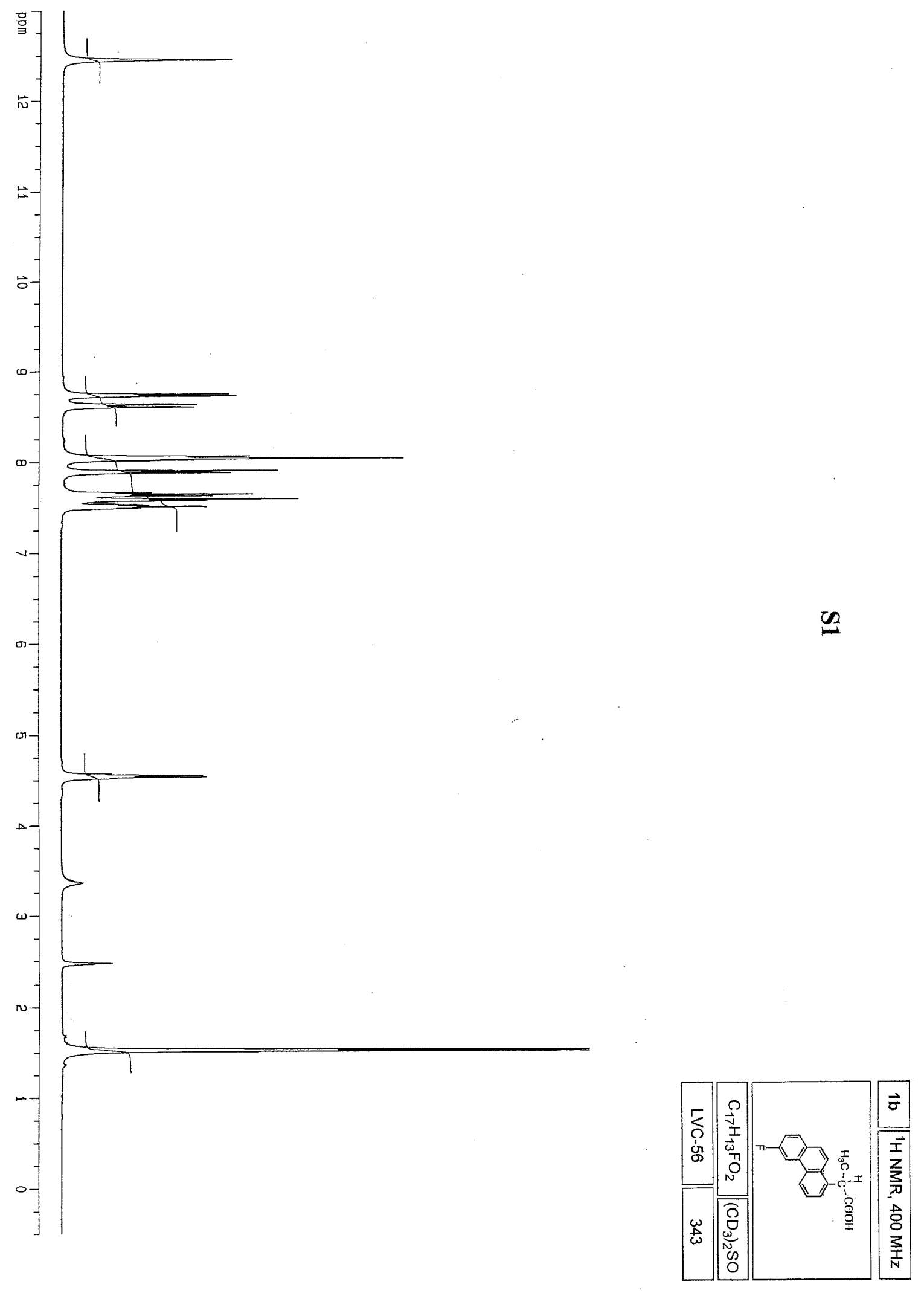


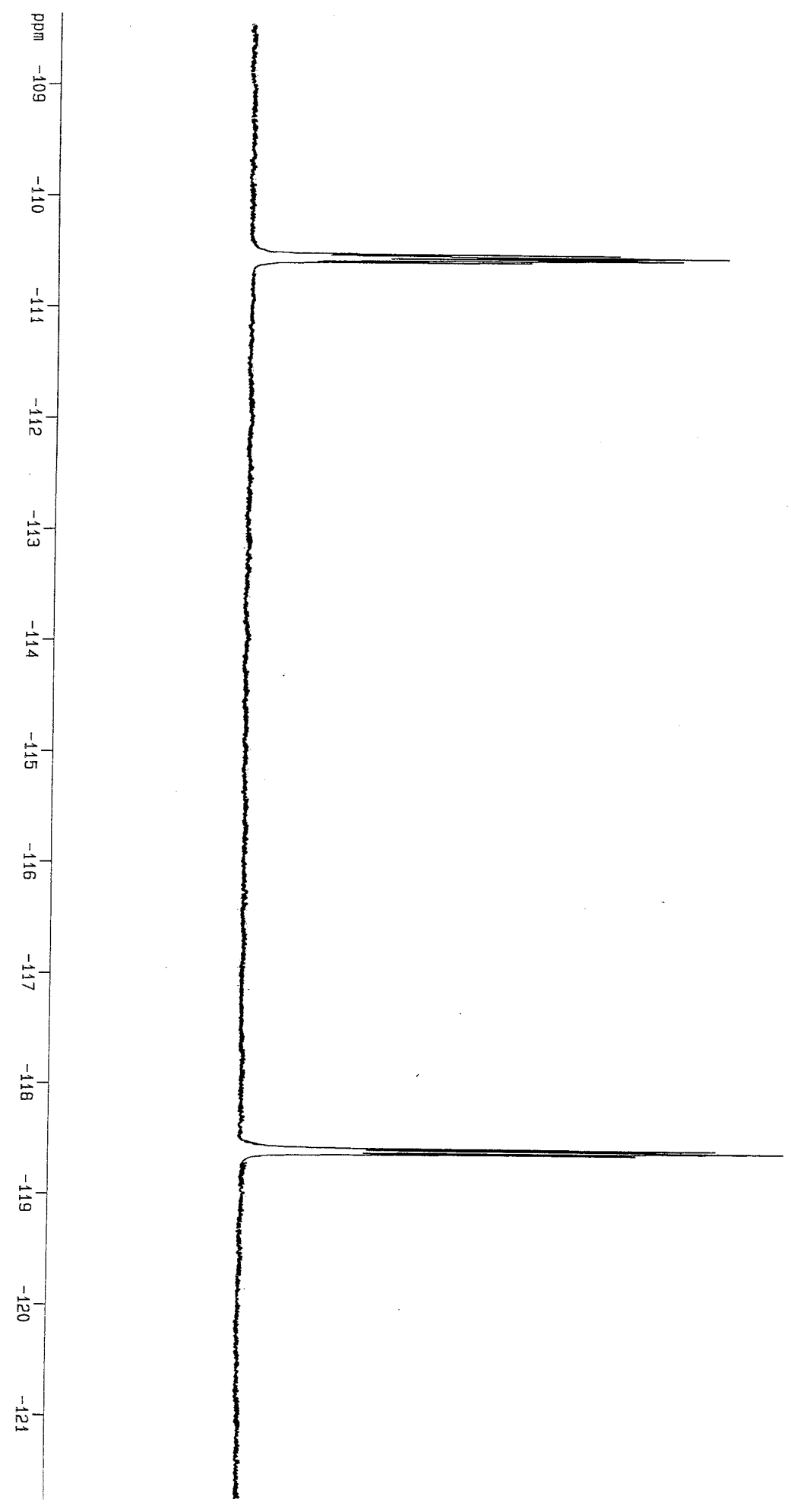

2

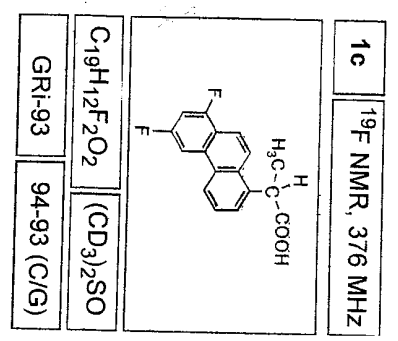




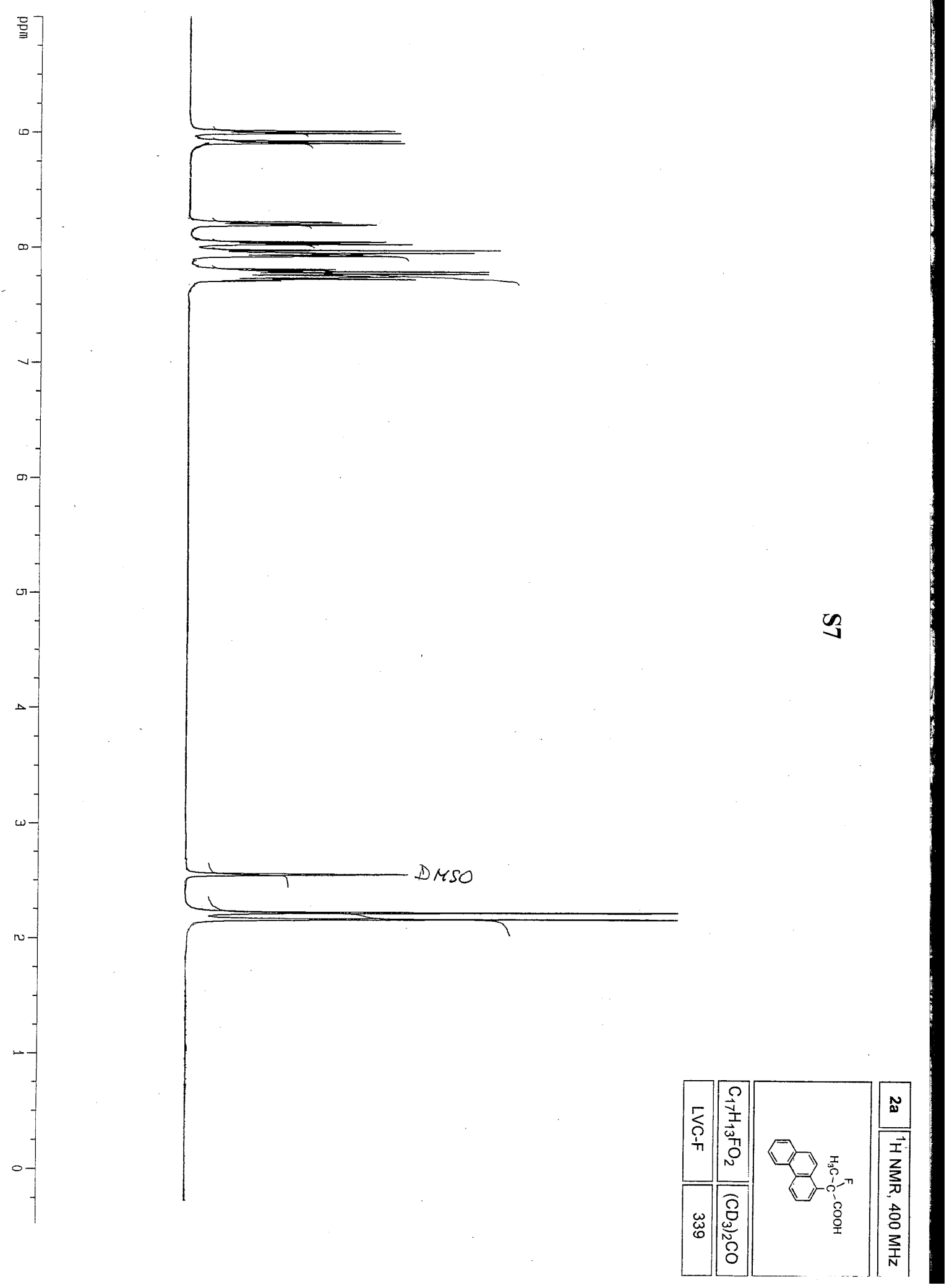




$$
f .
$$



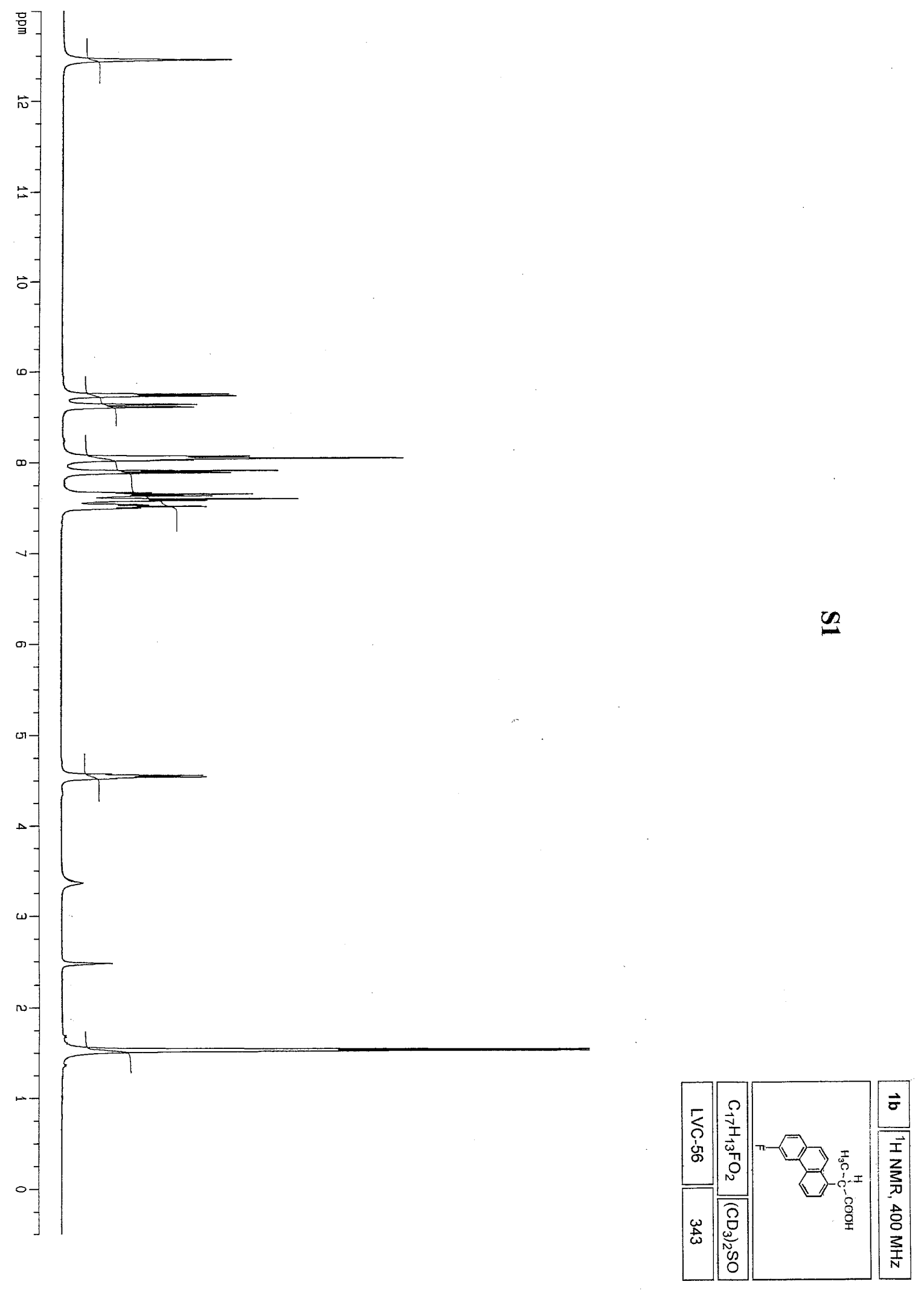


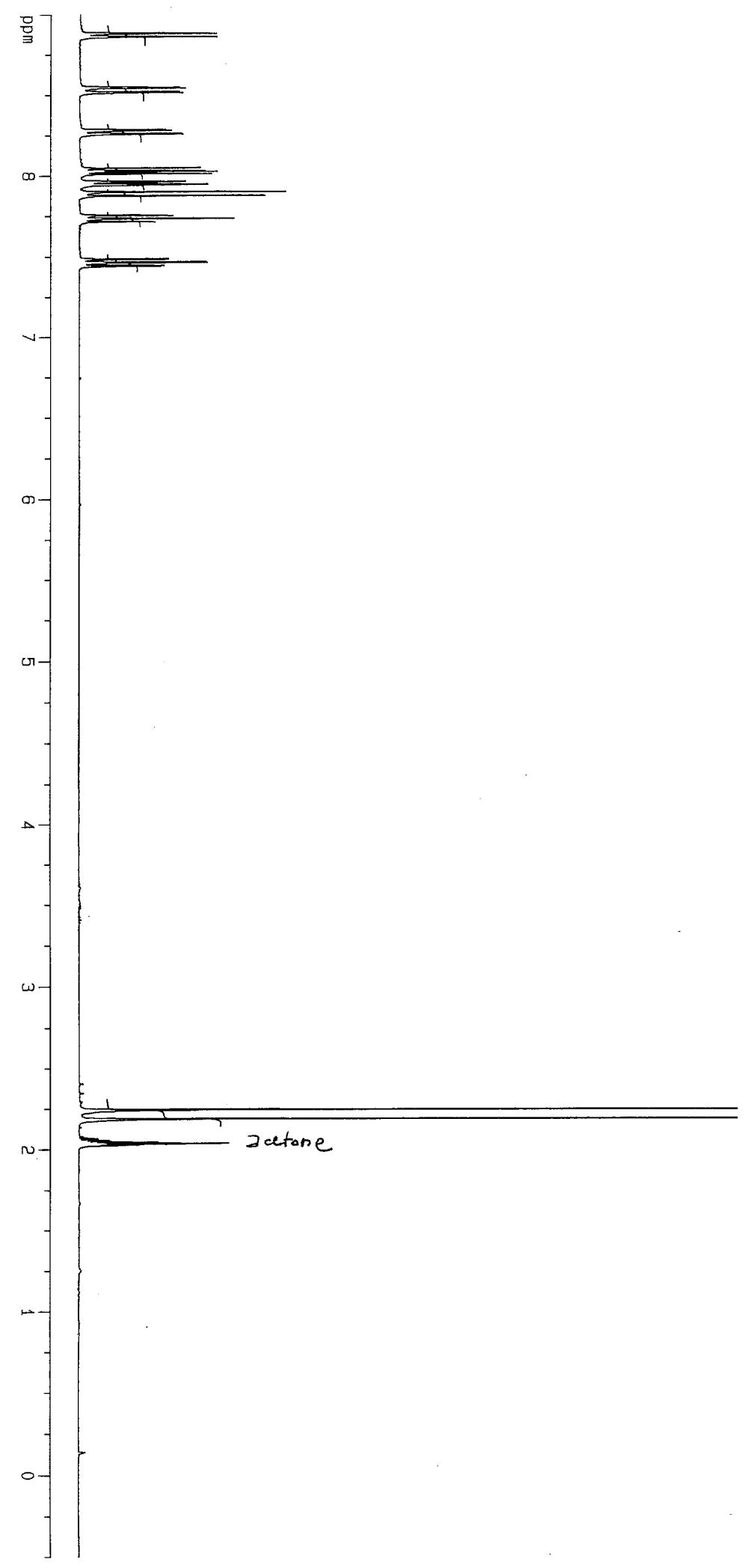

$\frac{n}{8}$

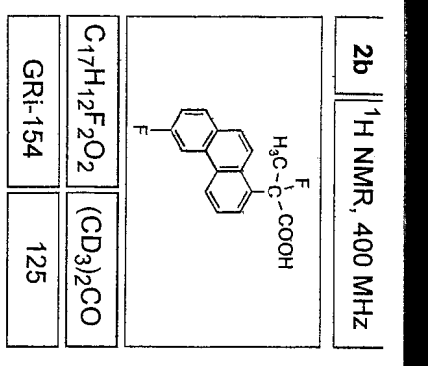




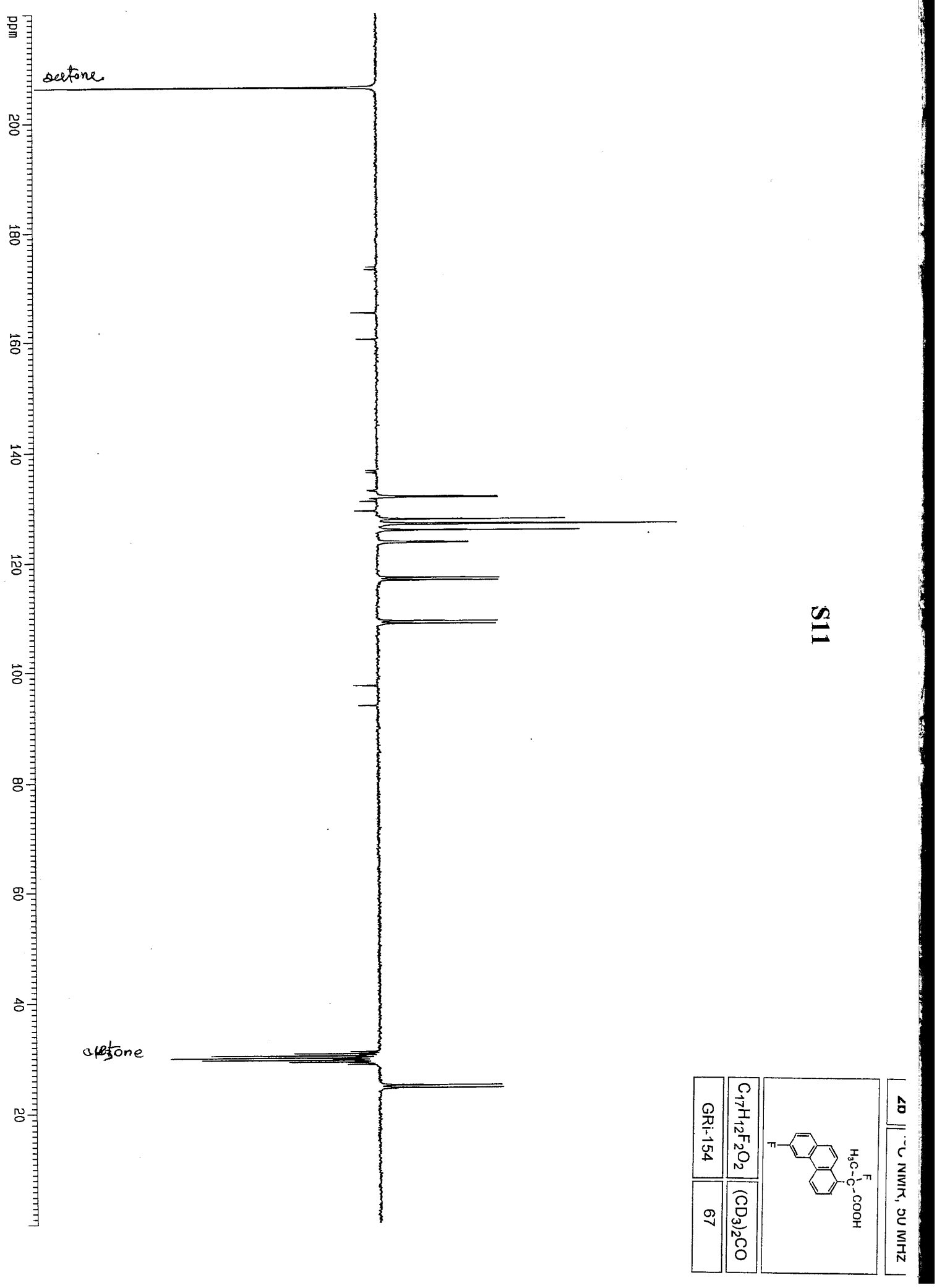




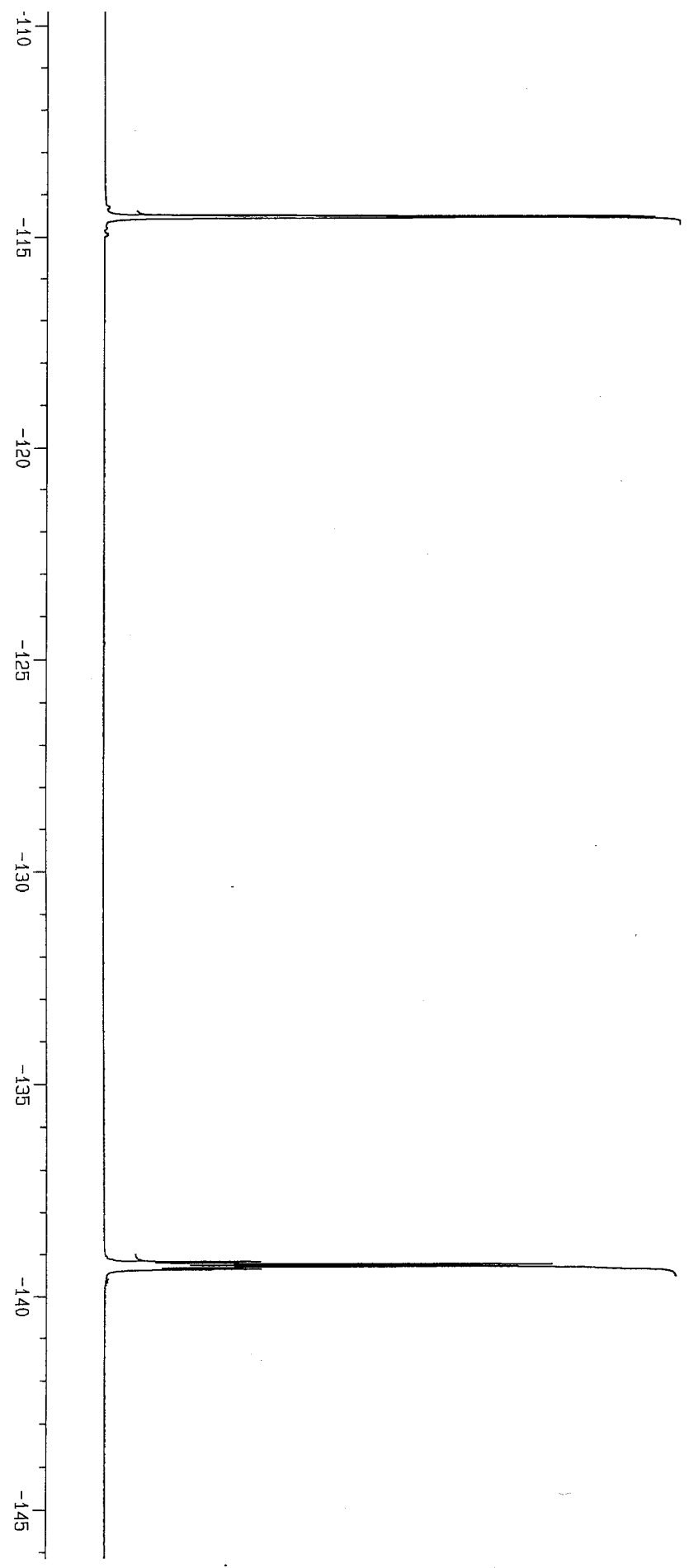

$\frac{N}{N}$

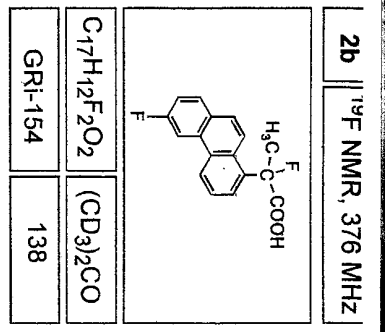




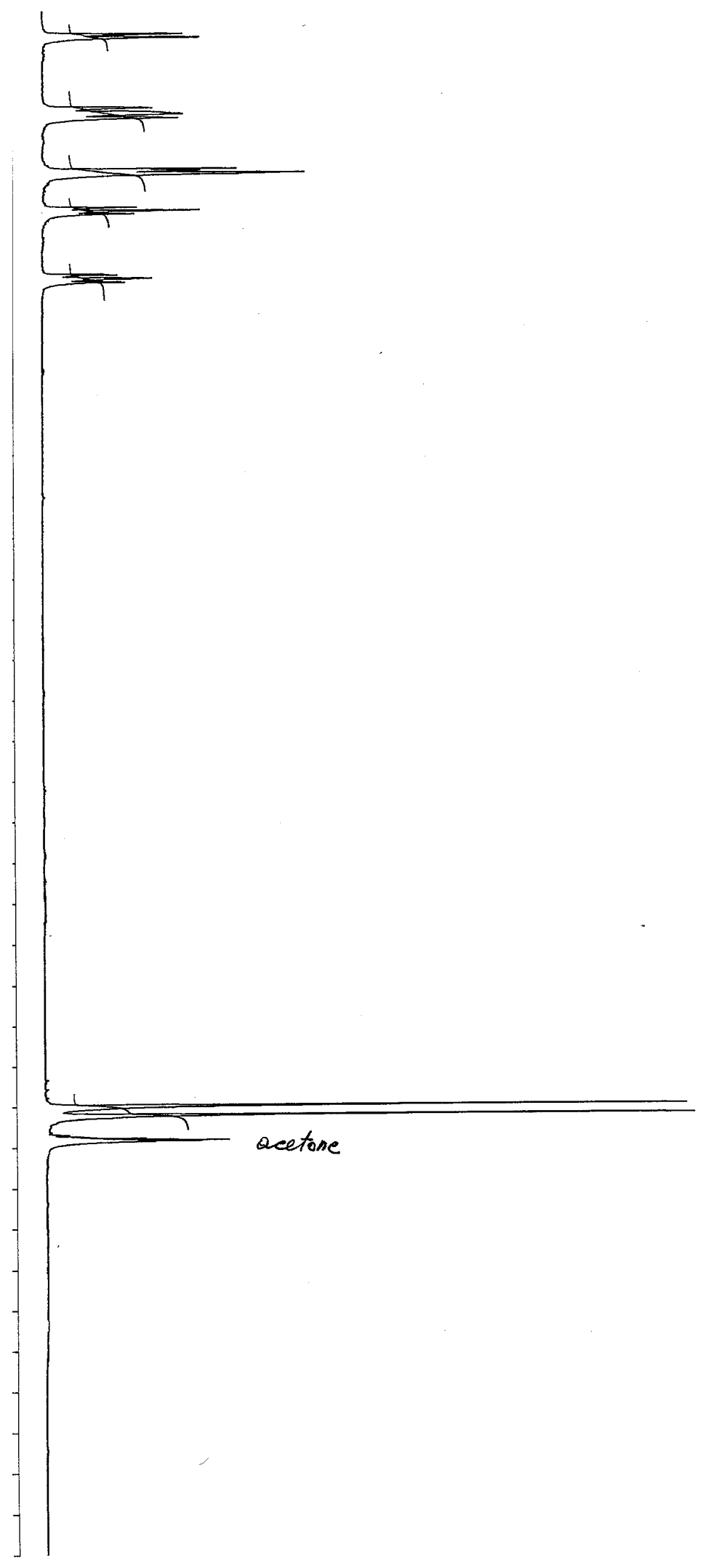

$\underline{w}$

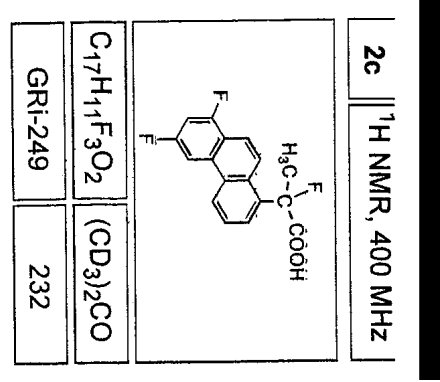



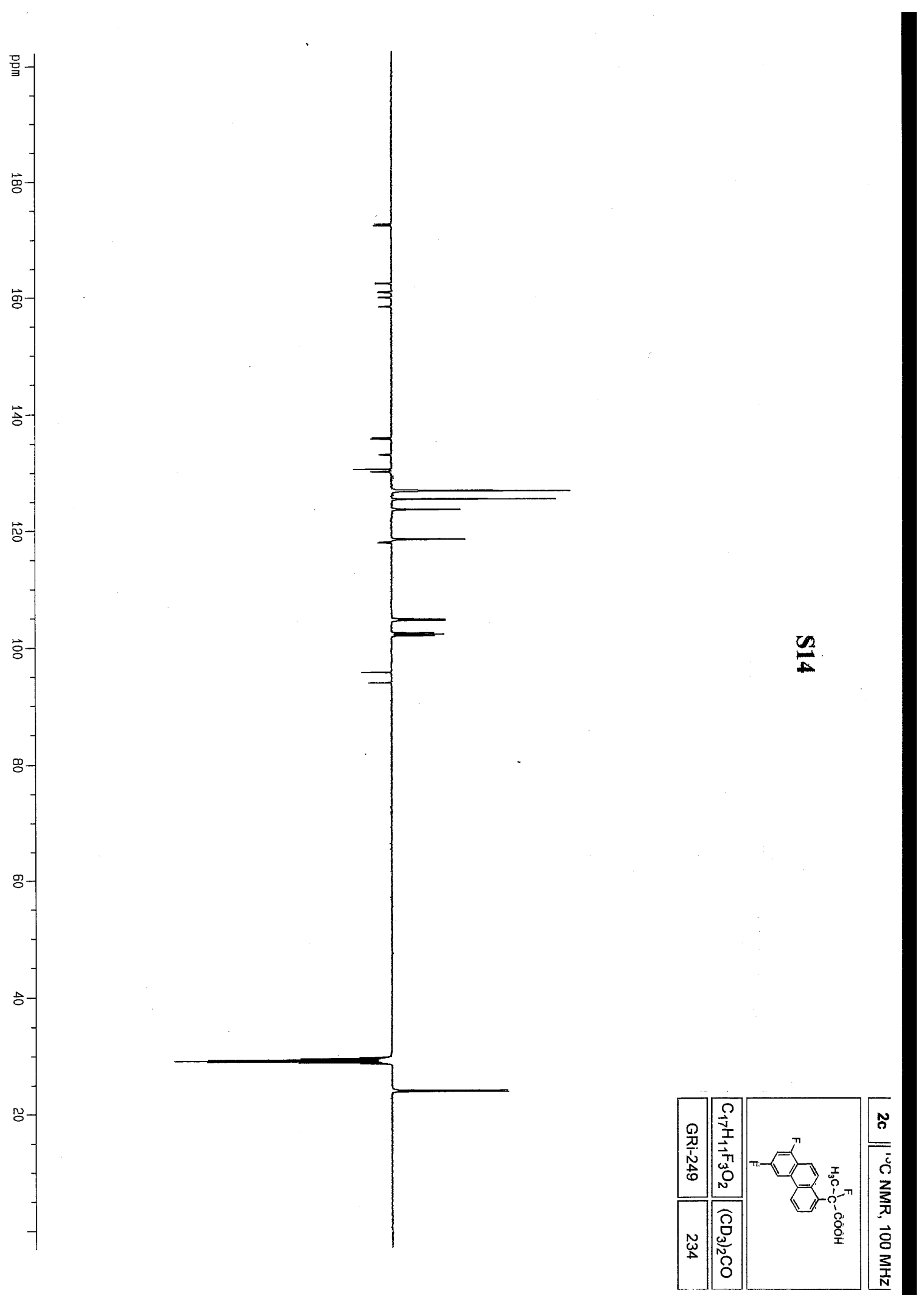

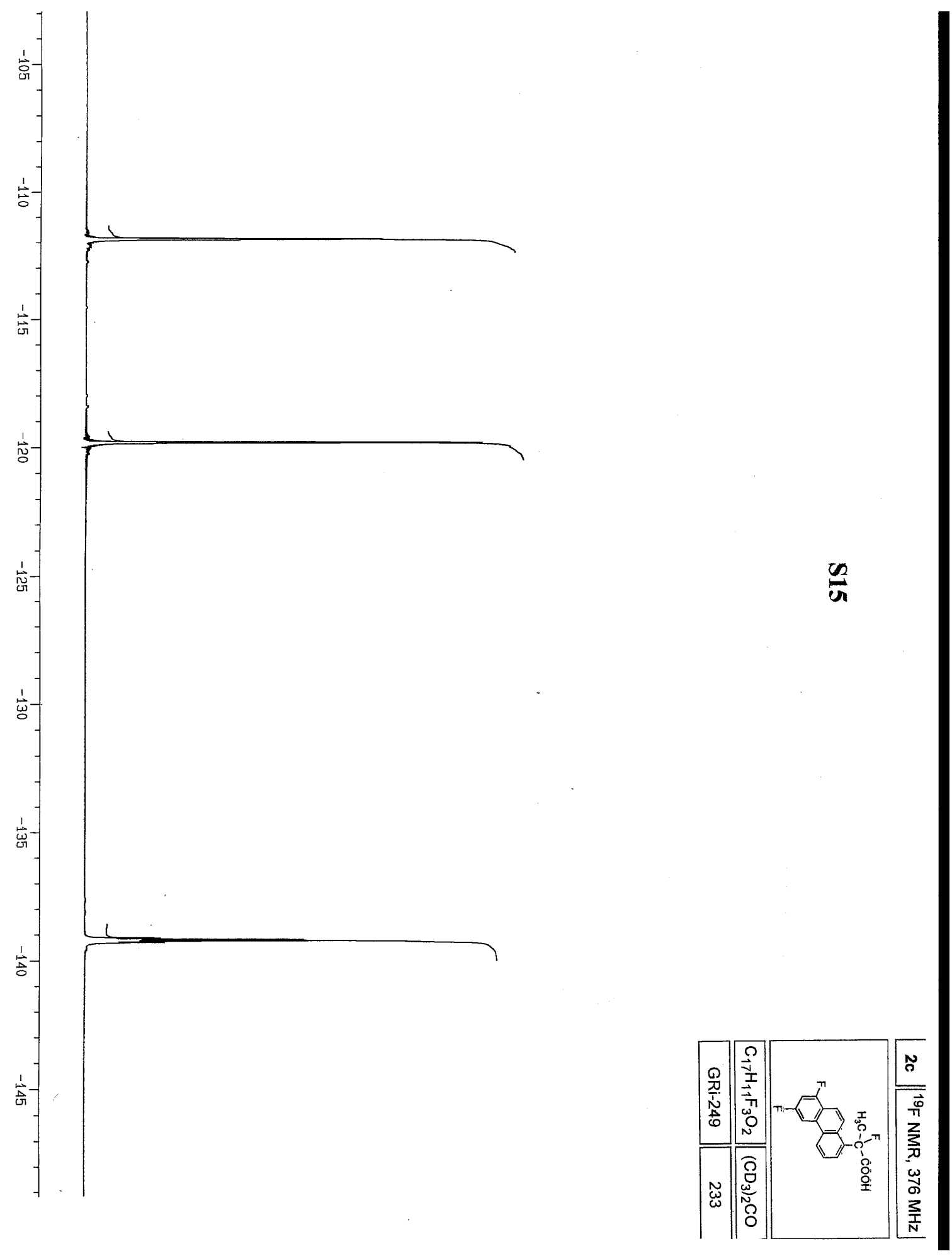


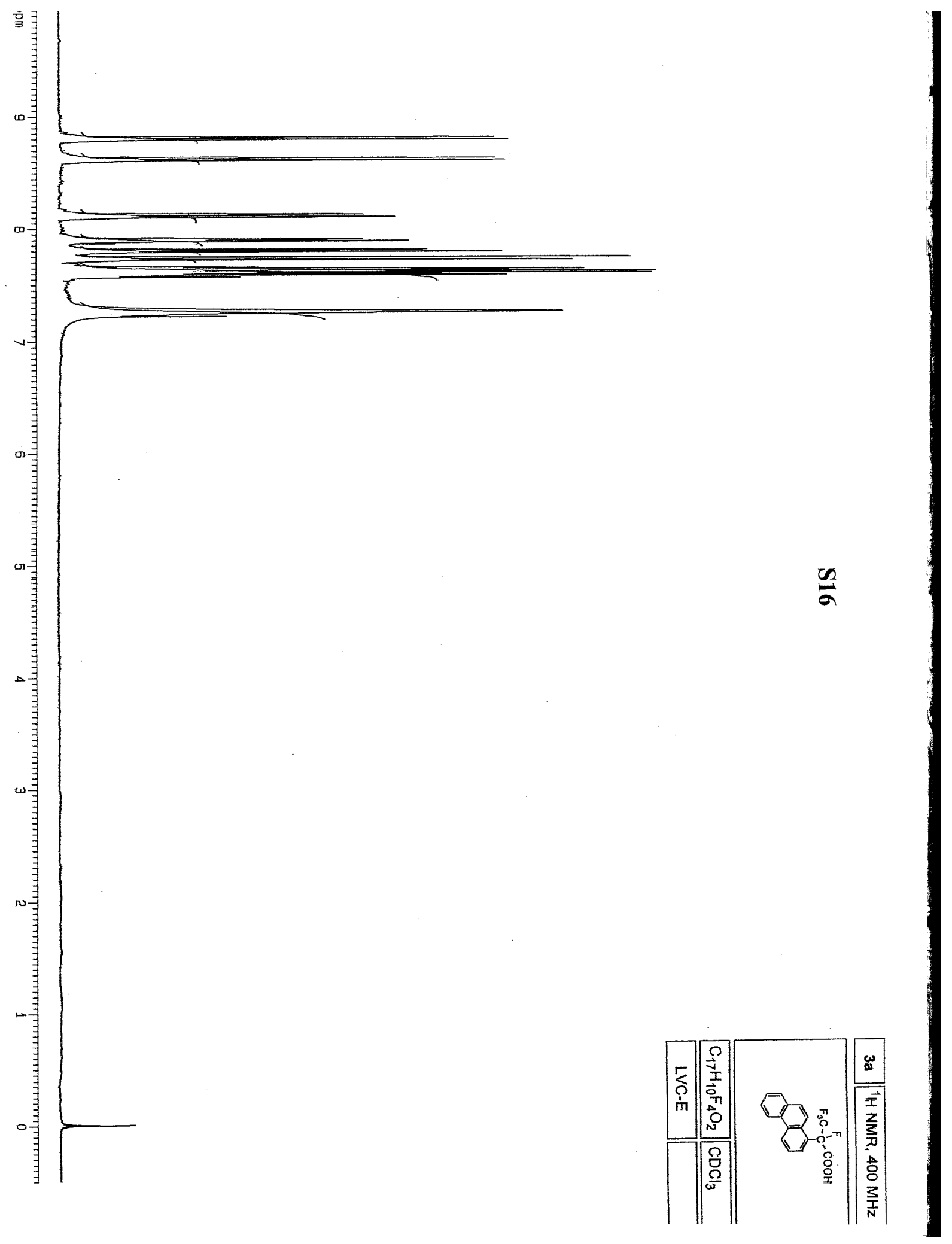




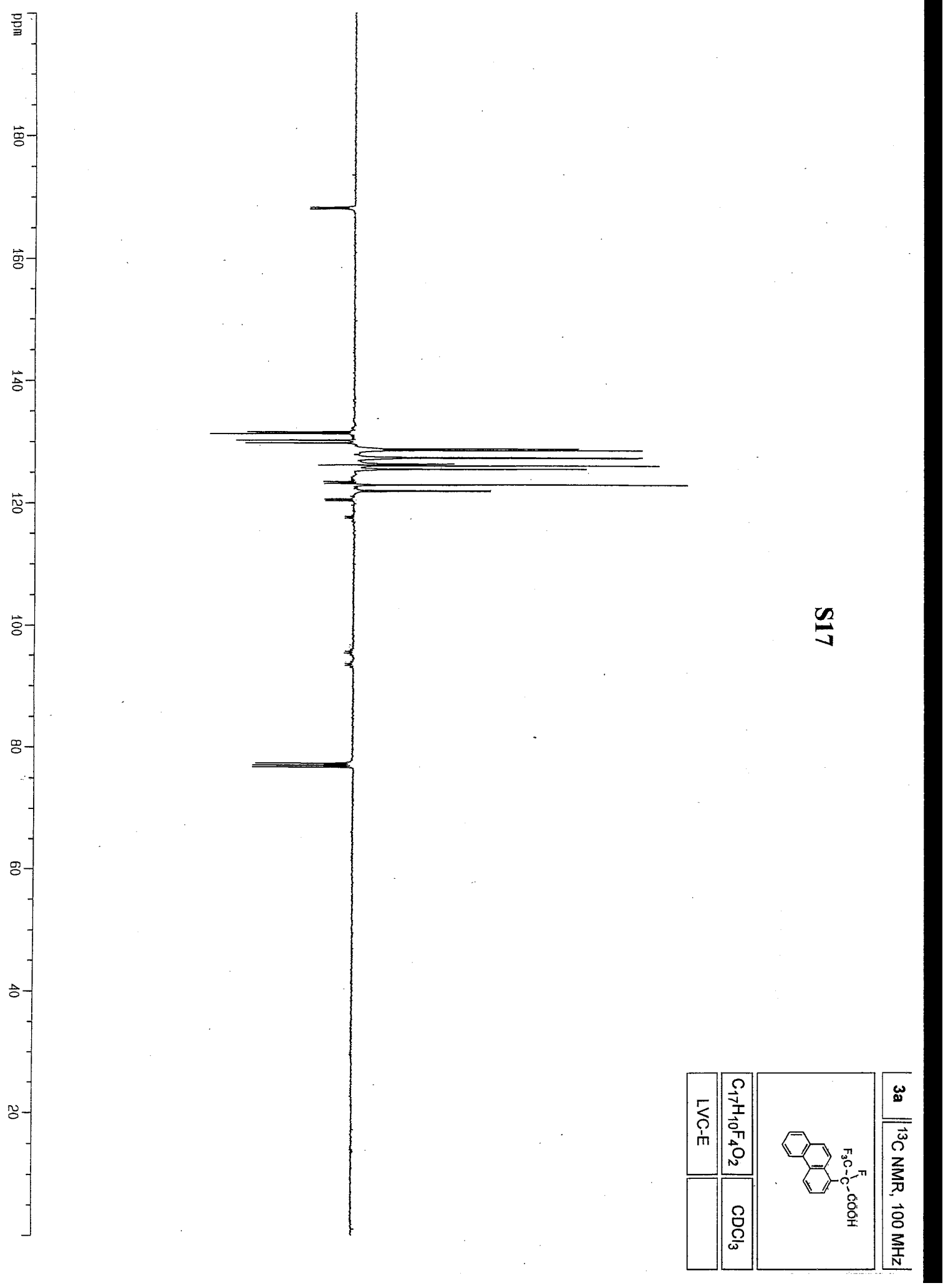



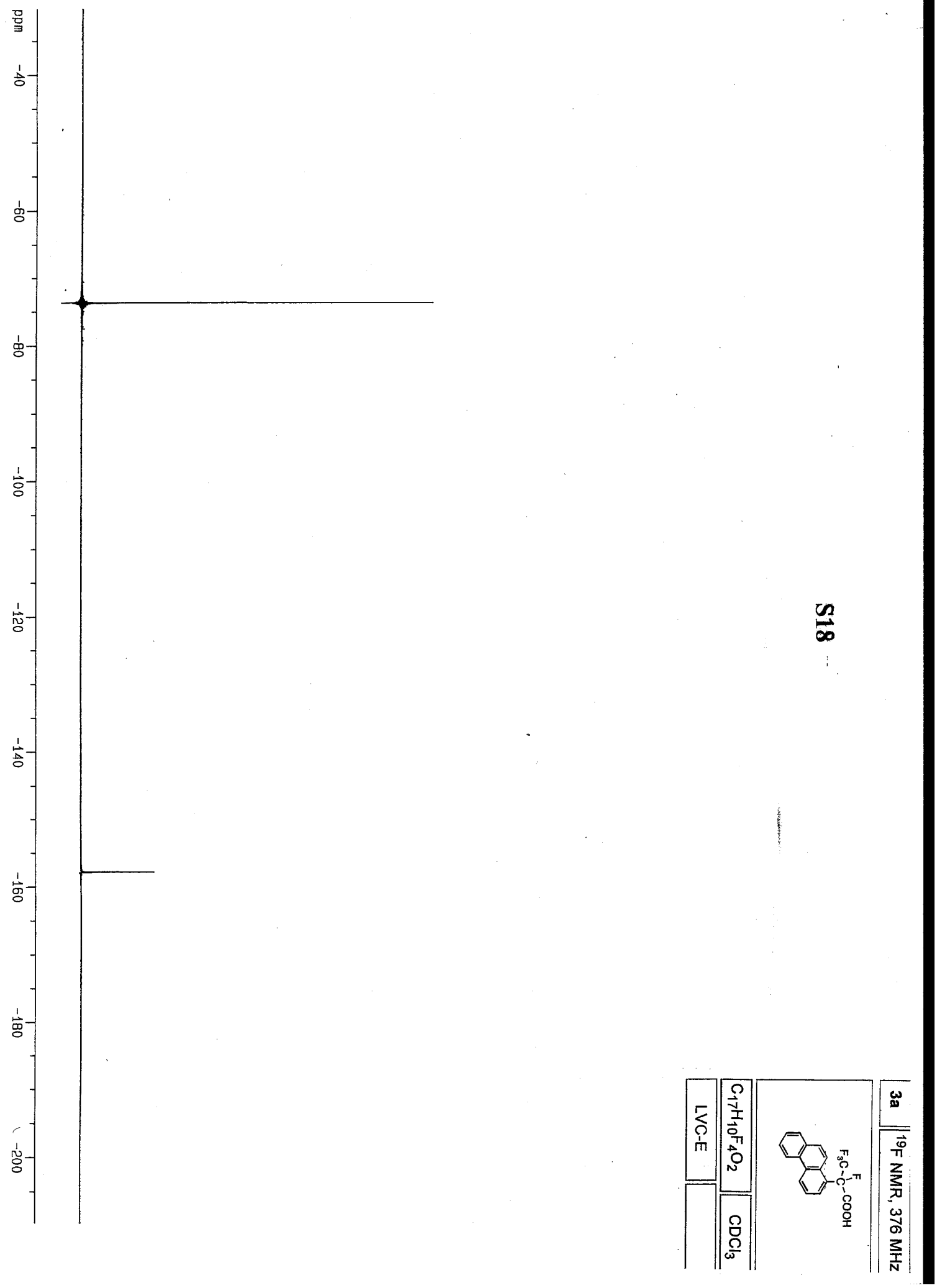


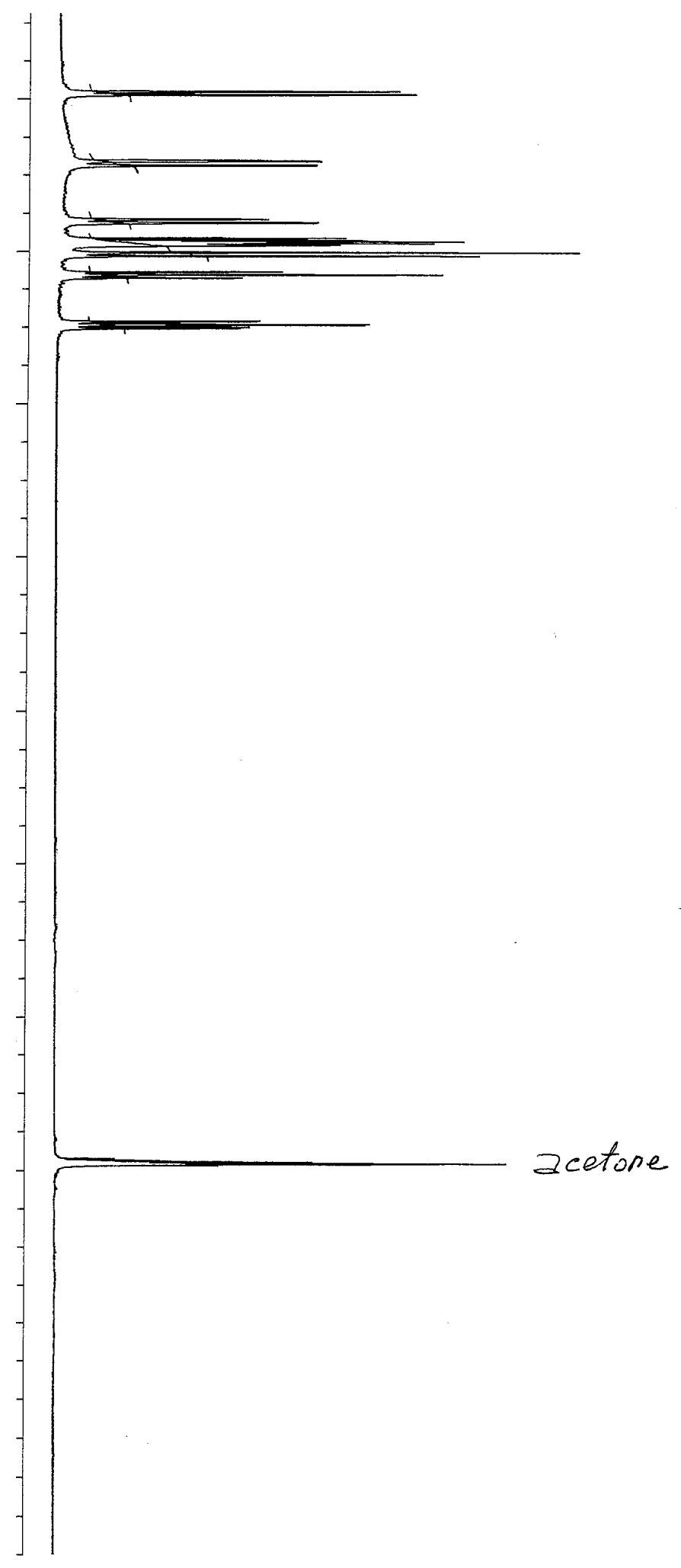

$\frac{\infty}{6}$

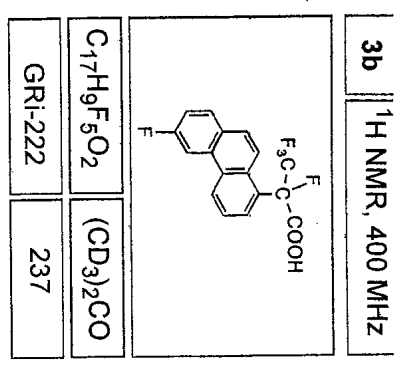



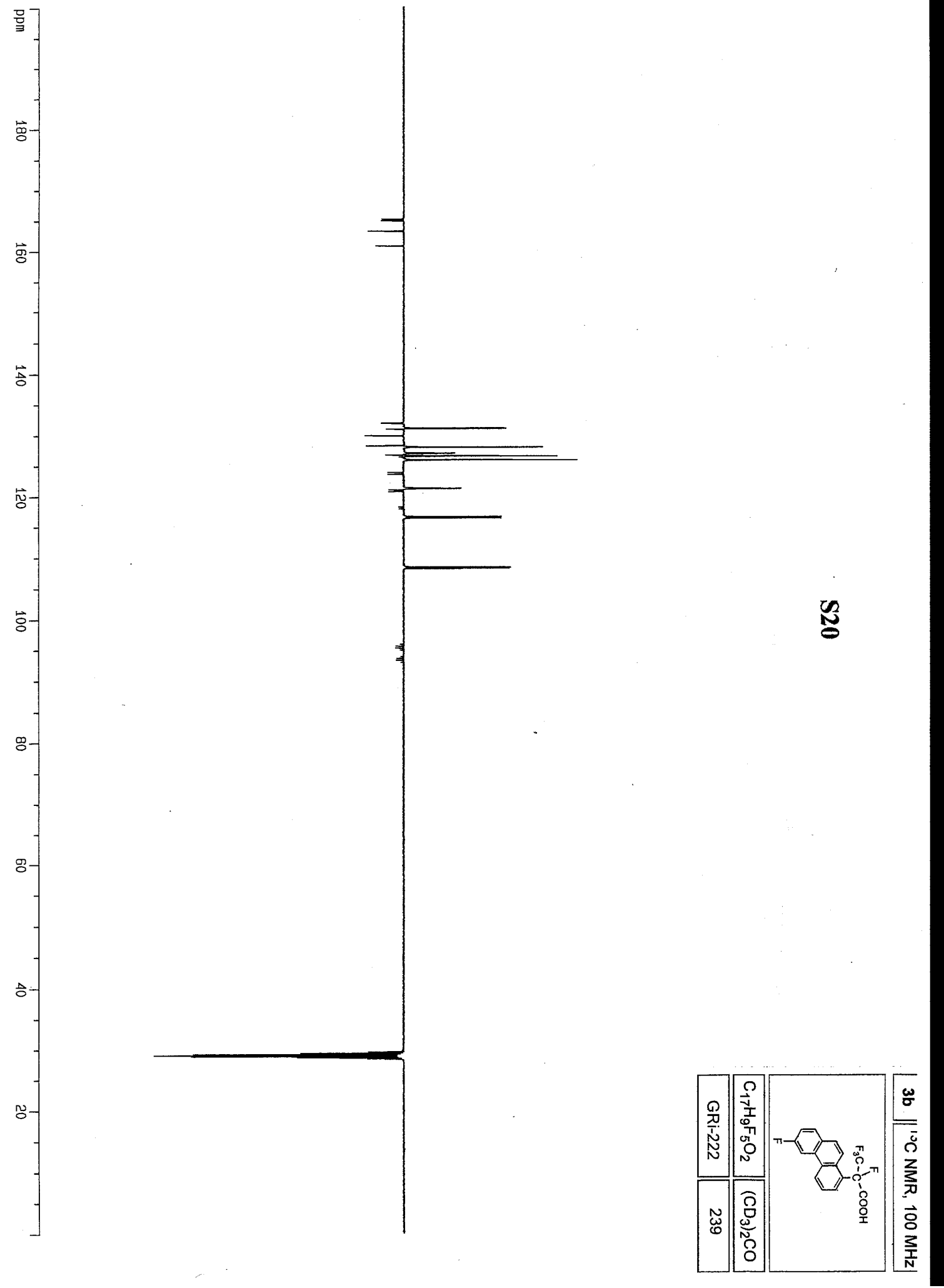

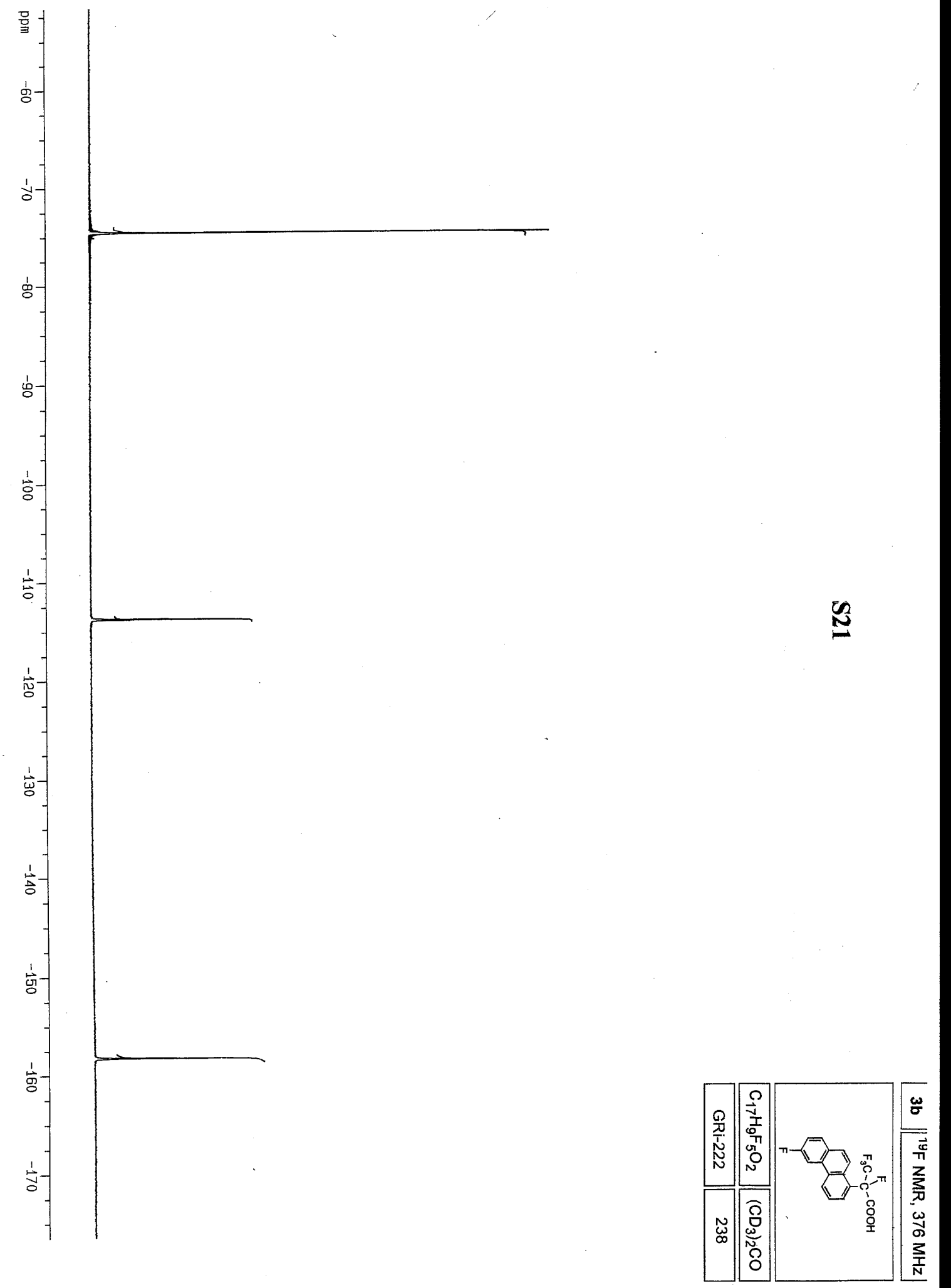


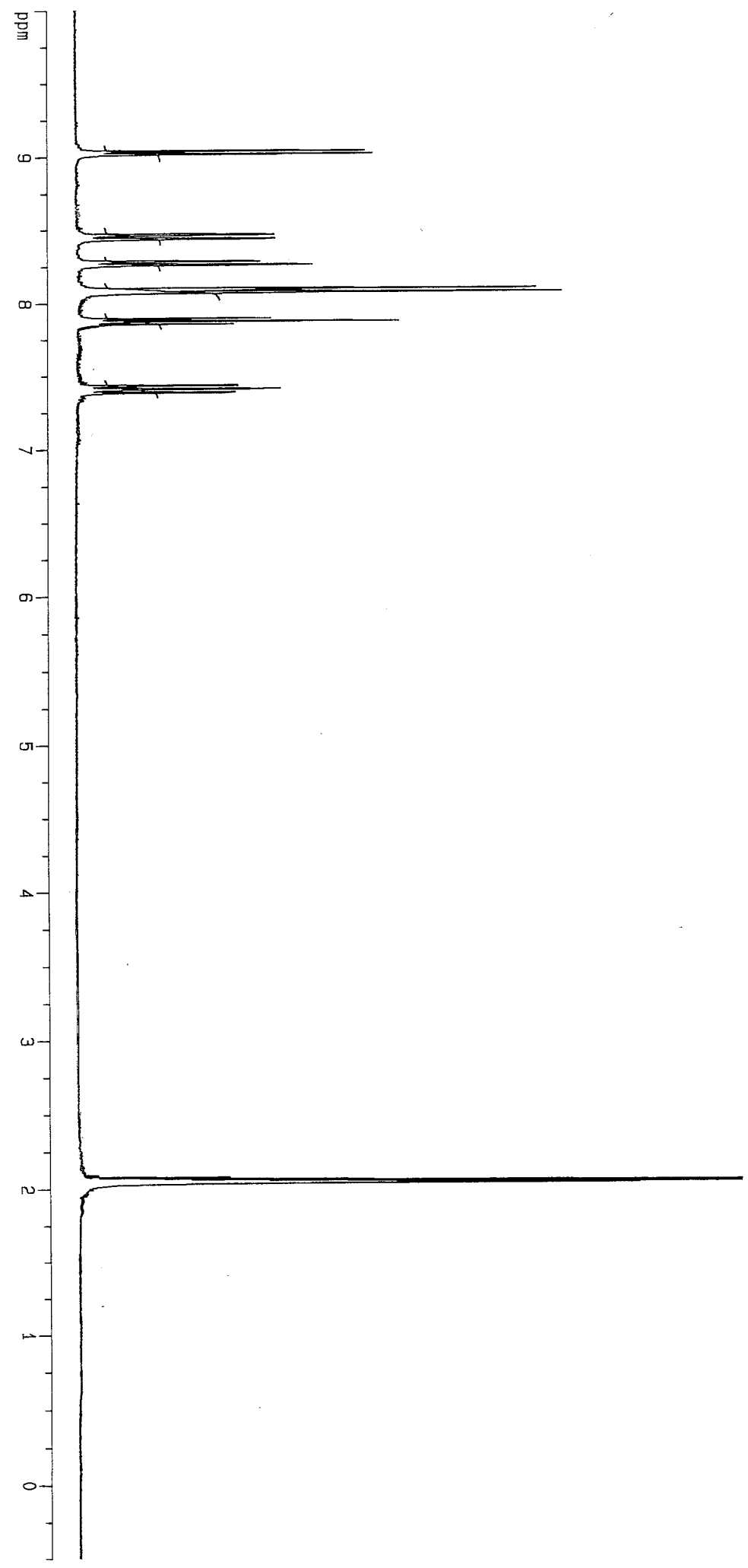

$\mathbb{N}$

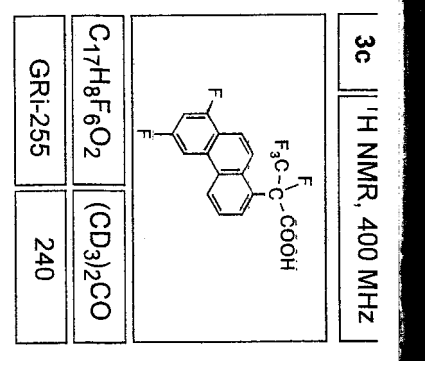




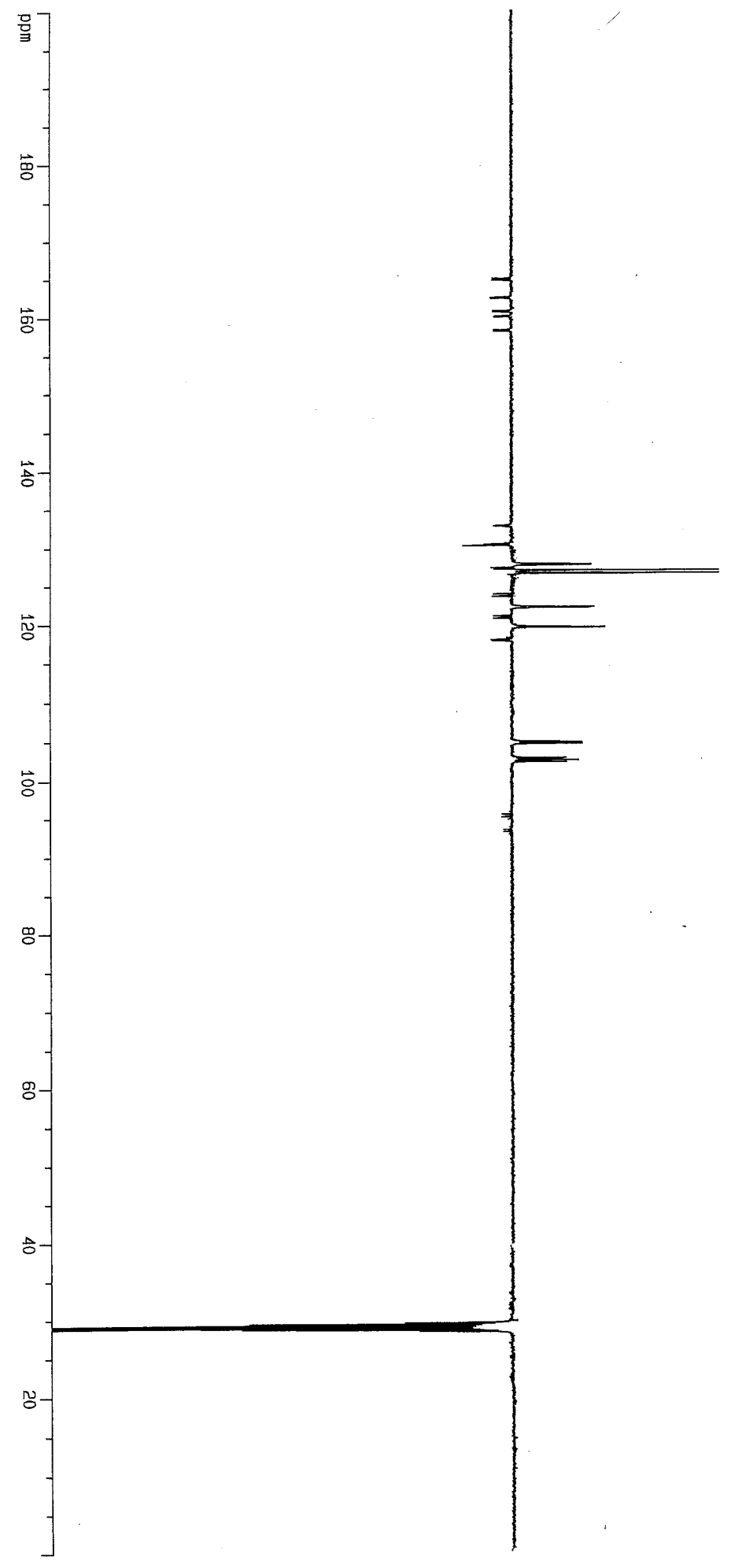

\&

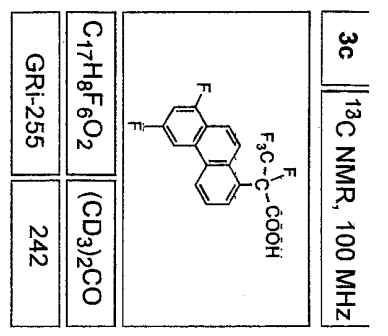




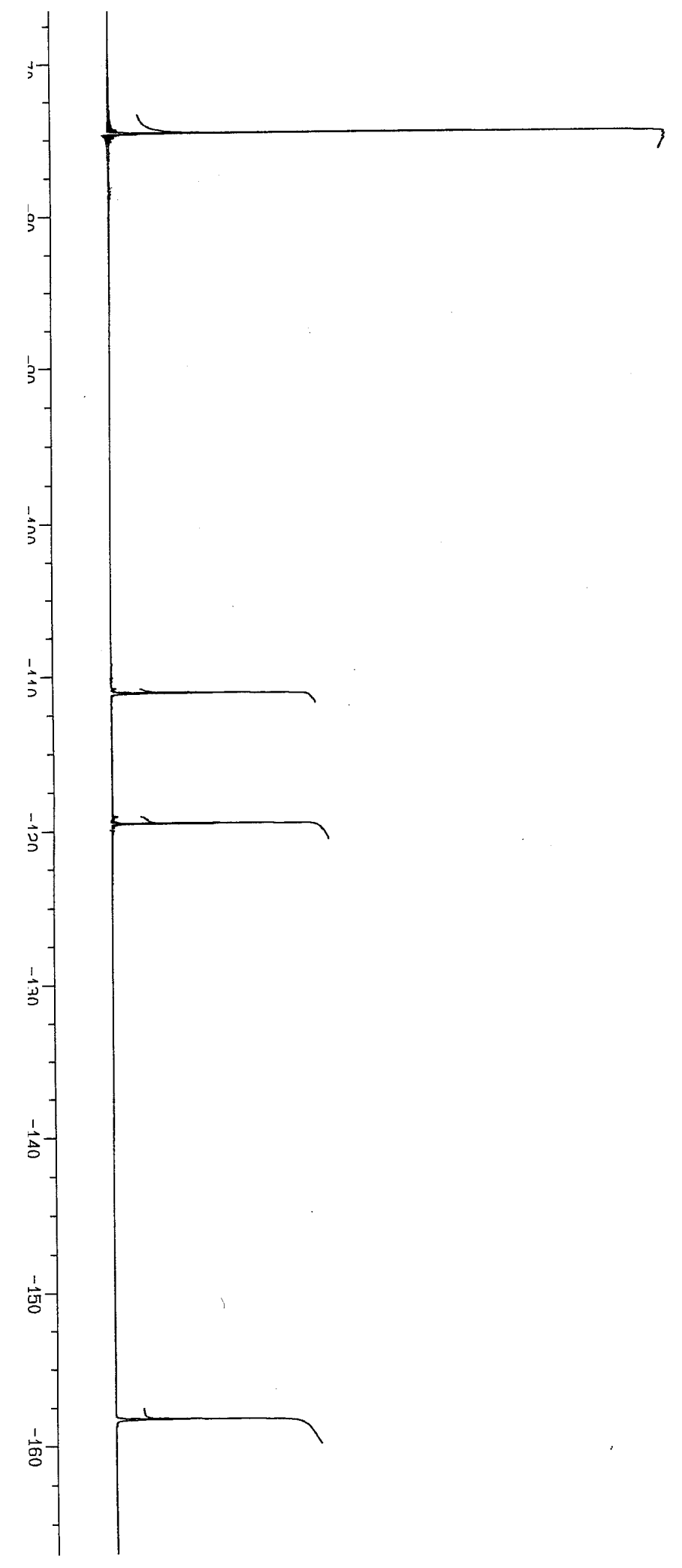

5

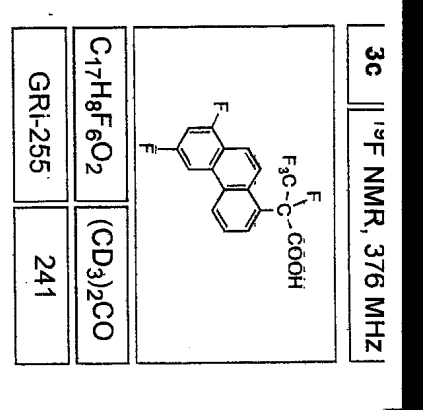




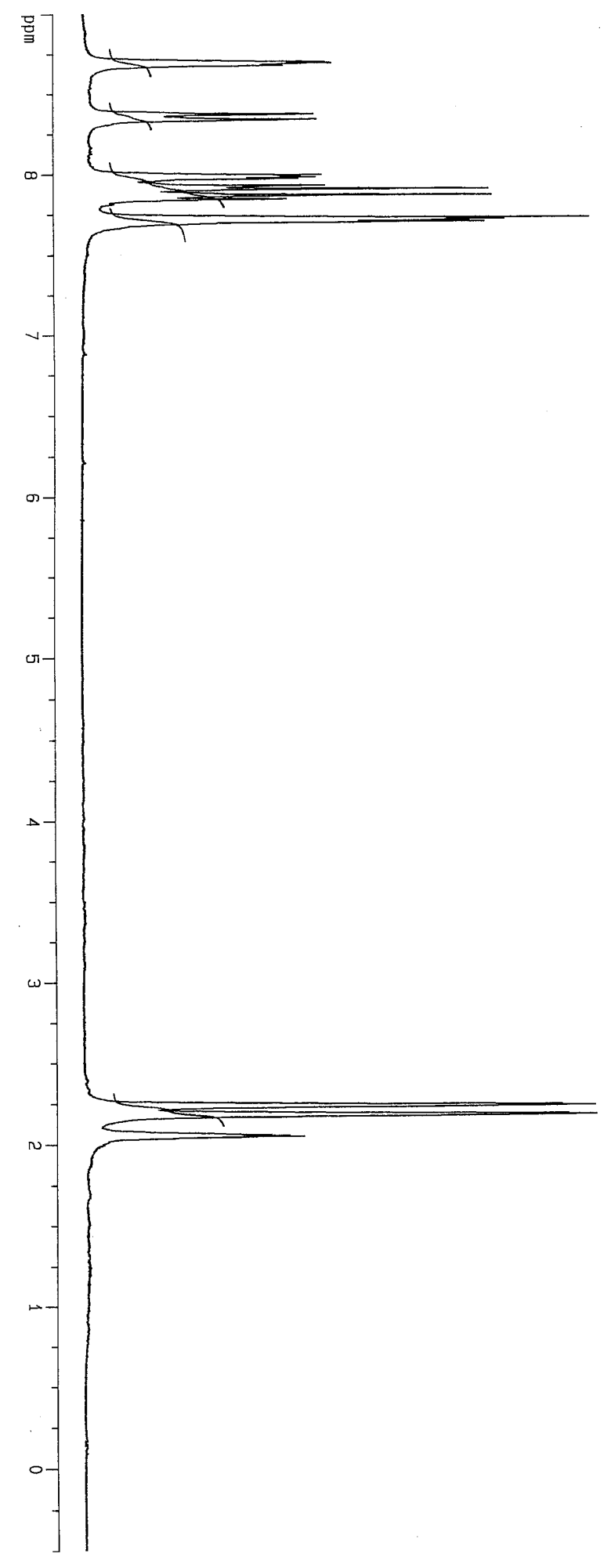

s

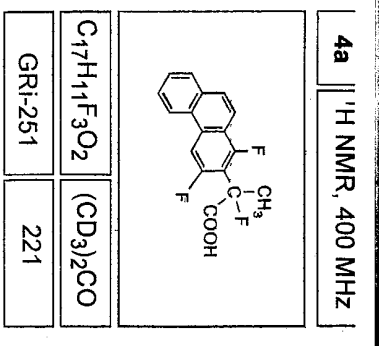




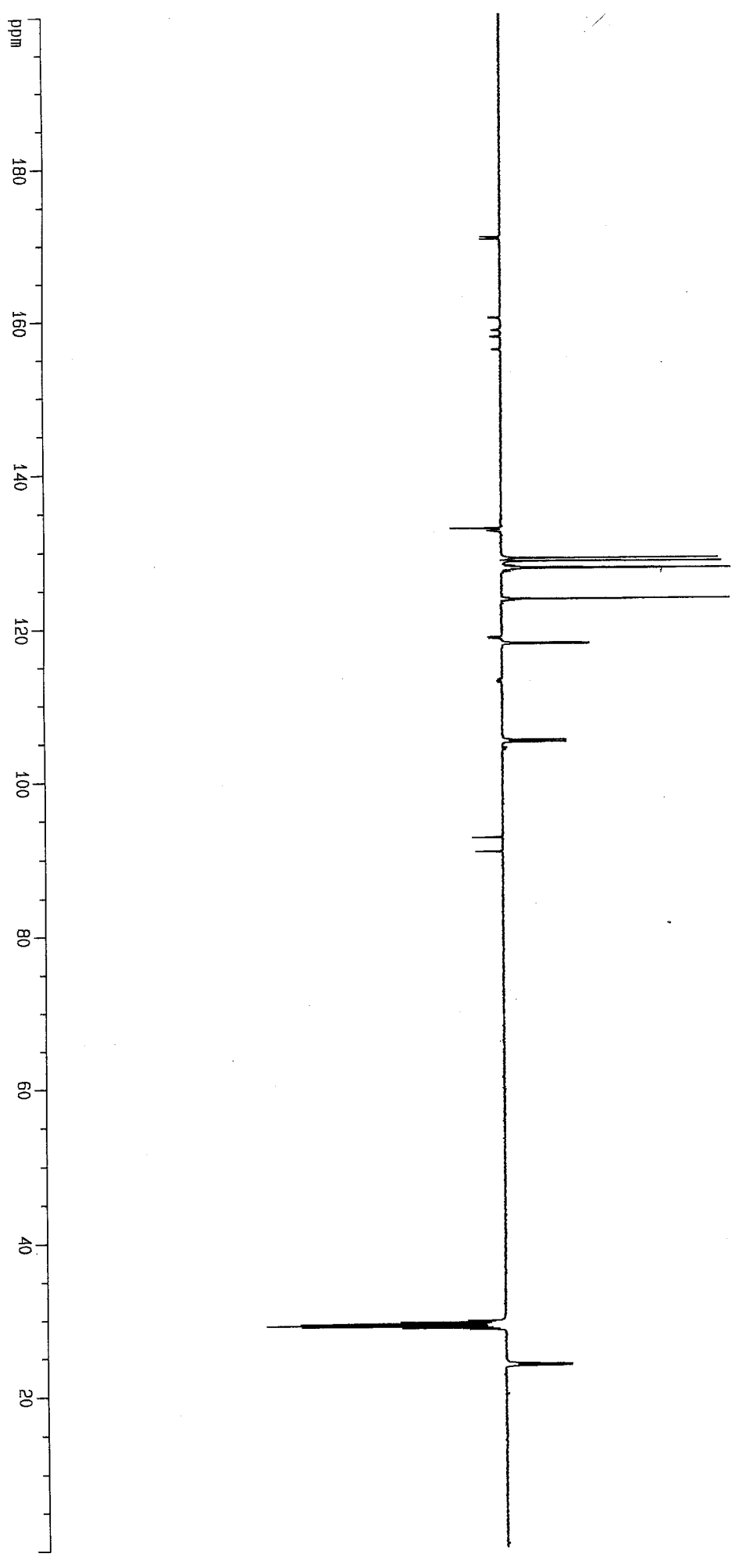

సू

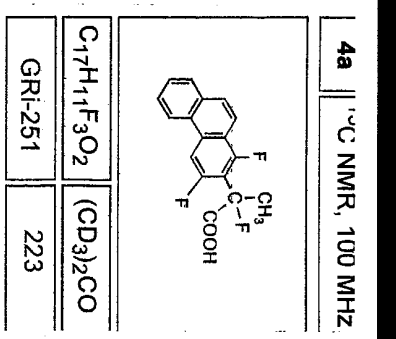



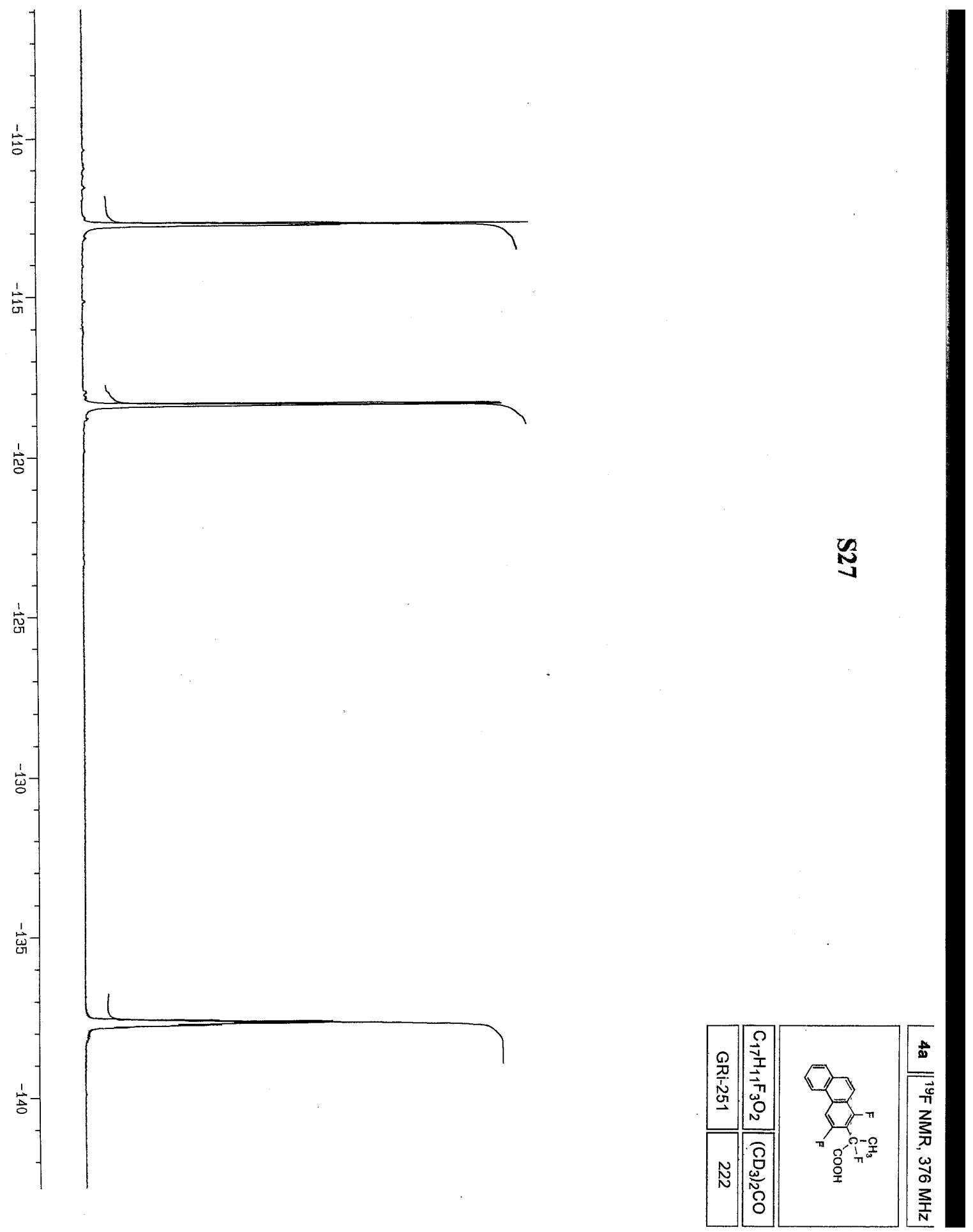


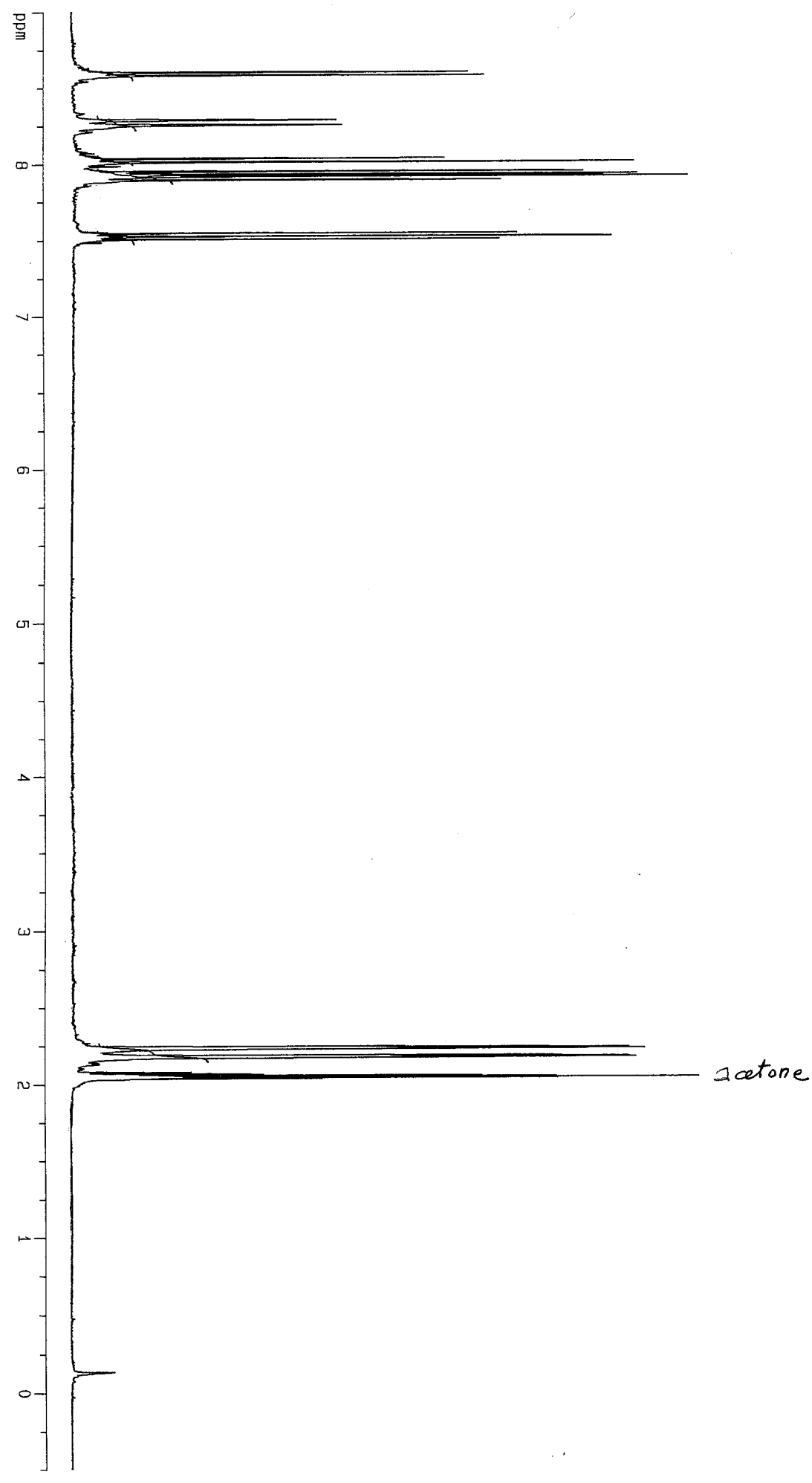

$\stackrel{\infty}{N}$

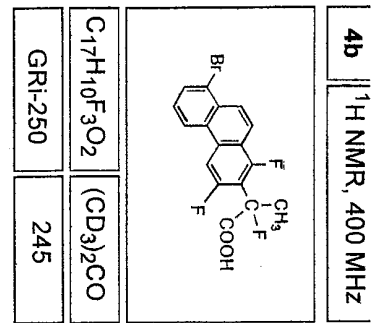



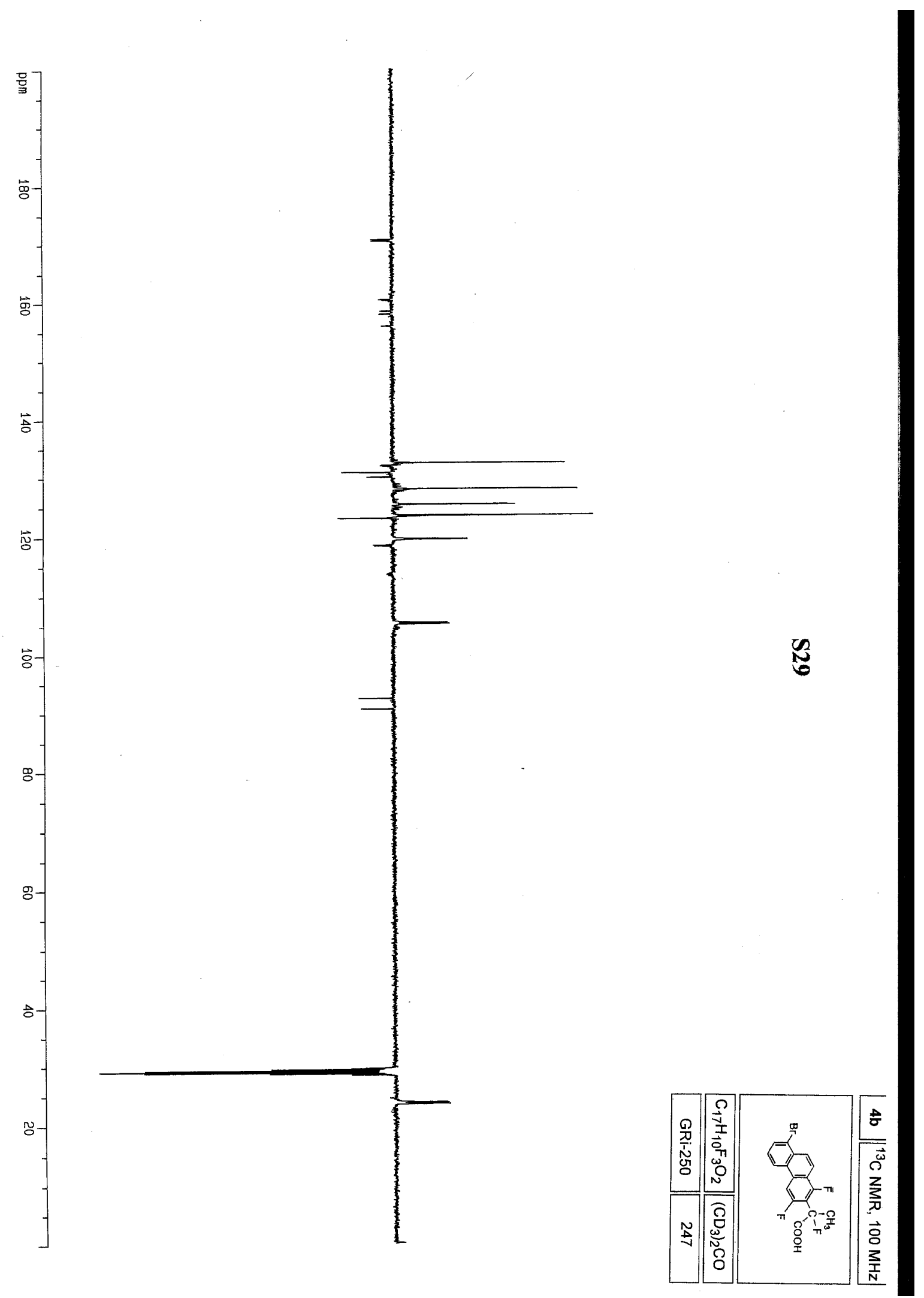


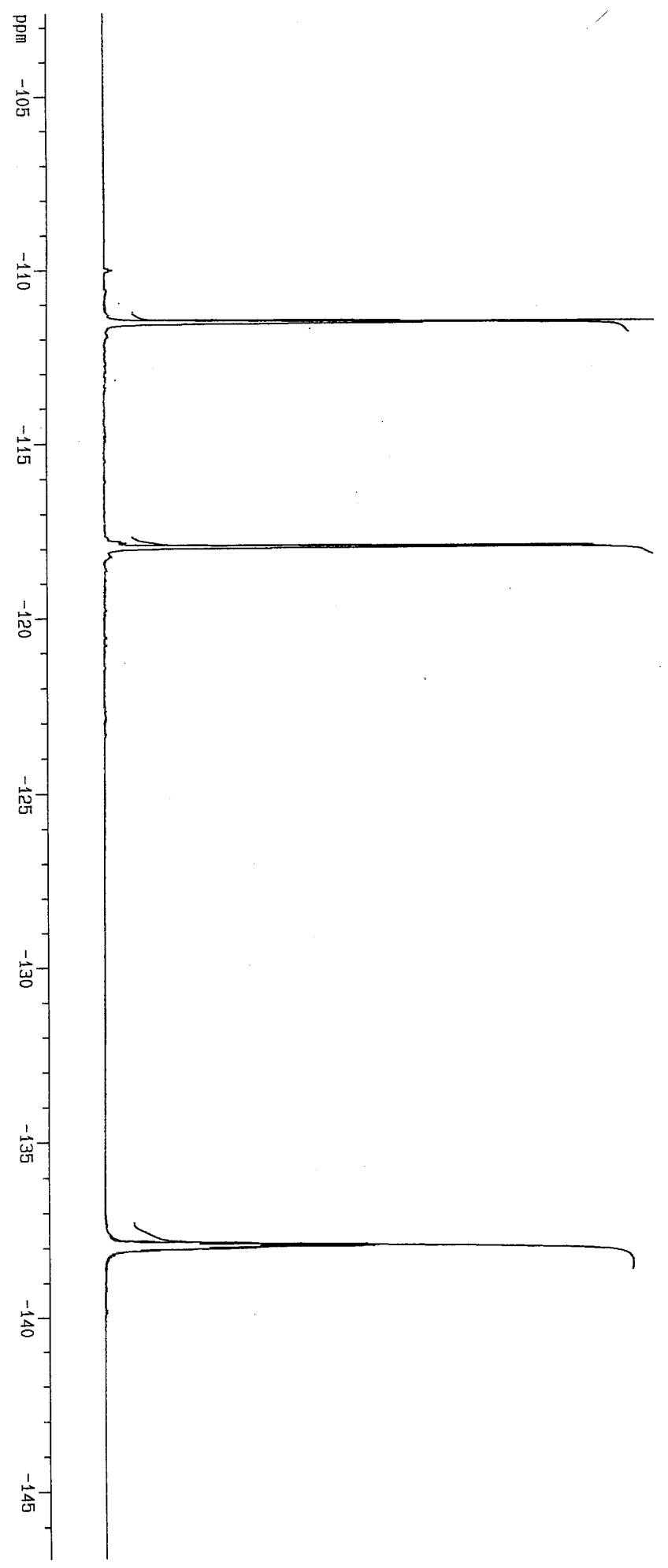

8

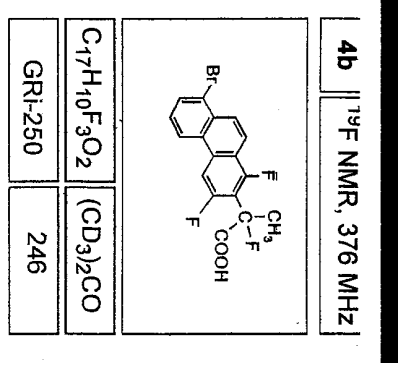



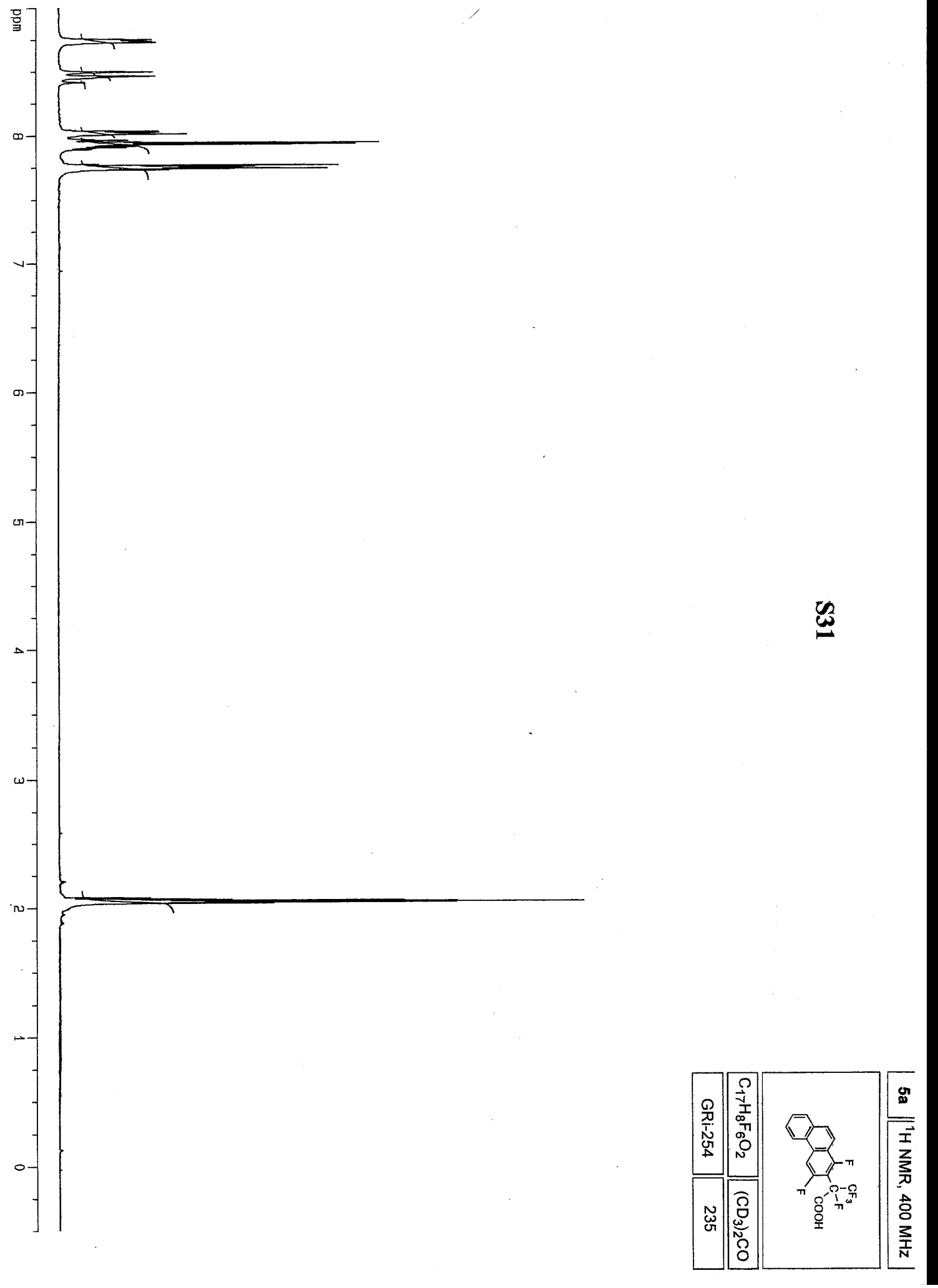

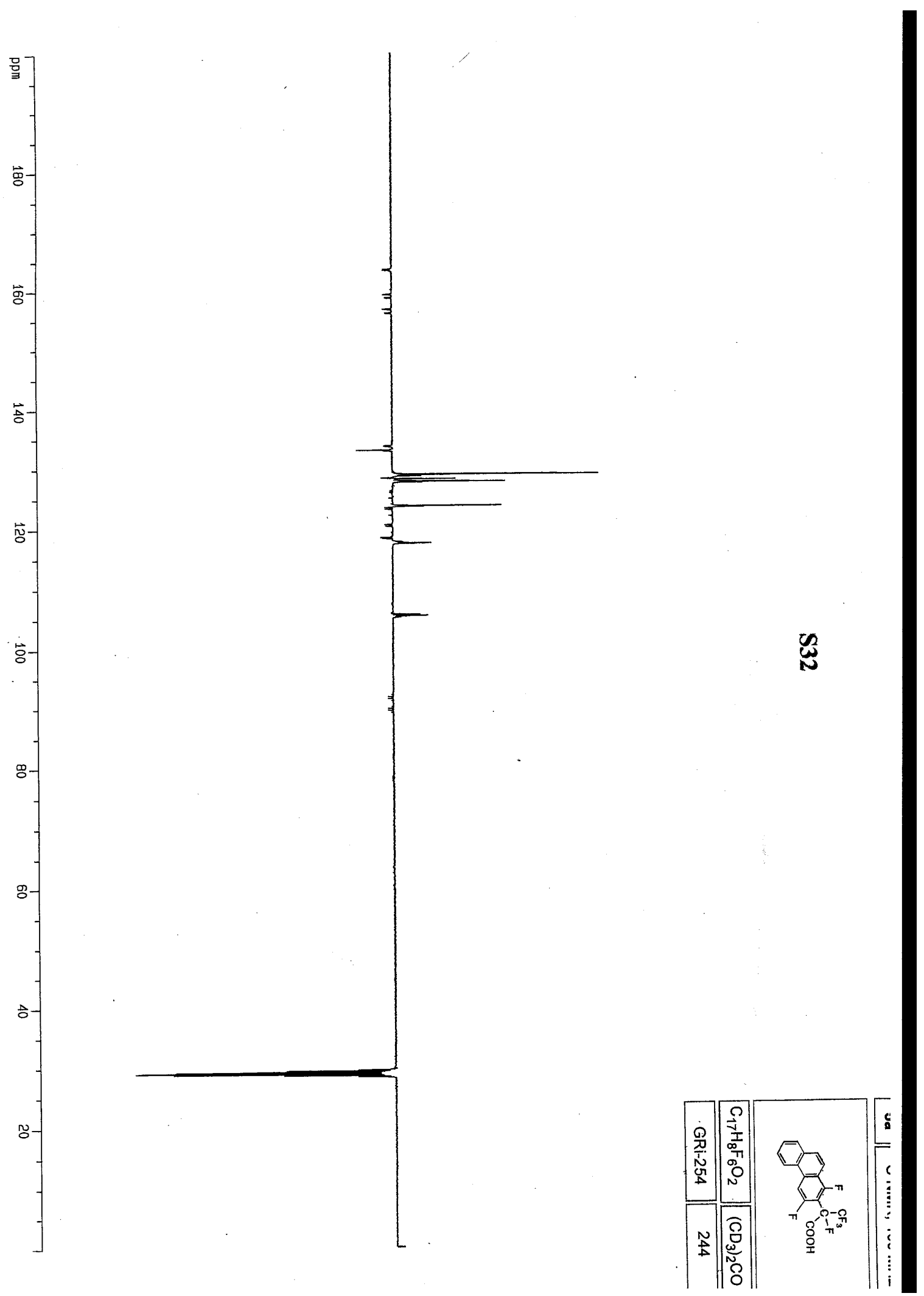


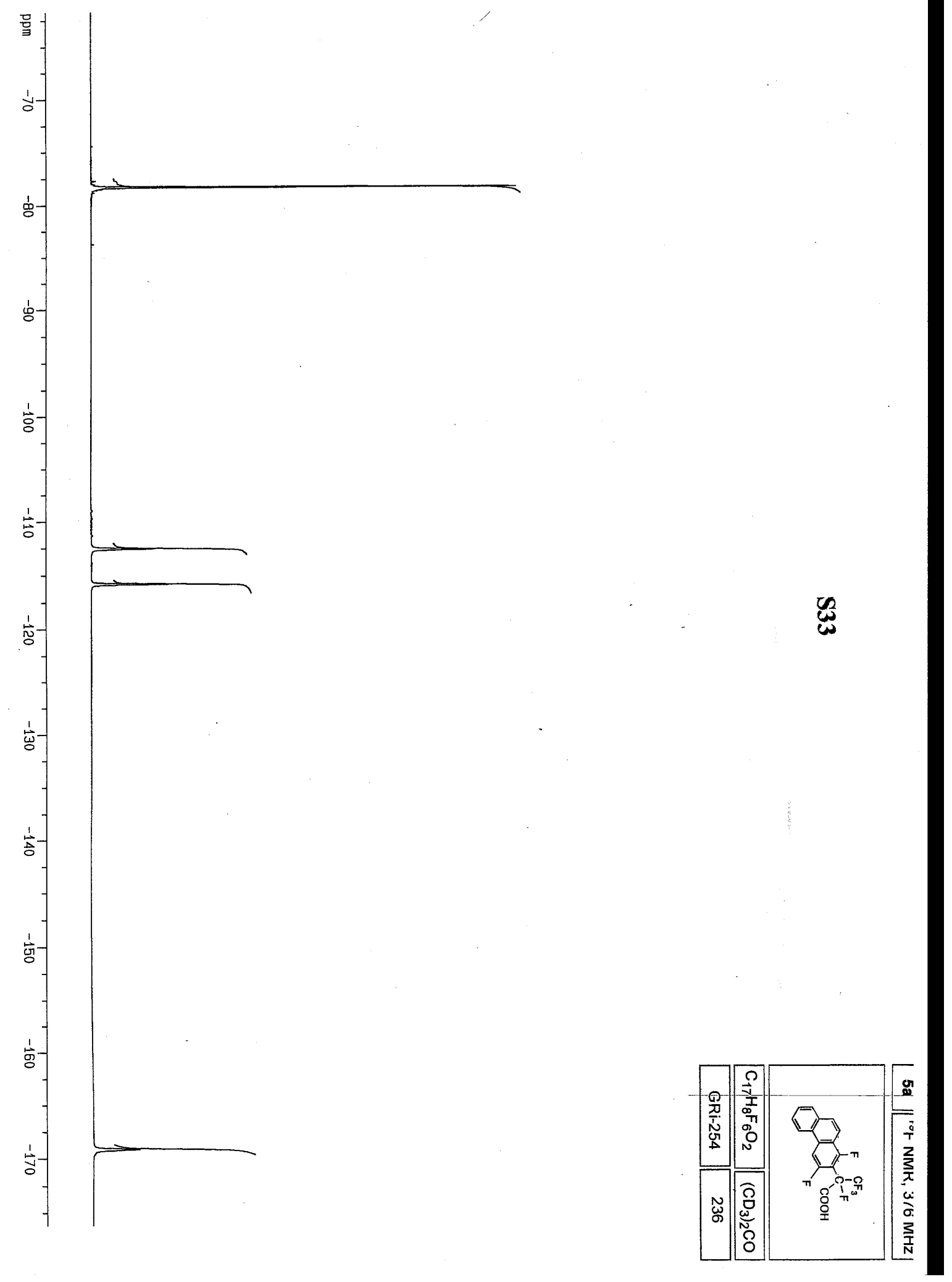




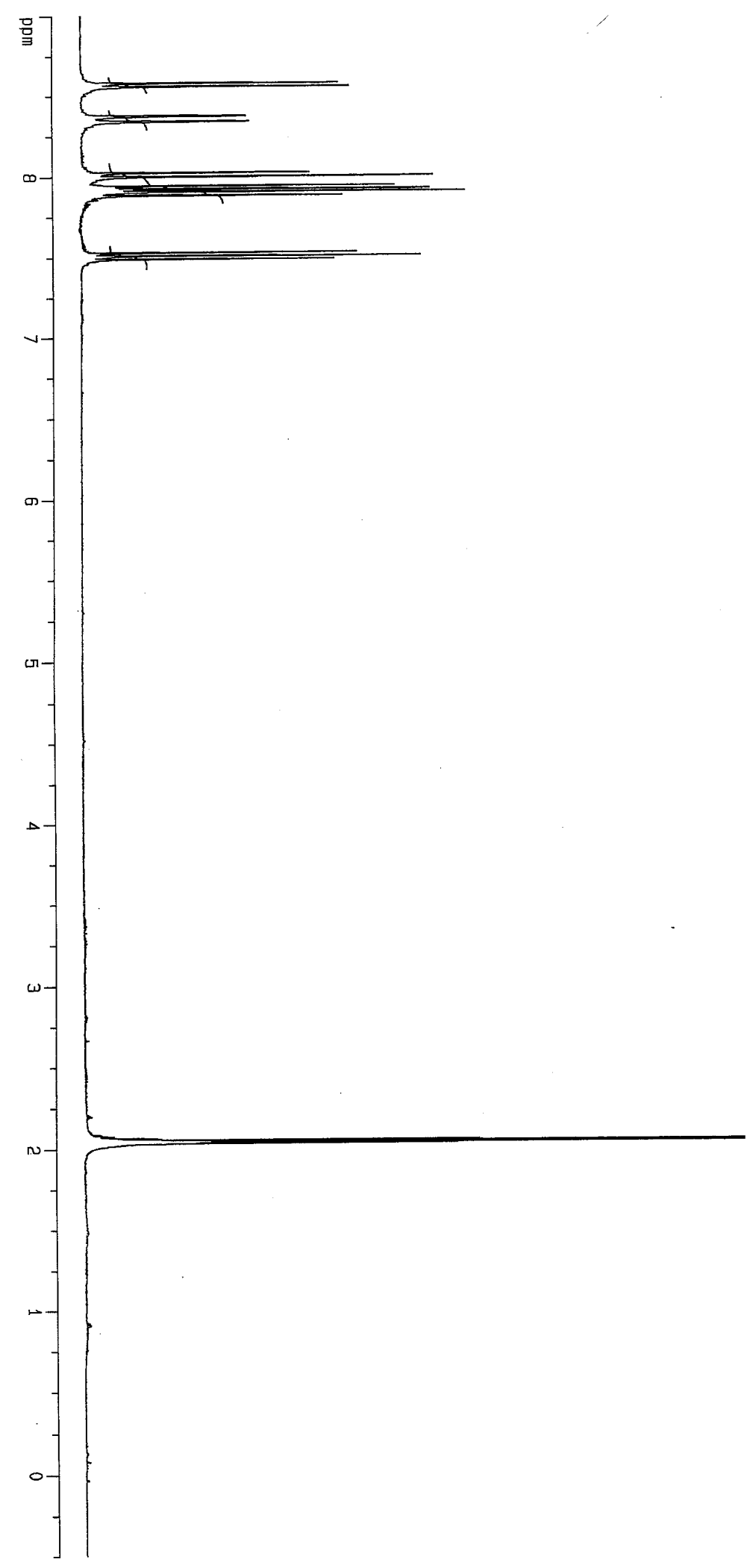

$\stackrel{5}{+}$

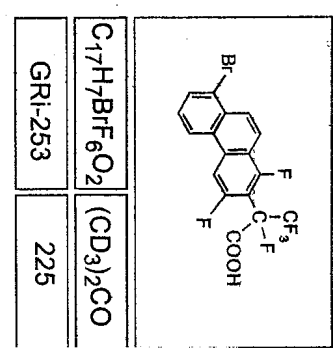




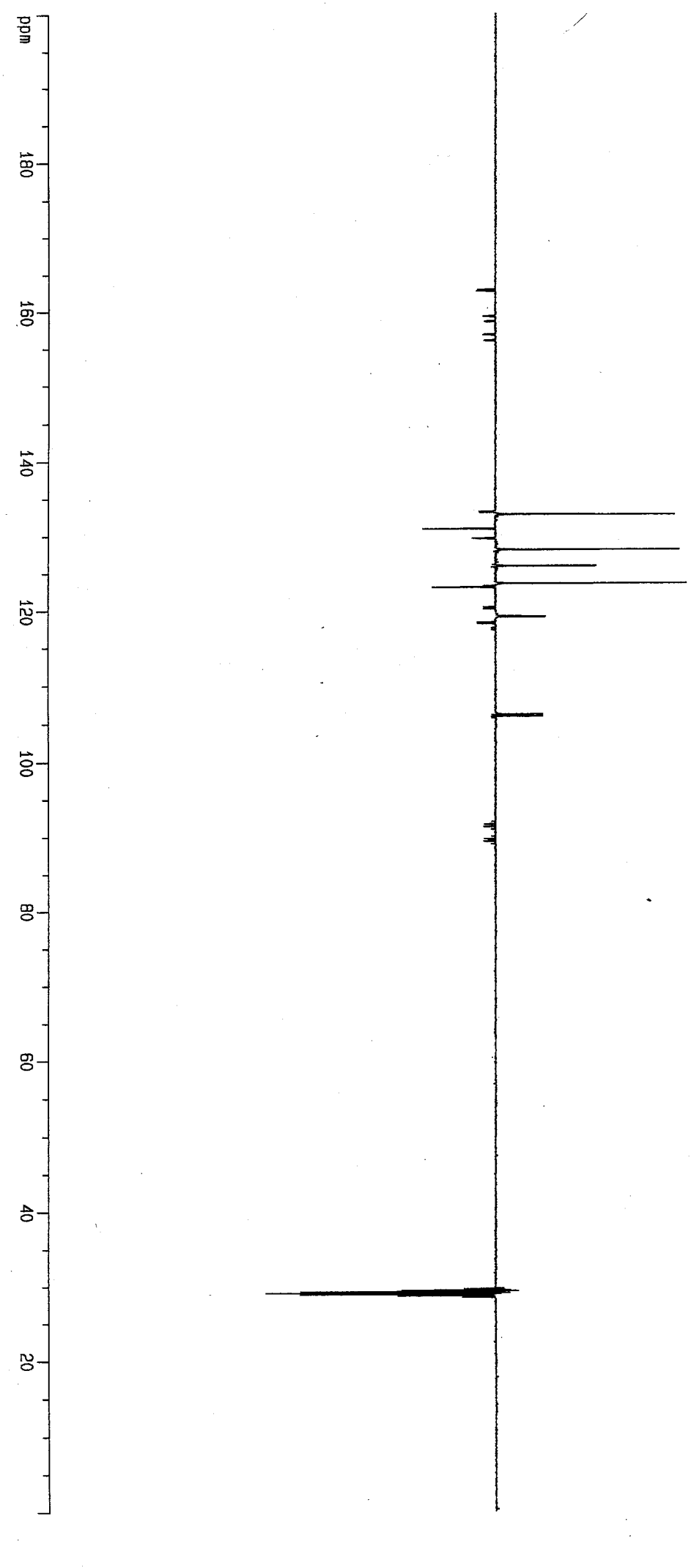

崫

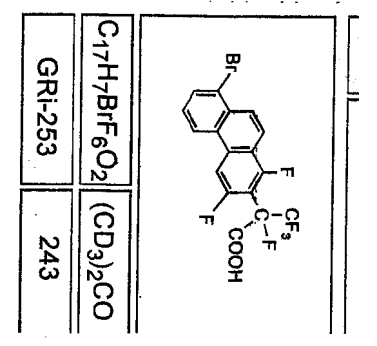




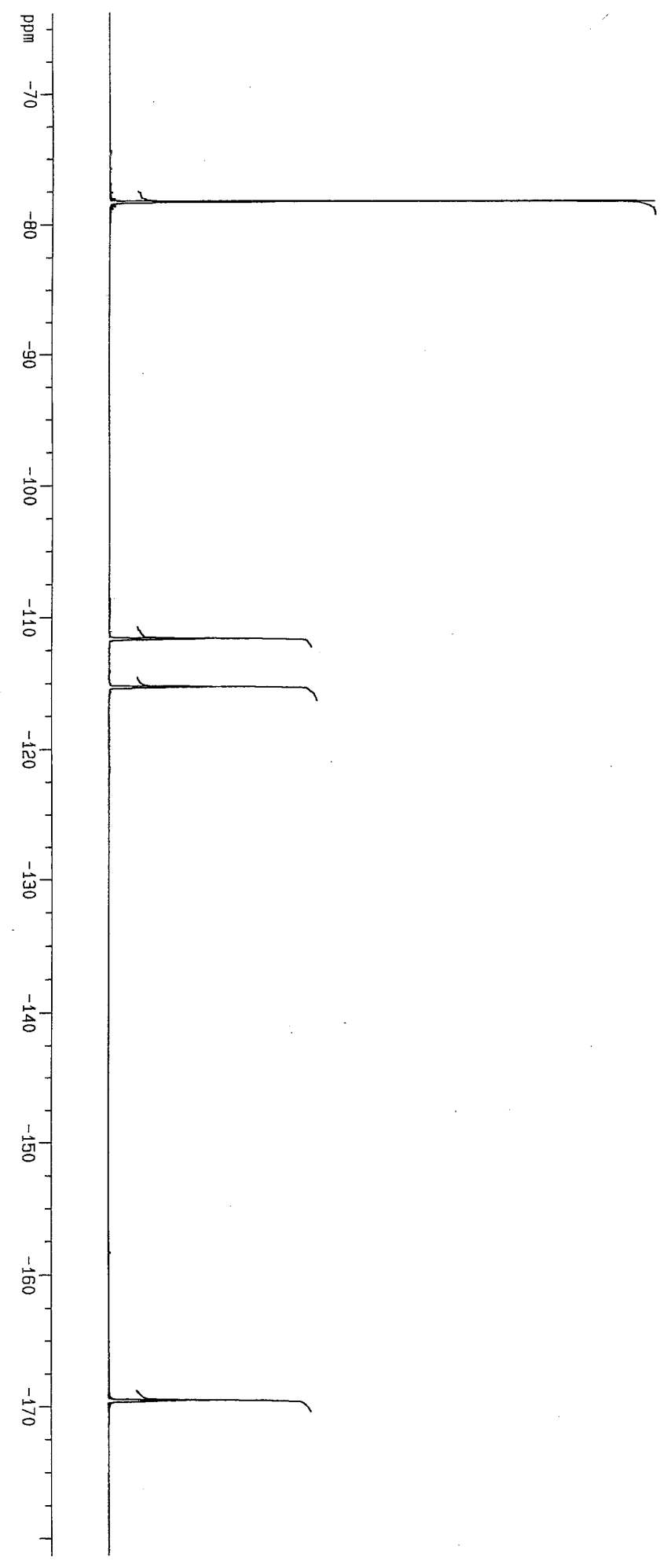

离

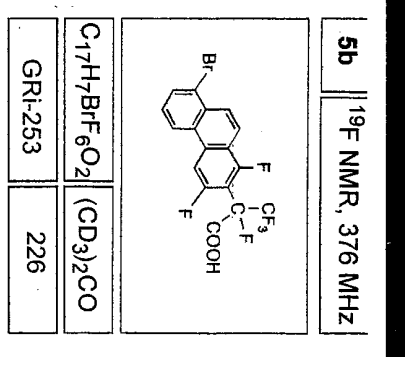




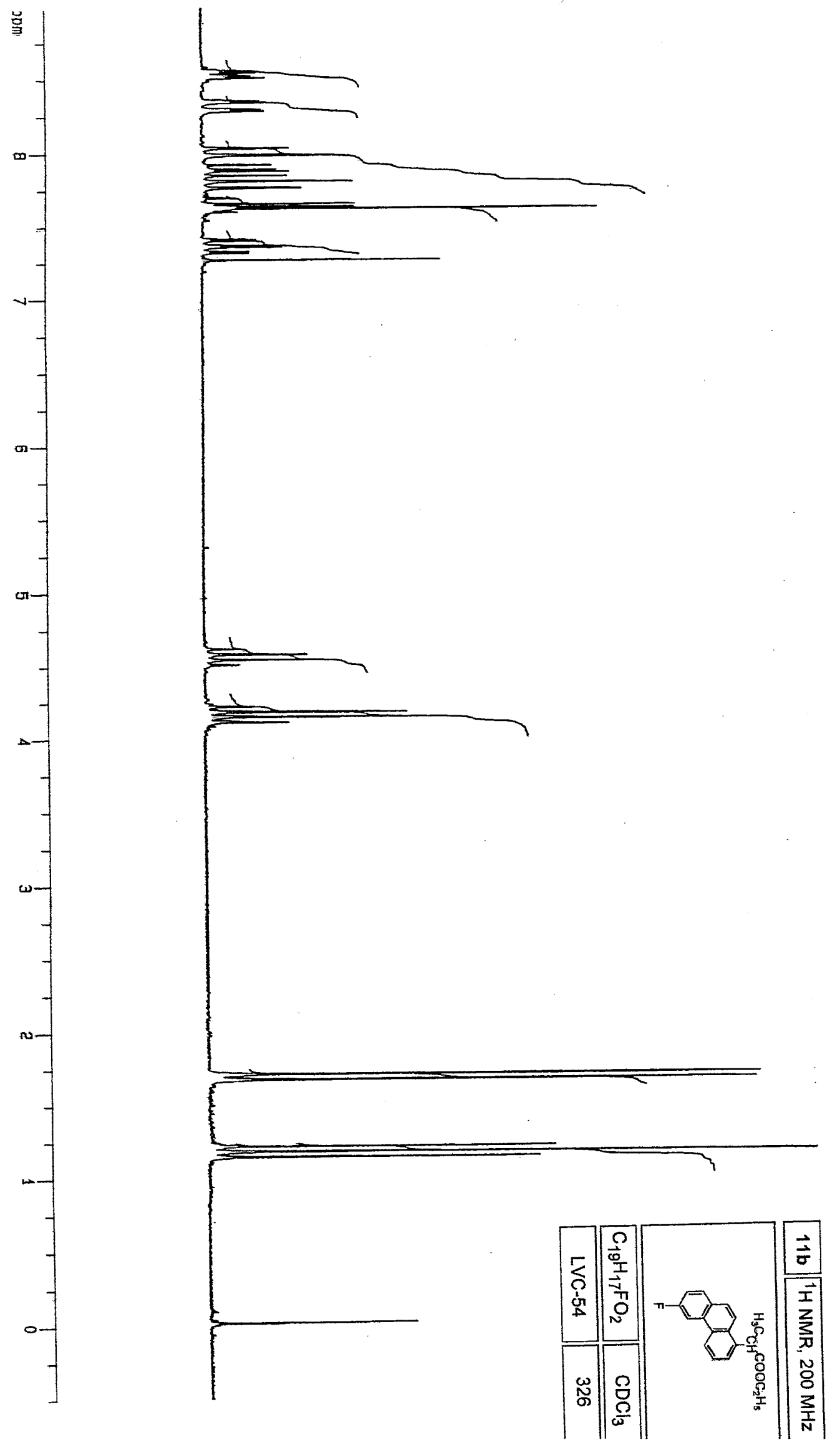




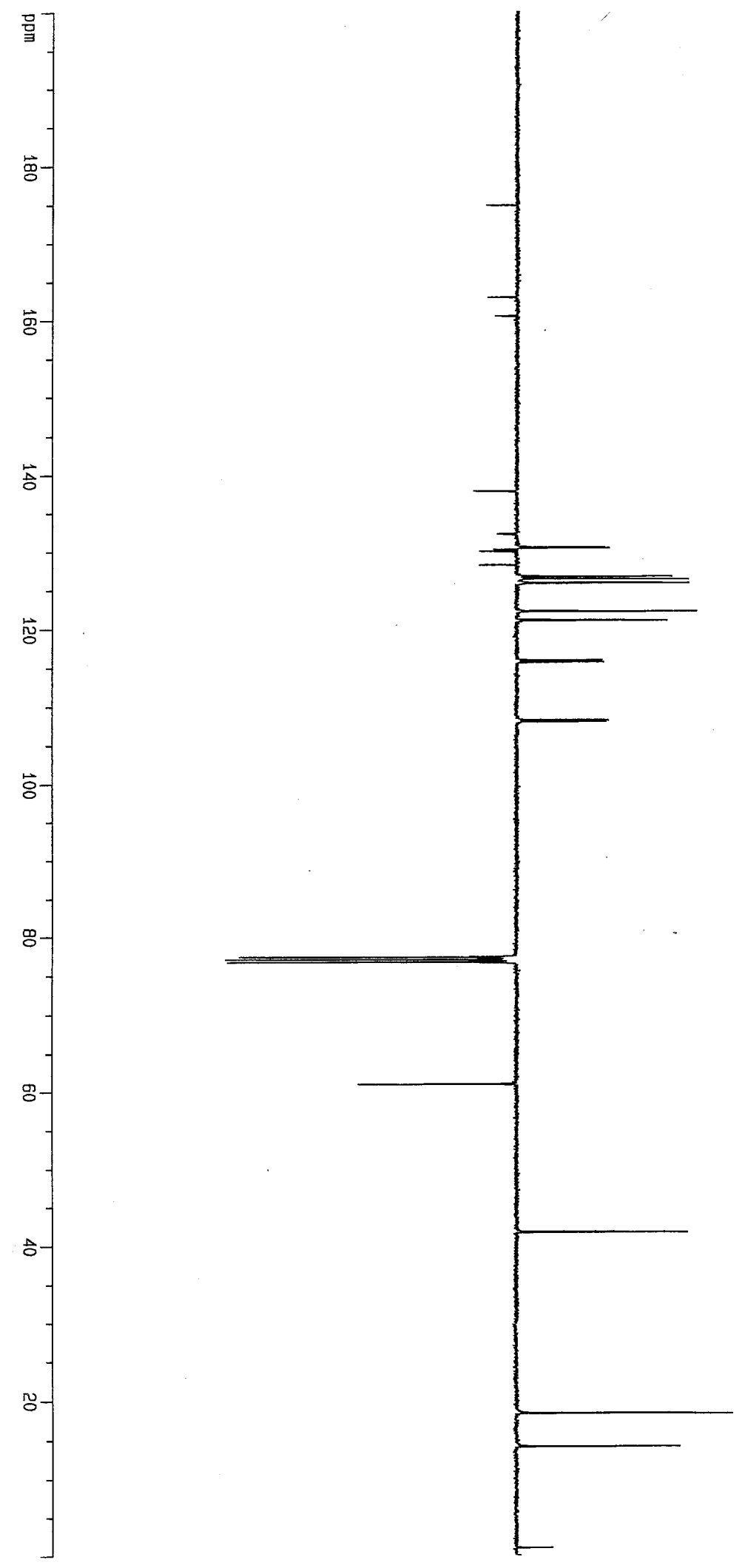

\%

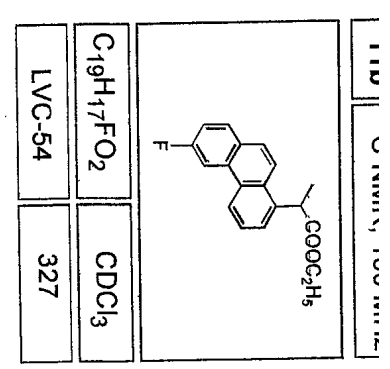




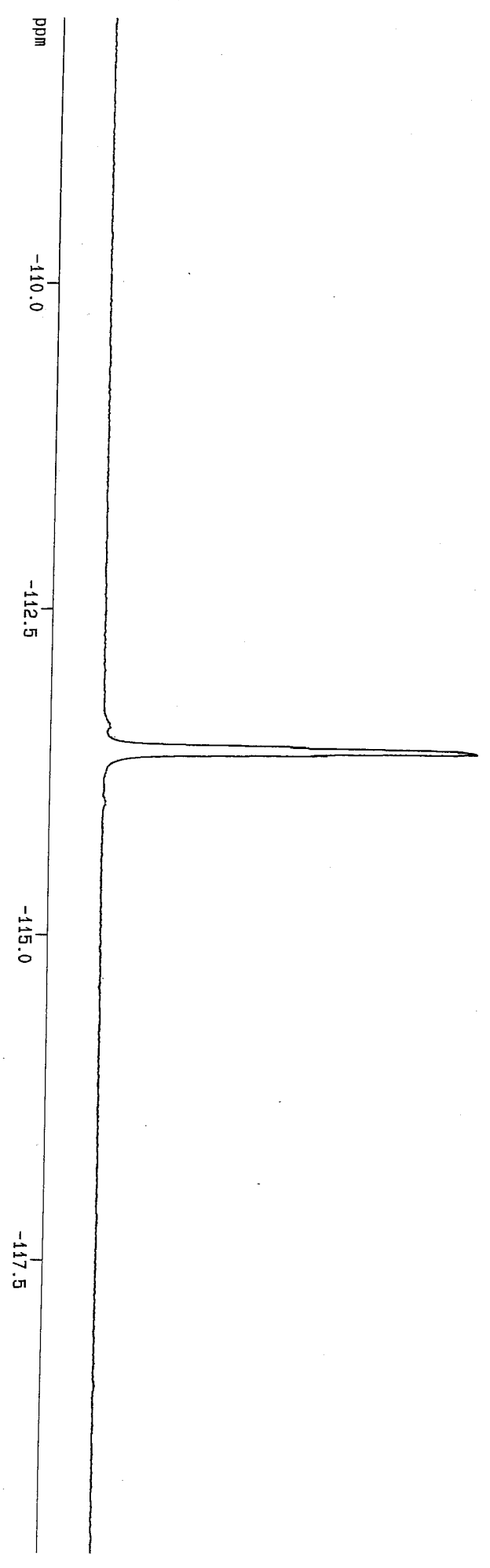

造

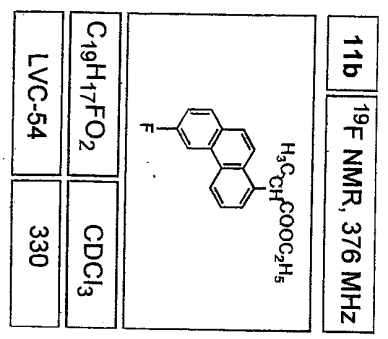




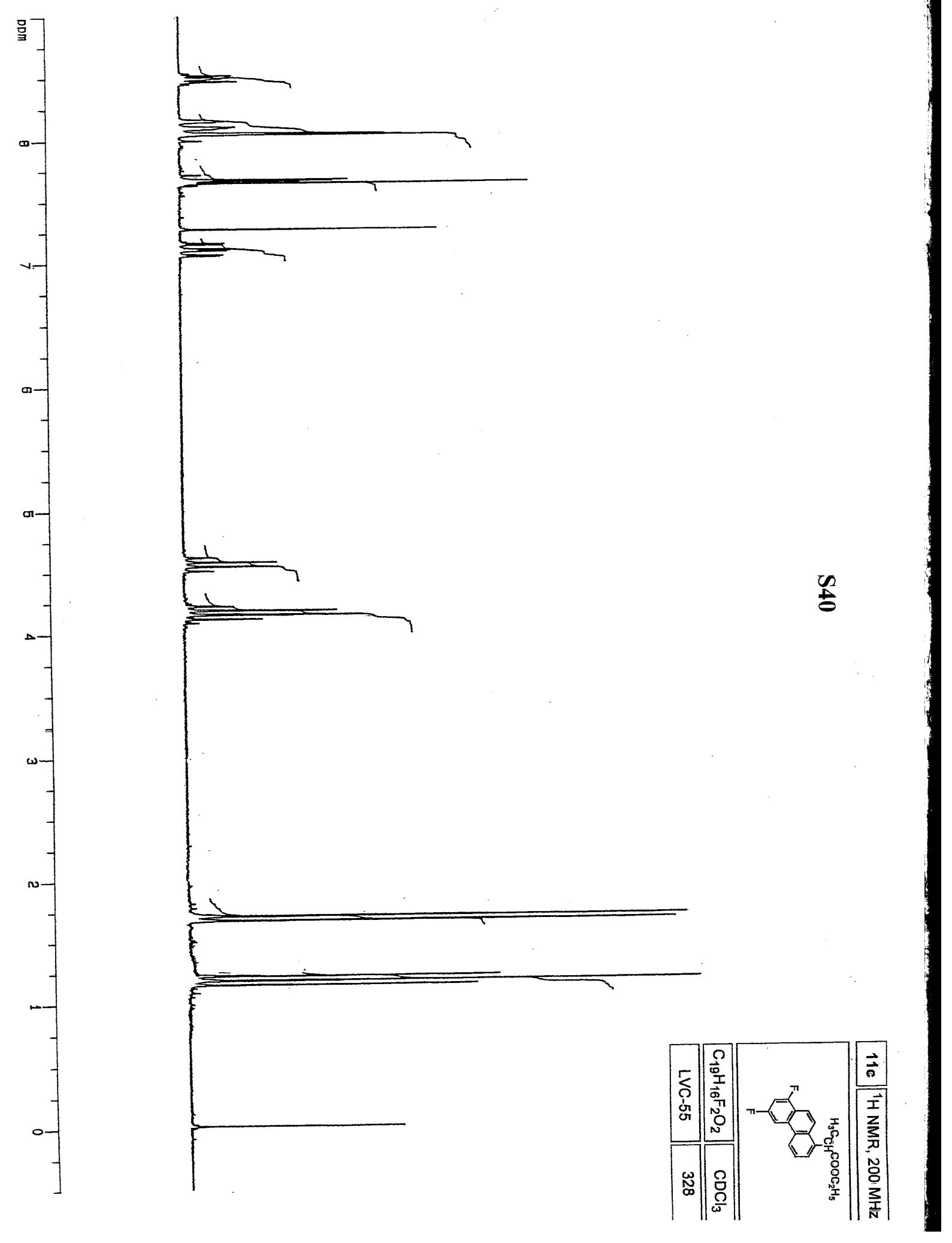



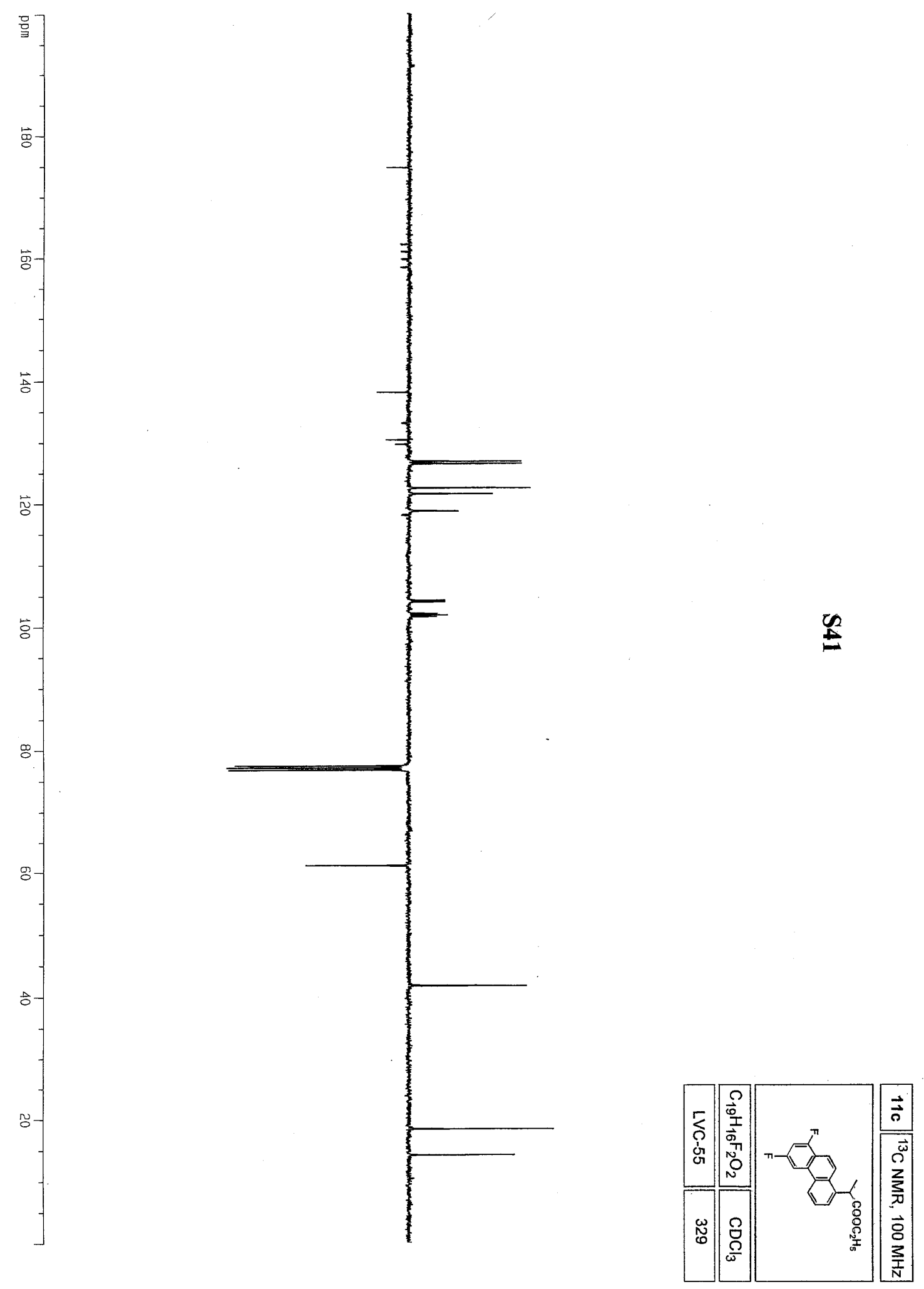

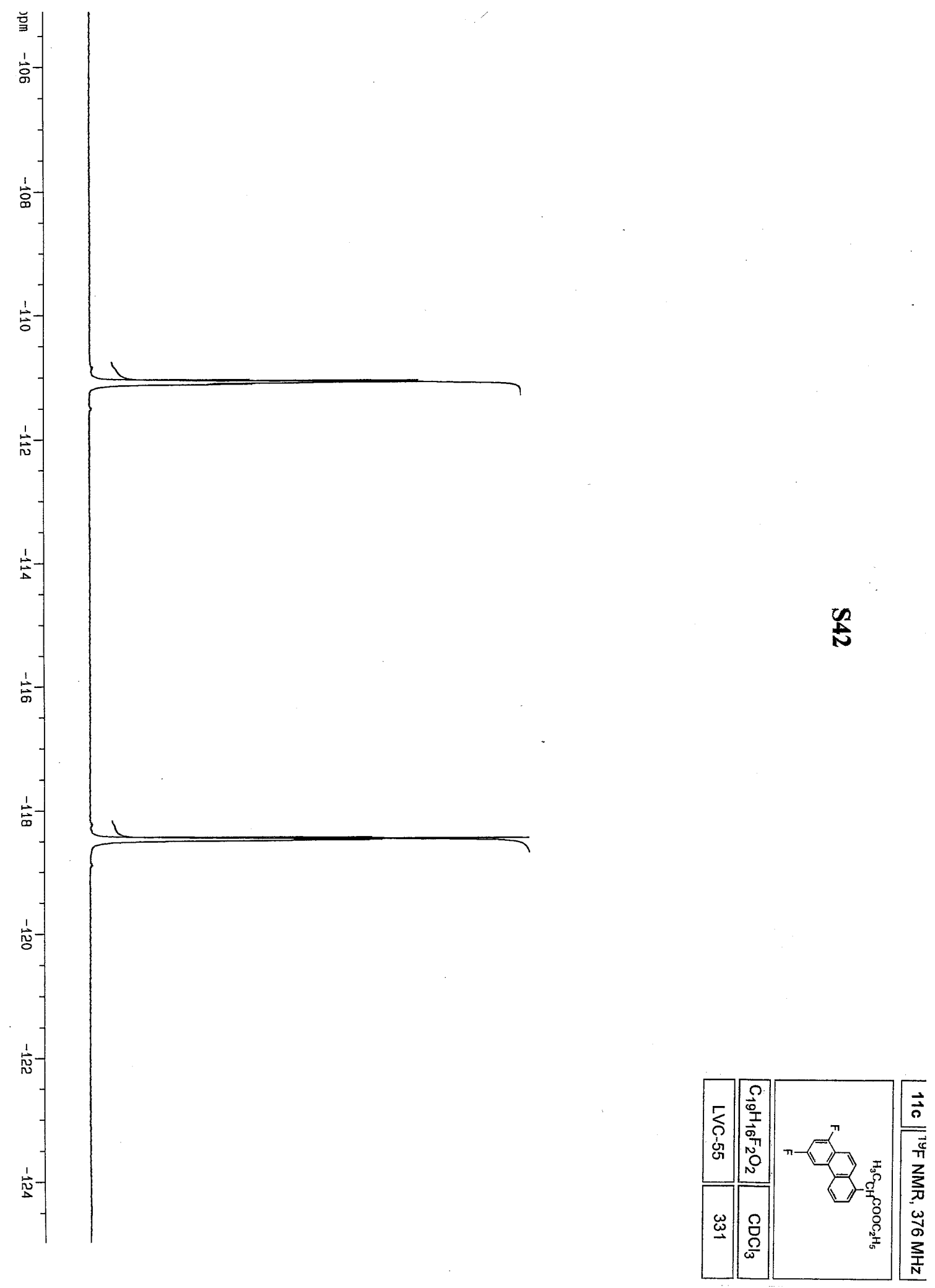

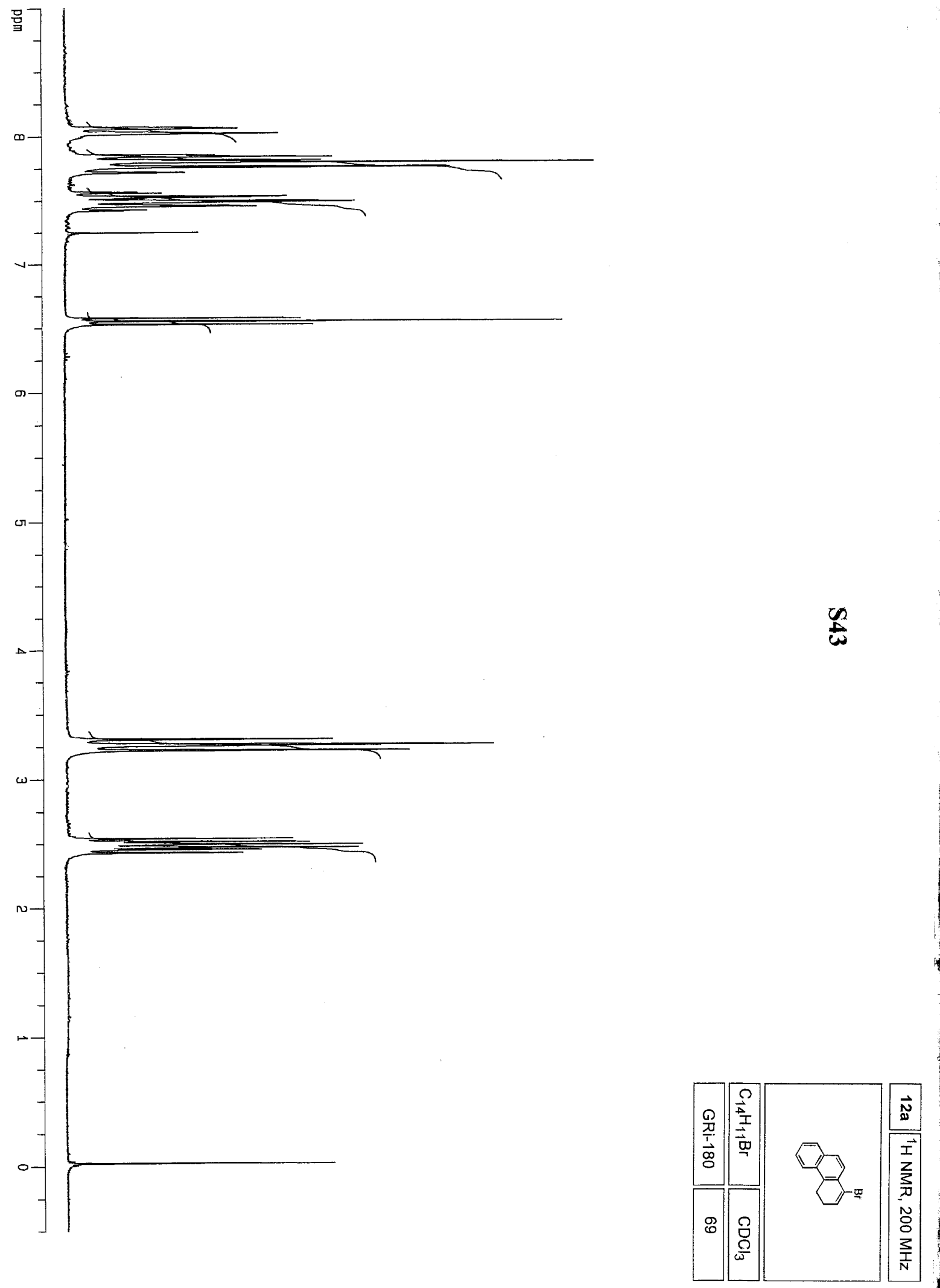


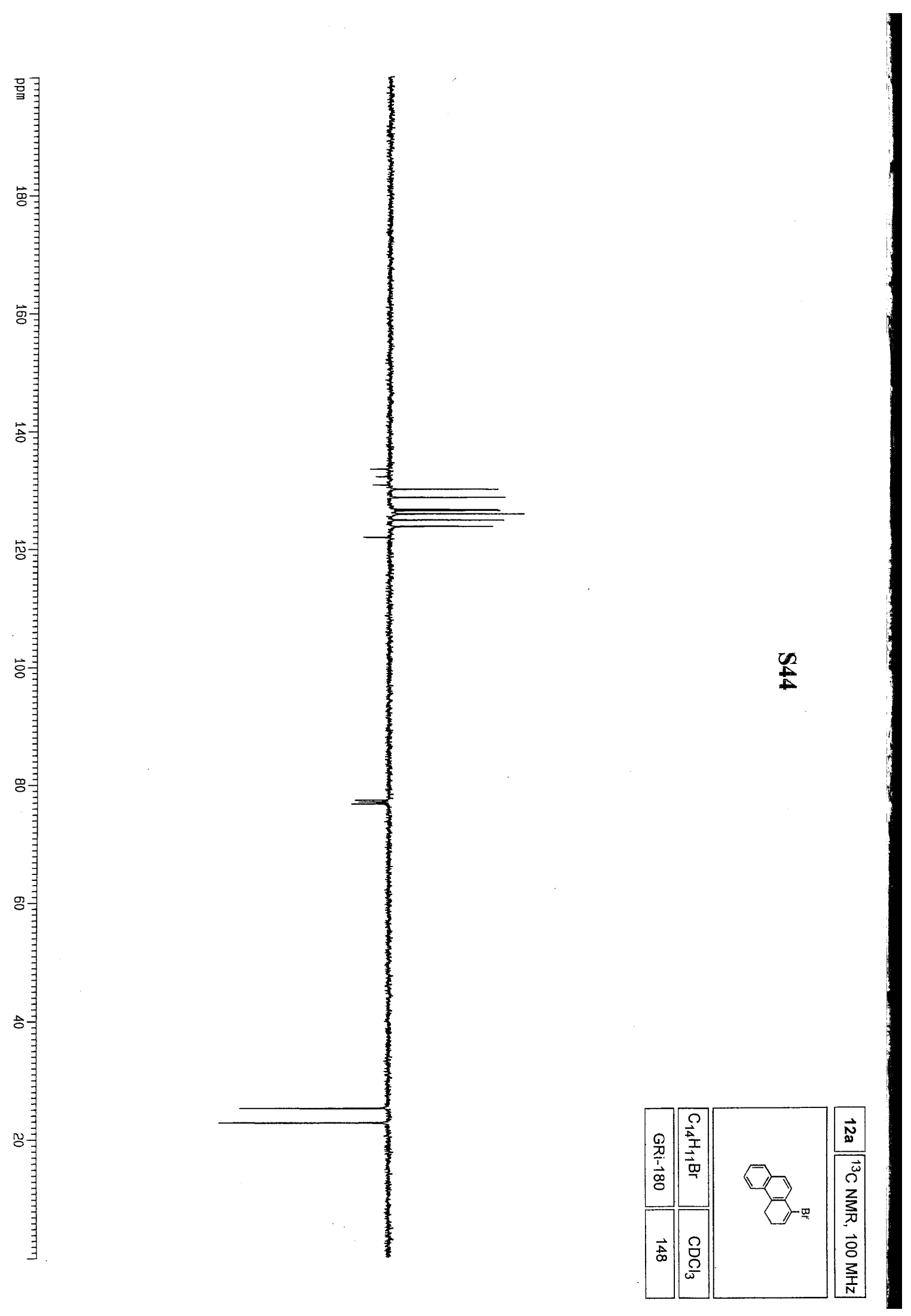



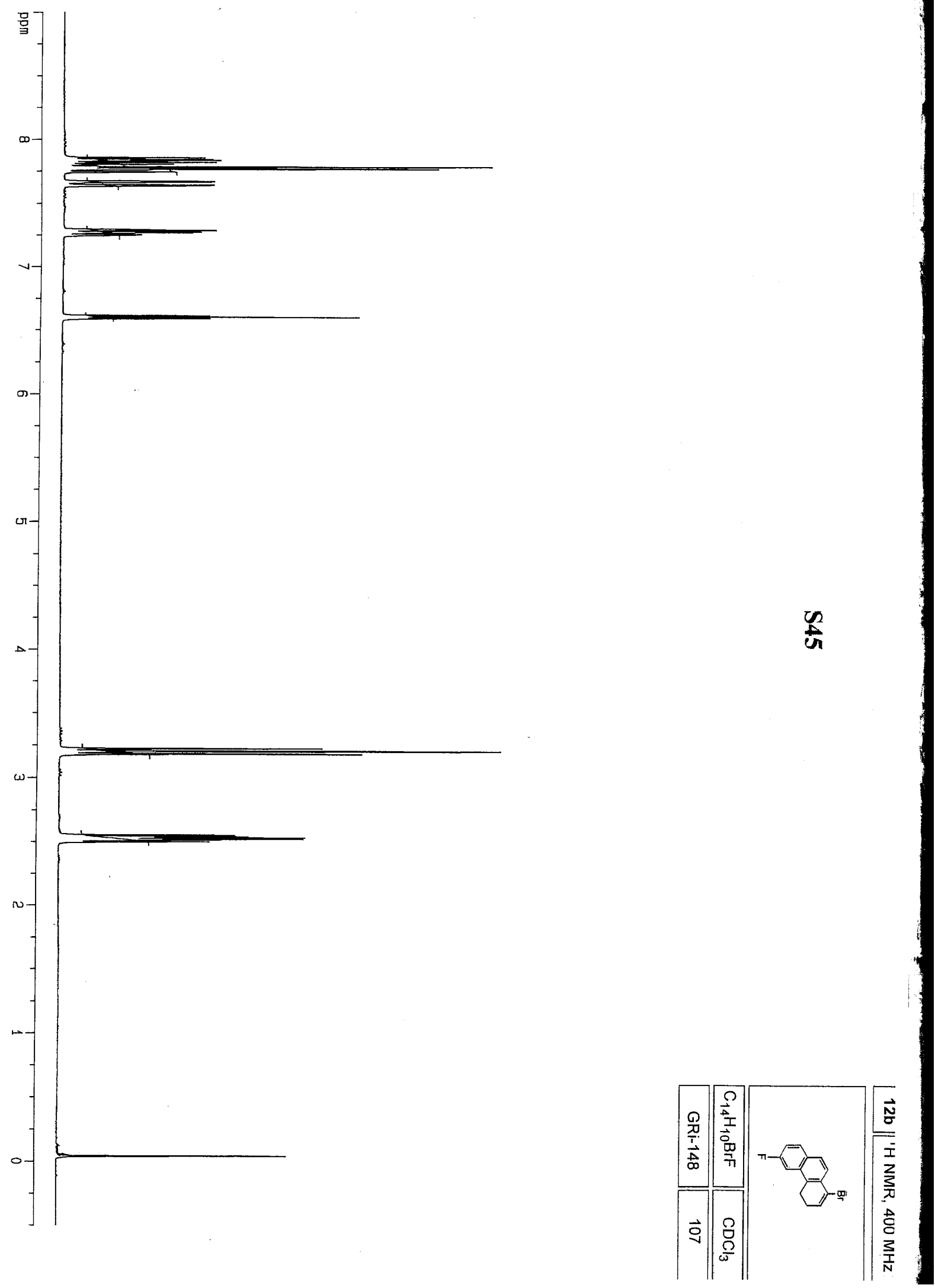

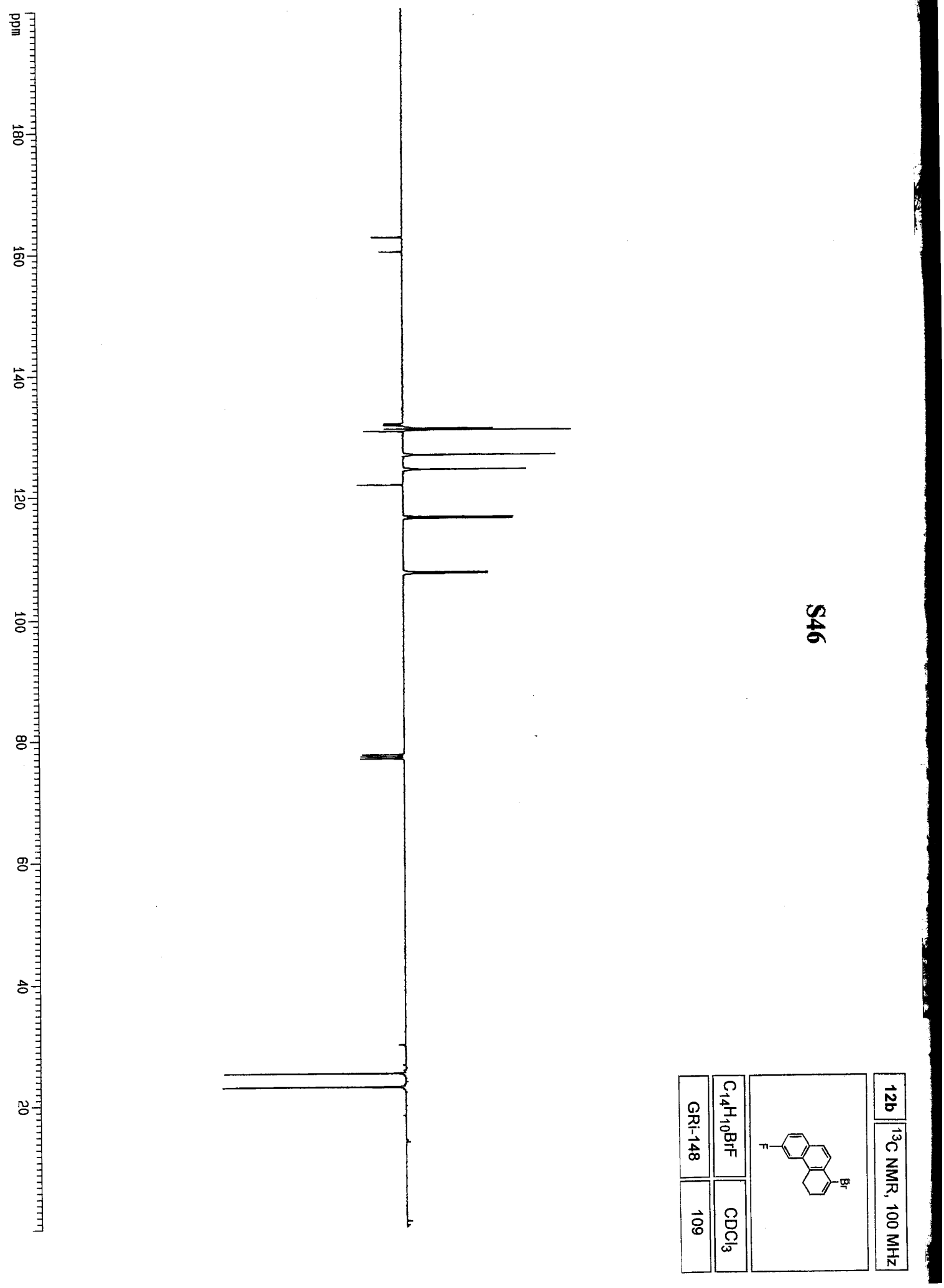


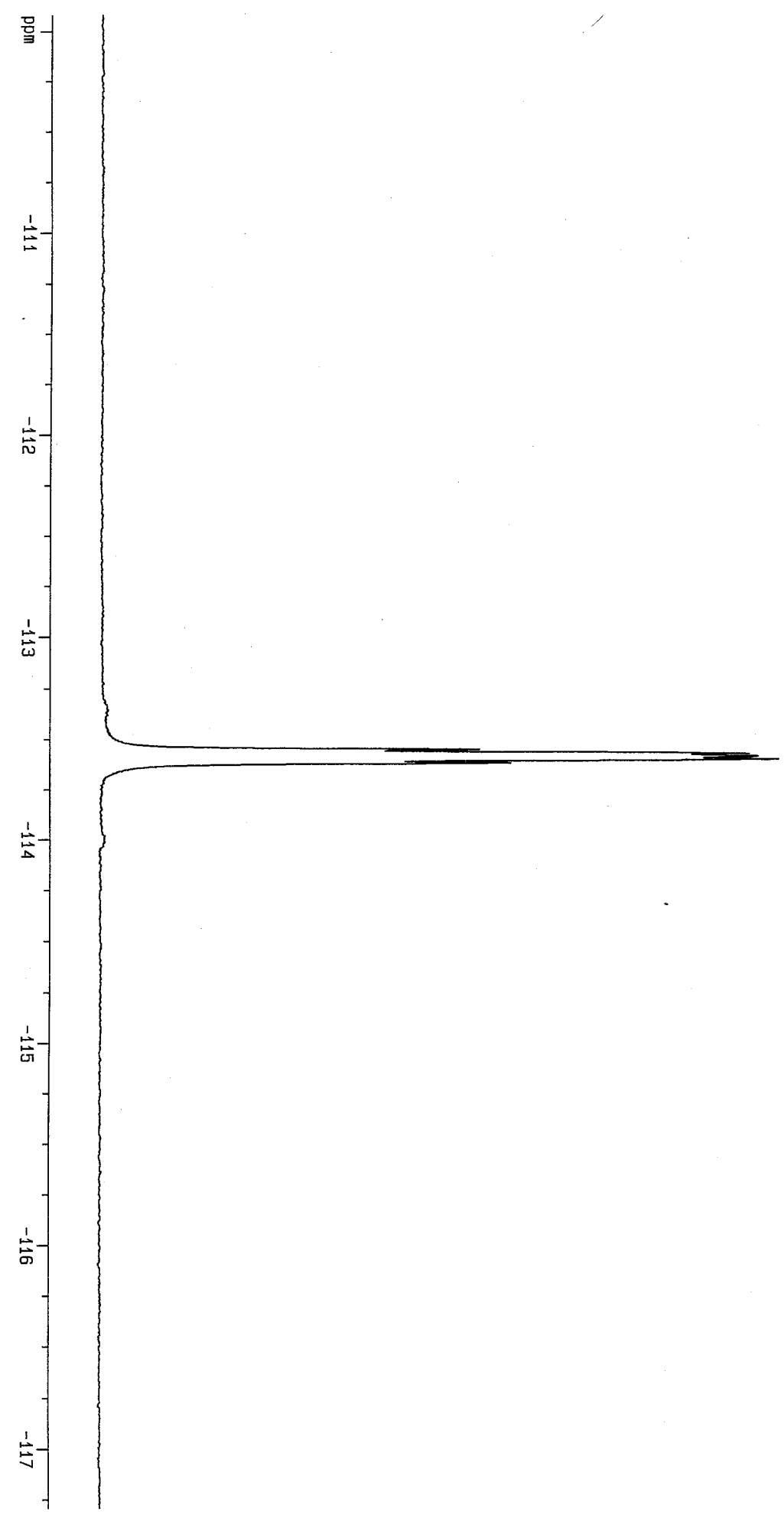




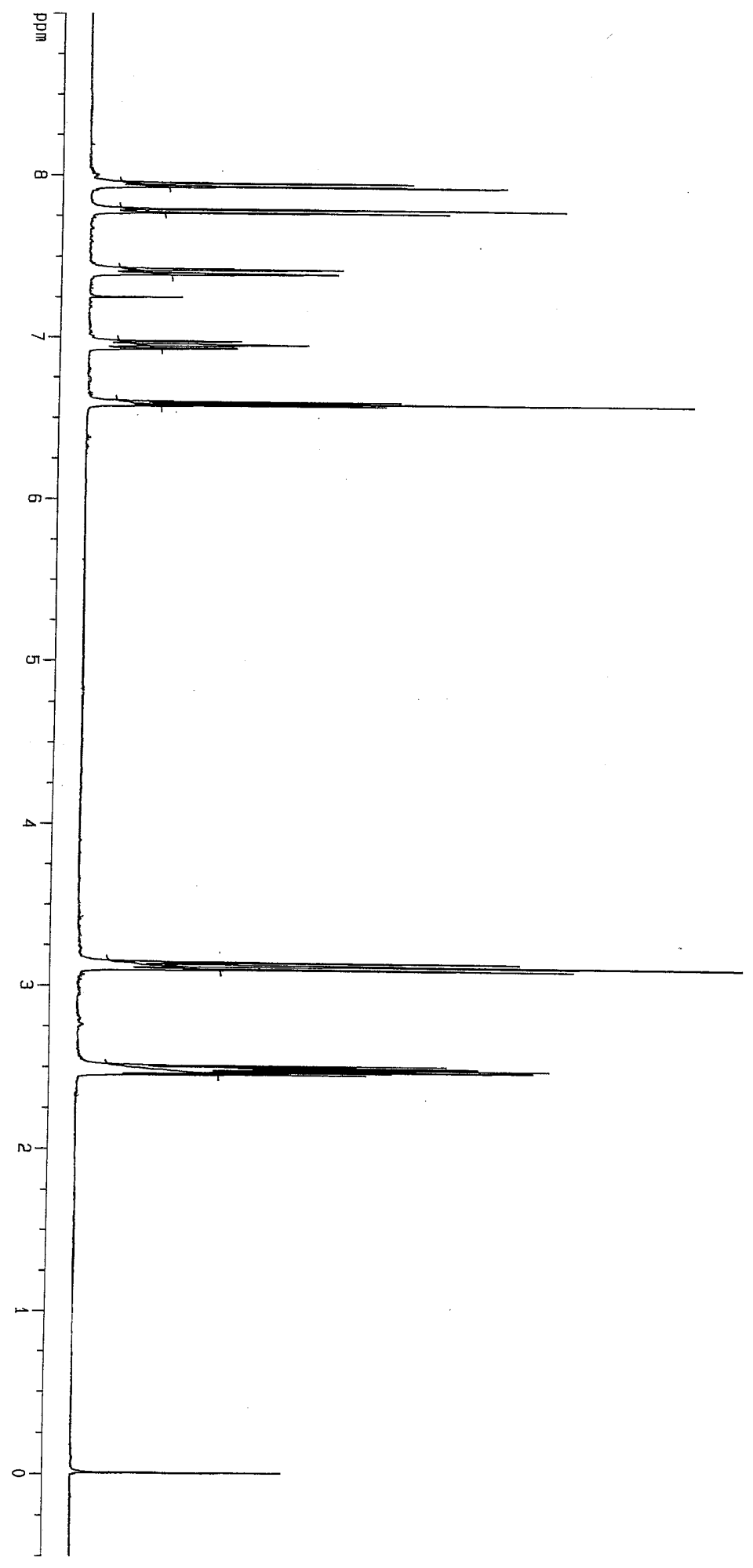

$\frac{5}{\infty}$

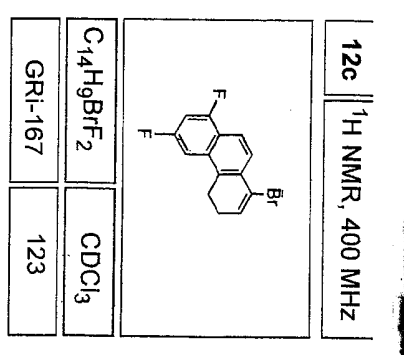



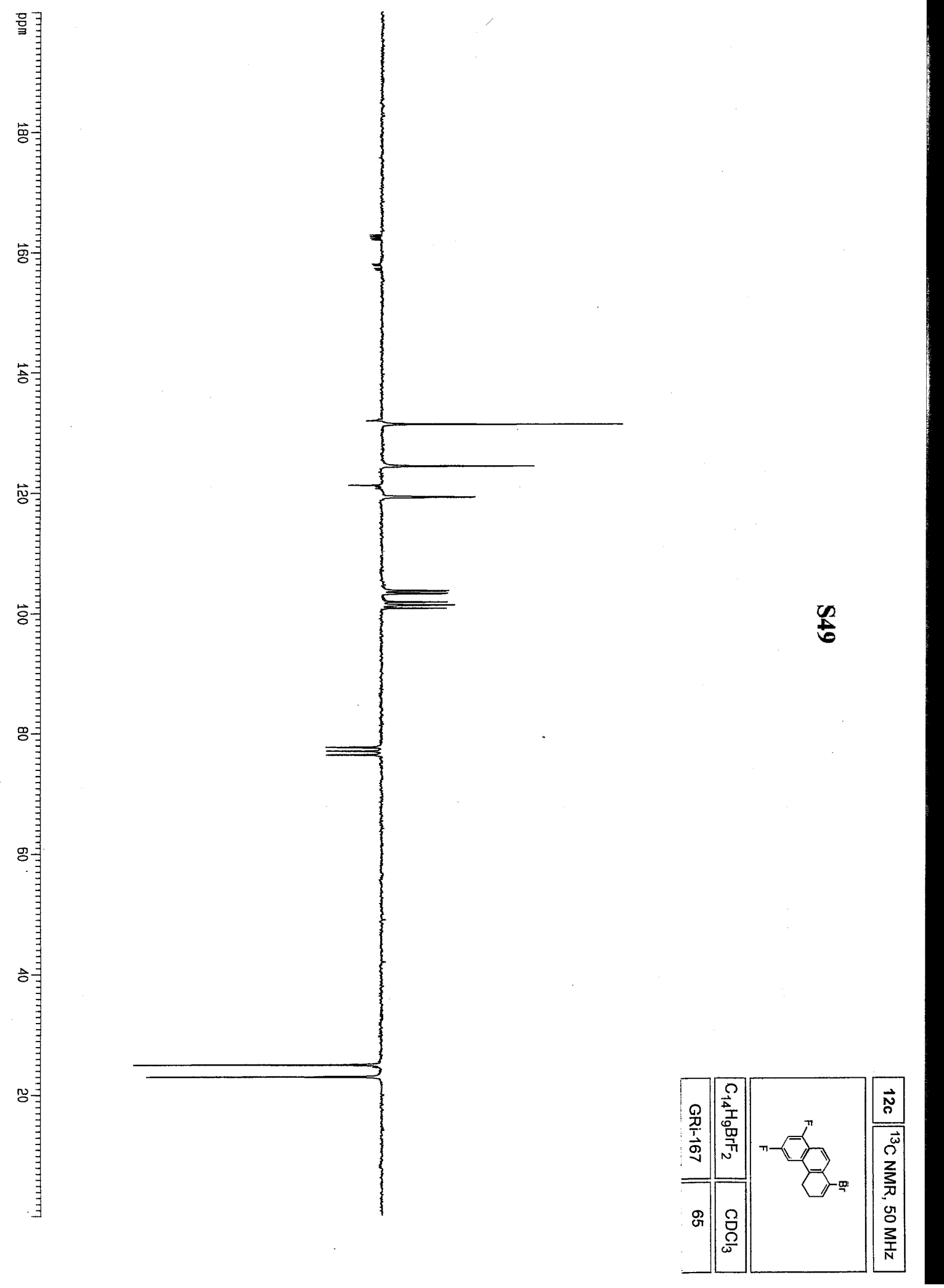


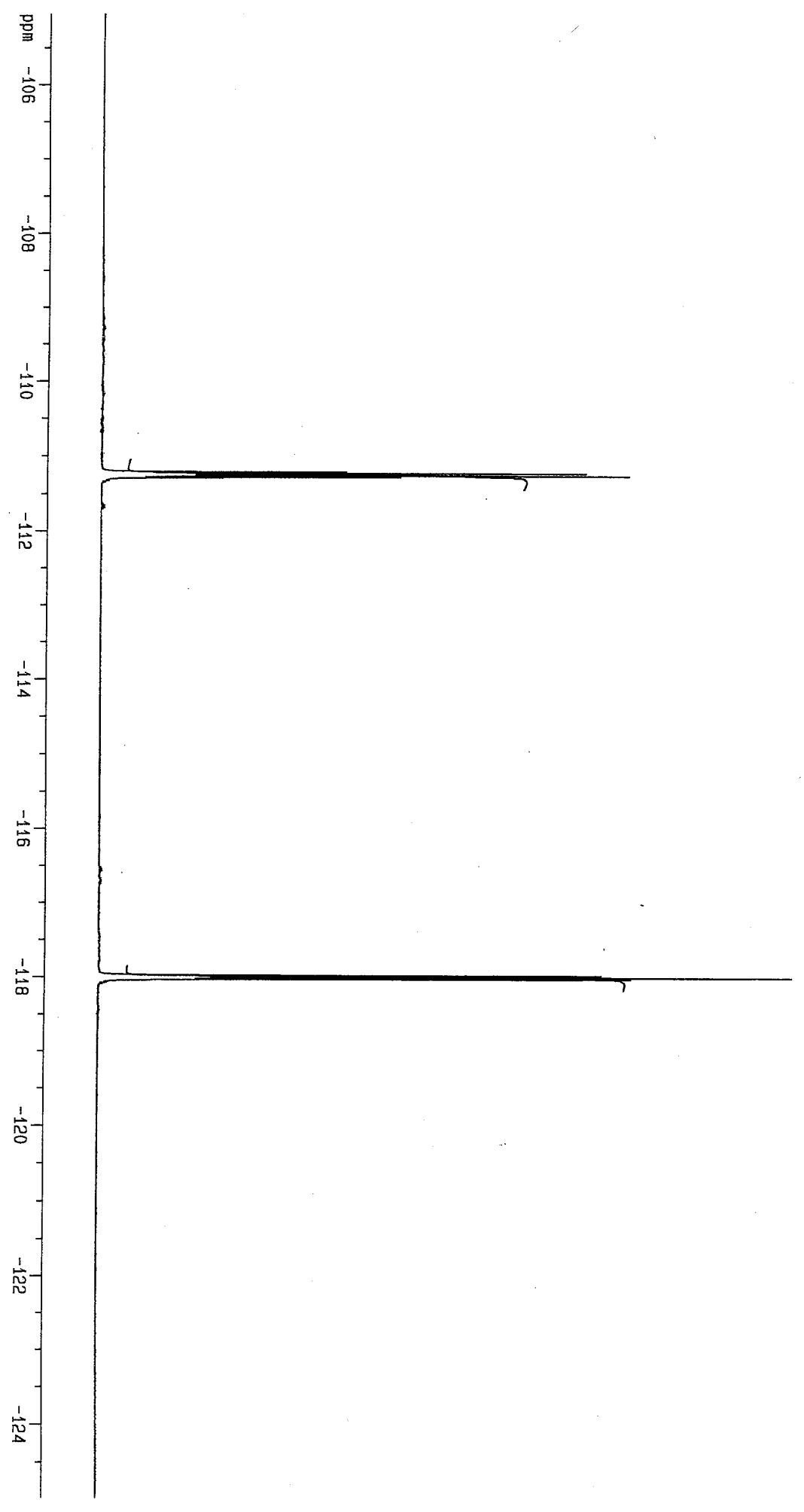

棺

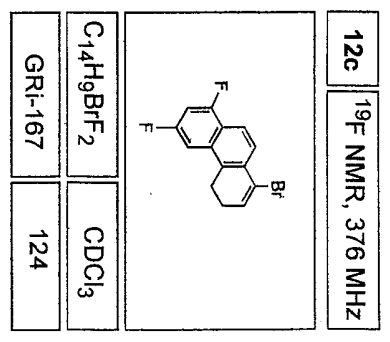



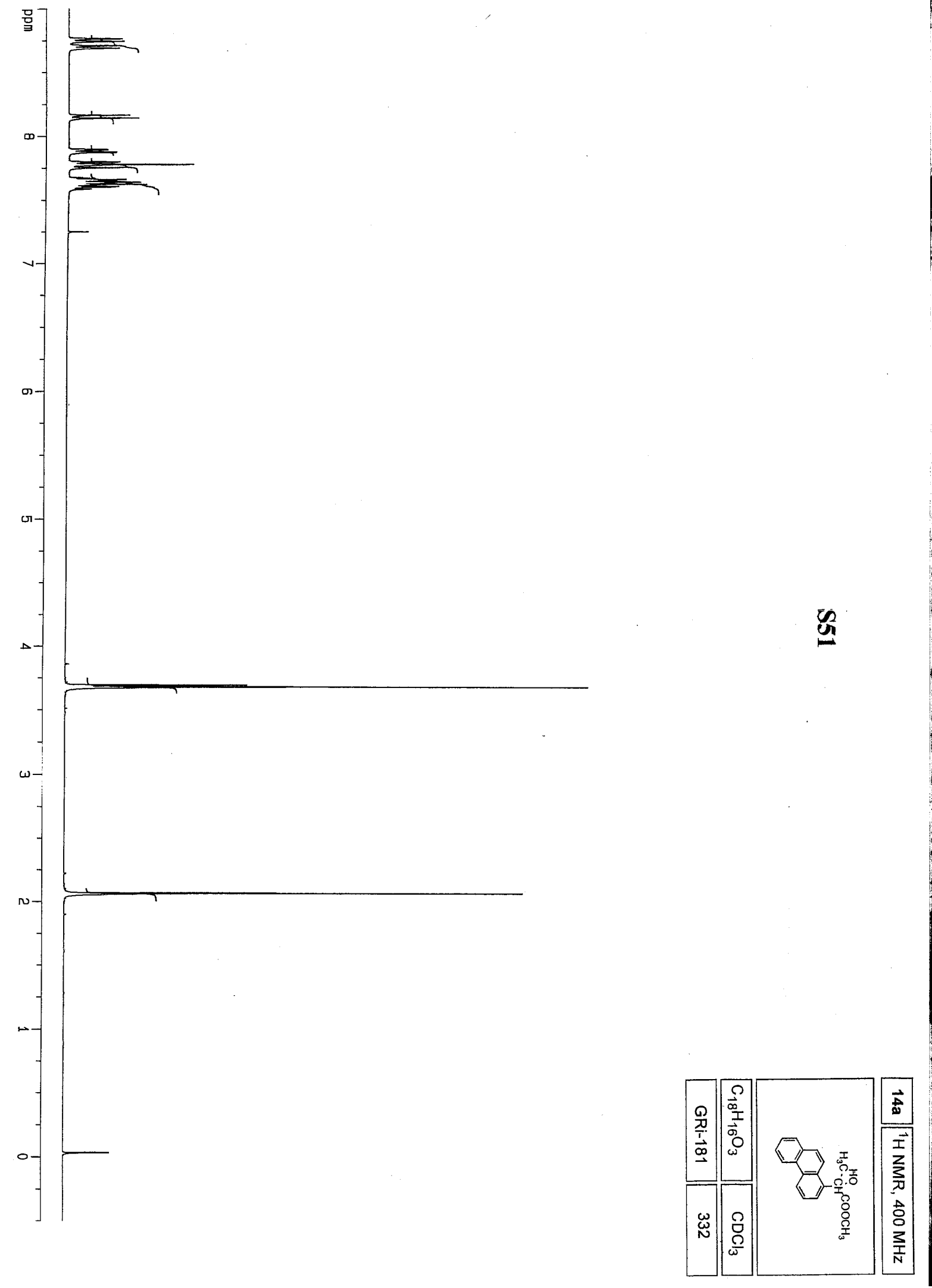

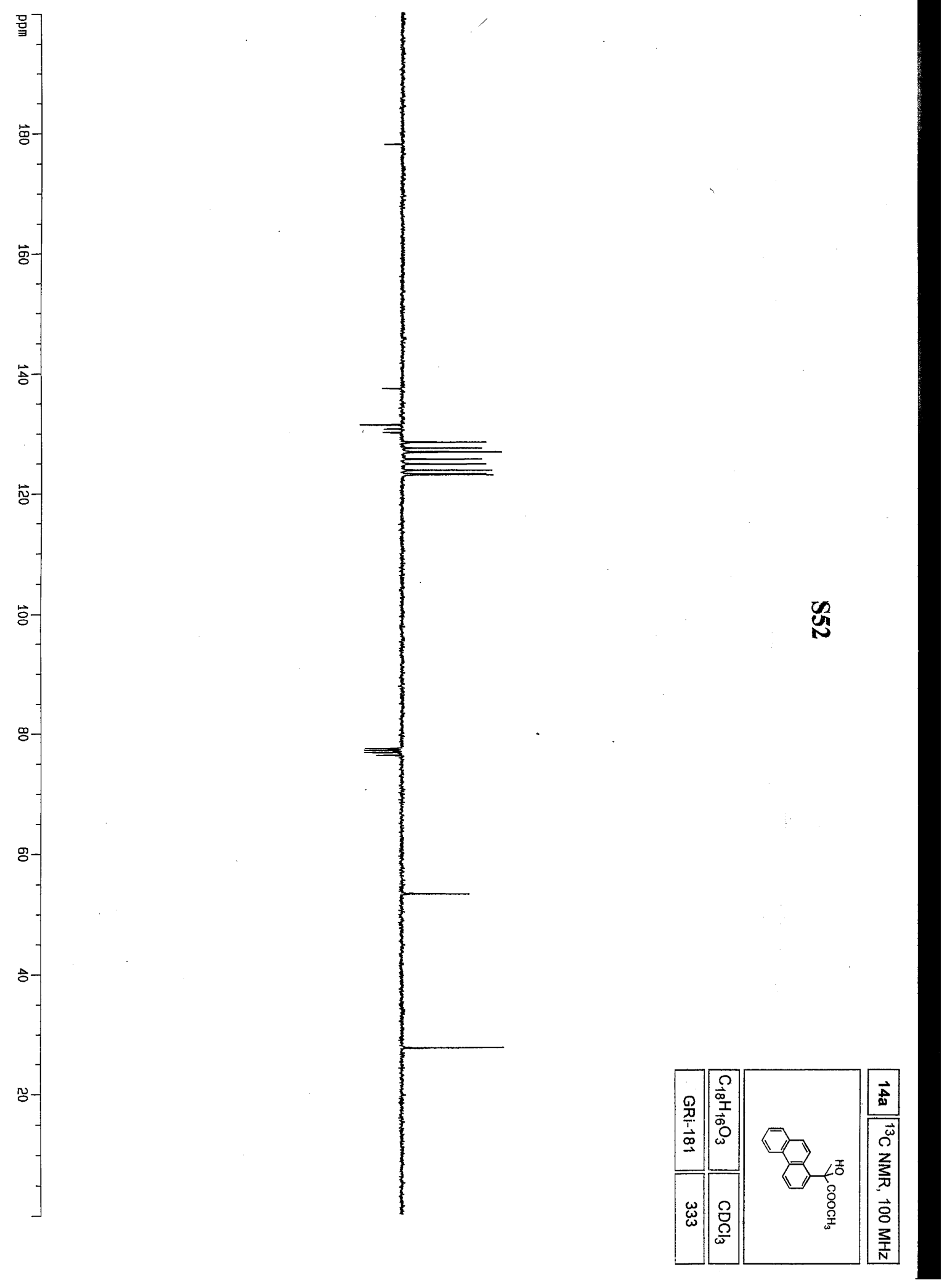

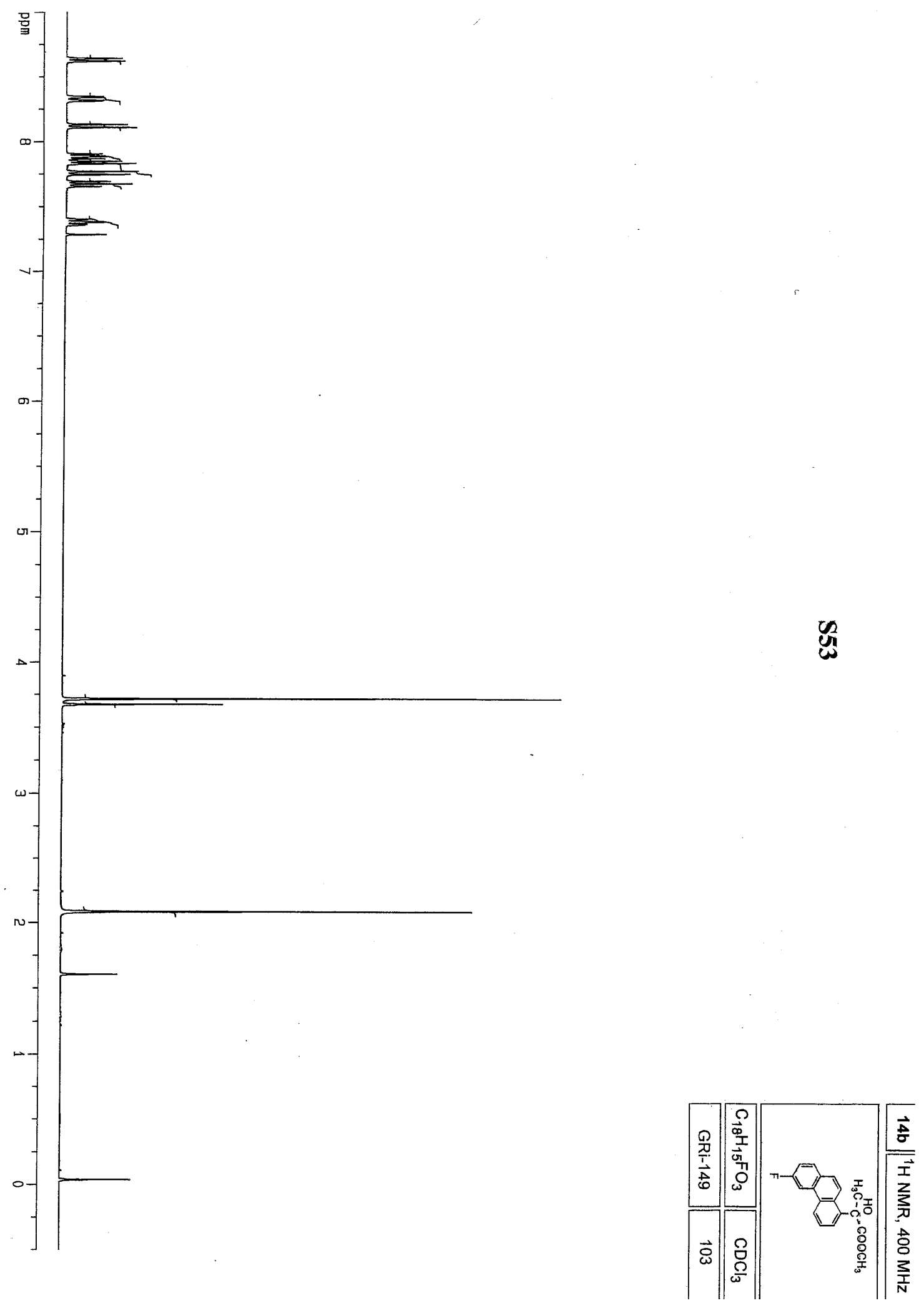


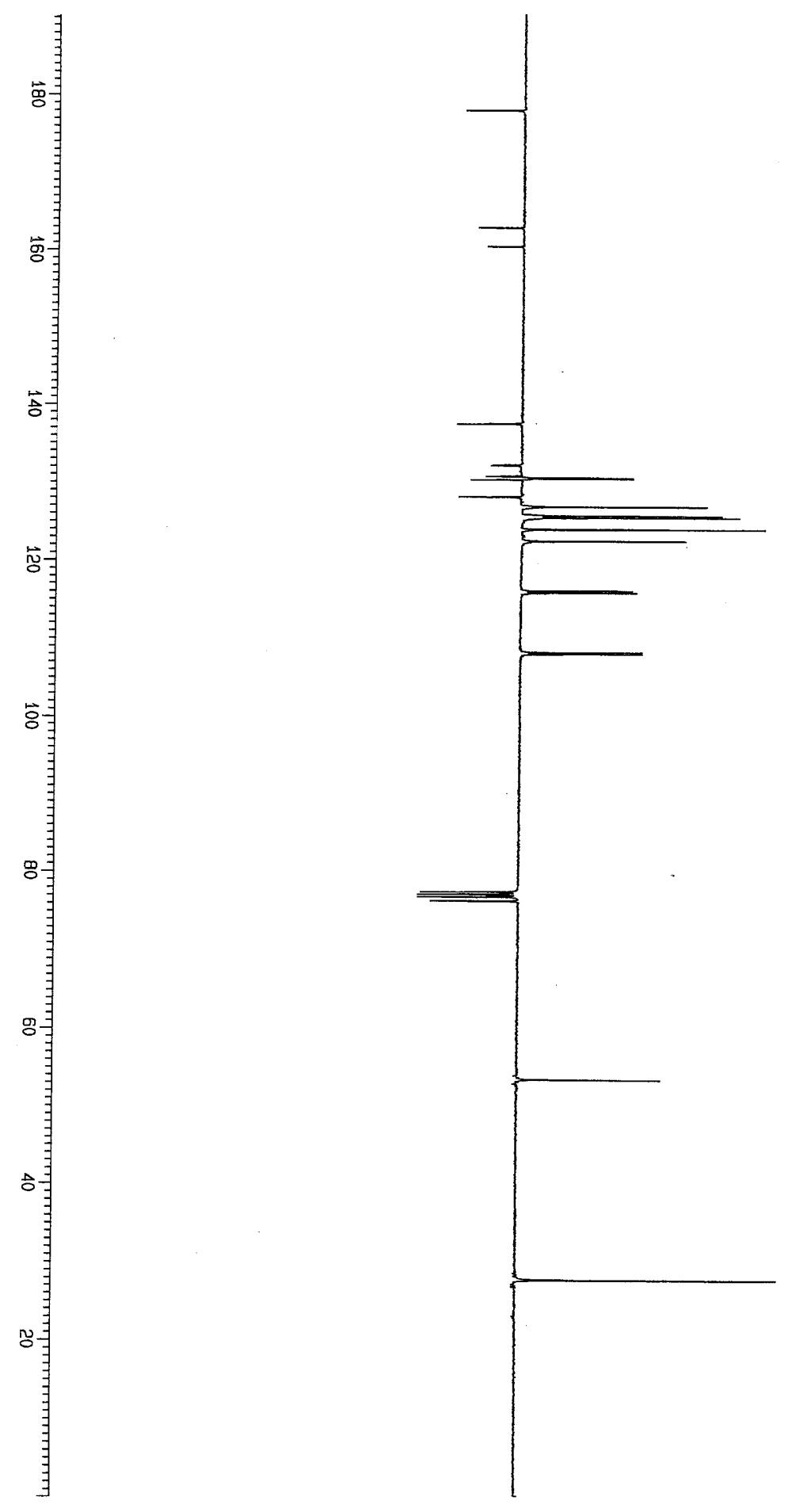

s

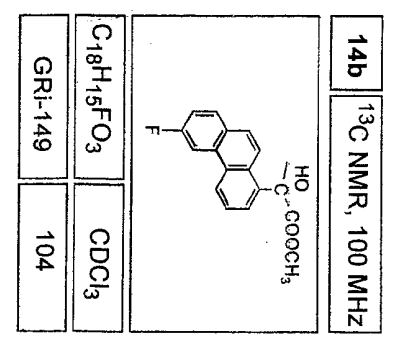




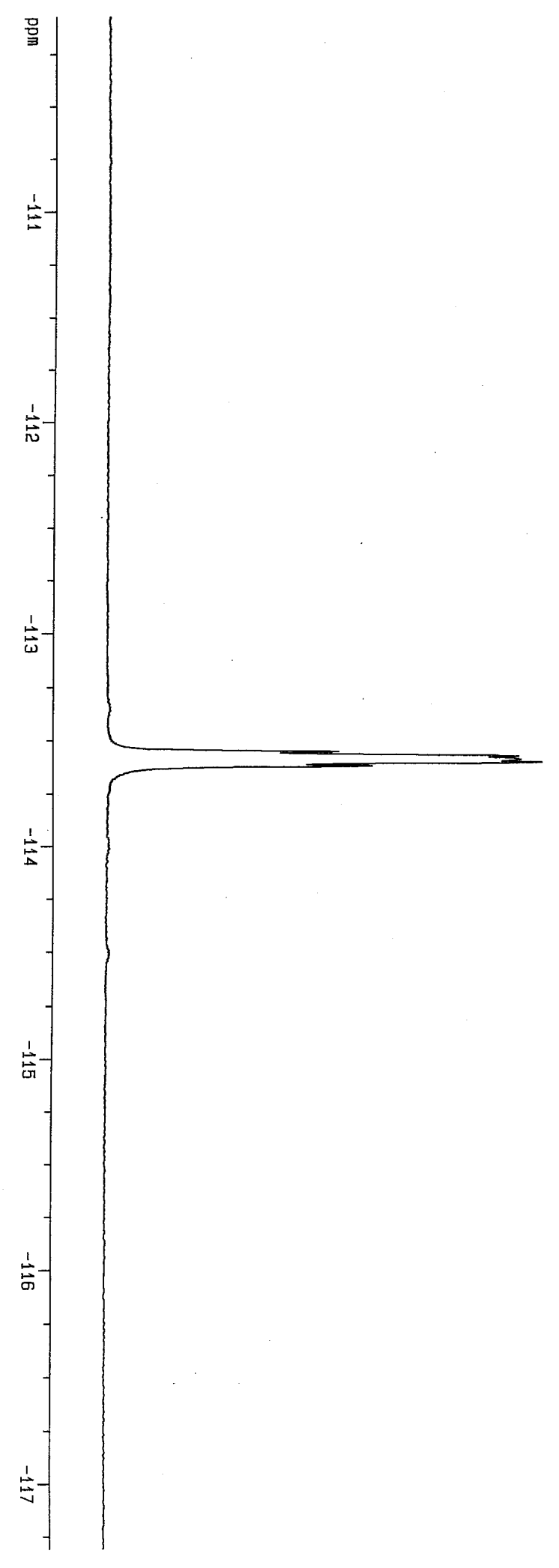

總

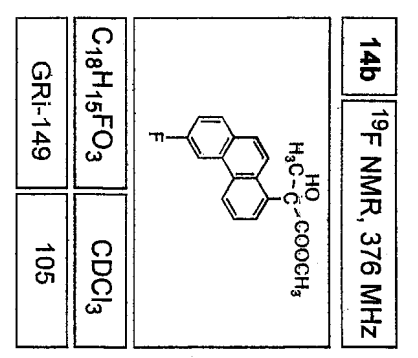




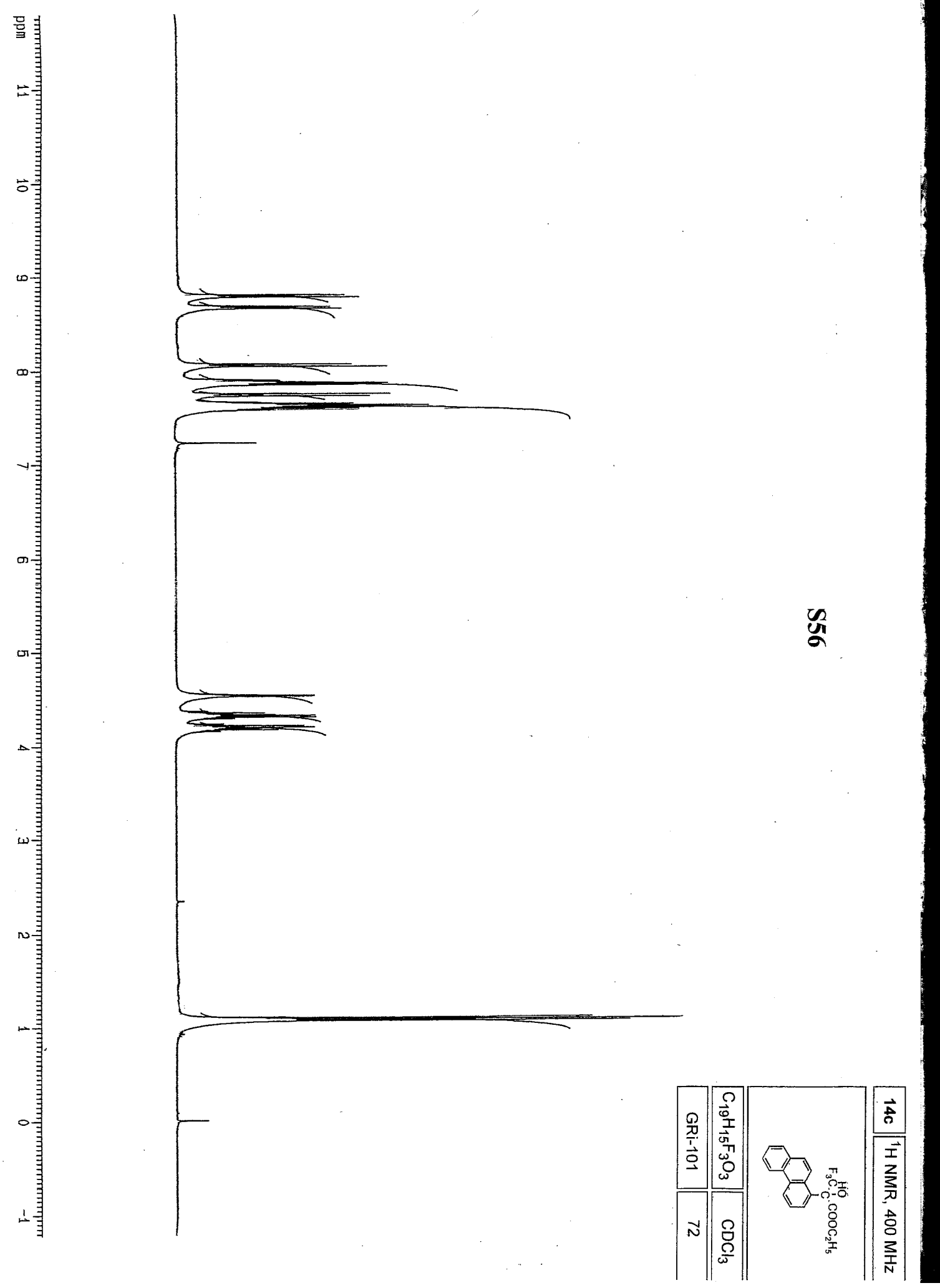



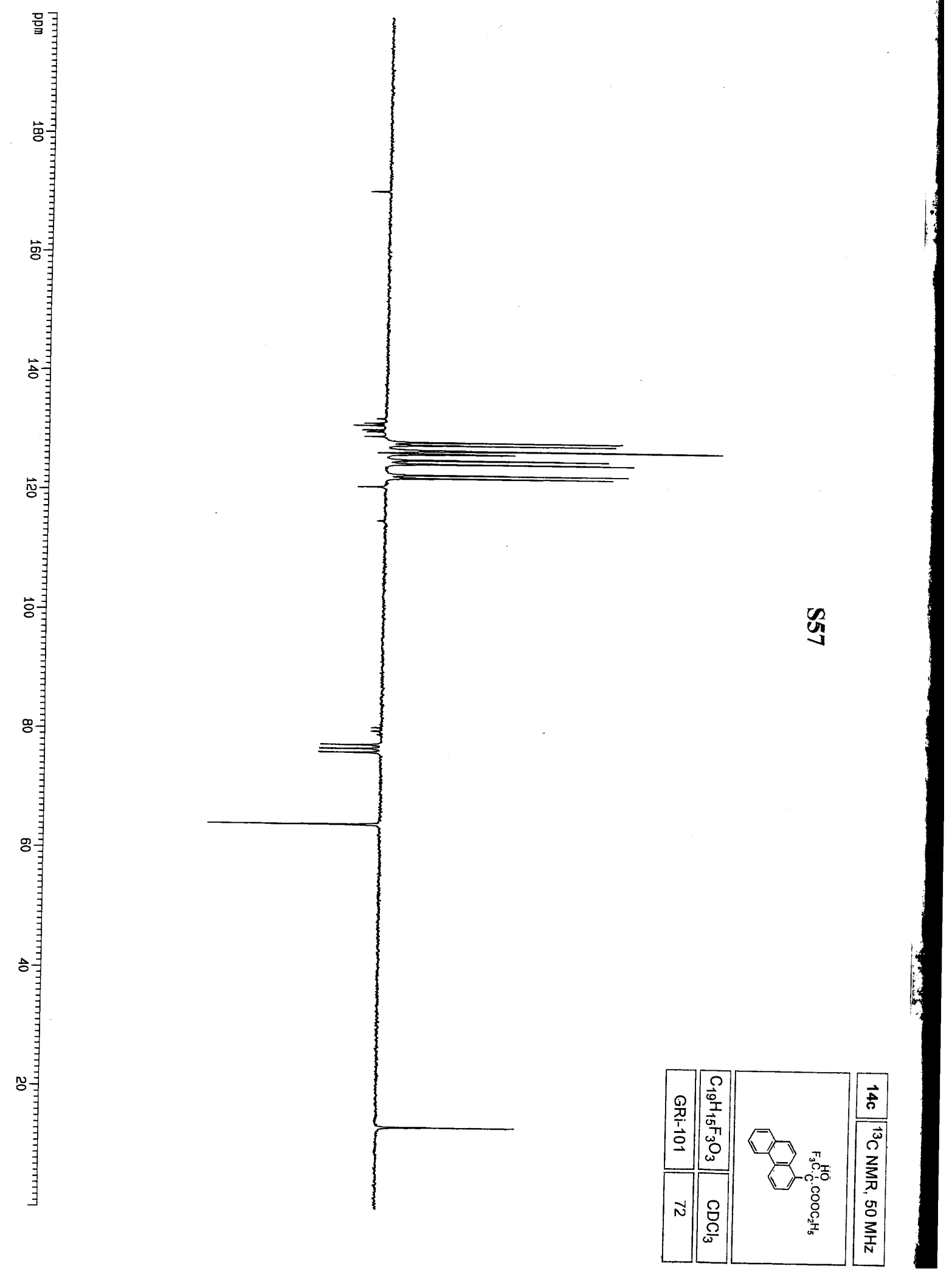


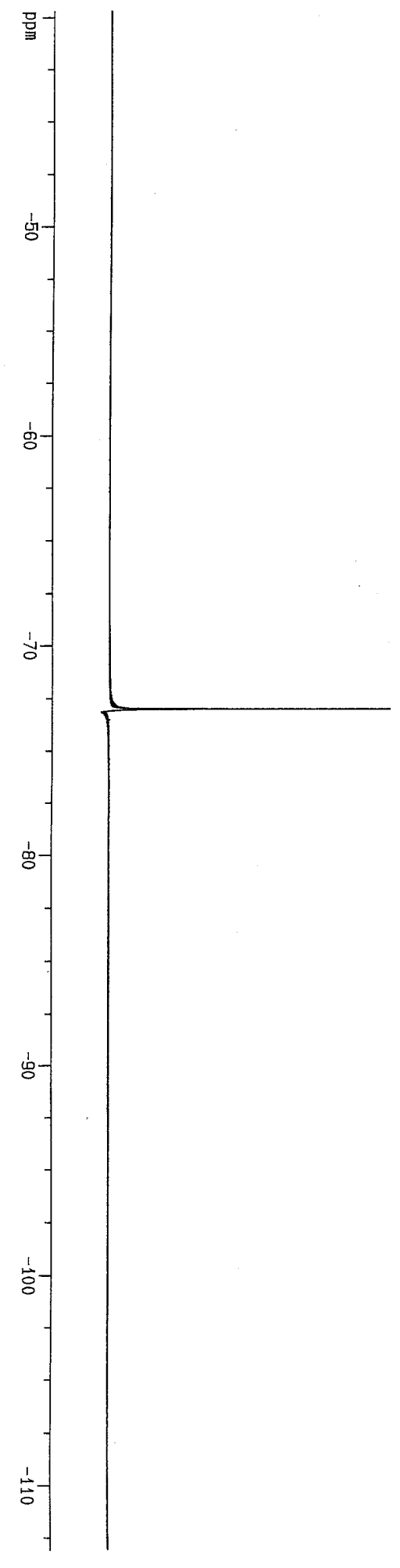

说

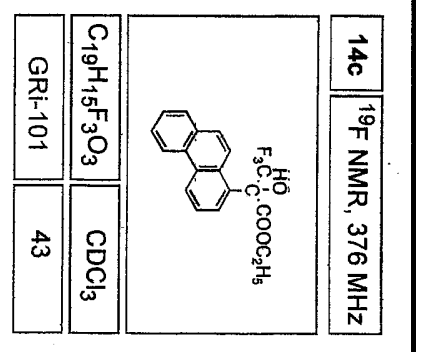



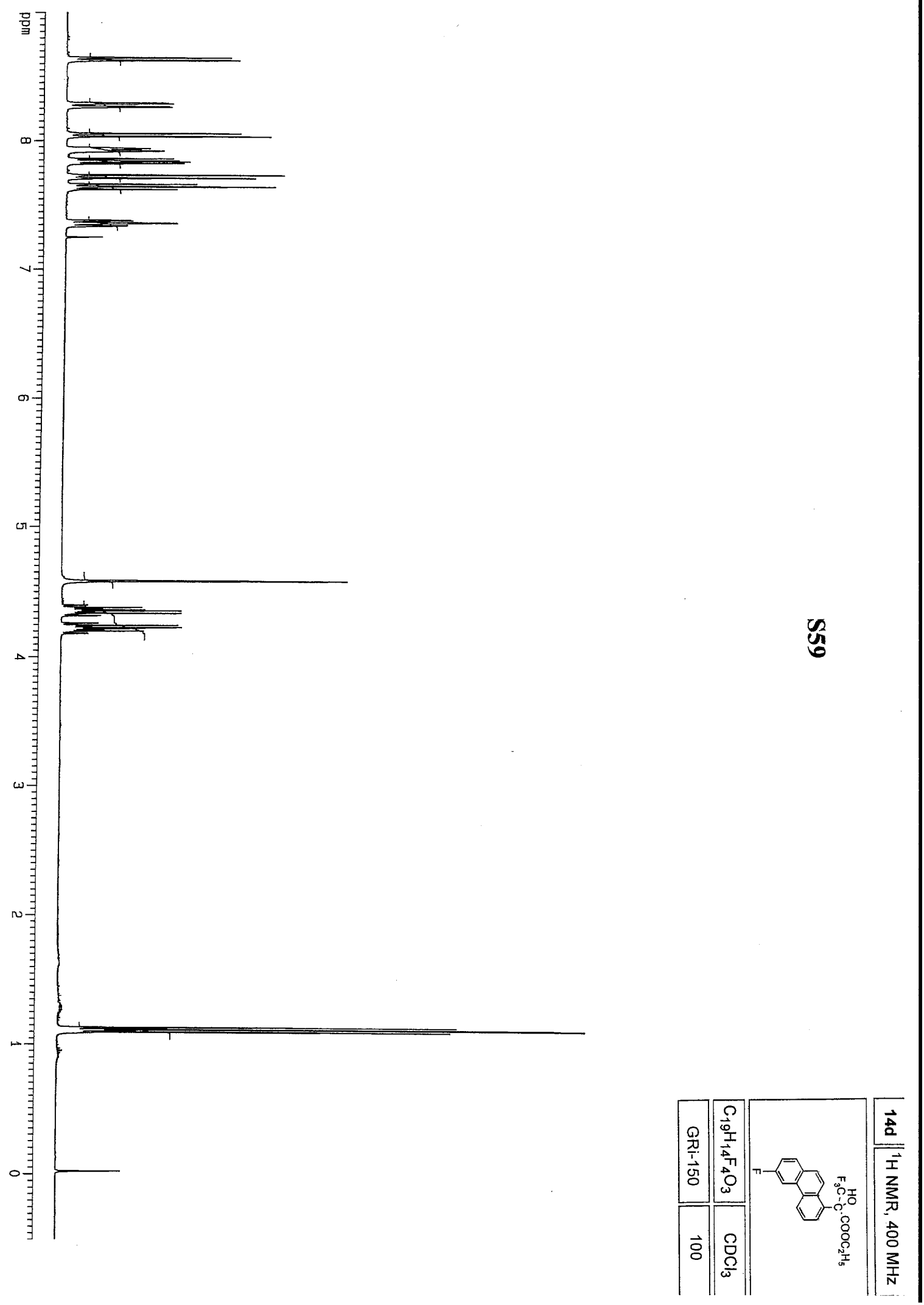

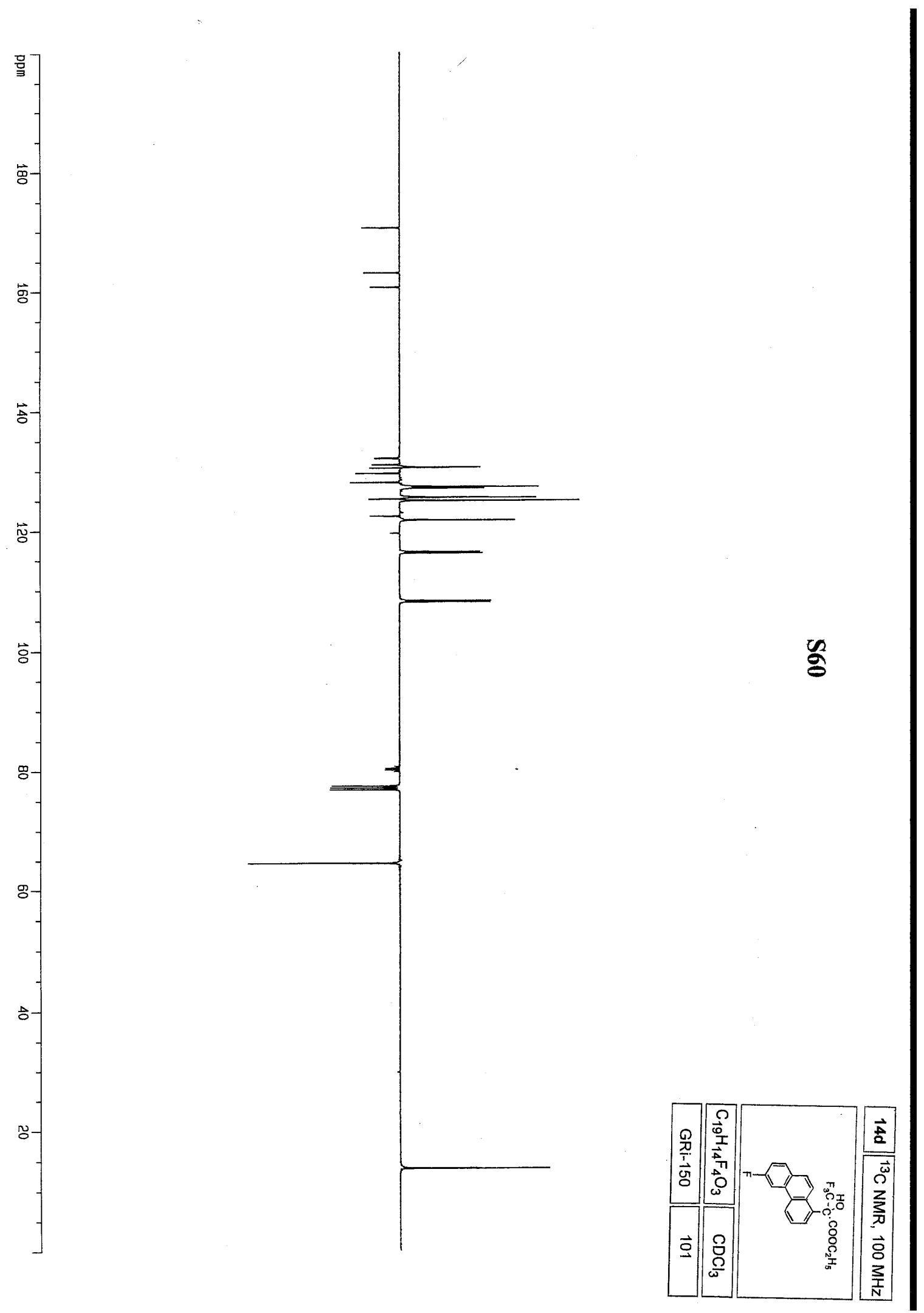

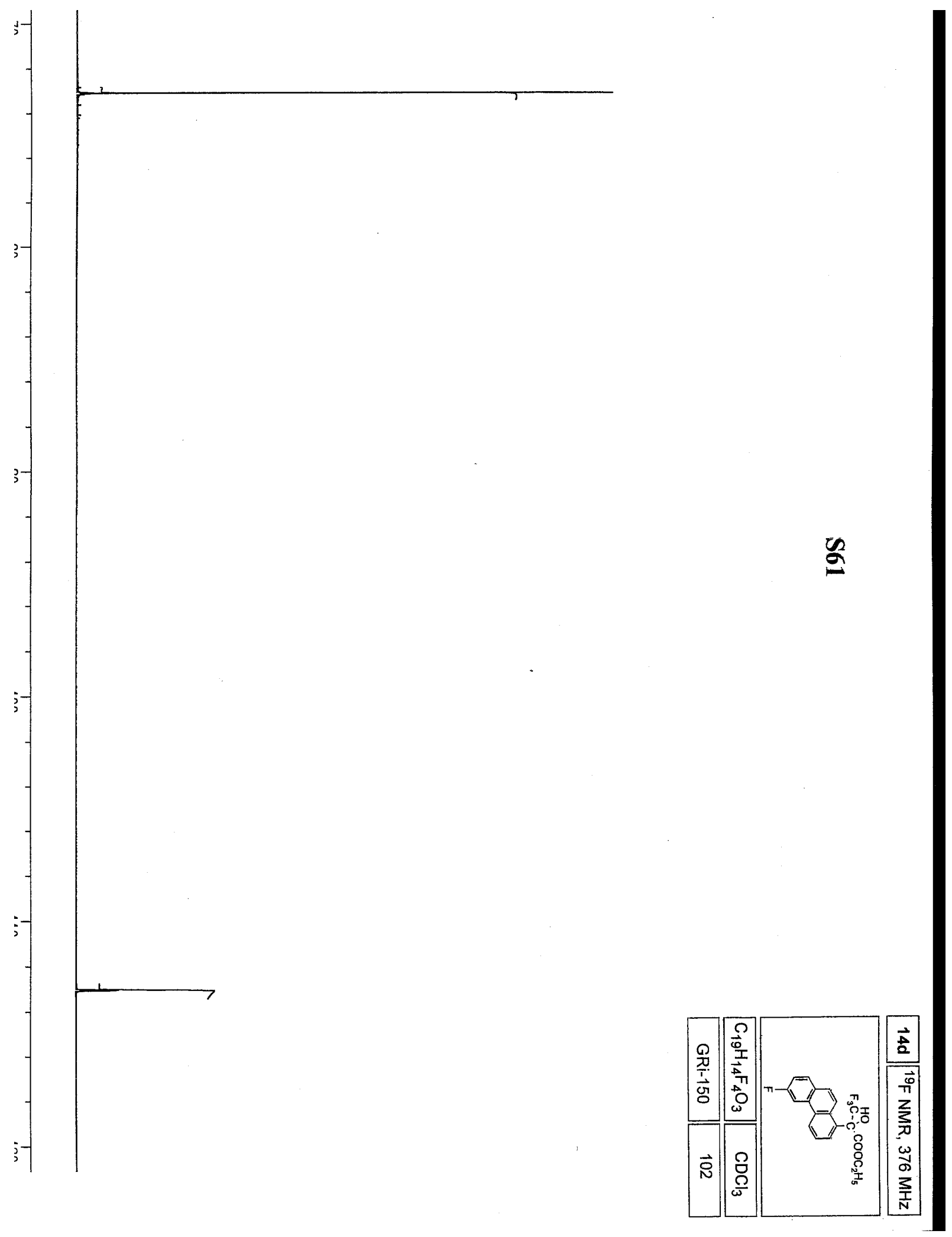

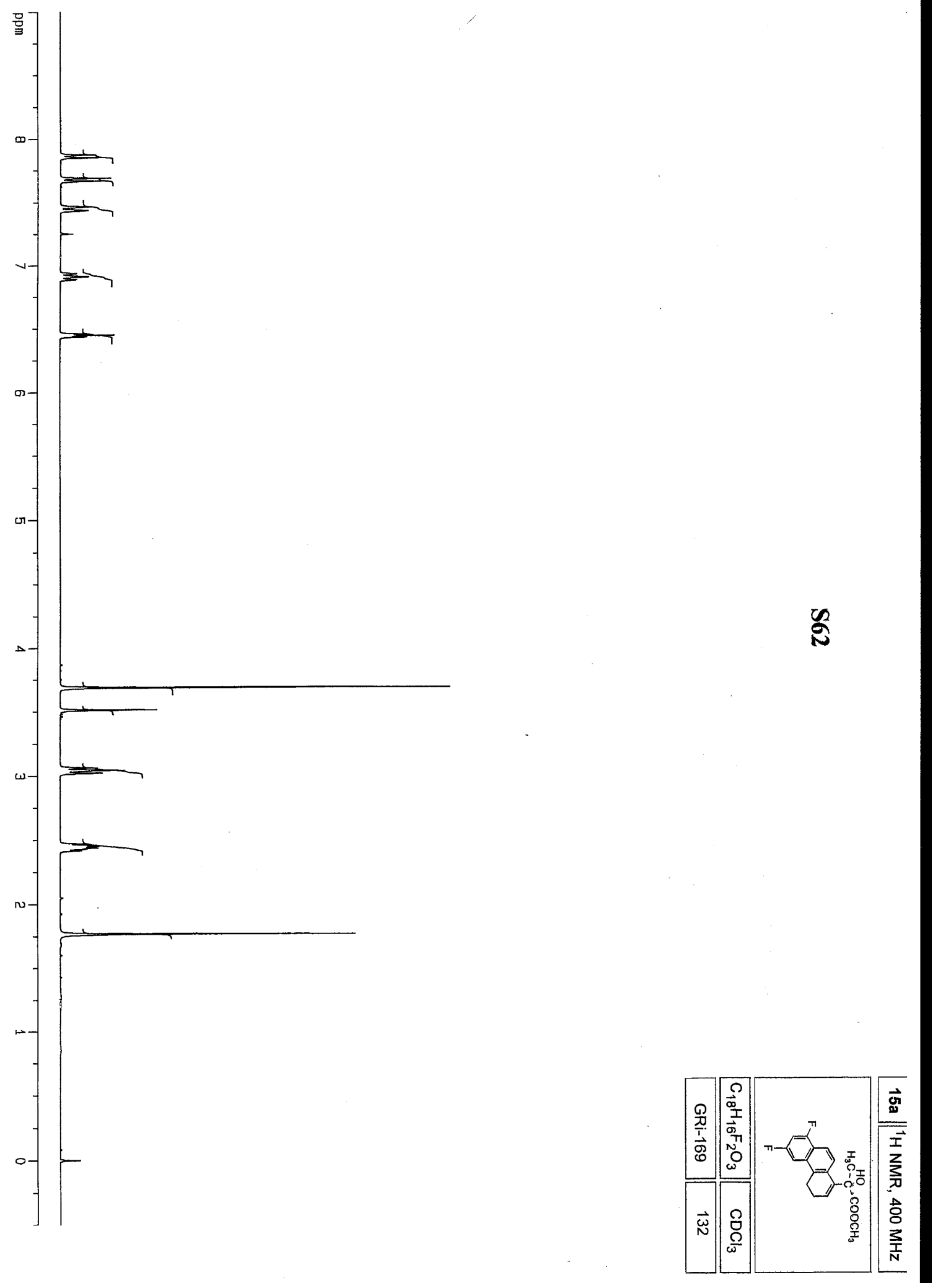

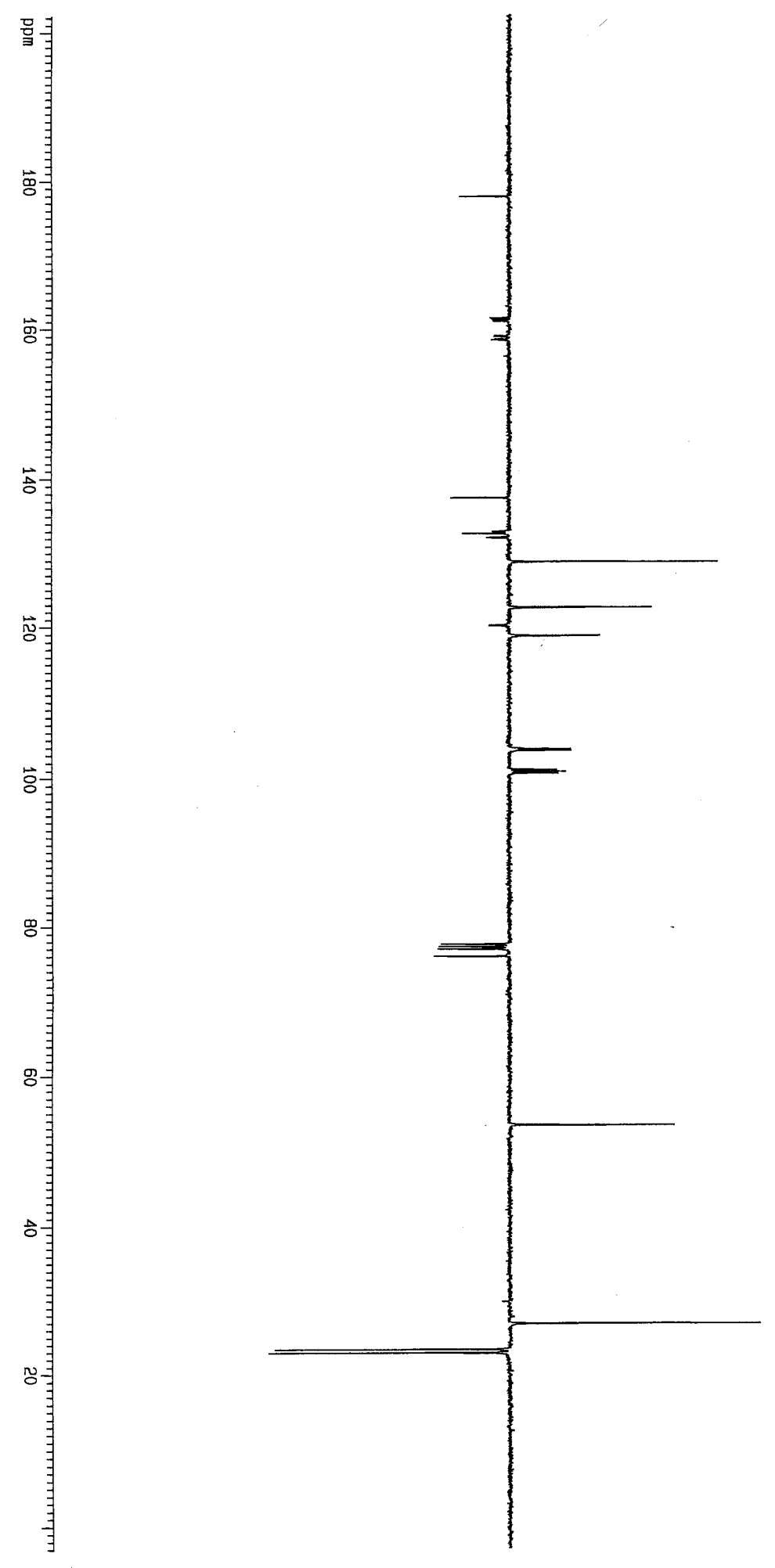


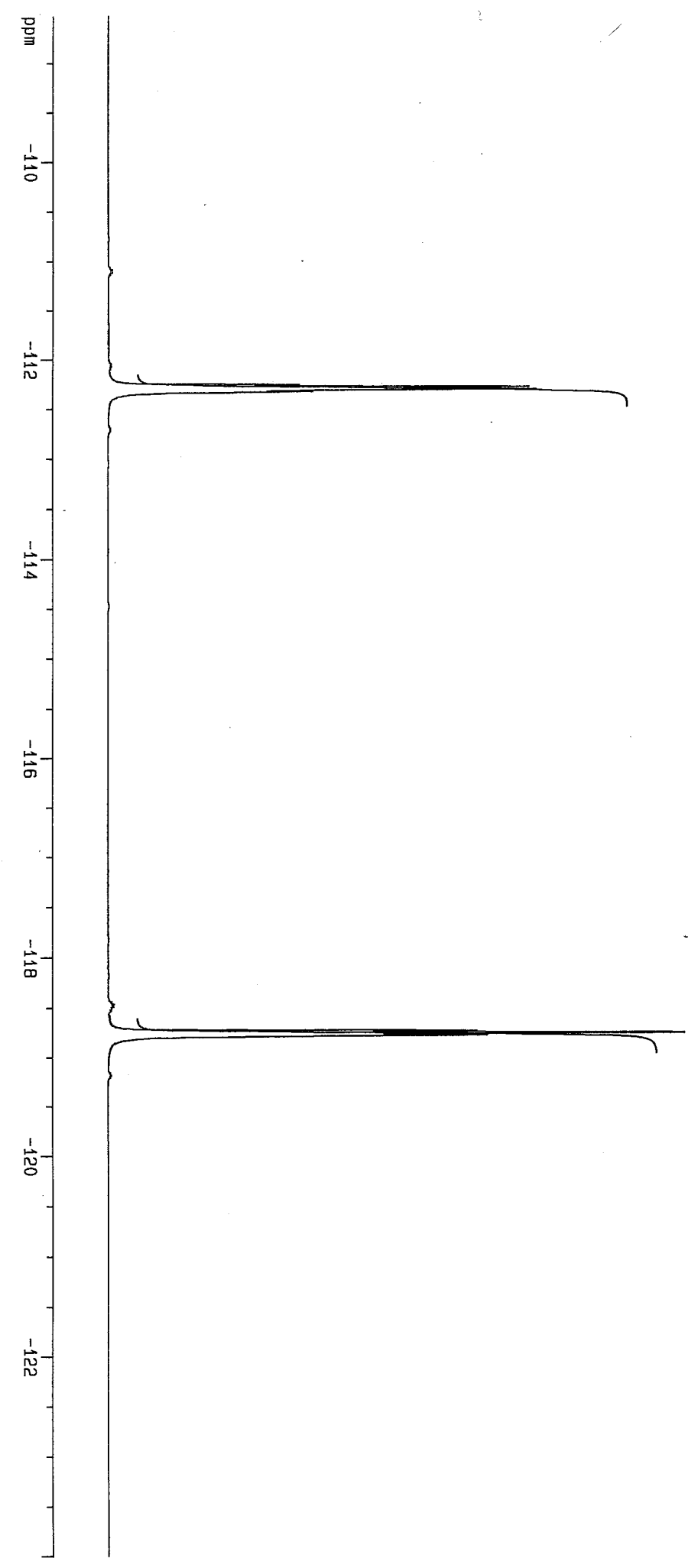

经

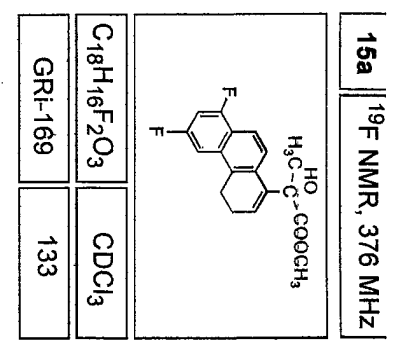



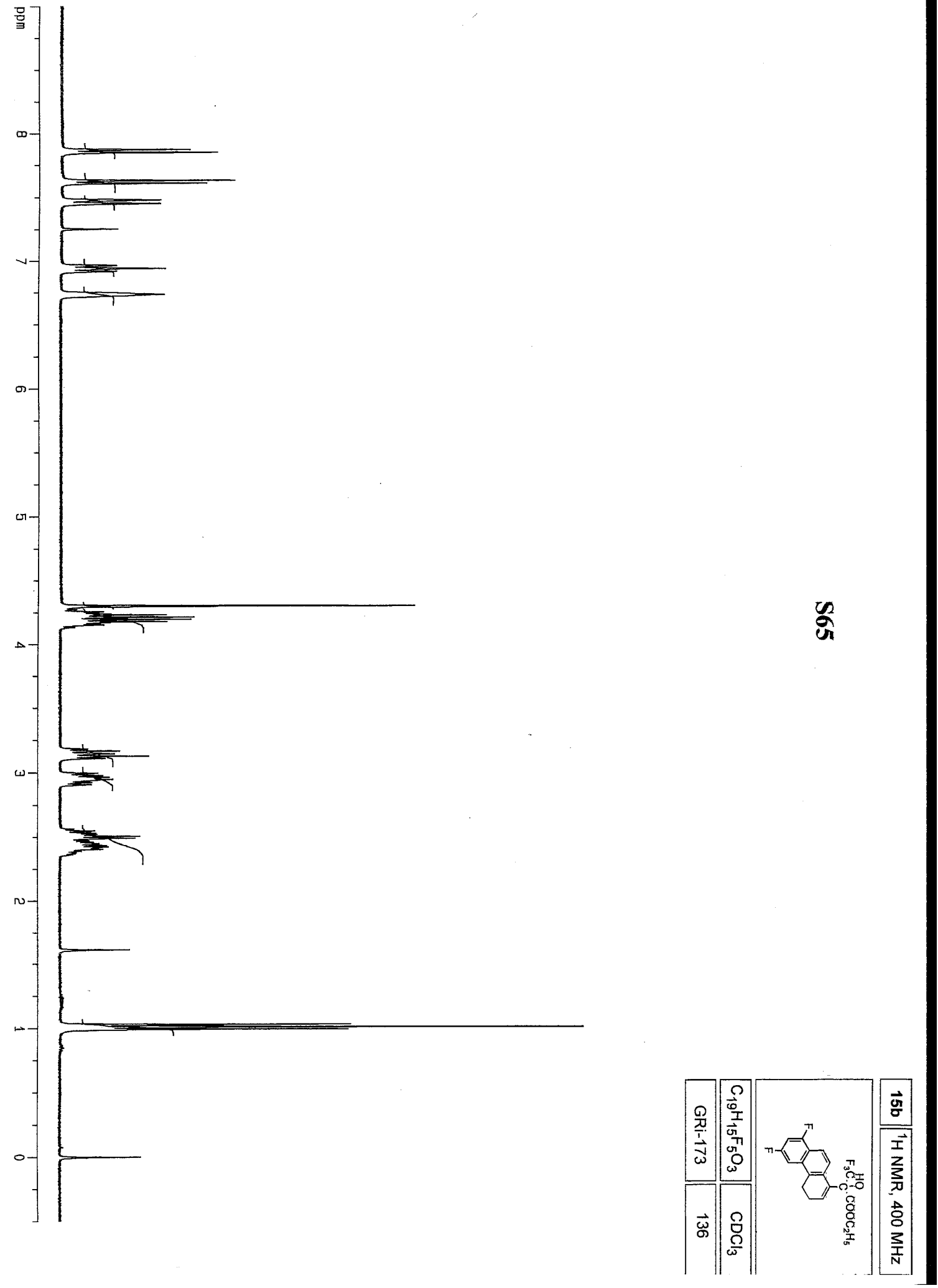

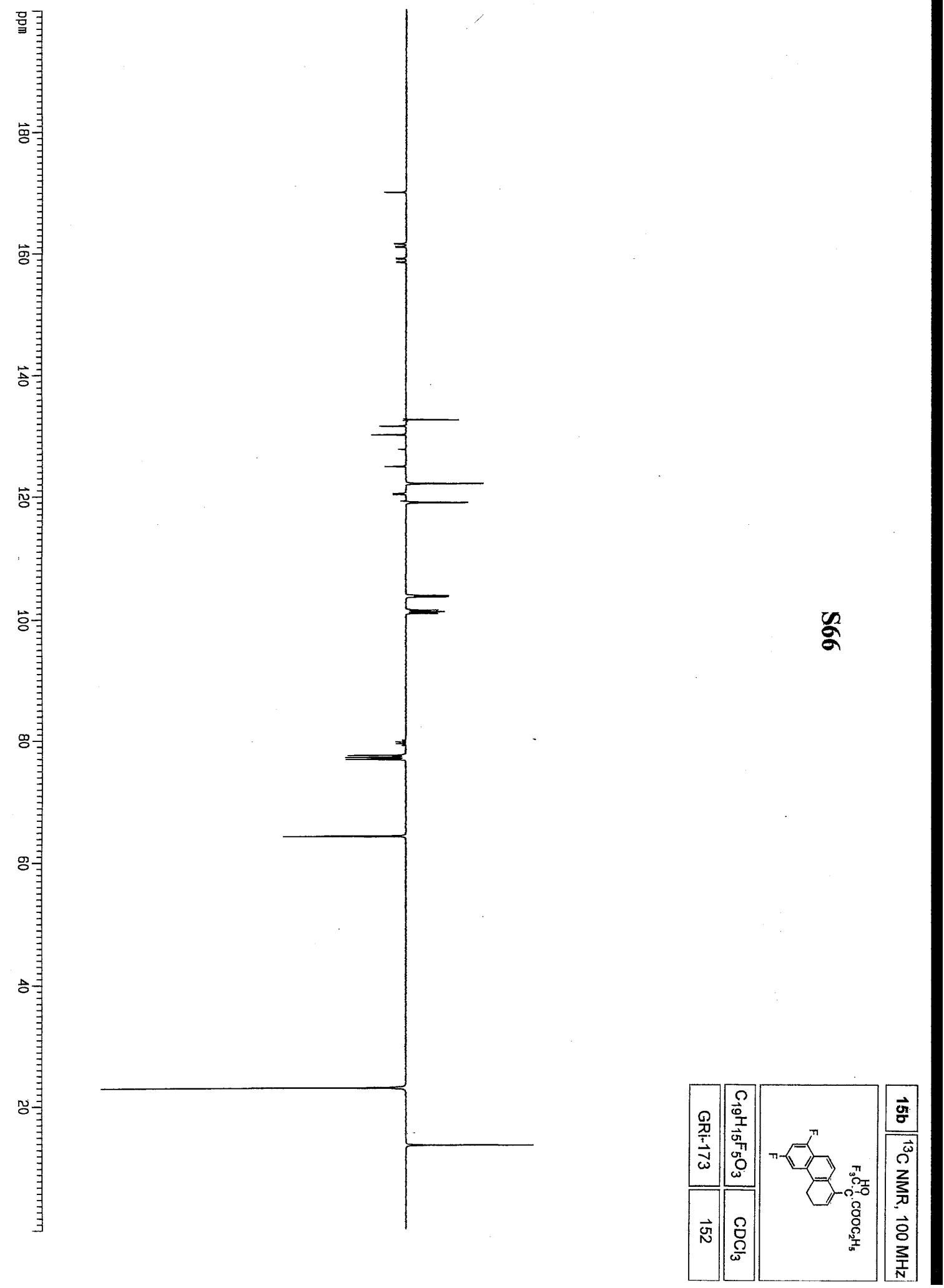

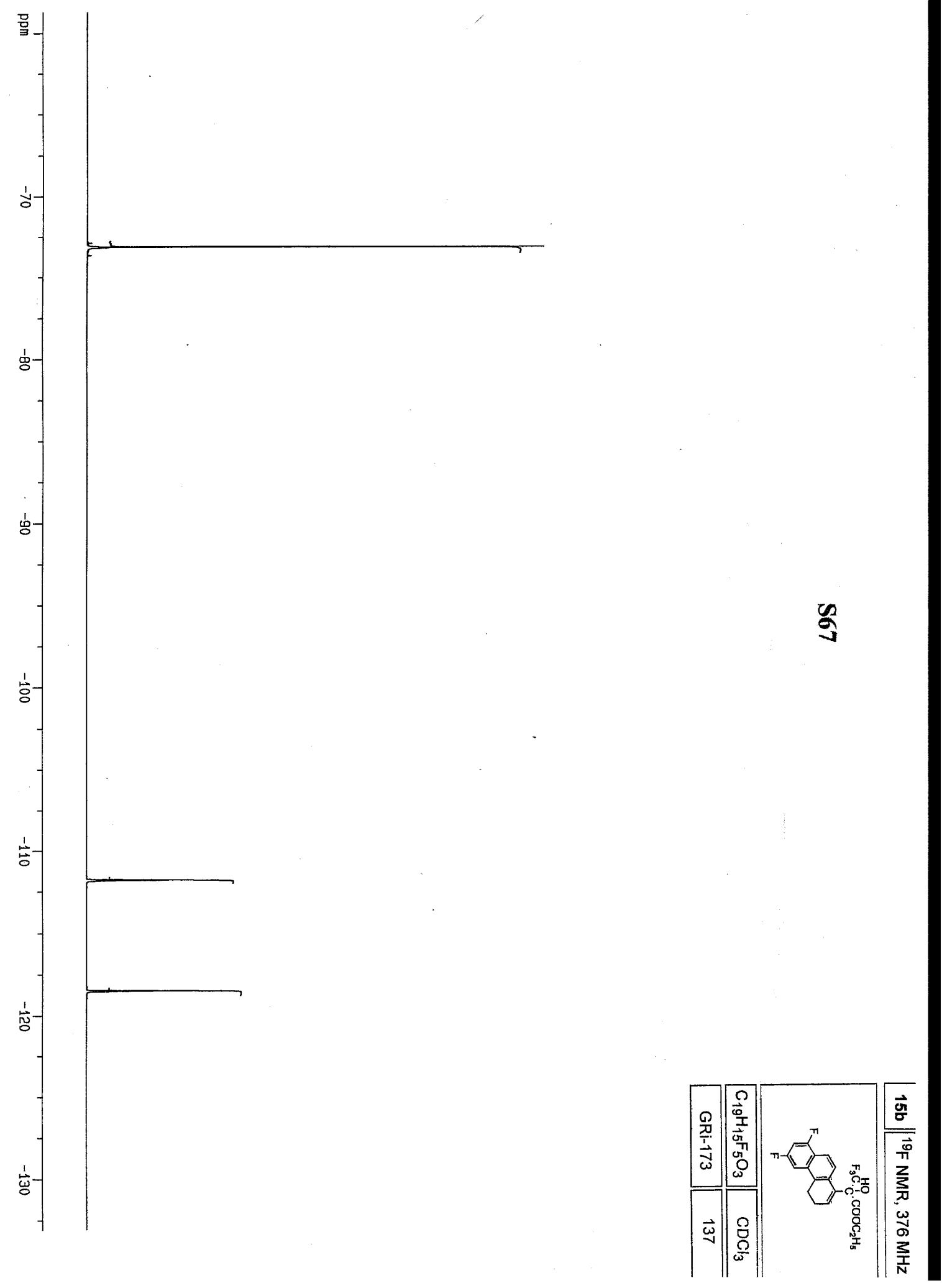


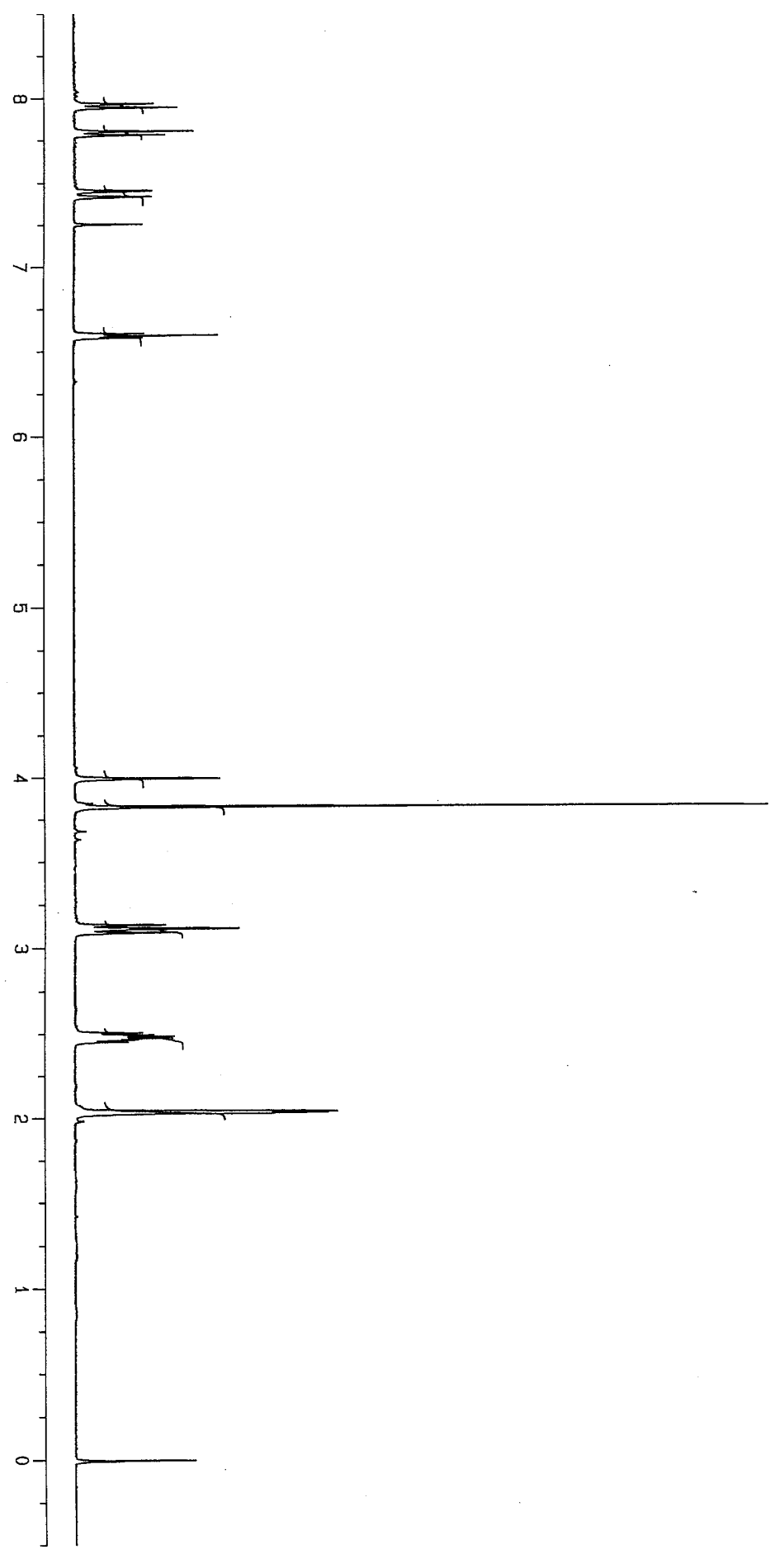

$\mathscr{\infty}$

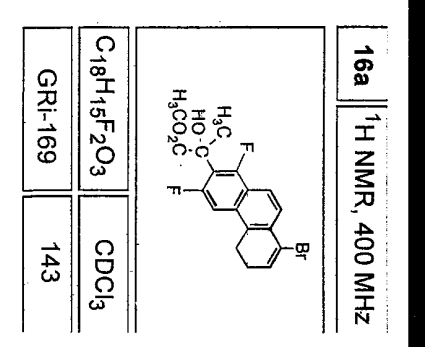



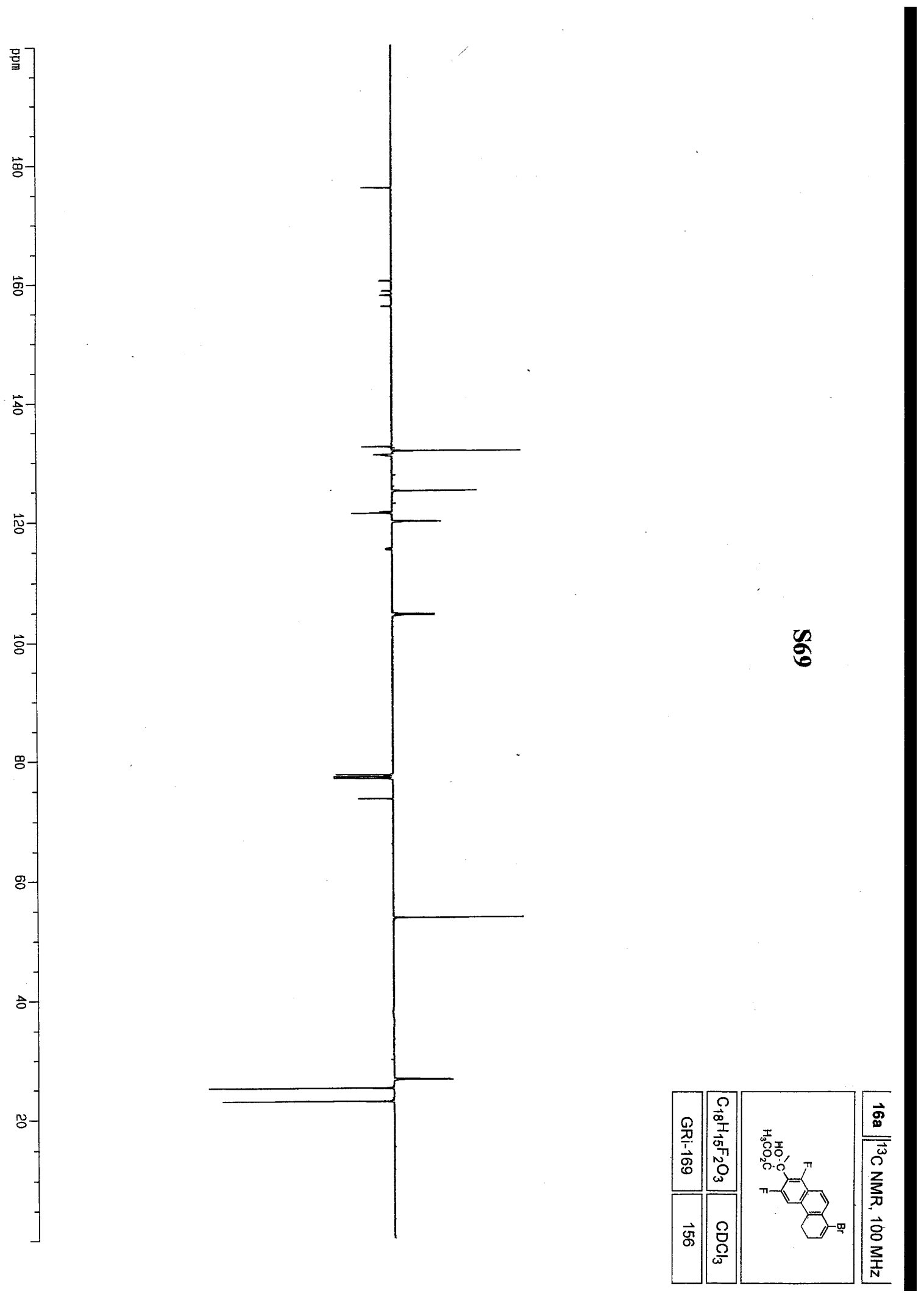

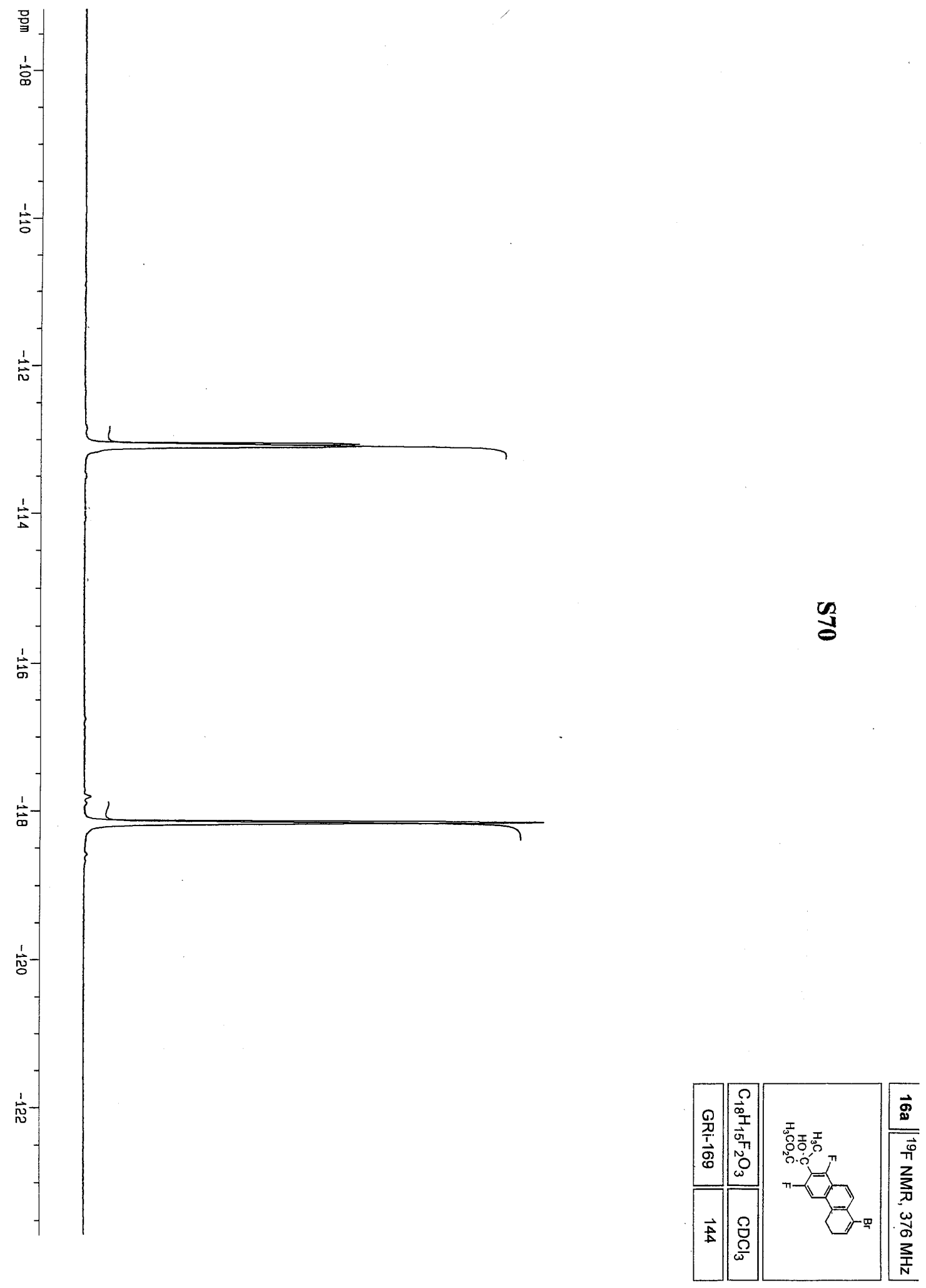

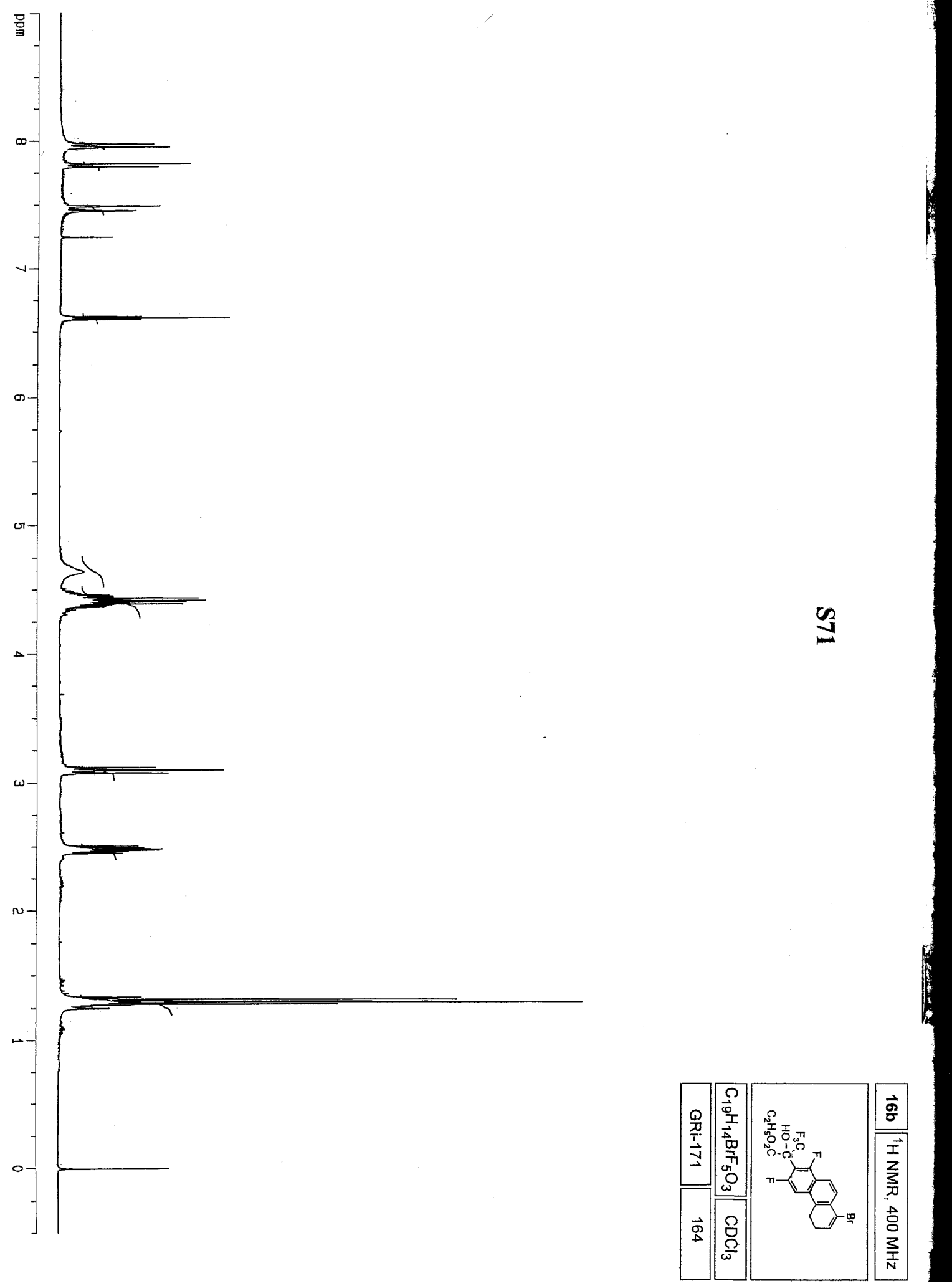

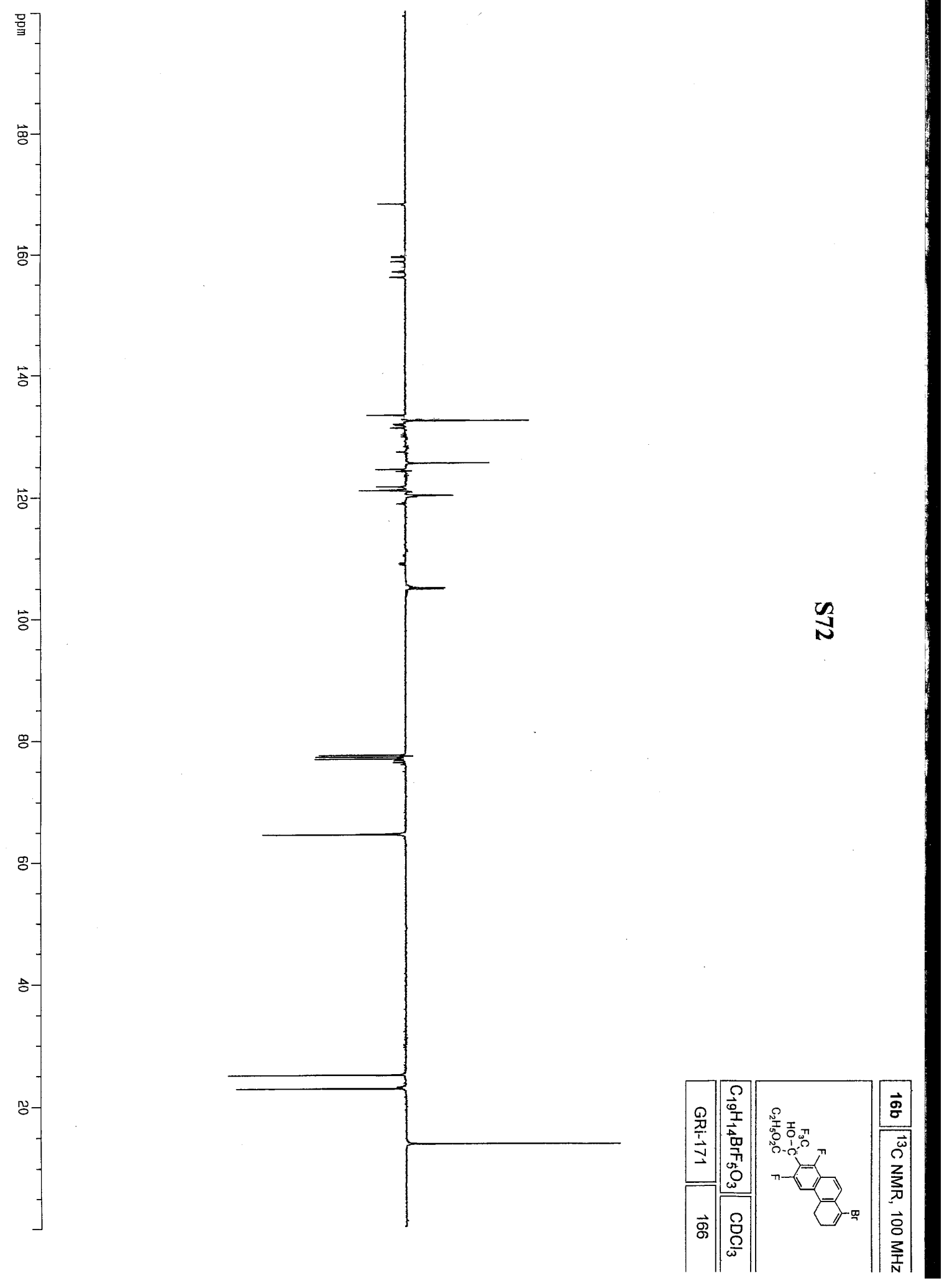


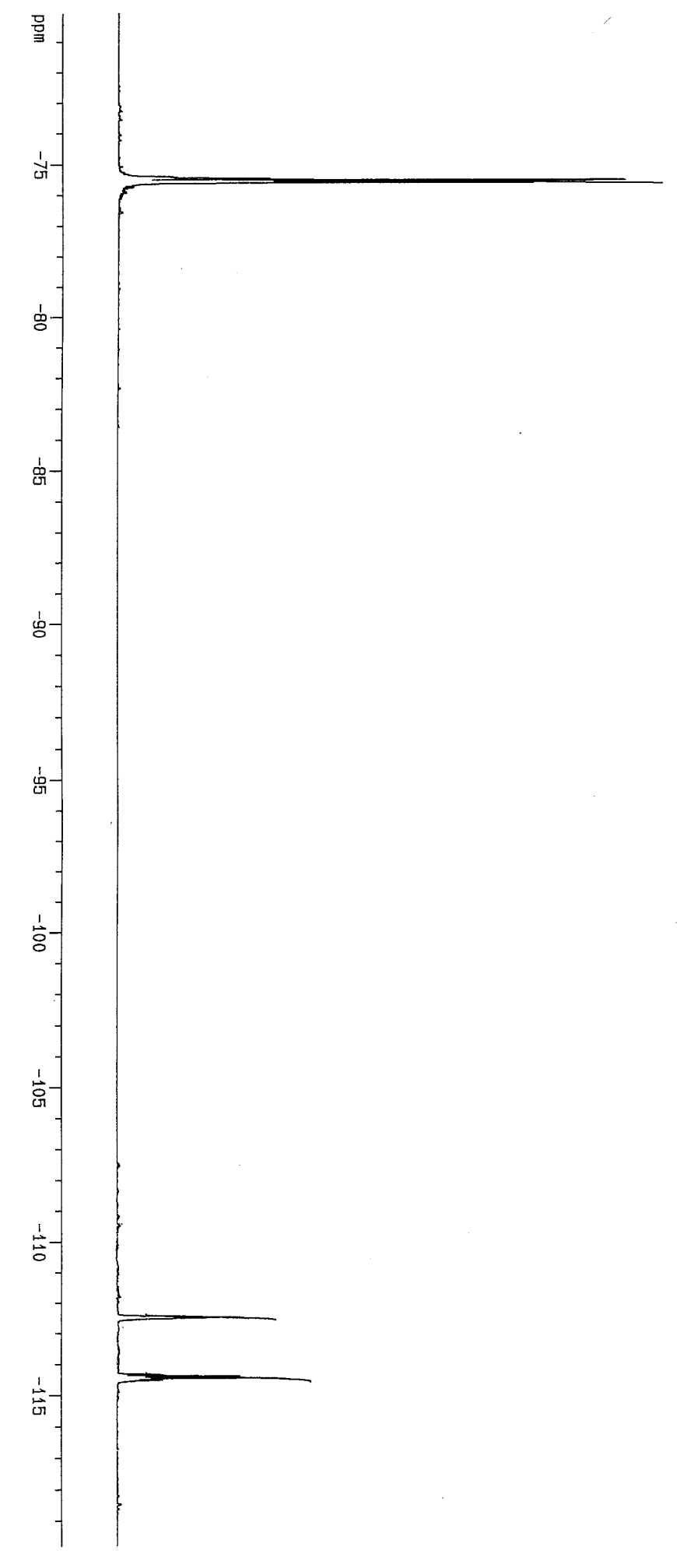

5

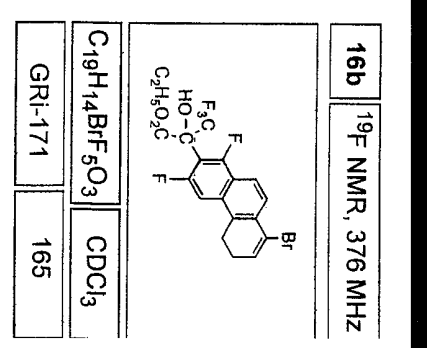



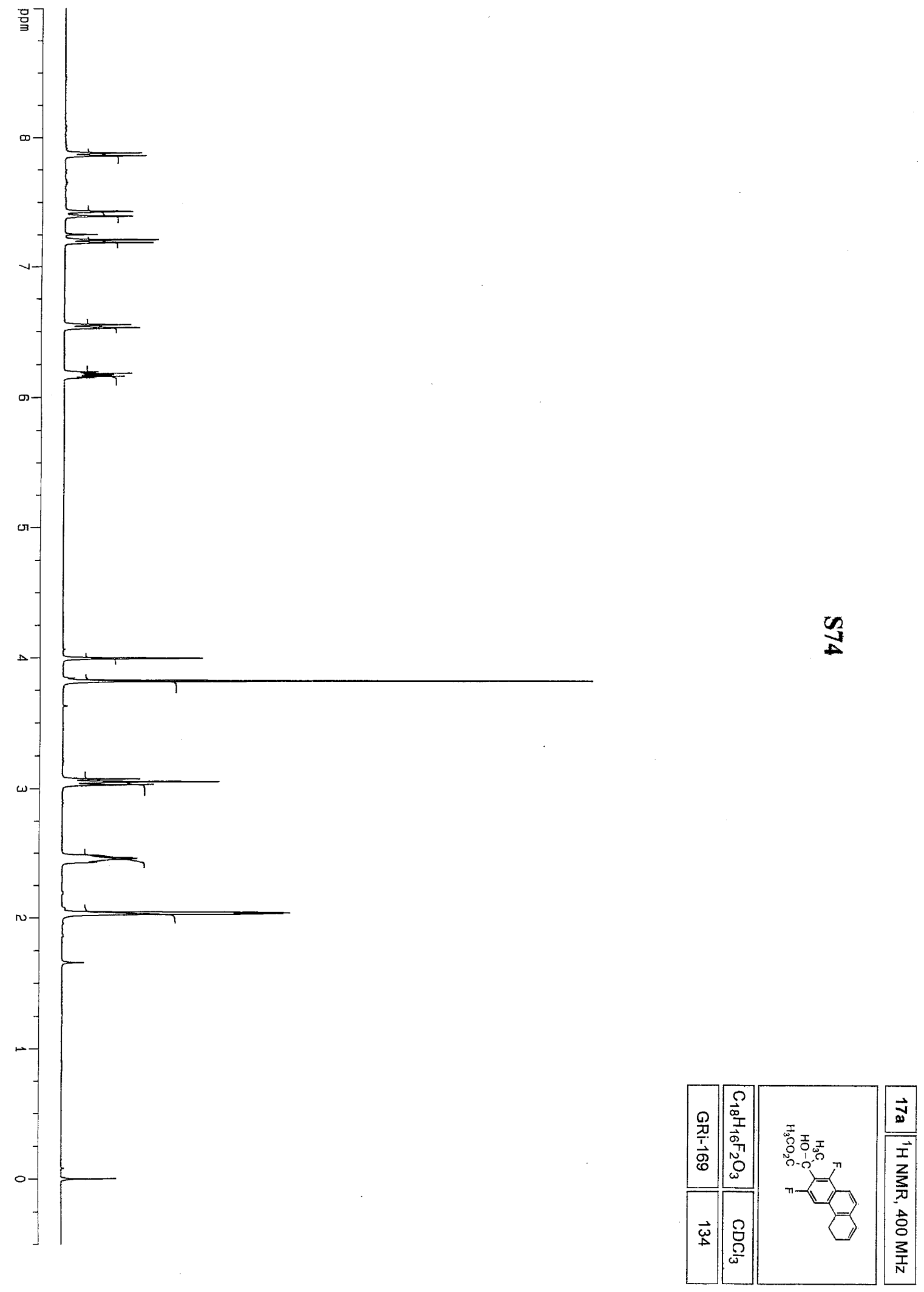

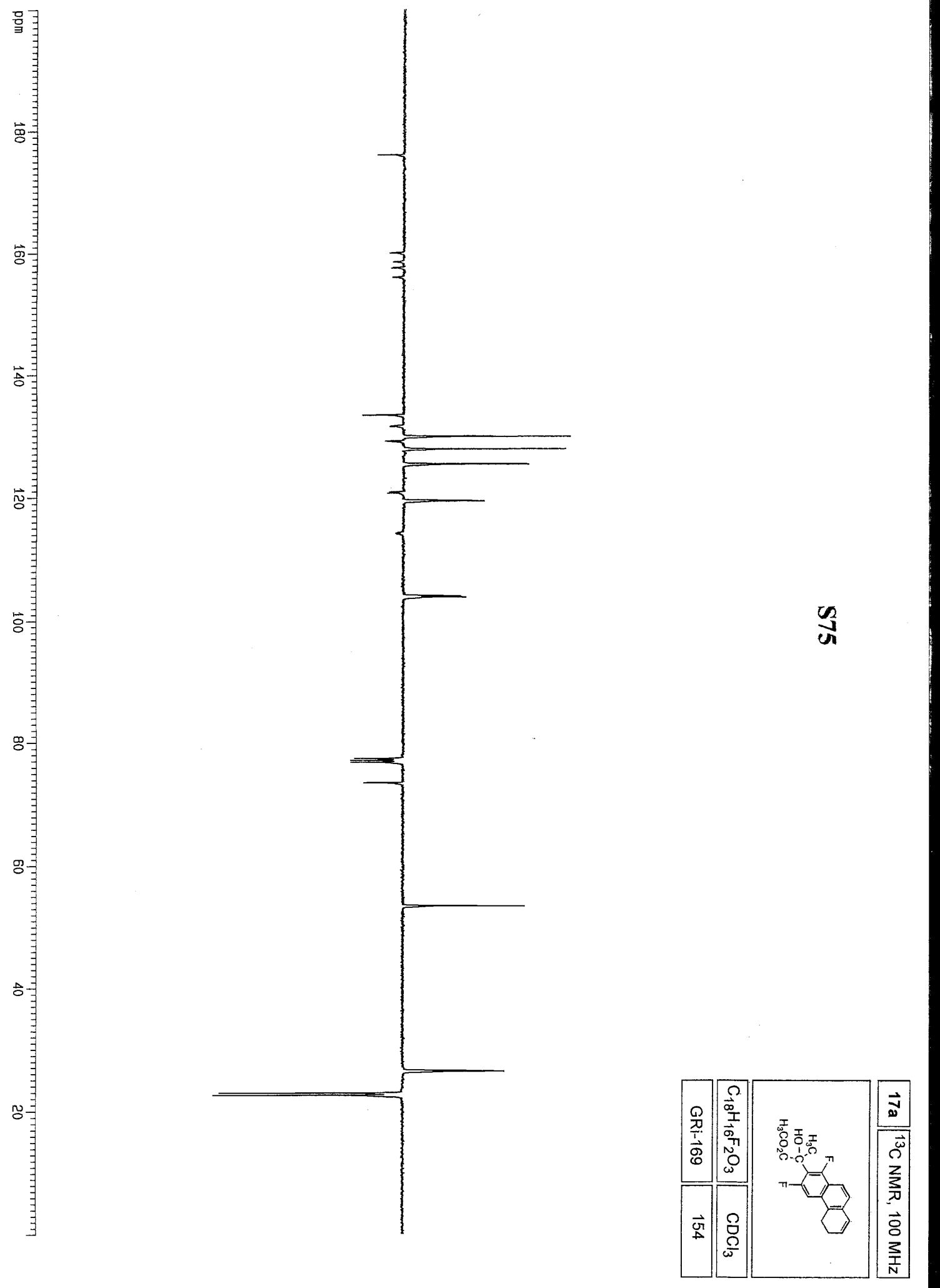

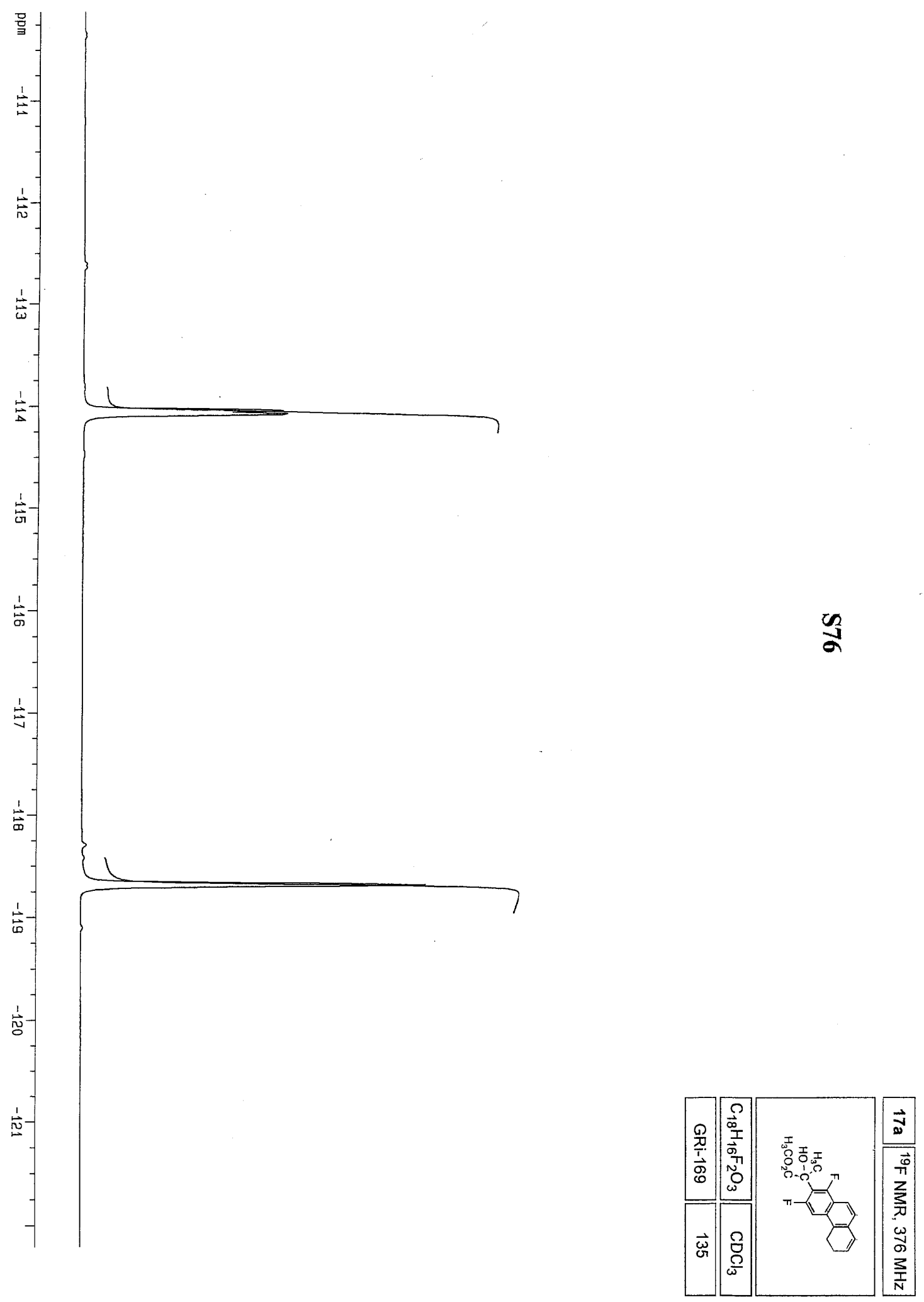


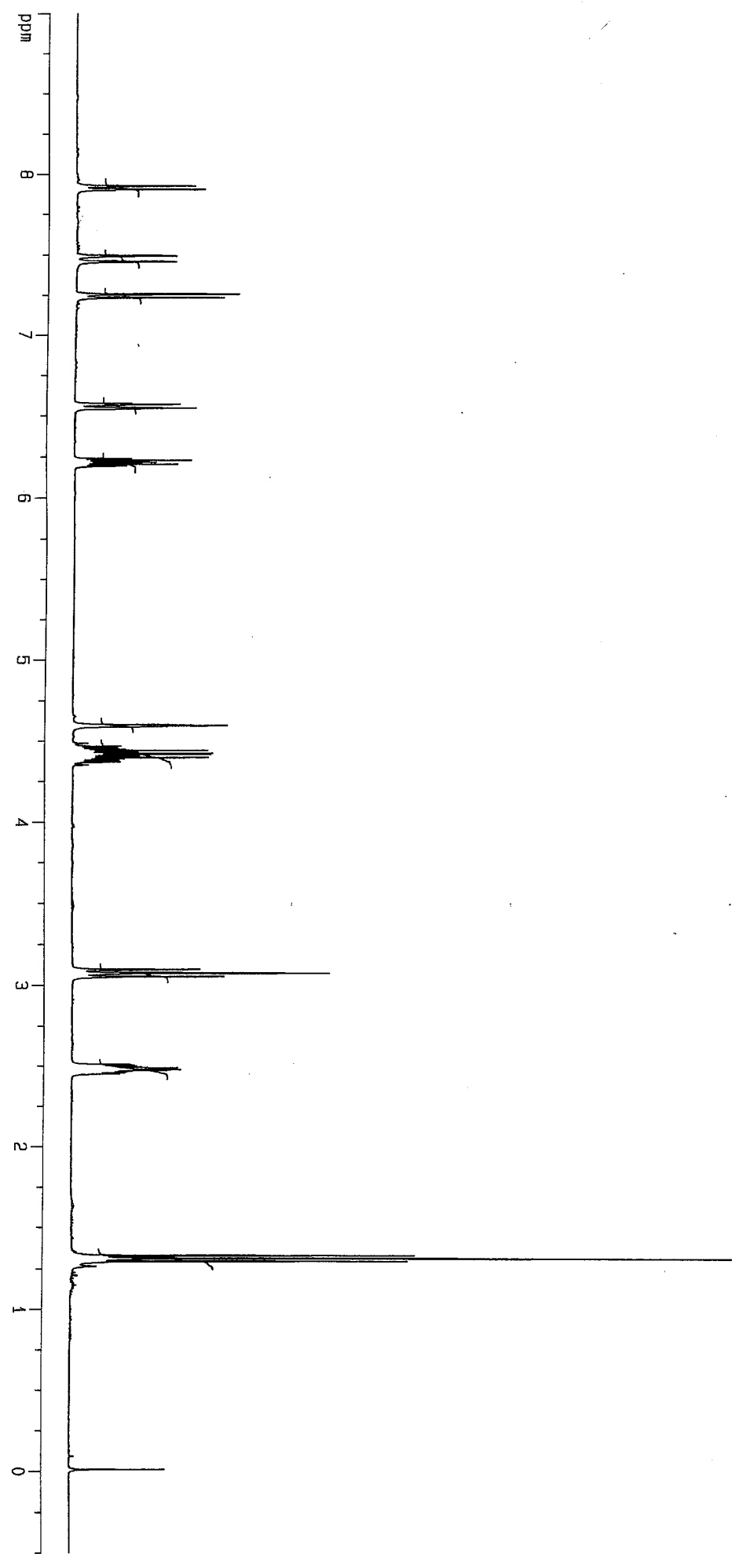

s

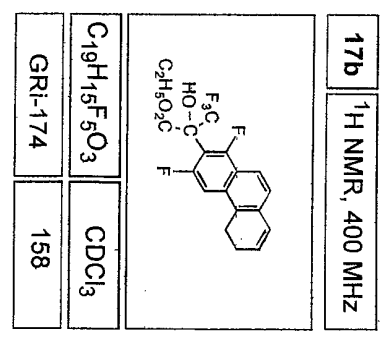



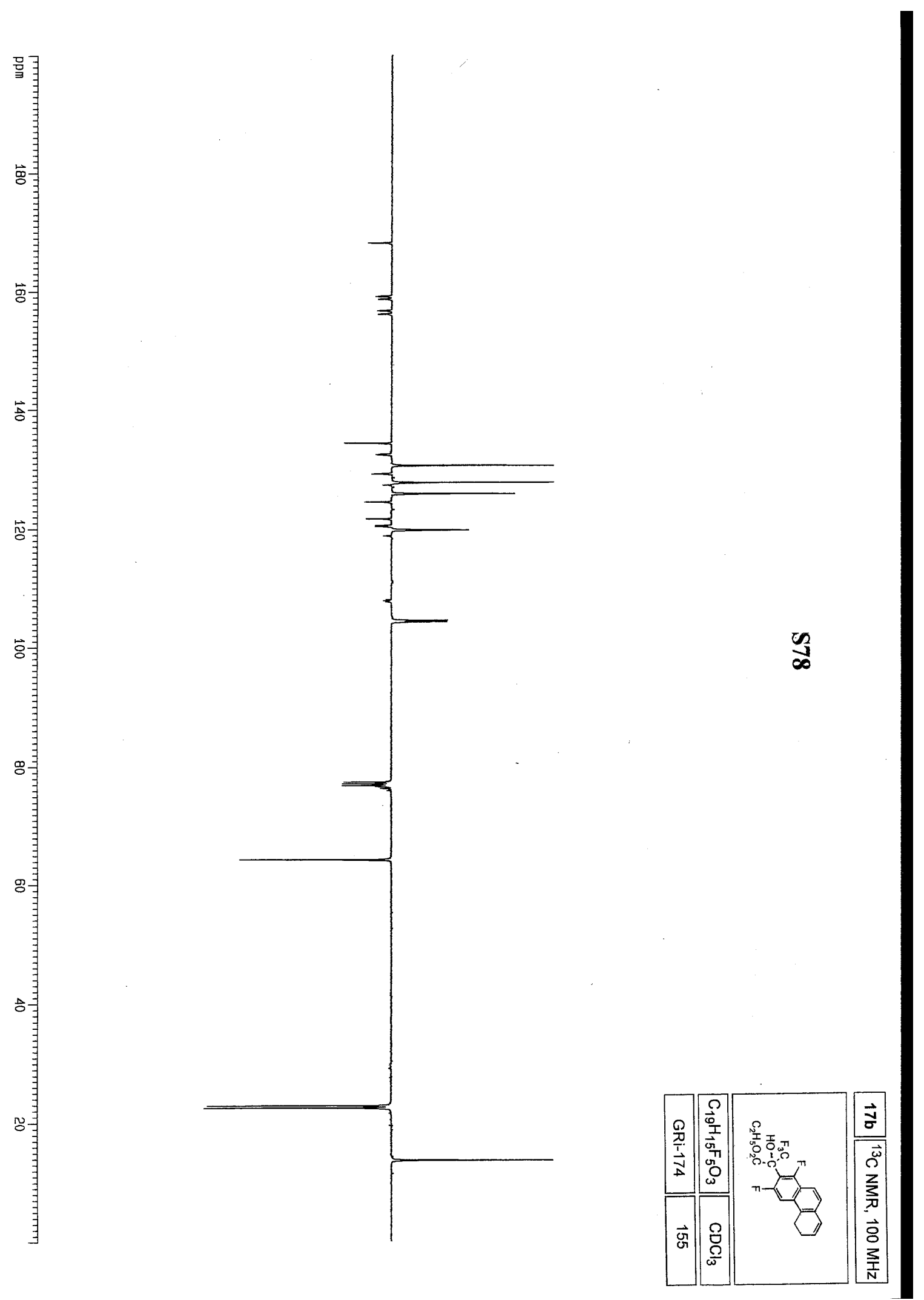


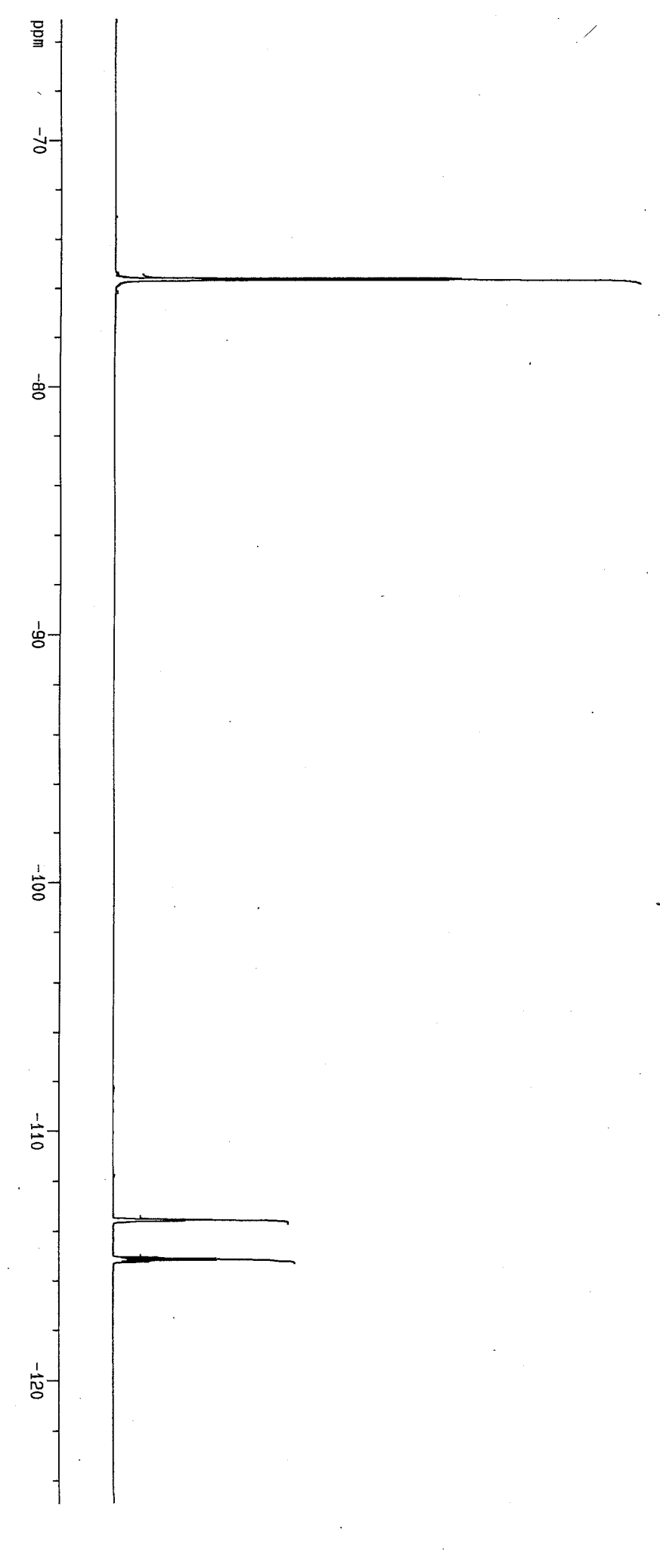

ป

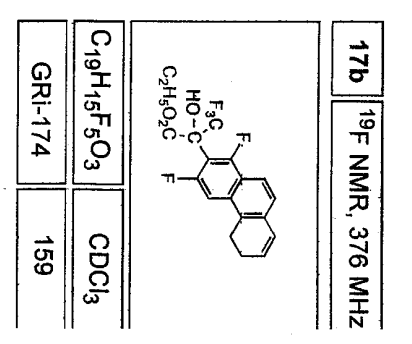




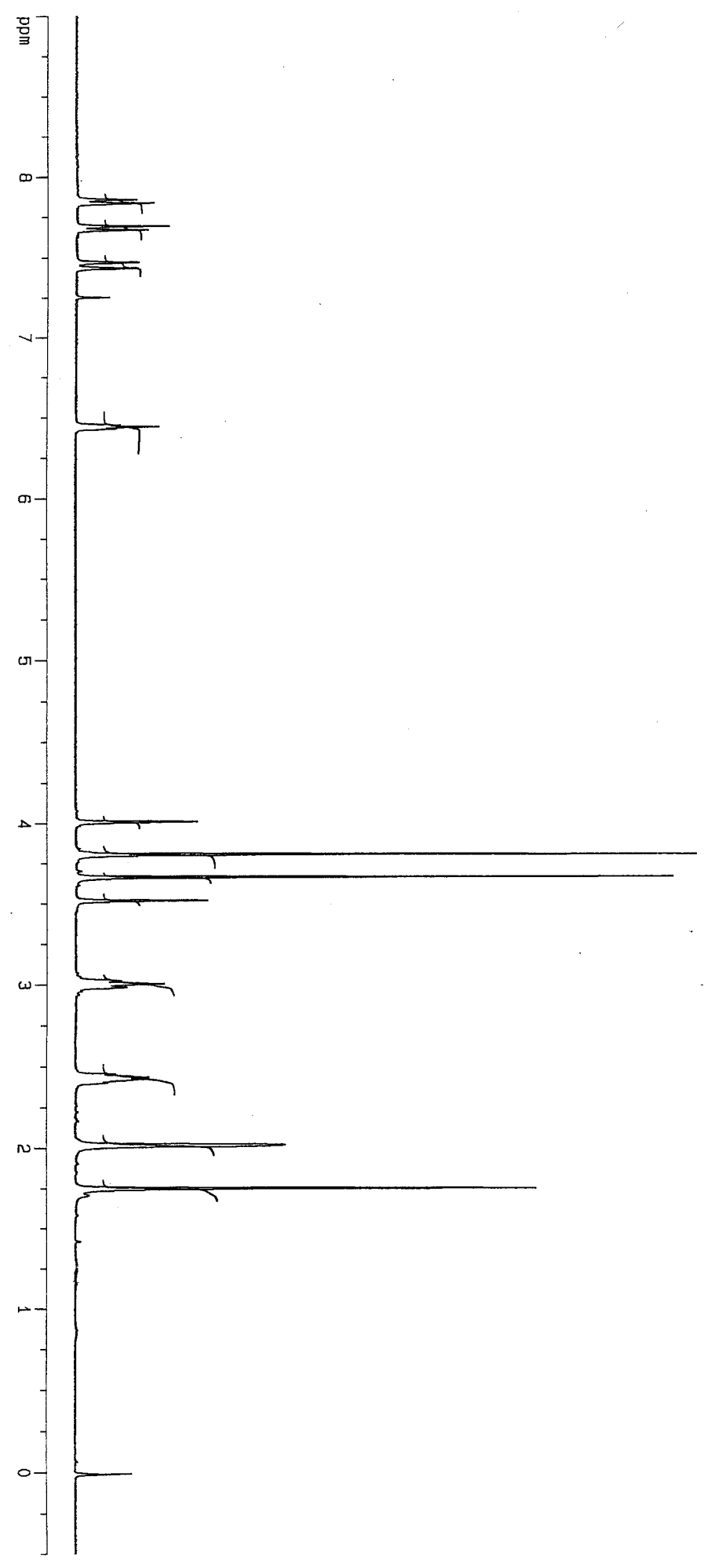



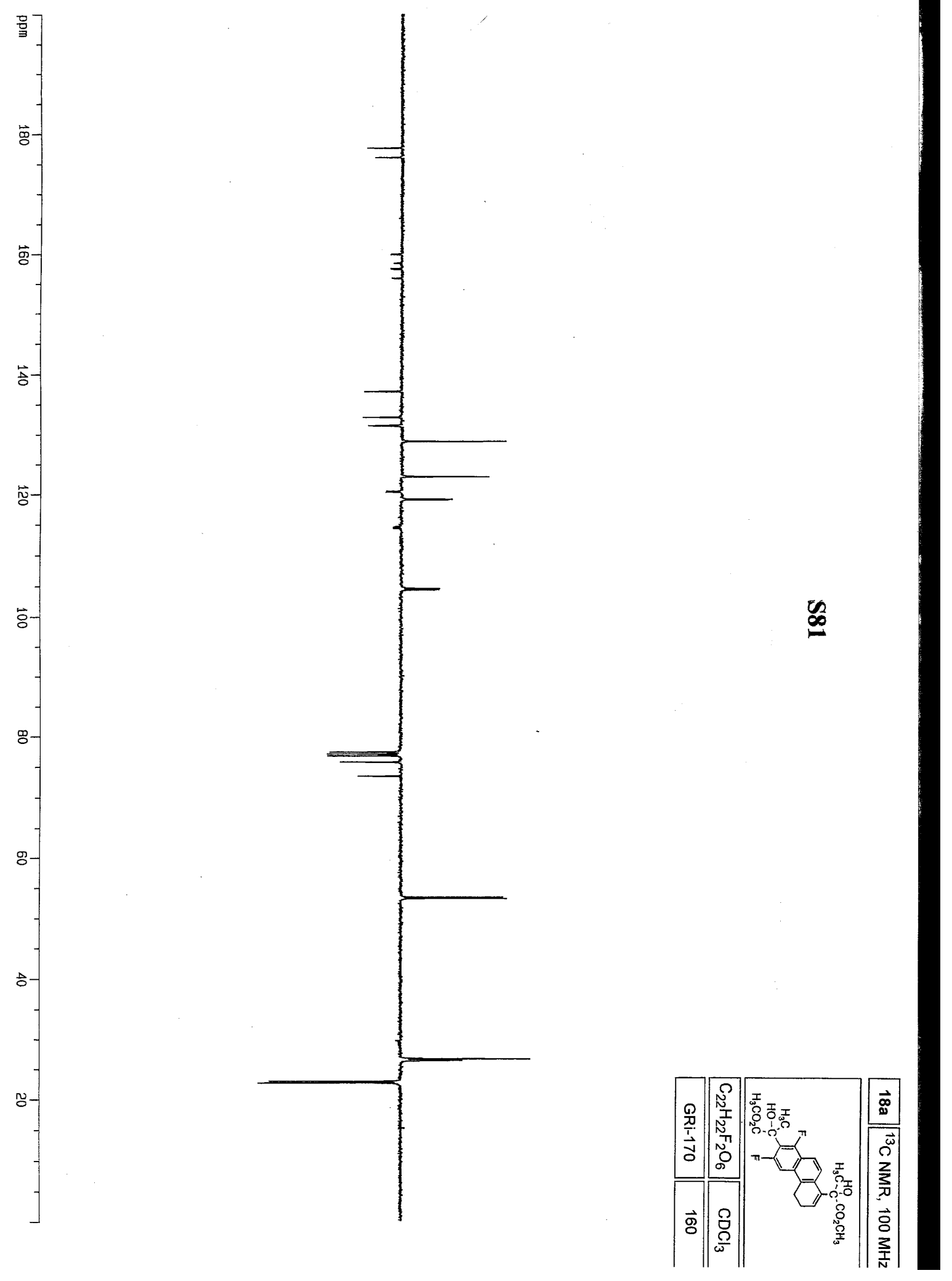

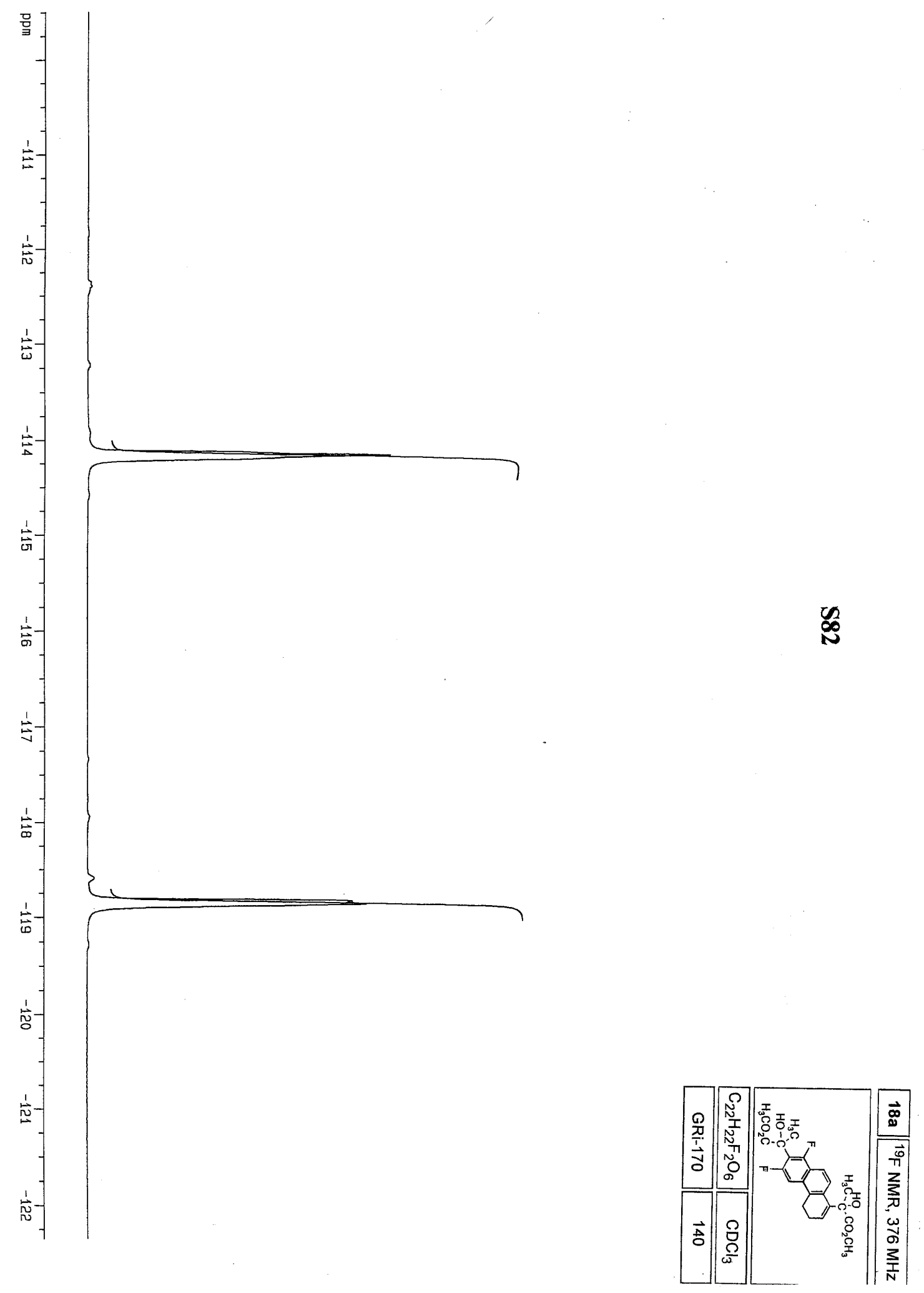

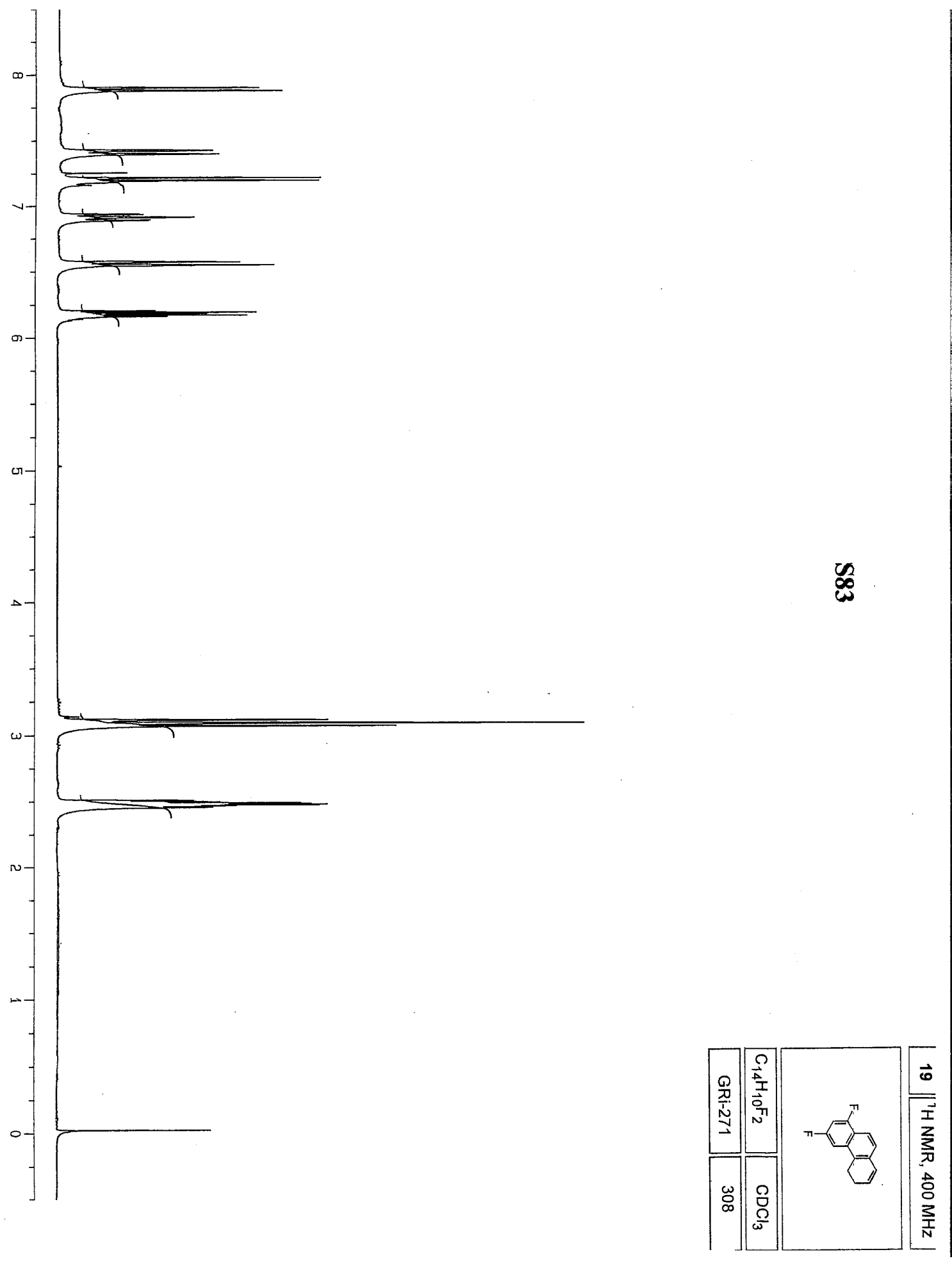

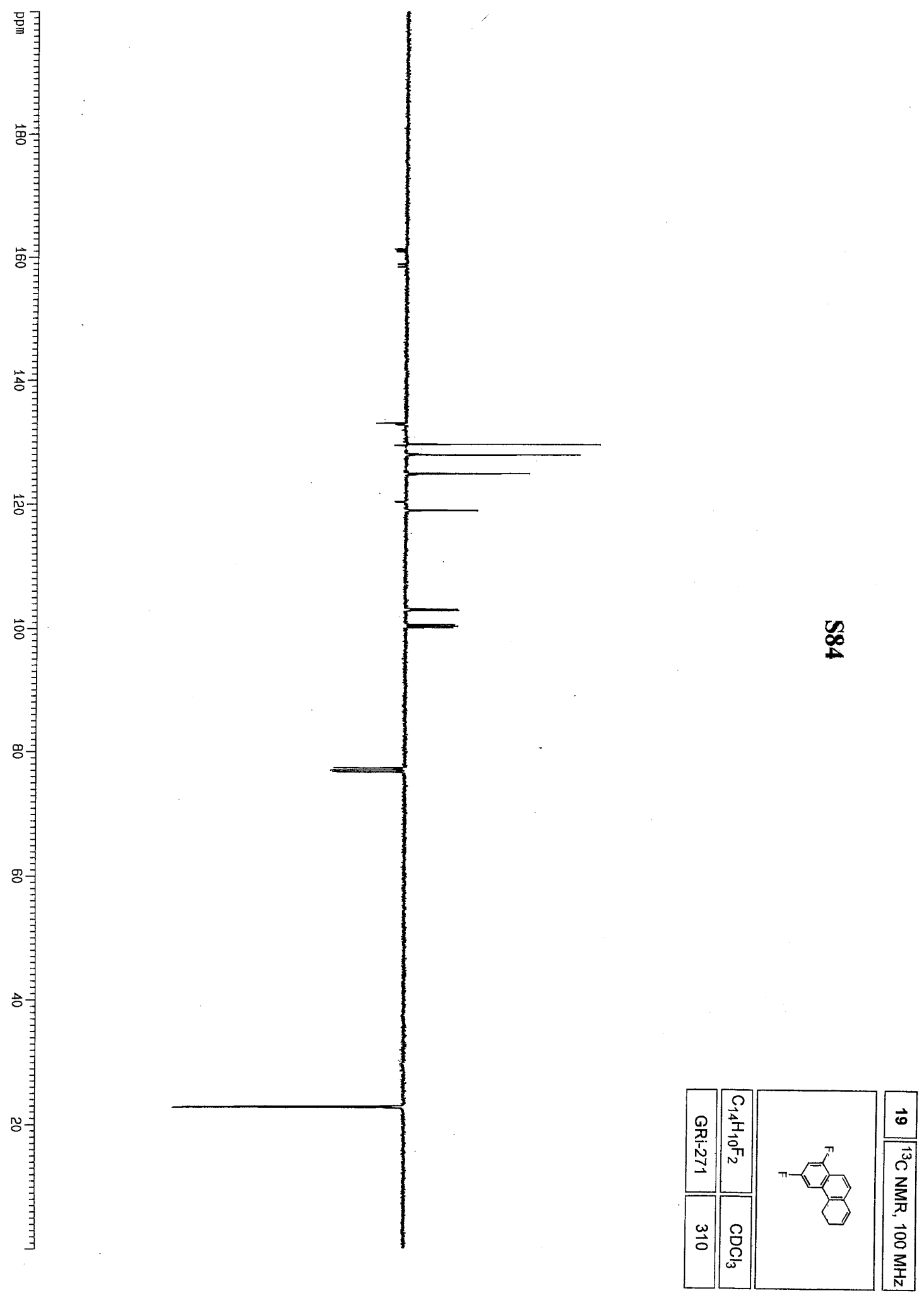


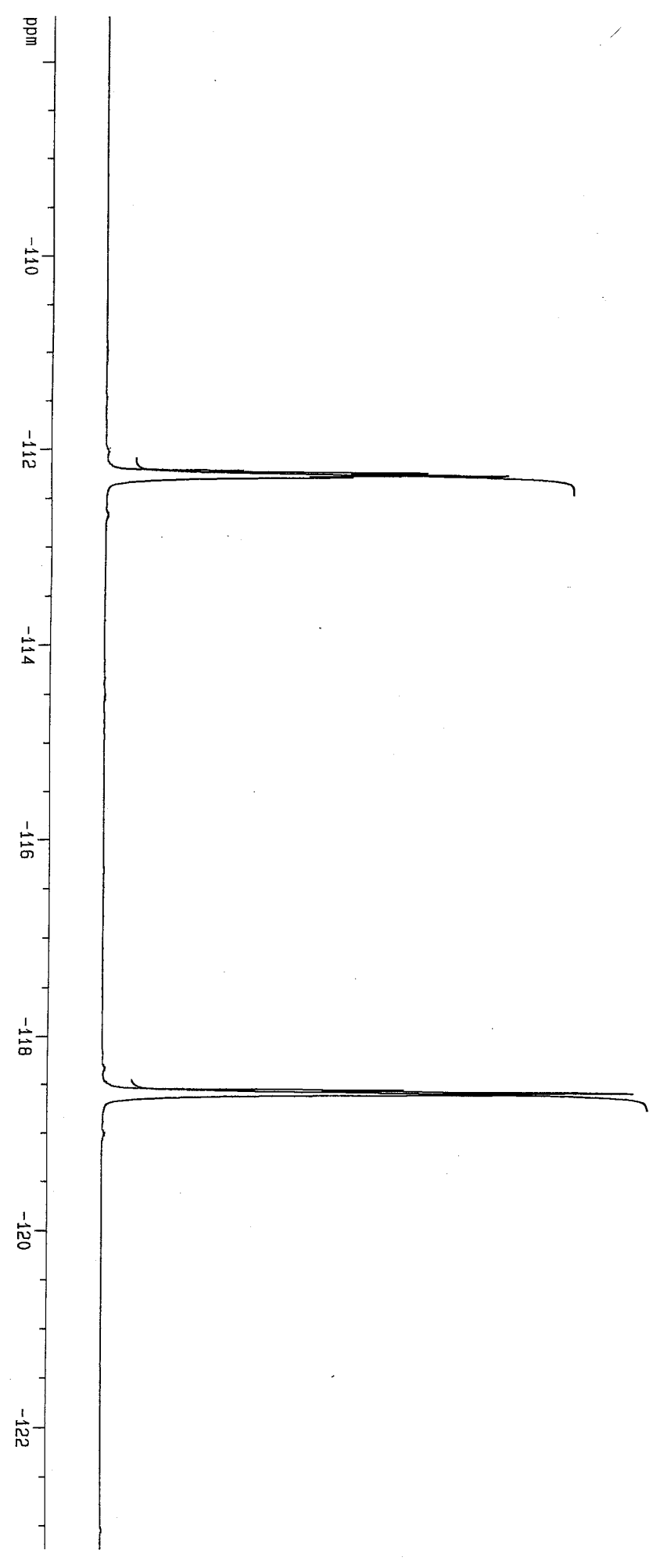

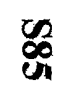

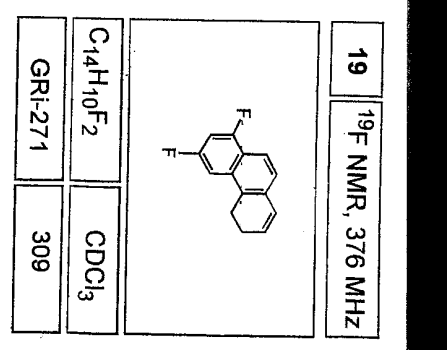




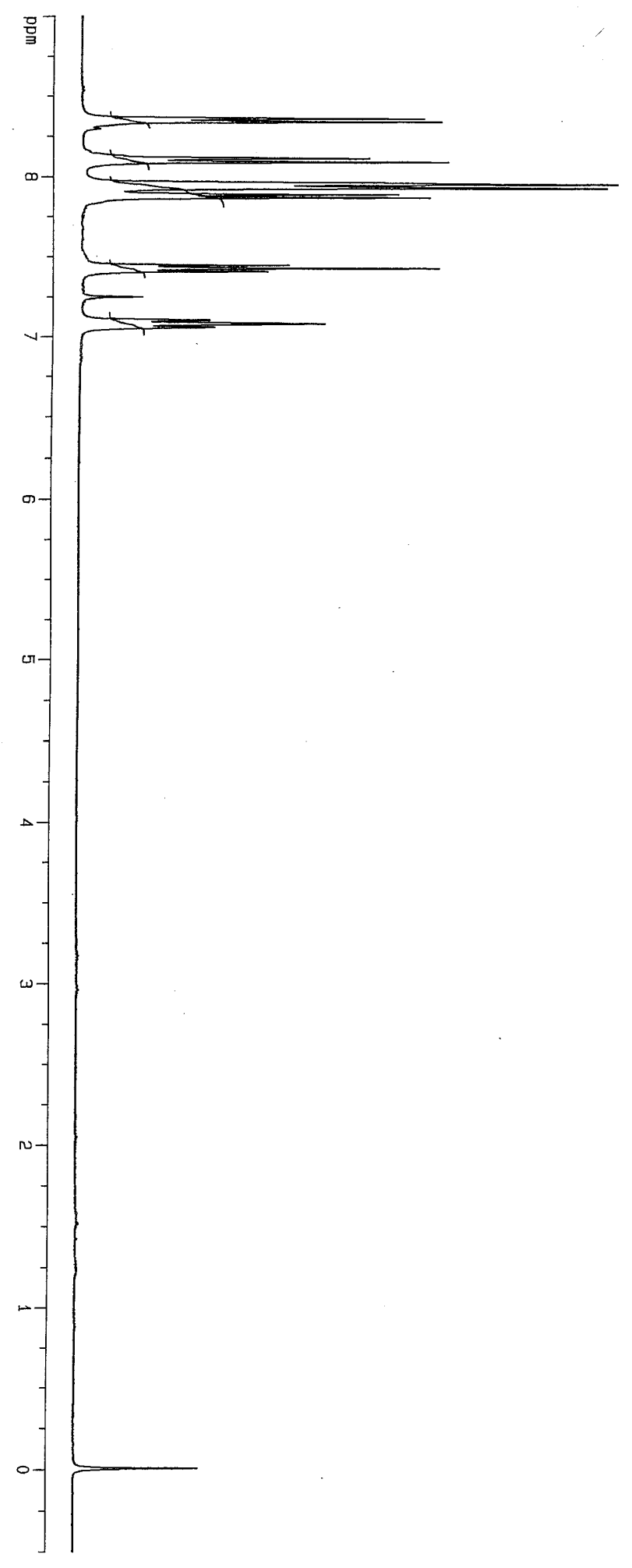

\&

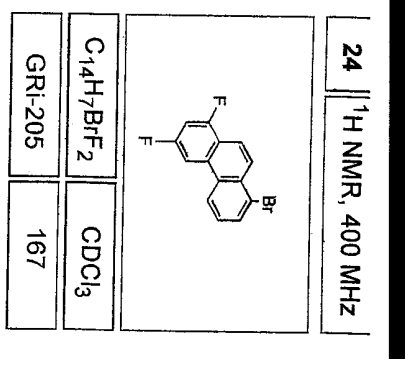




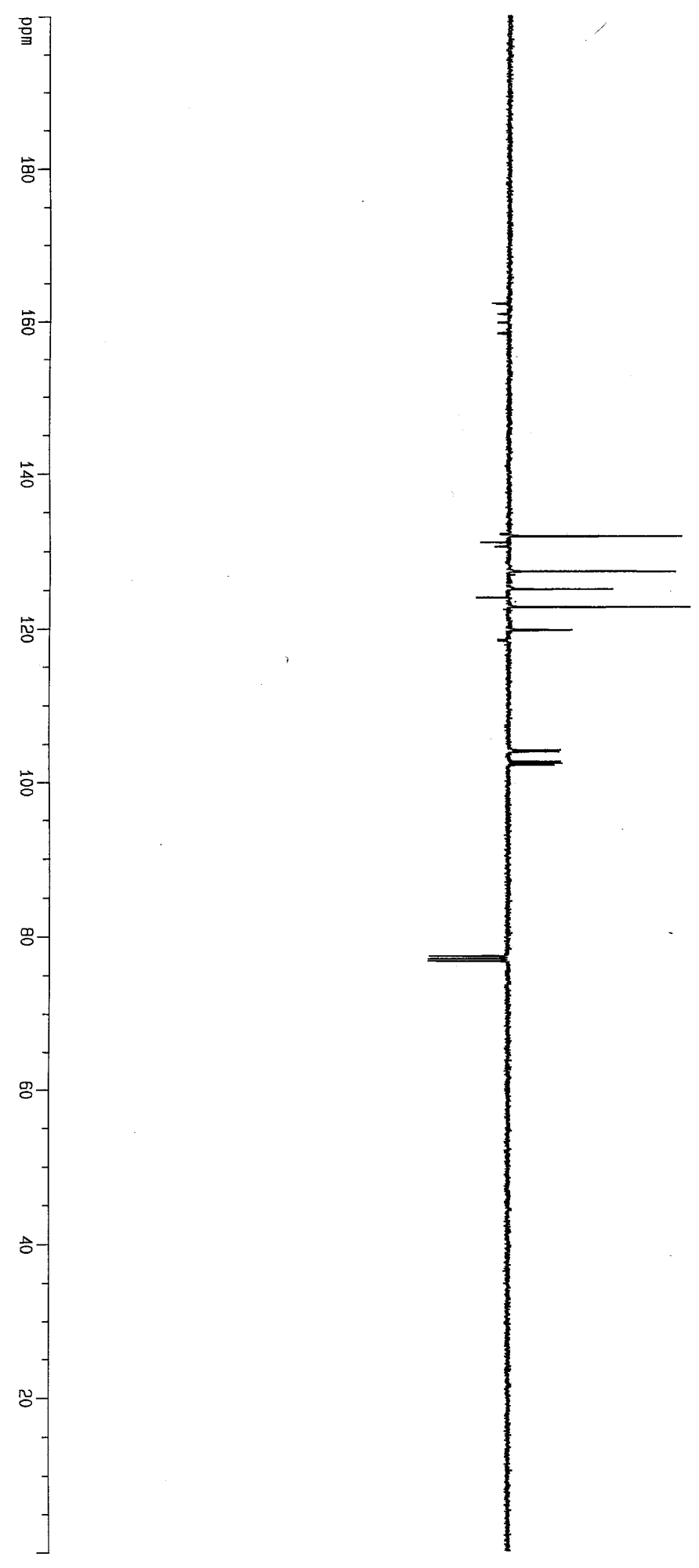

禺

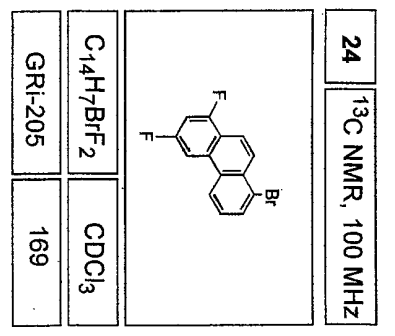




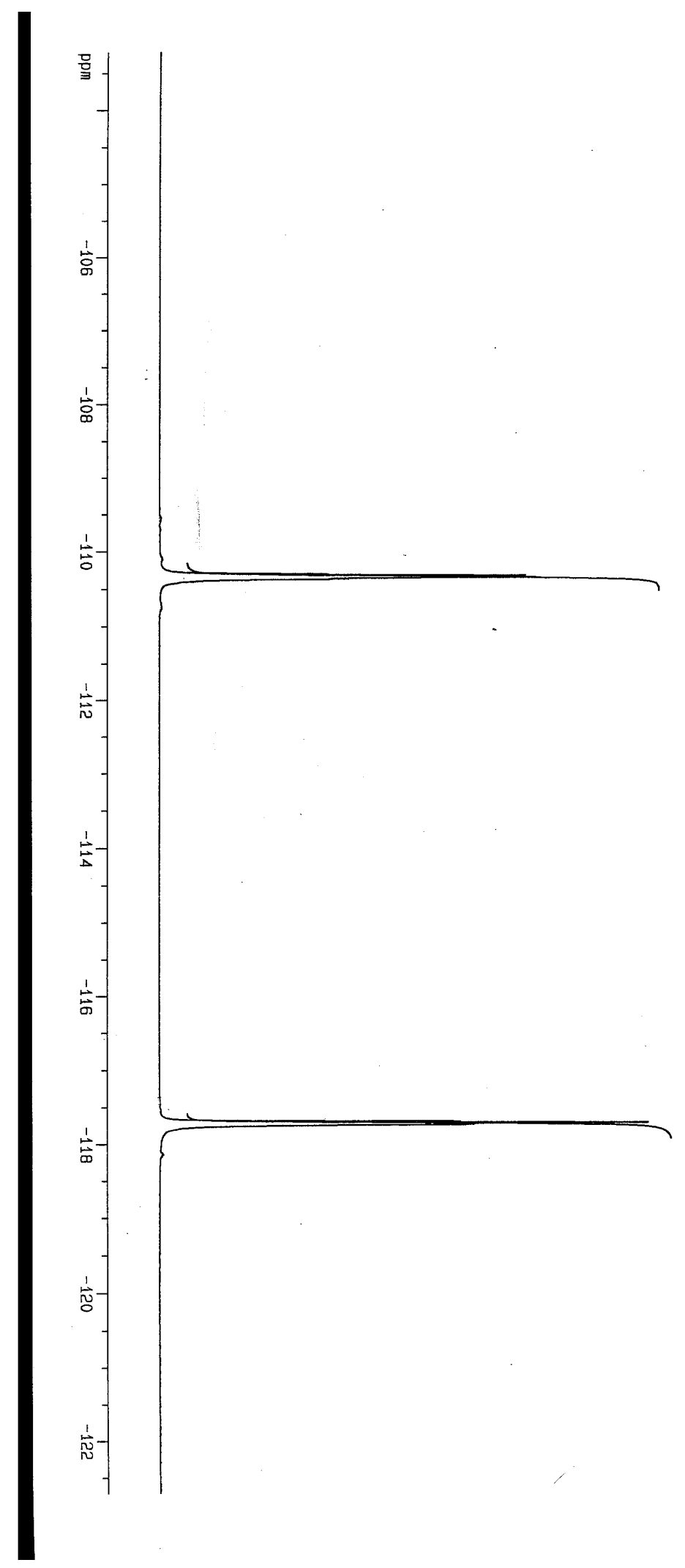

染

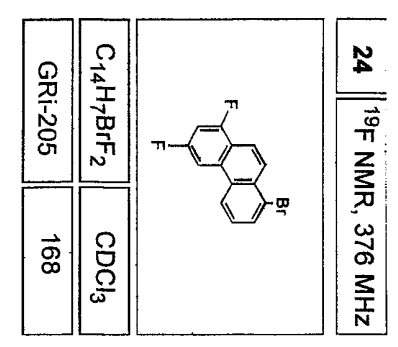



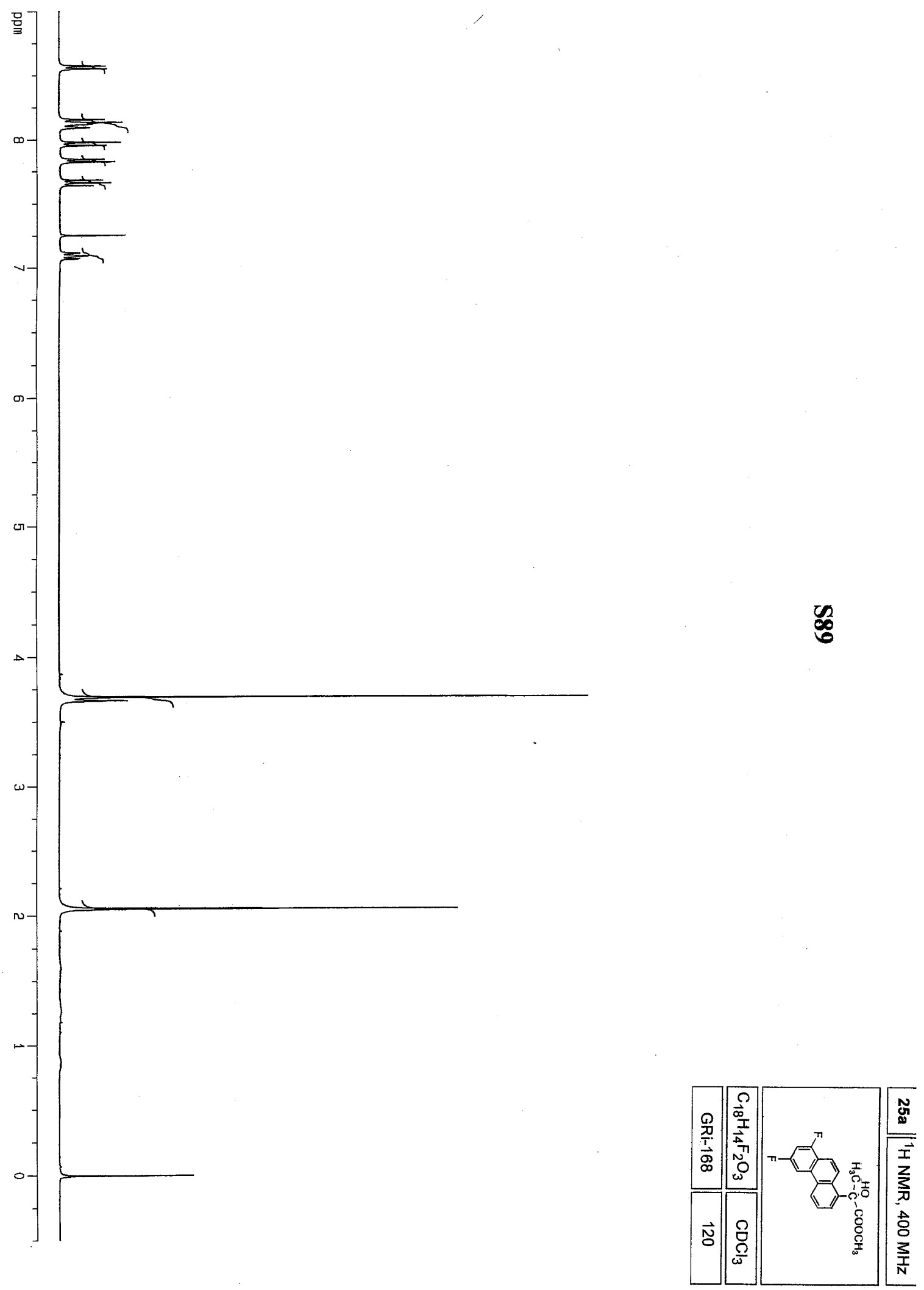


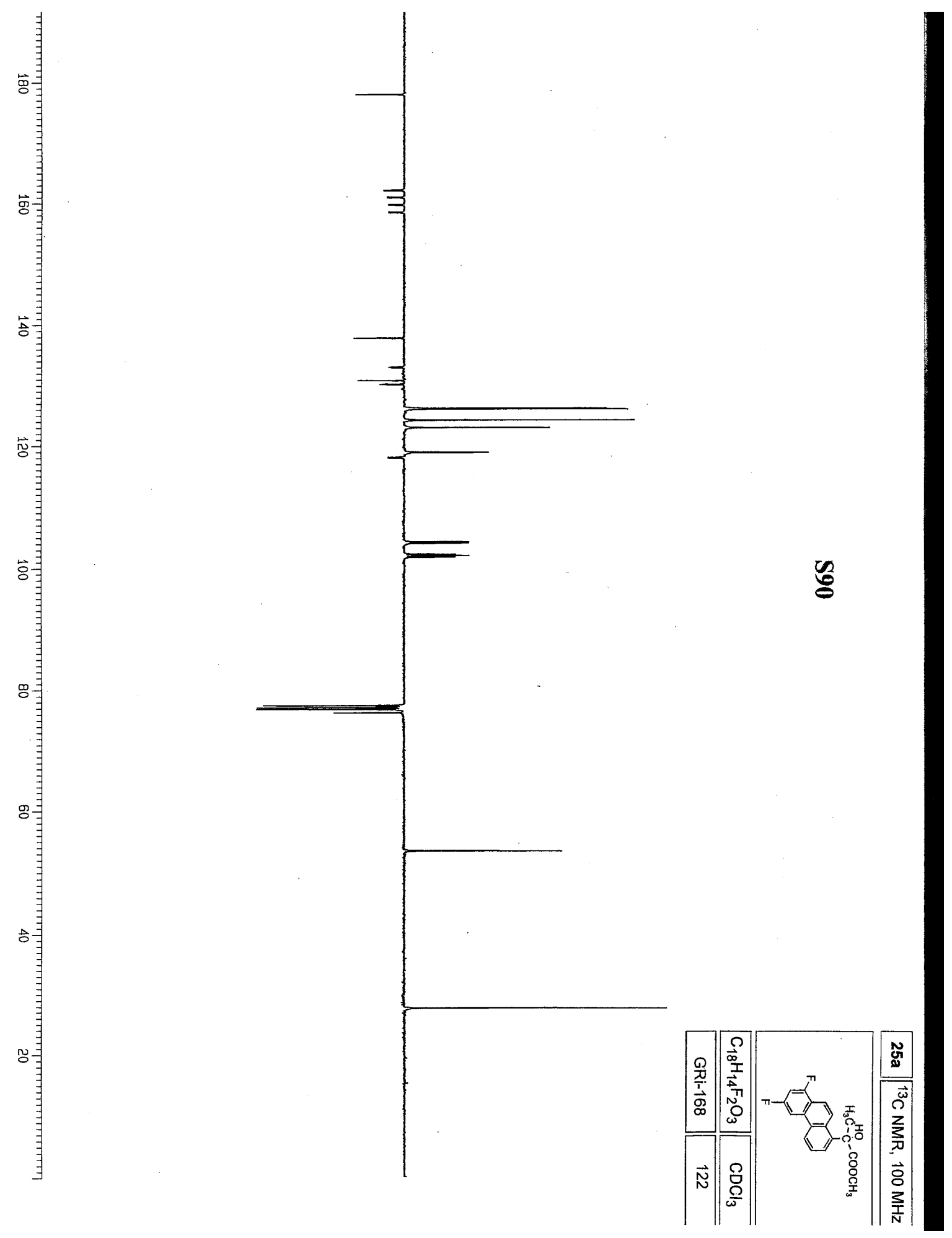




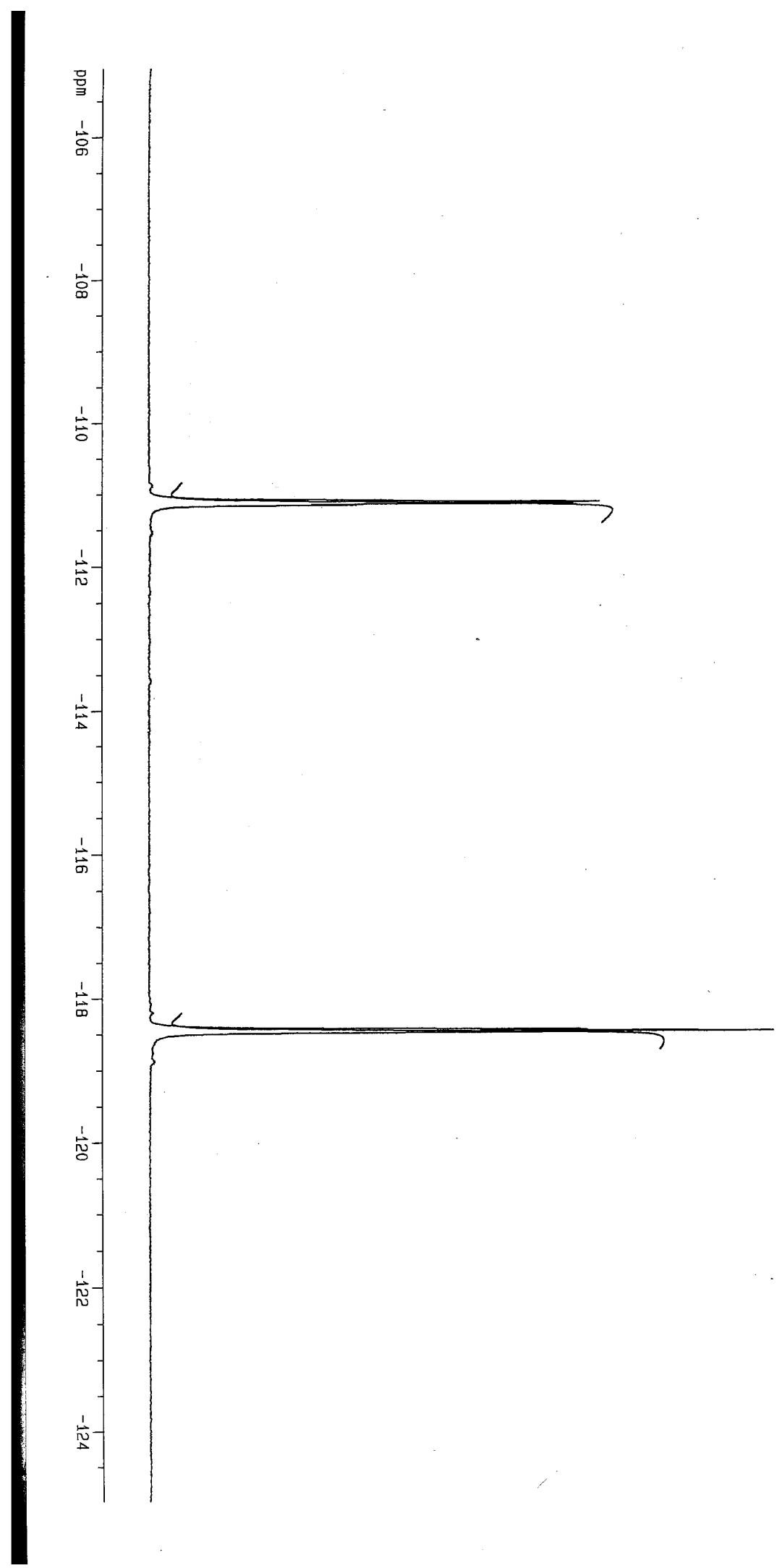

骂

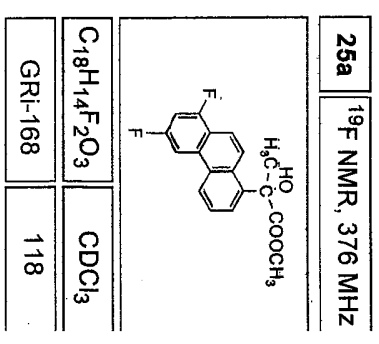



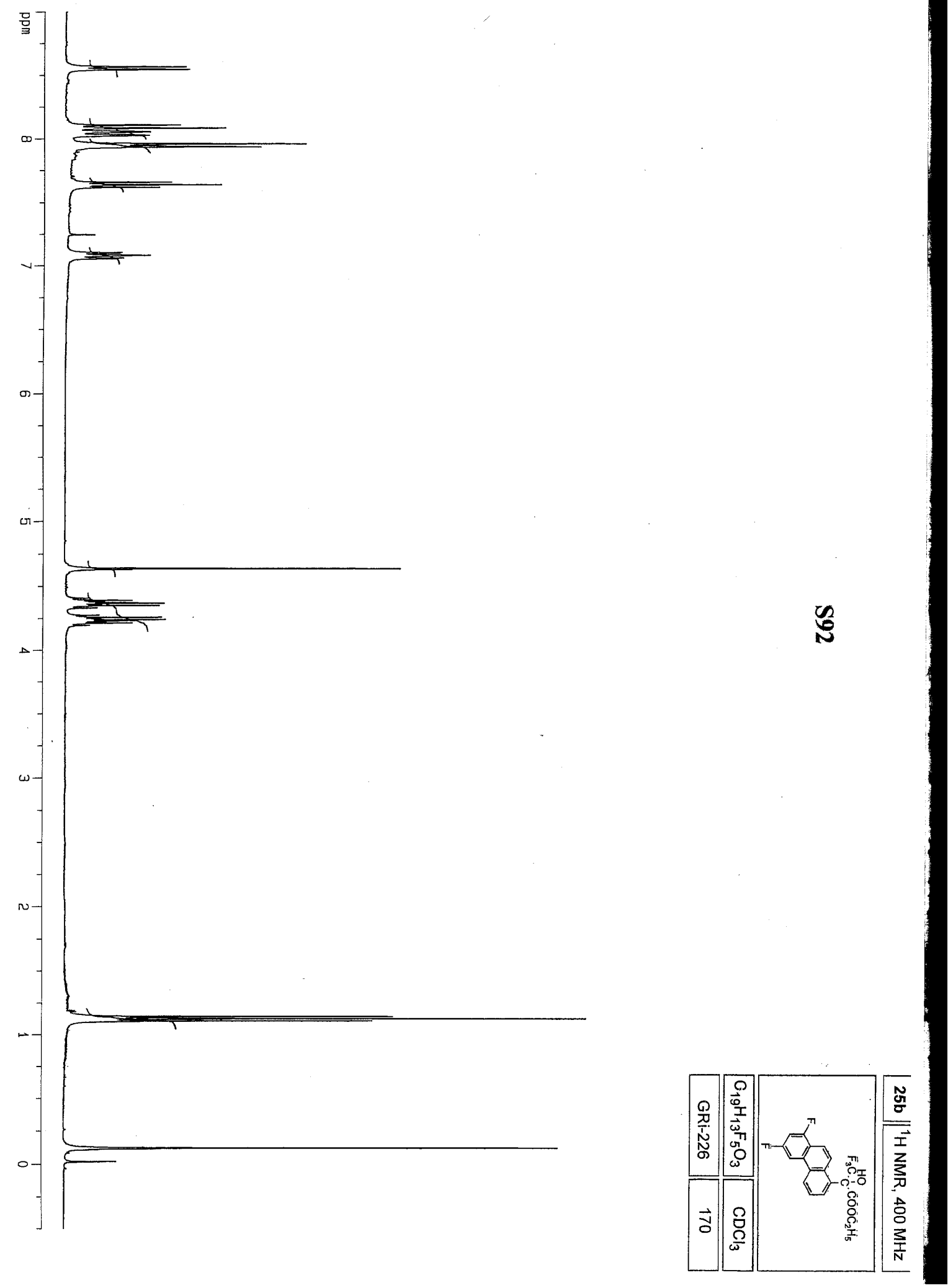

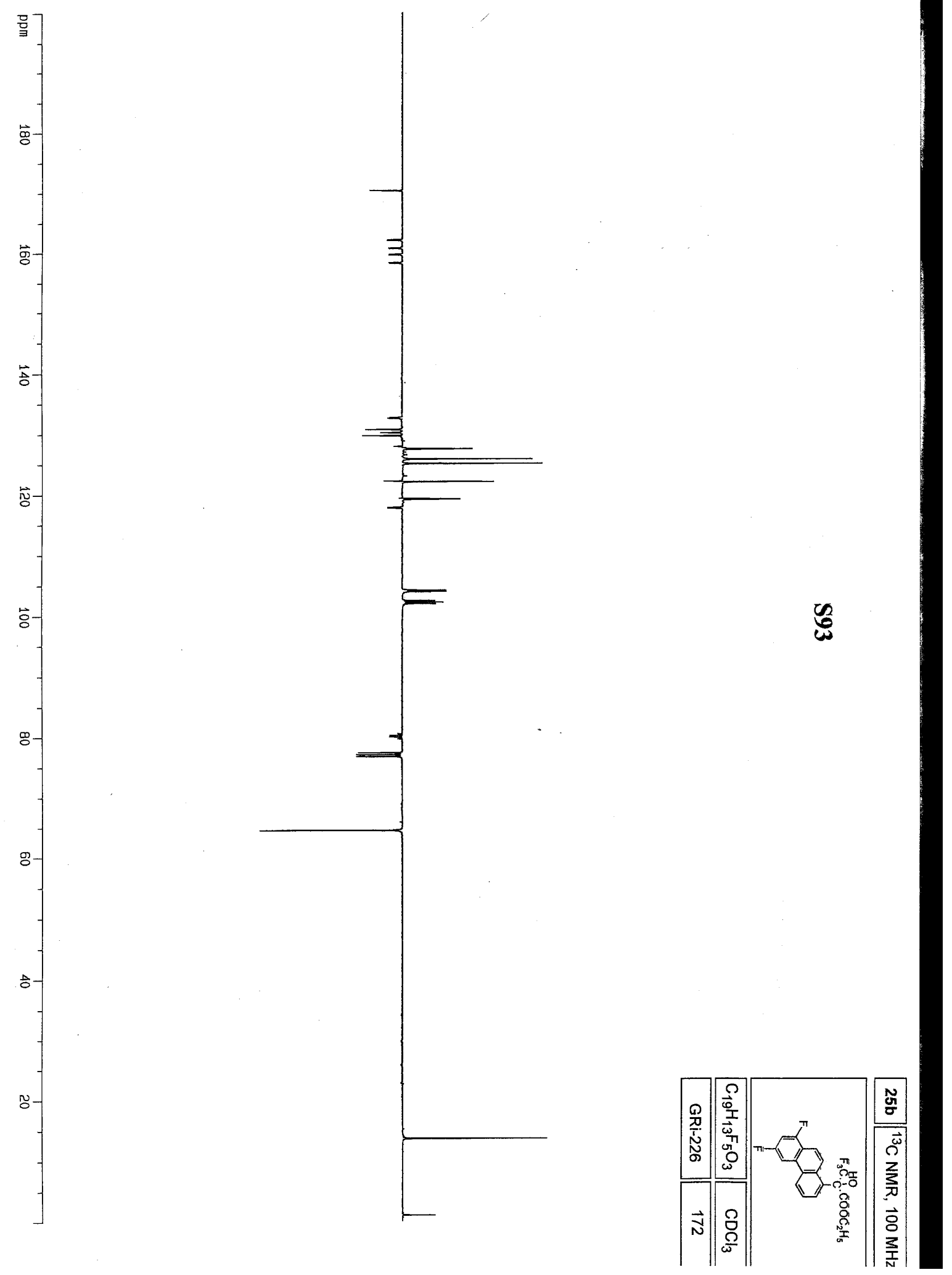


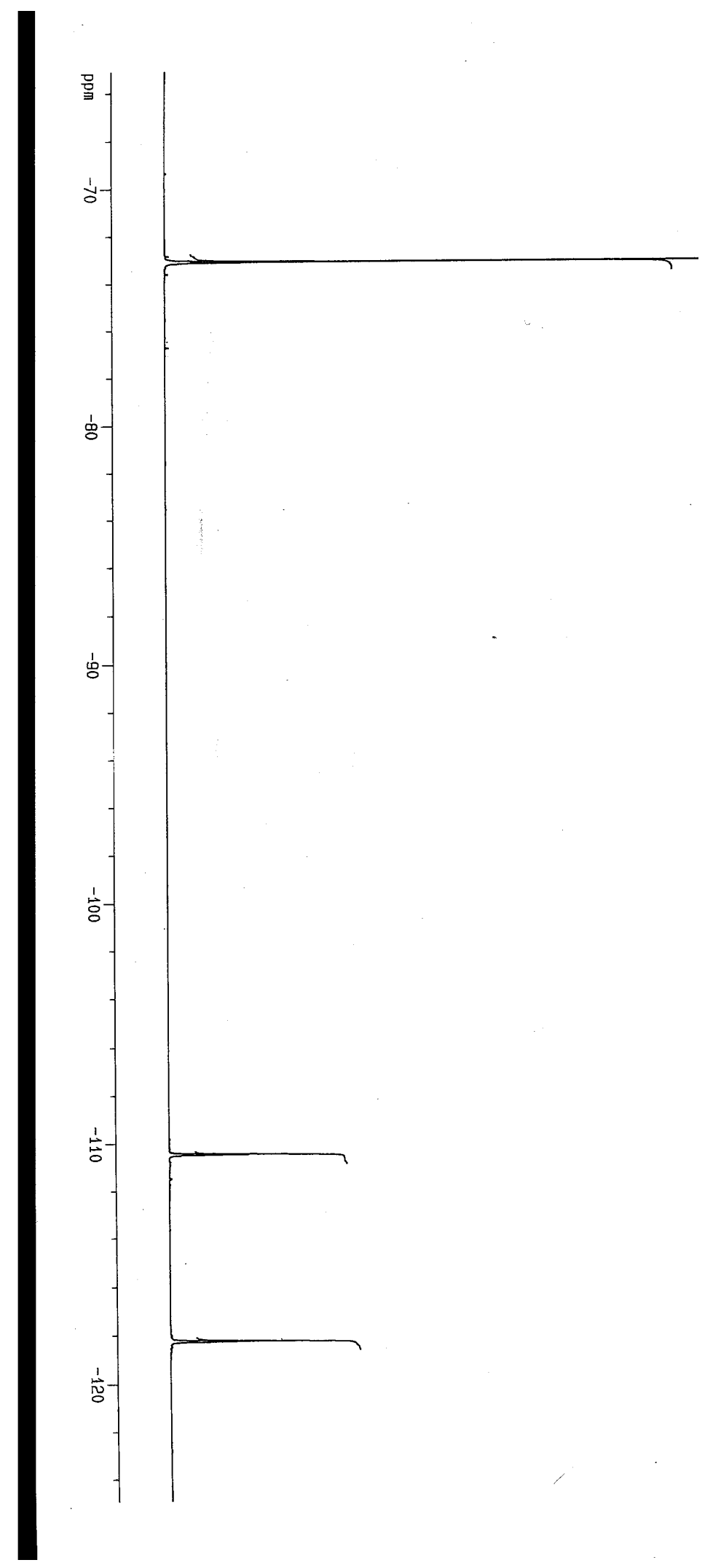

용

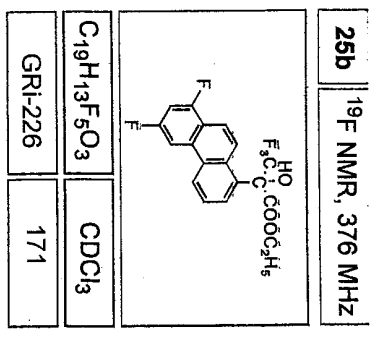




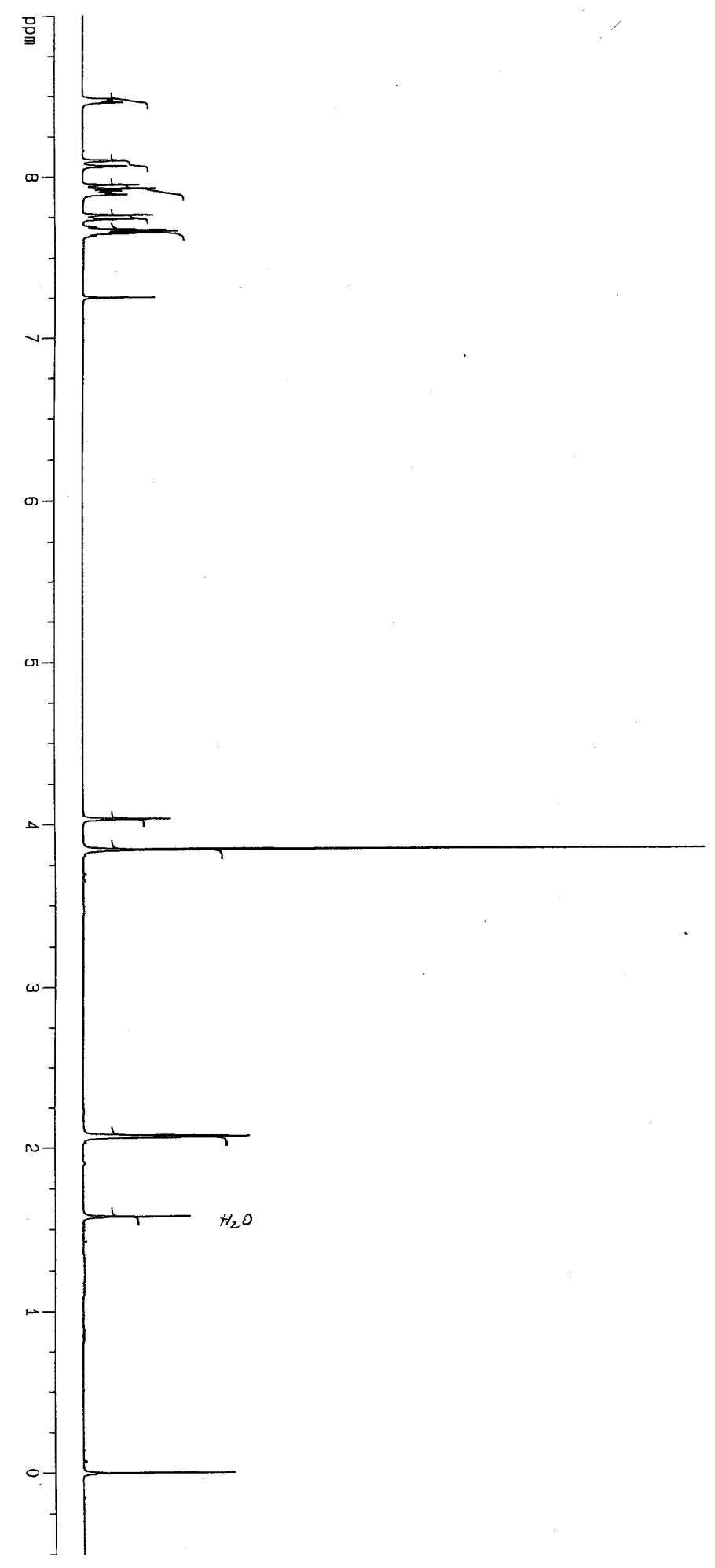

语

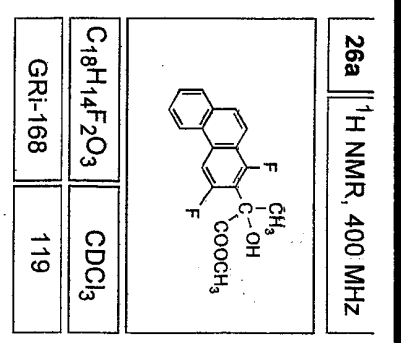



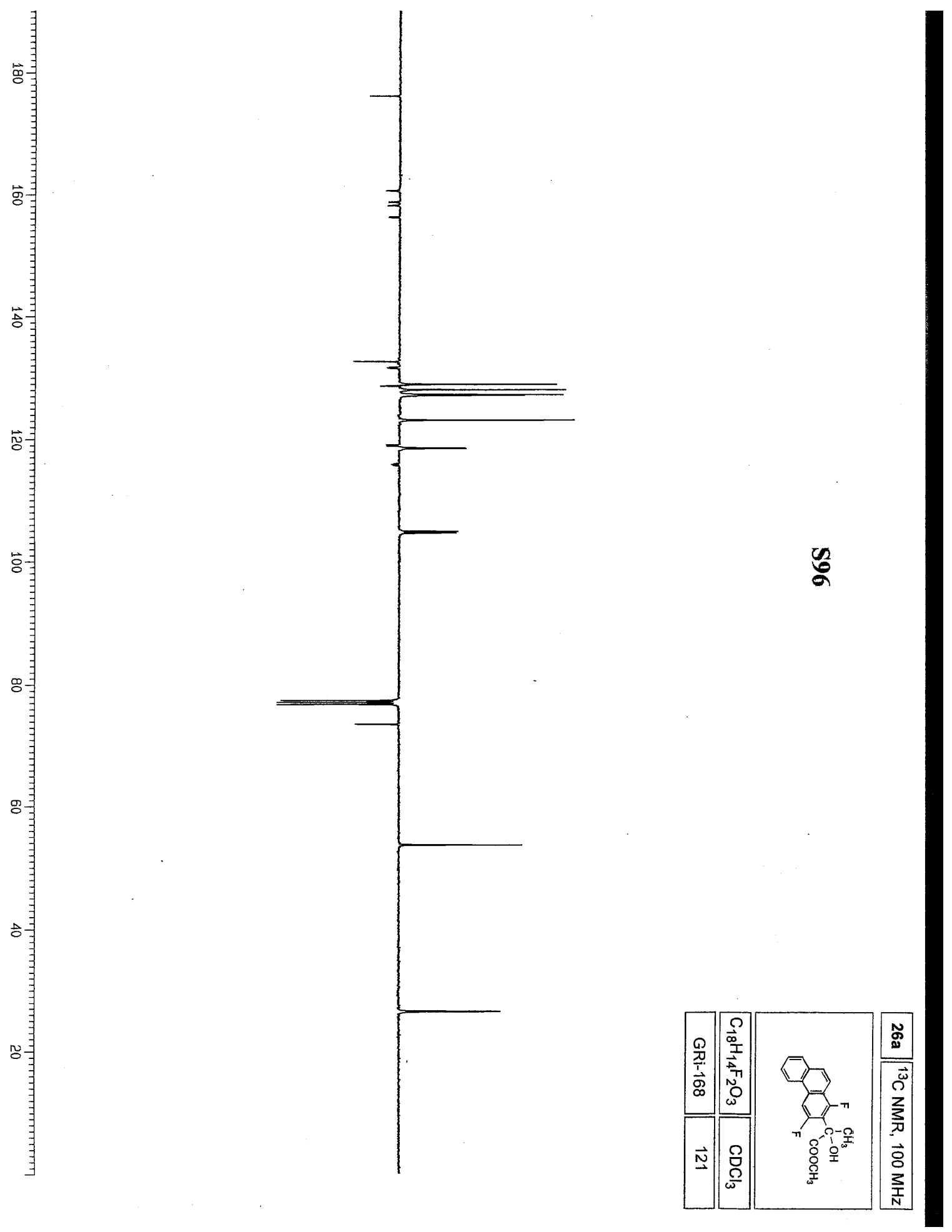


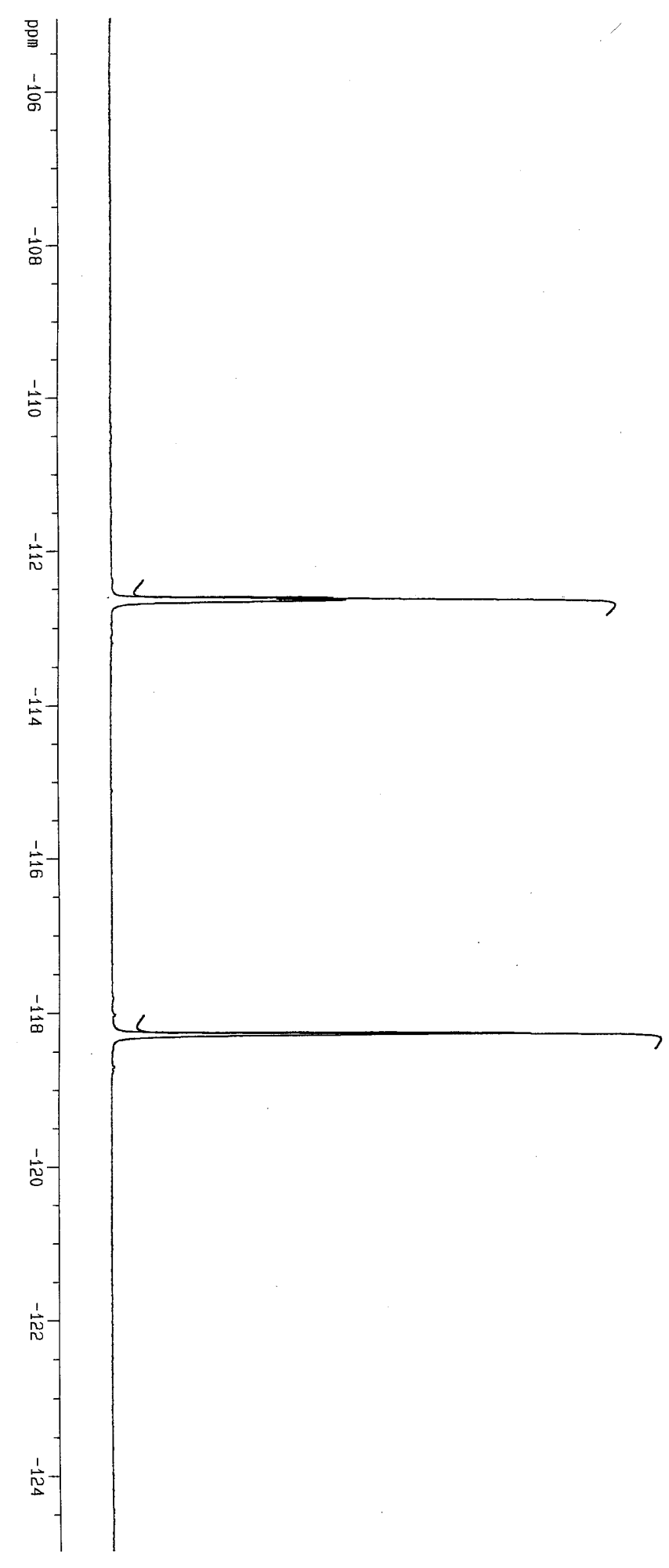

50

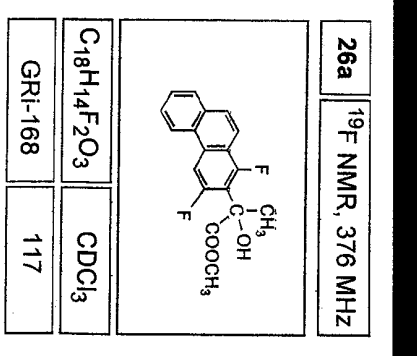




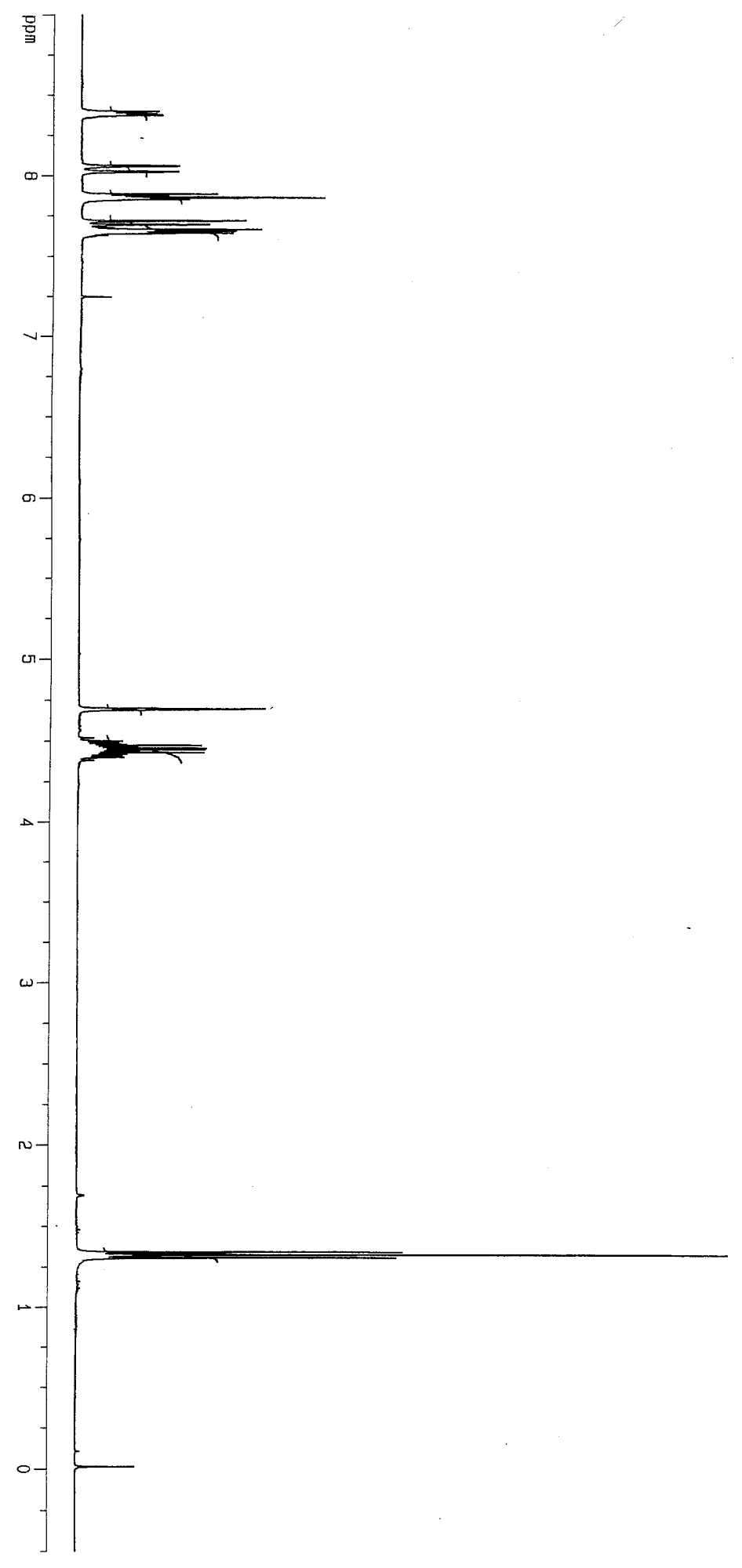




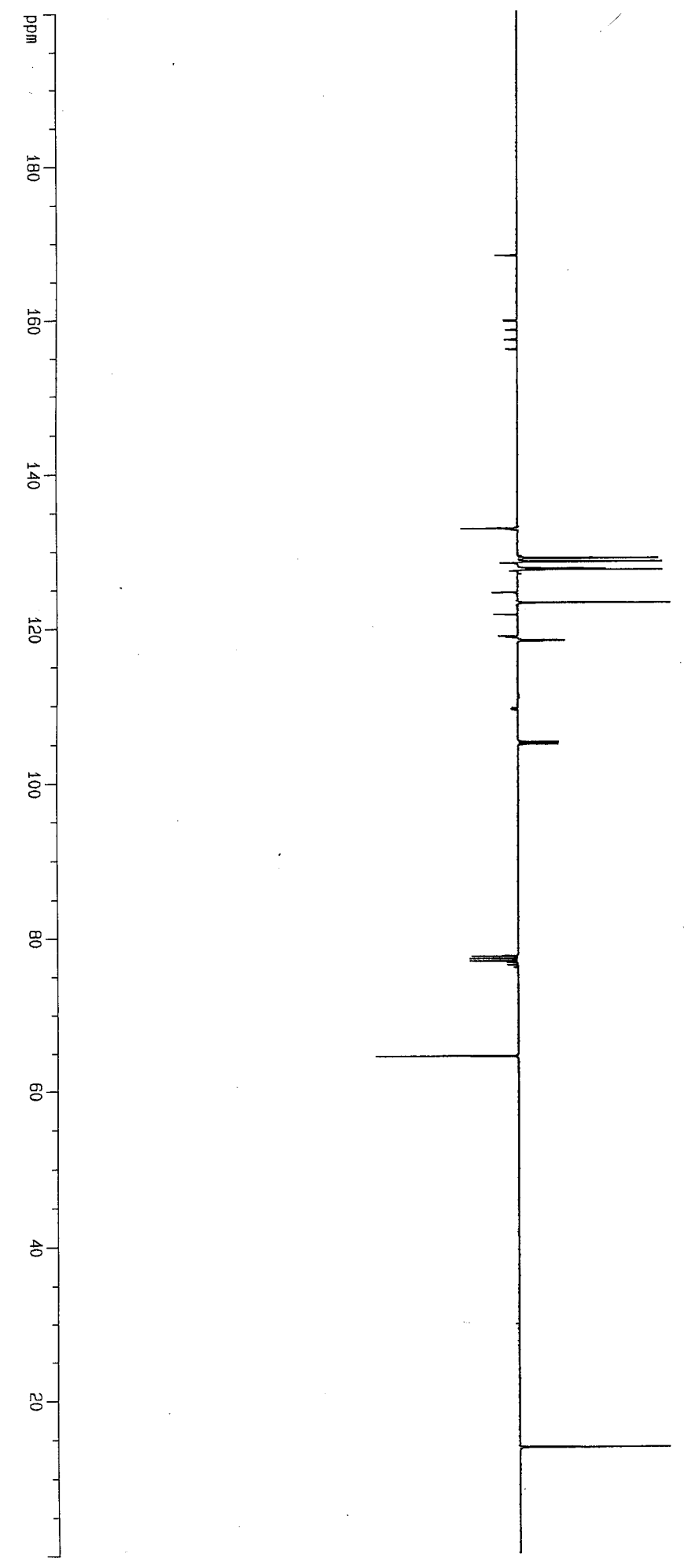

క气

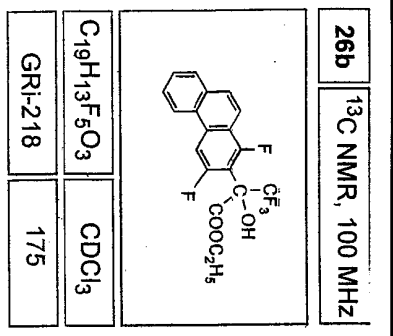



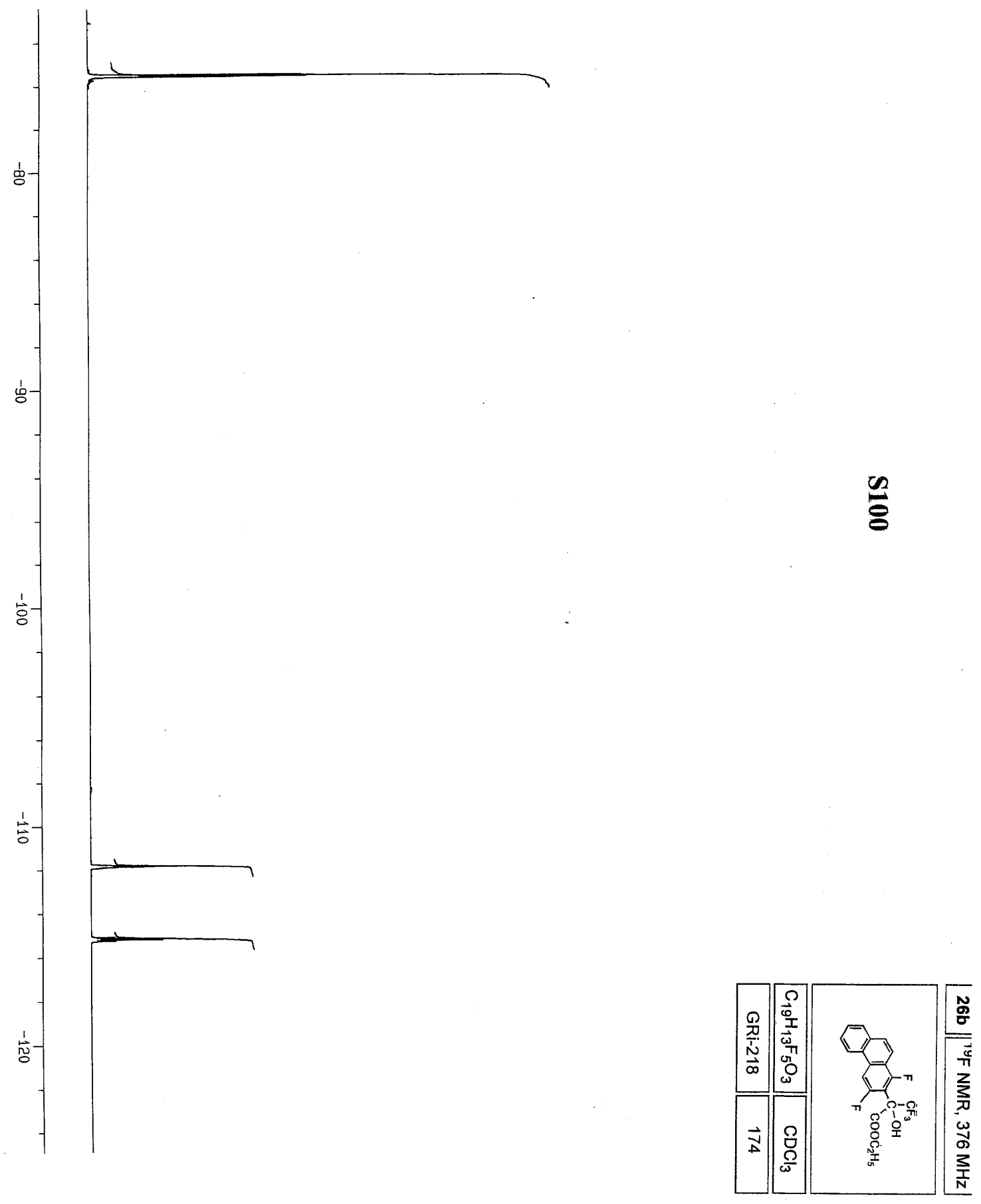


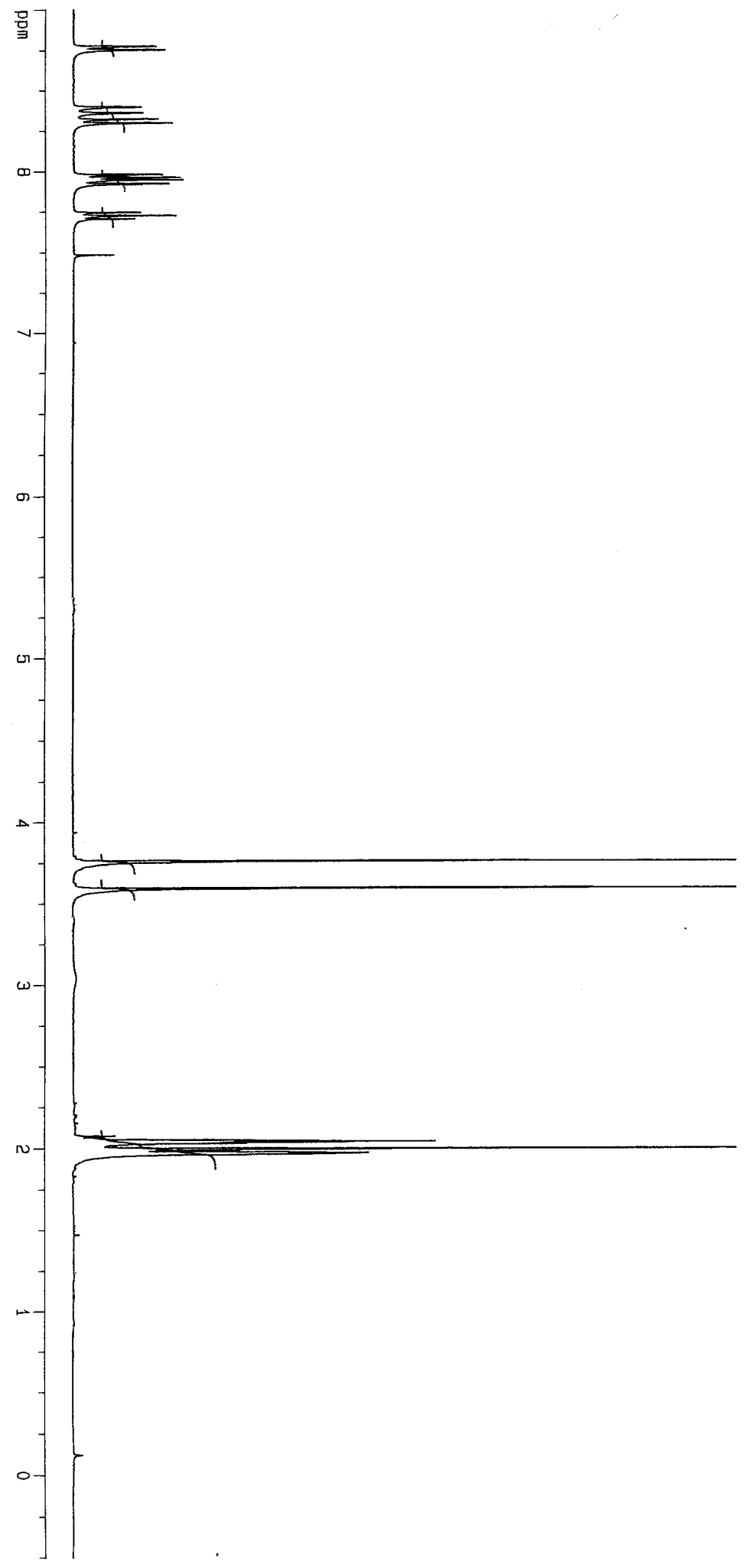

0

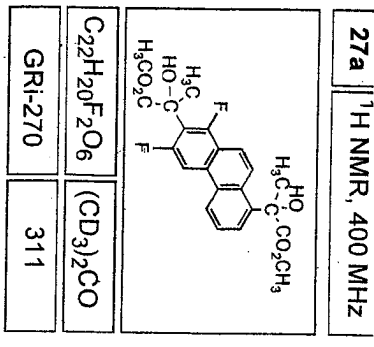




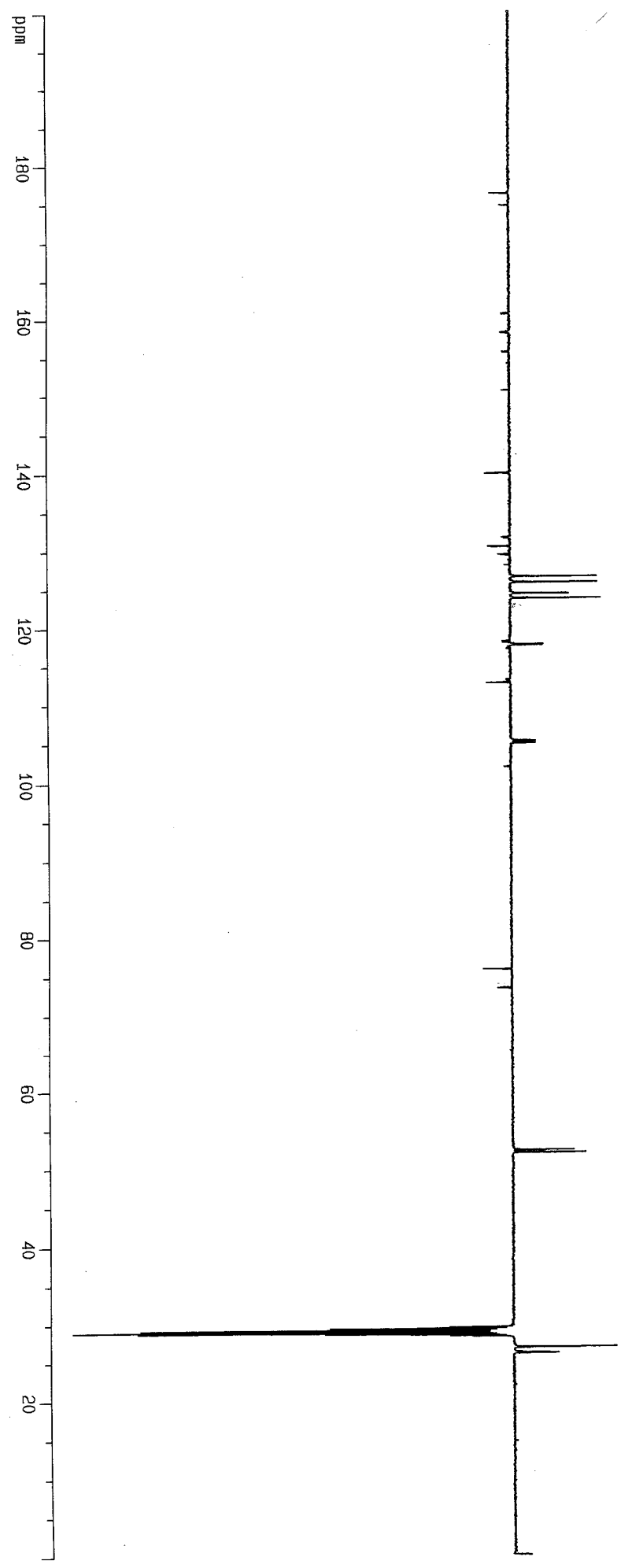

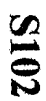

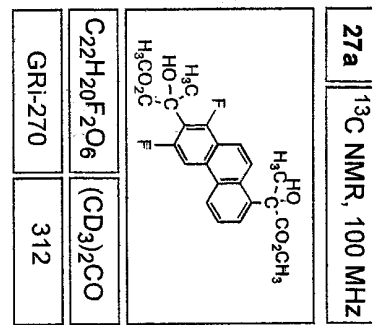




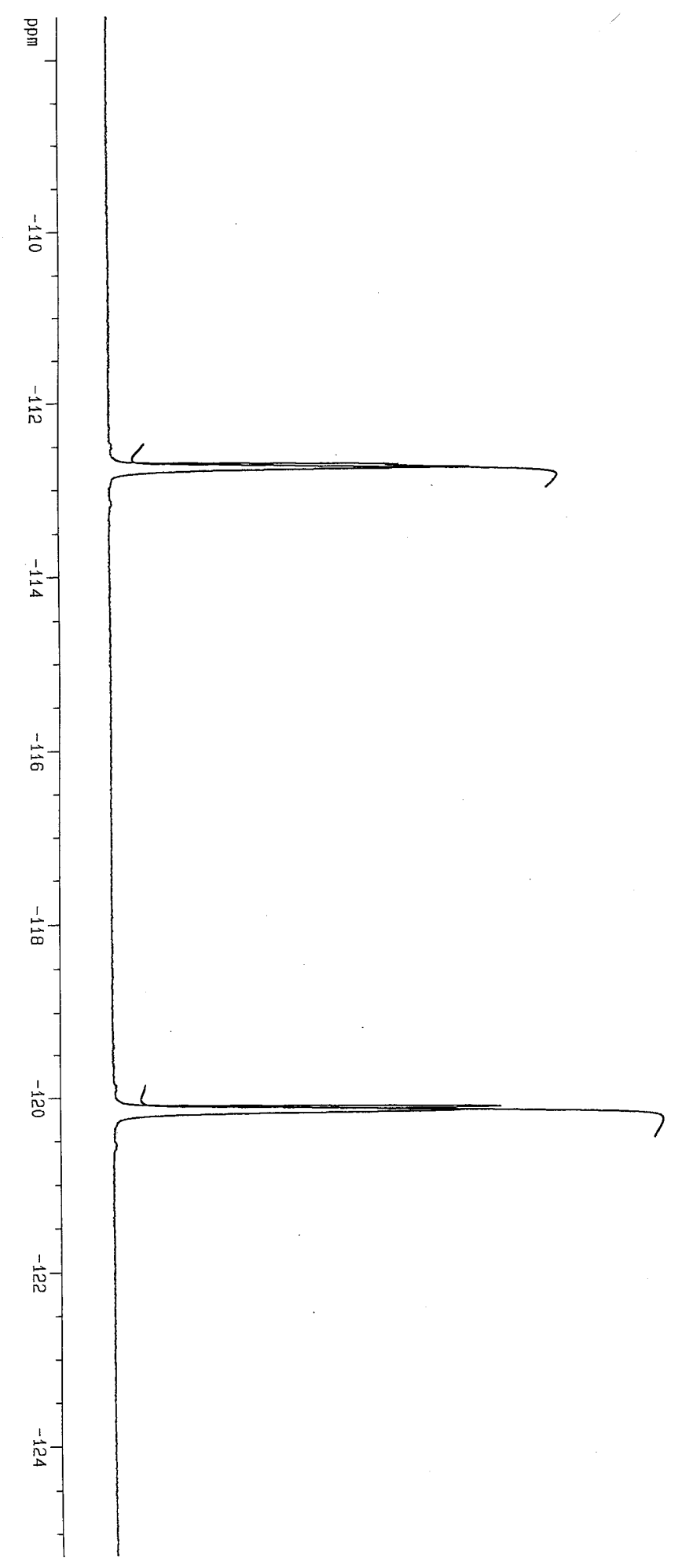

$\infty$

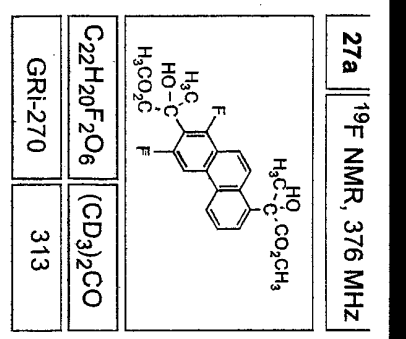




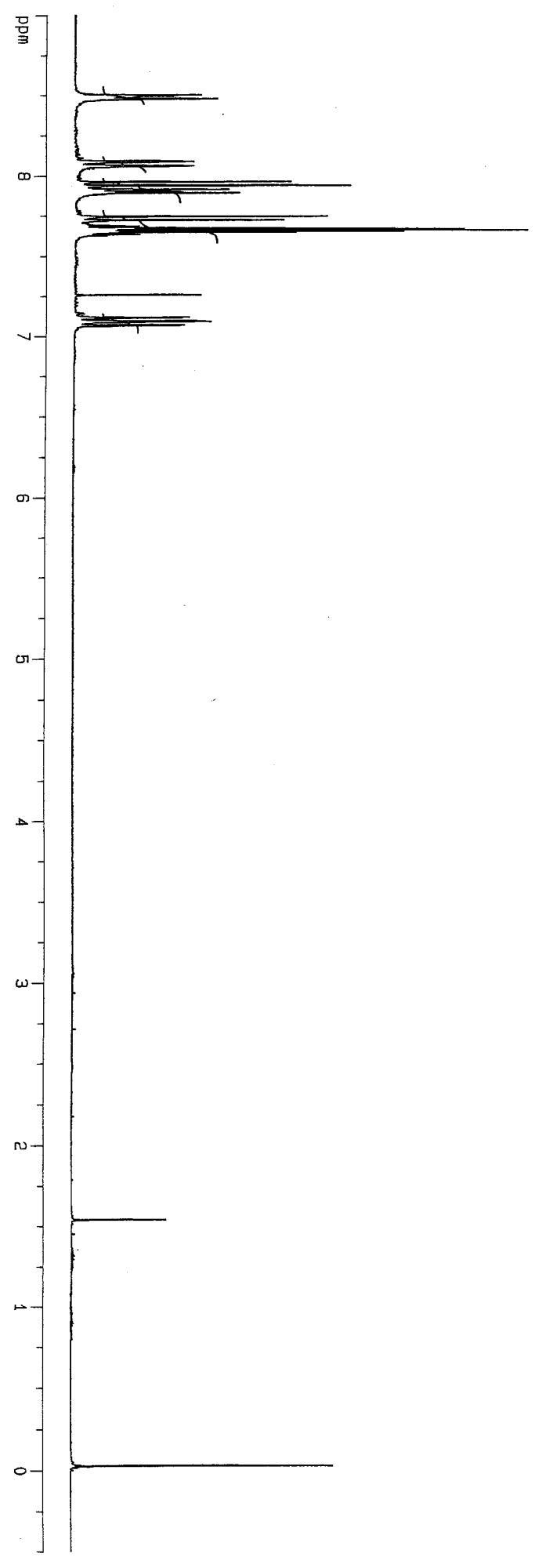

$\frac{0}{8}$
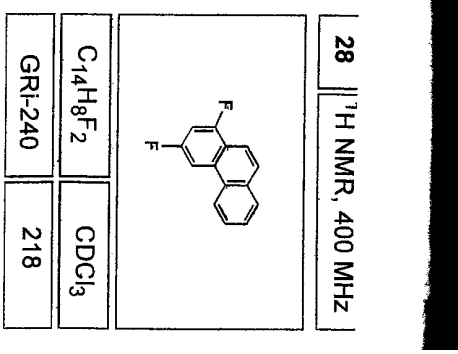


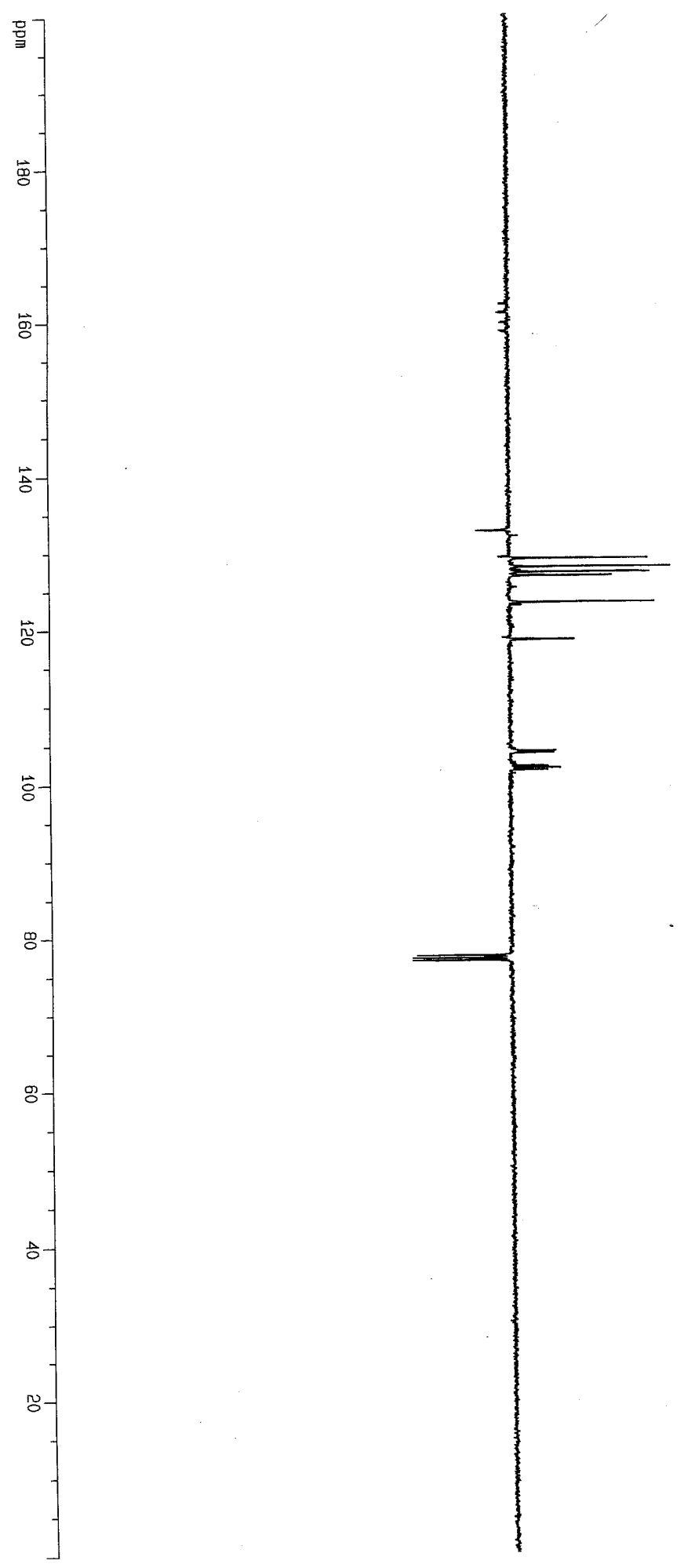

$e^{0}$

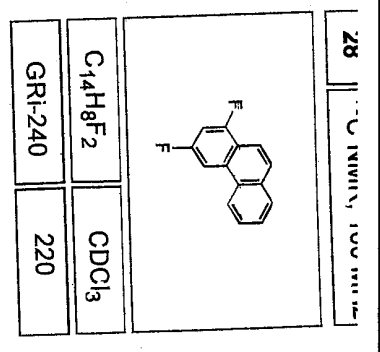




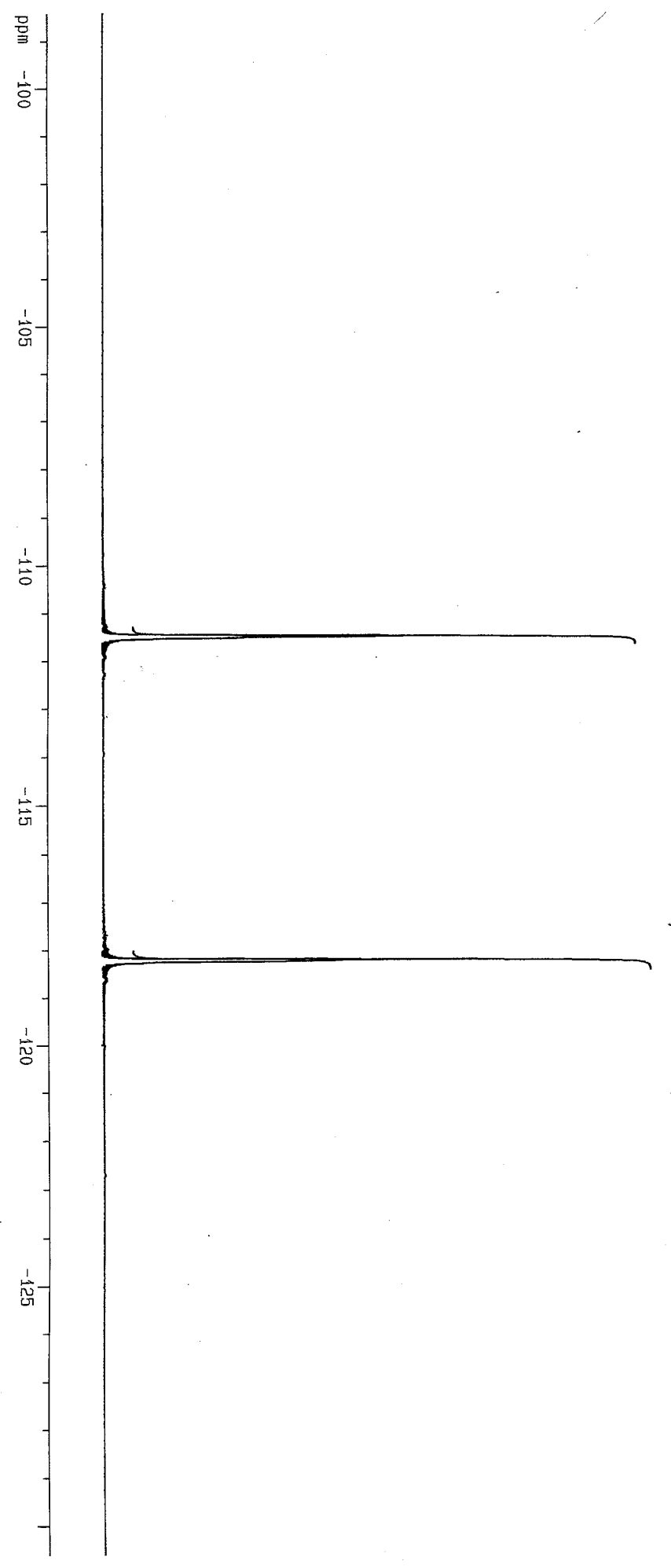

$\frac{2}{8}$

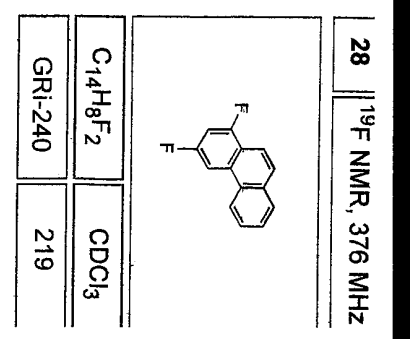




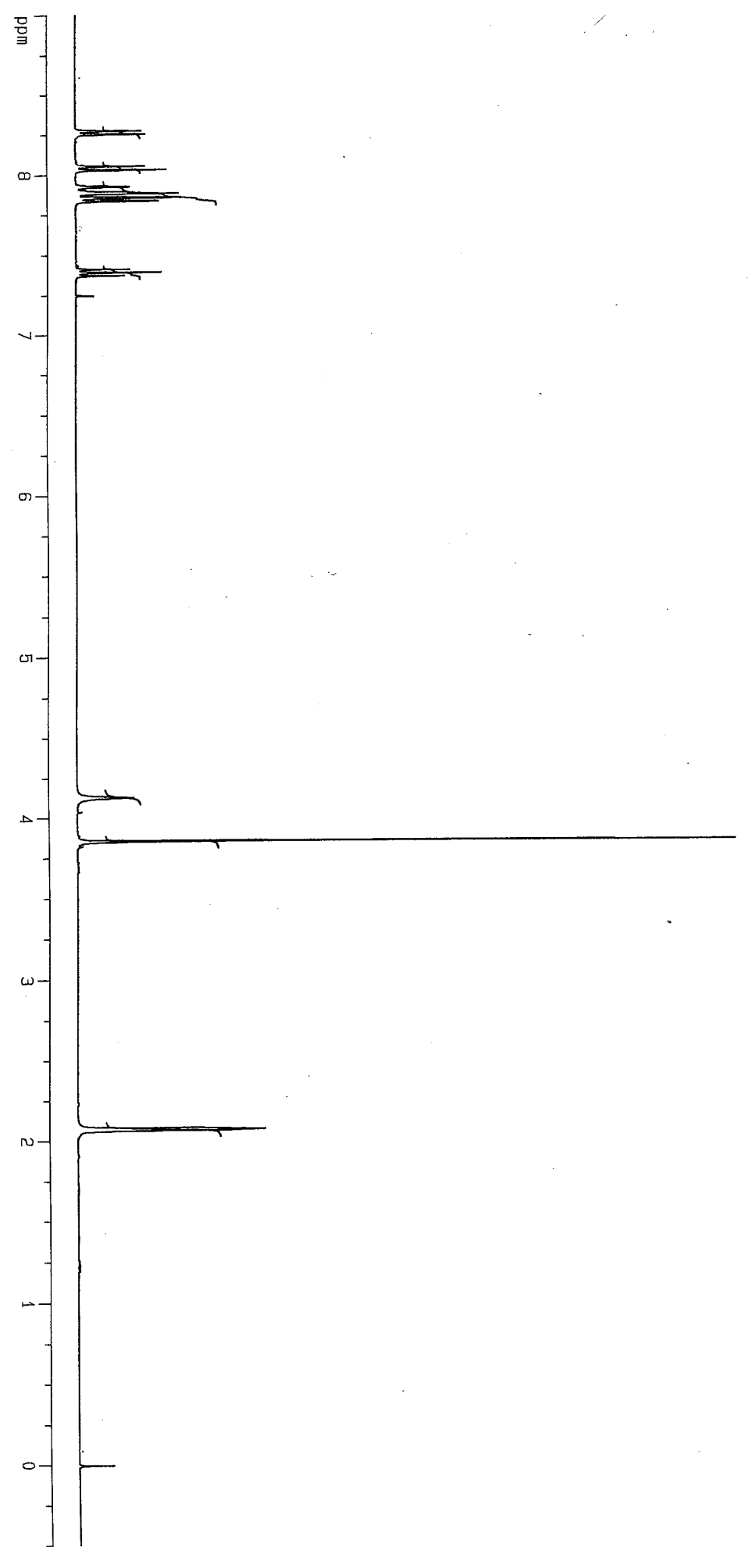

$\frac{6}{3}$

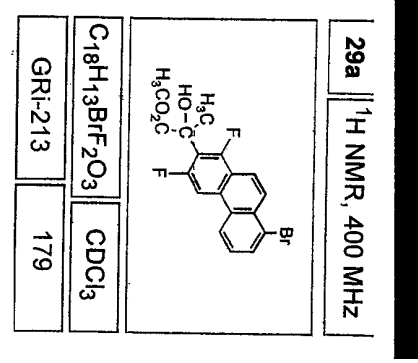



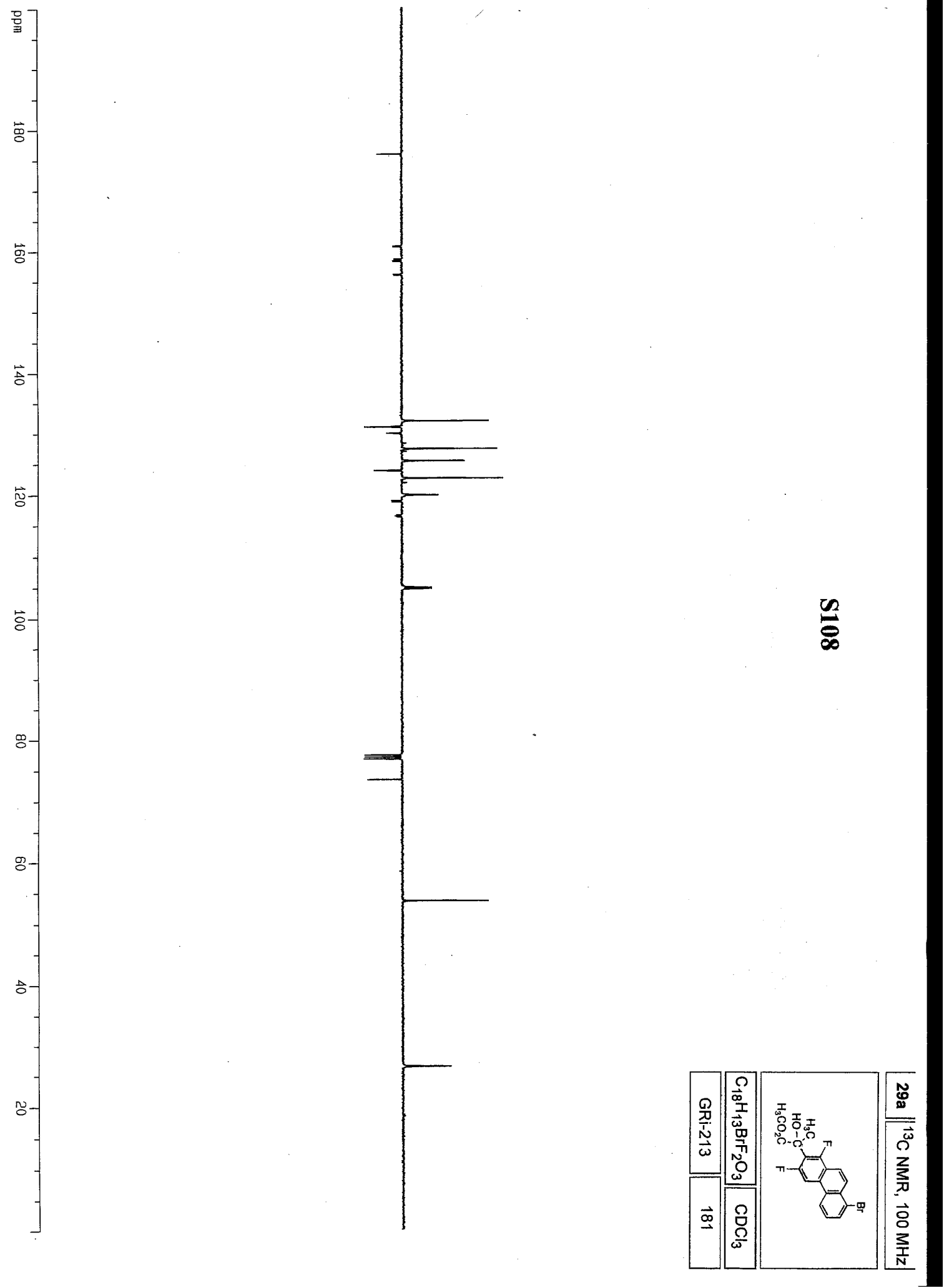

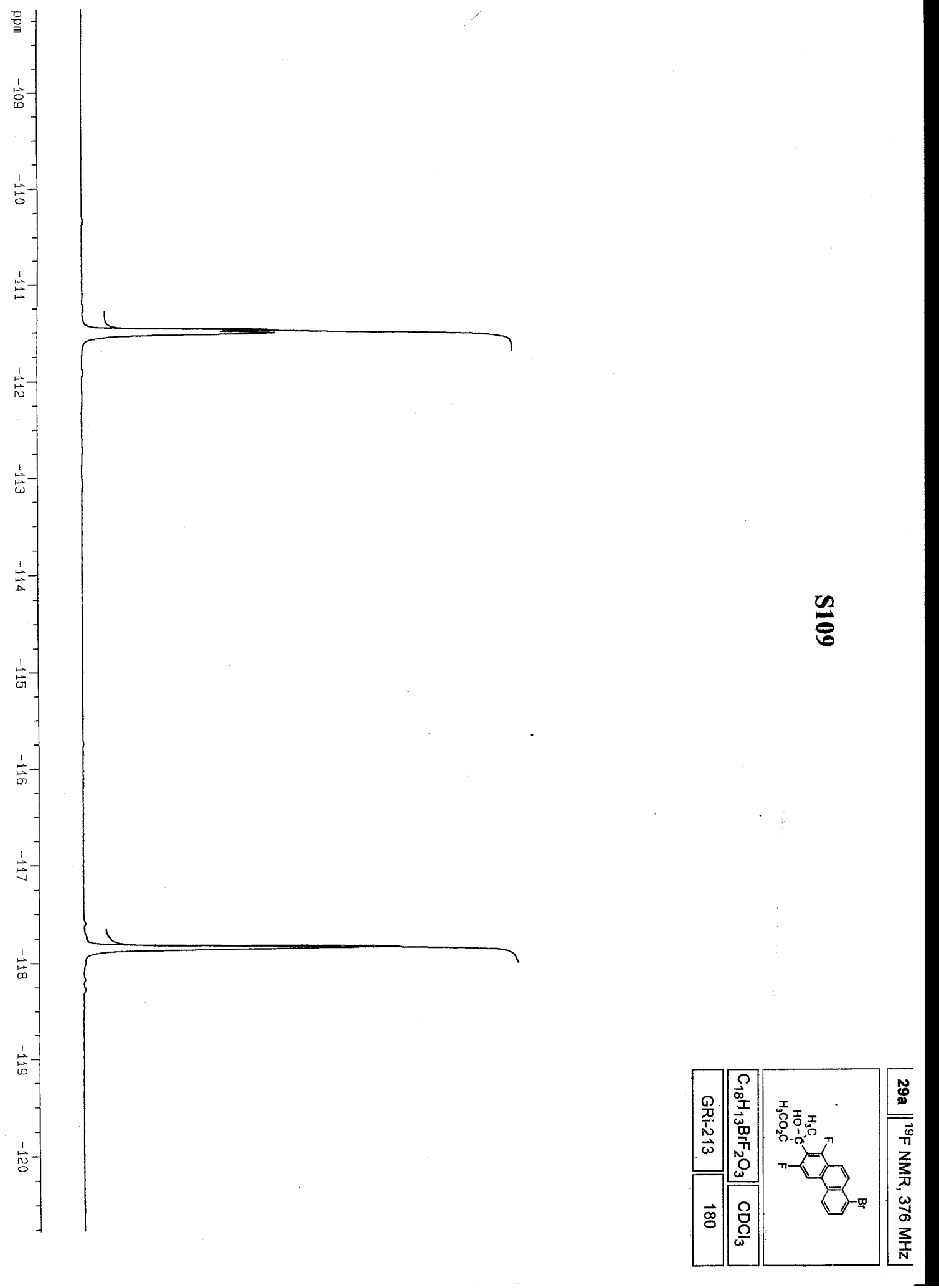

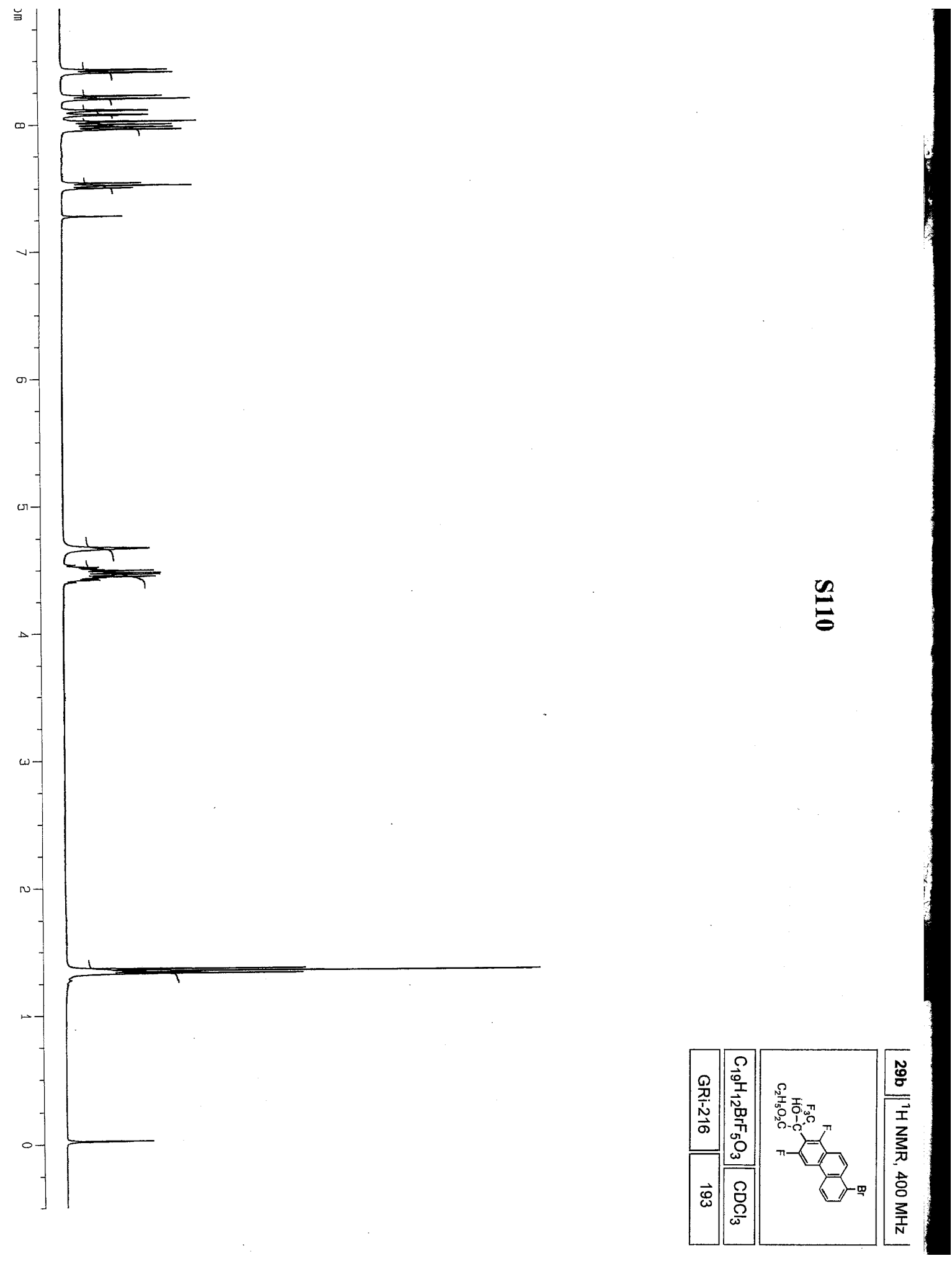

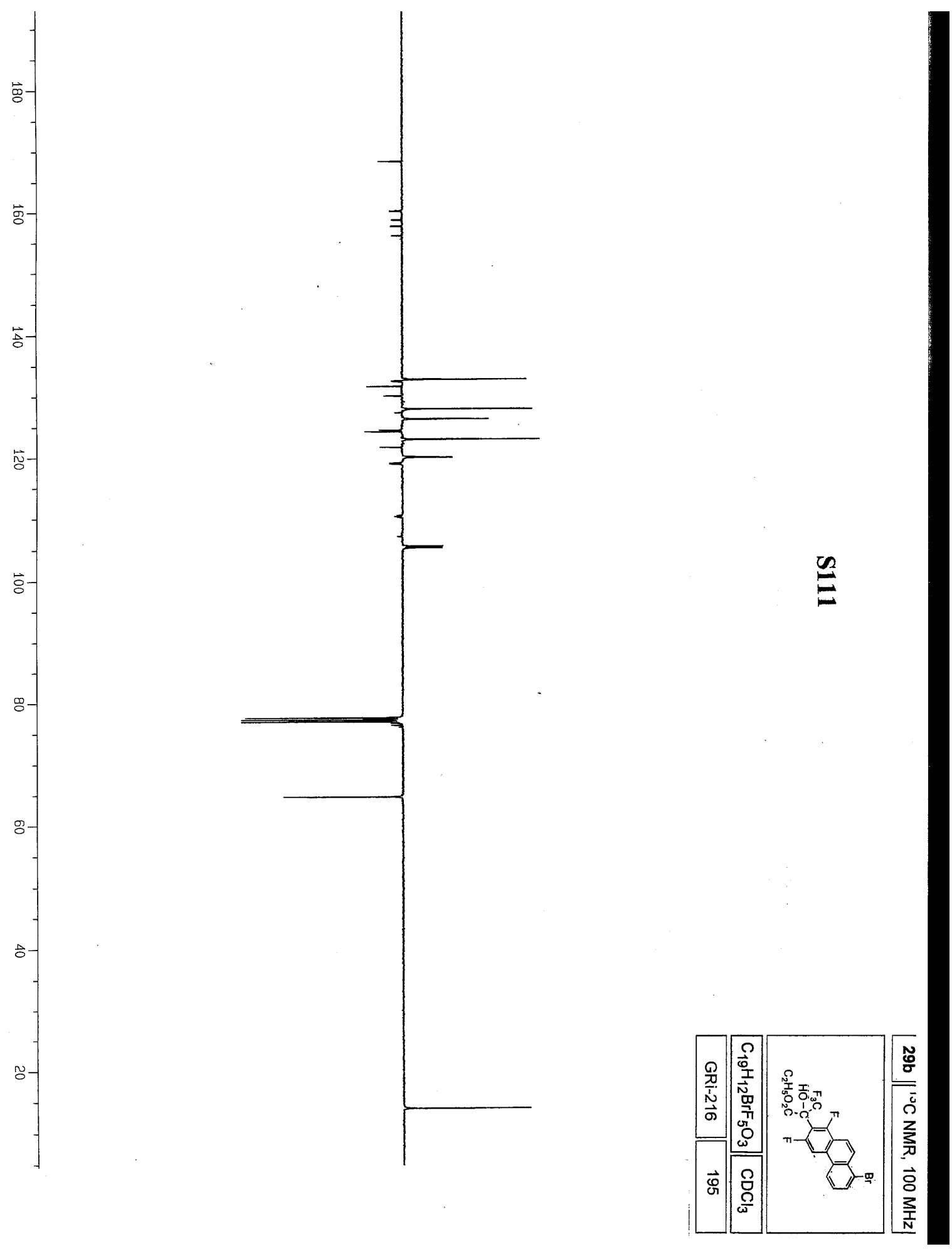


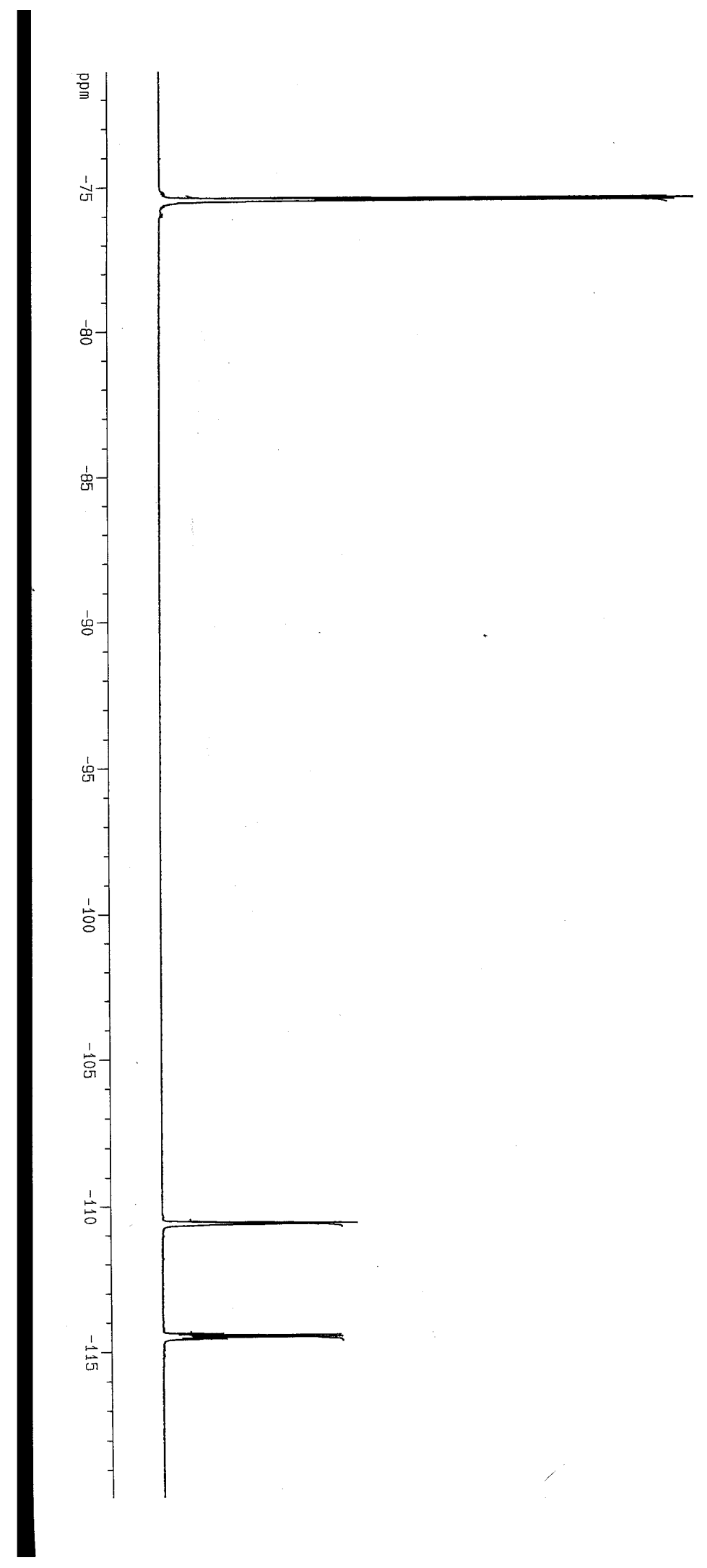

$\frac{2}{5}$

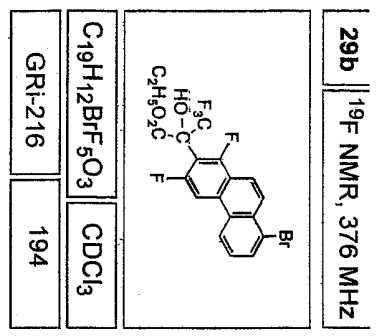




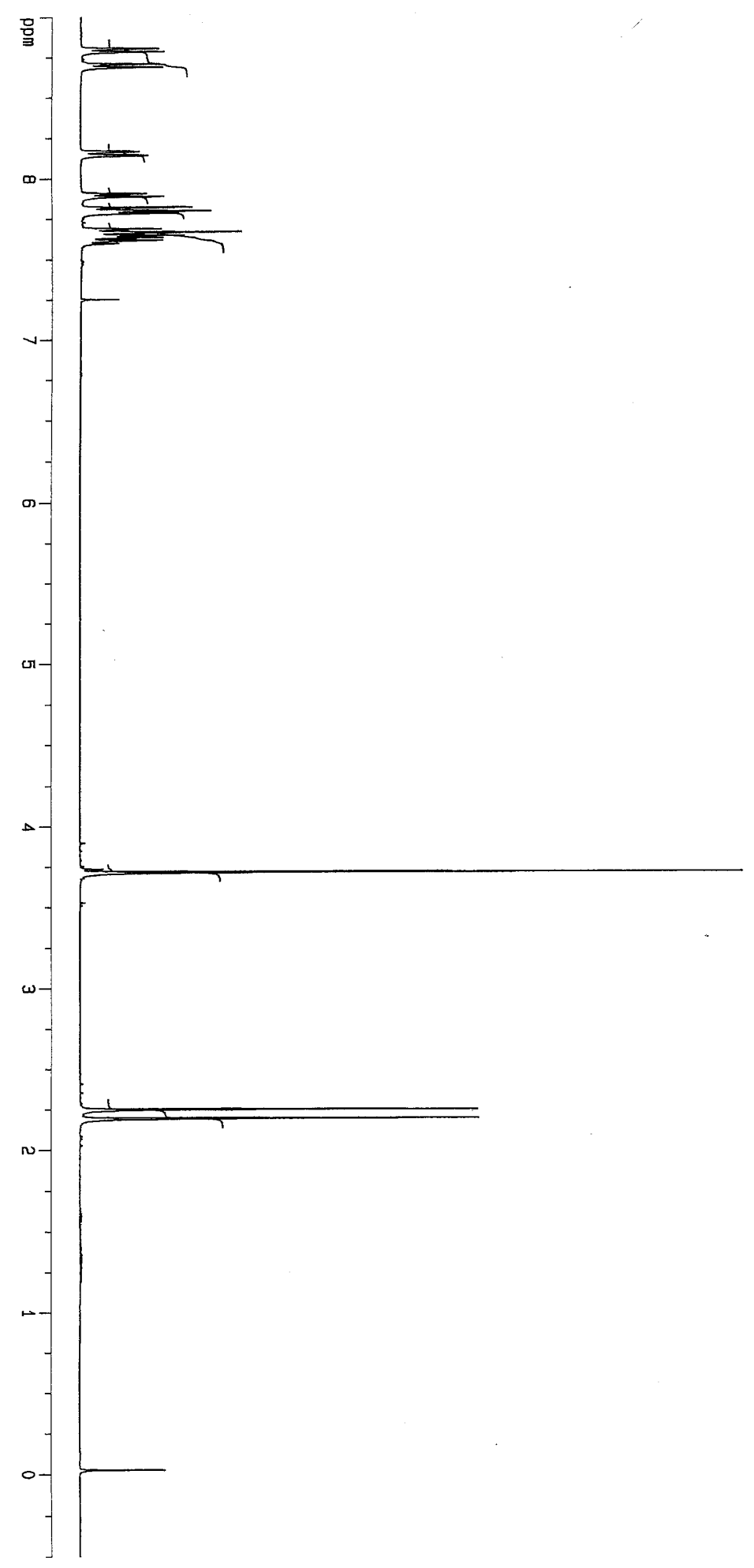

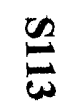

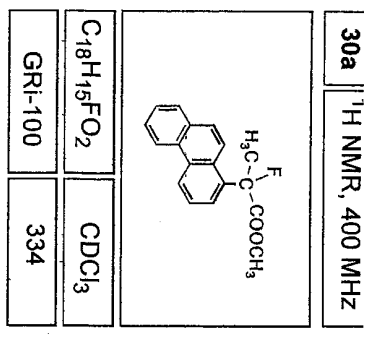




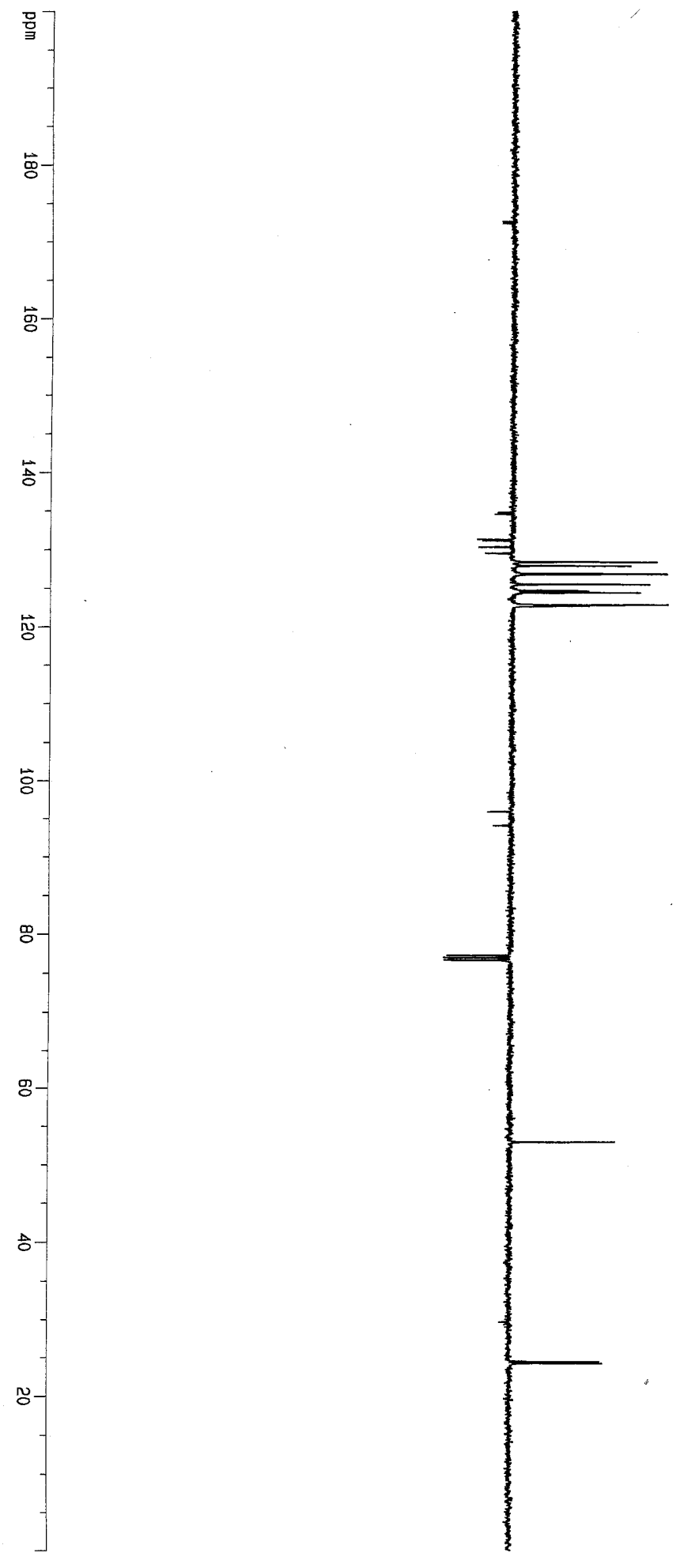

$\underline{2}$

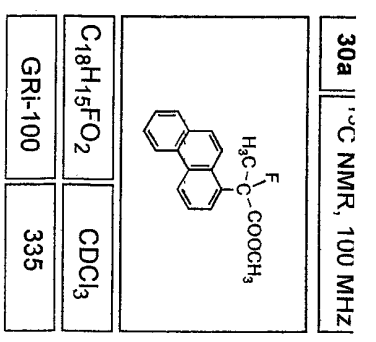



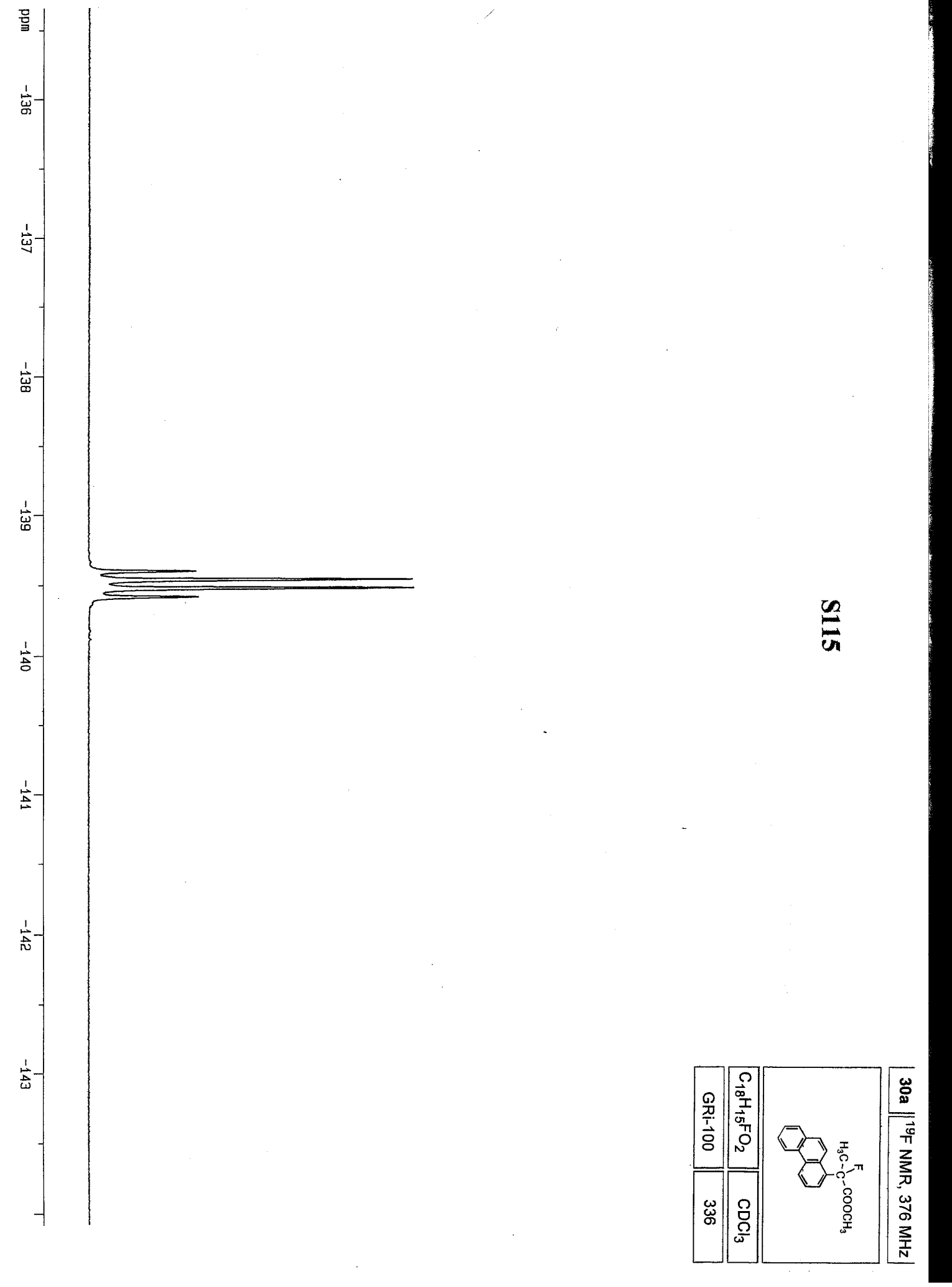


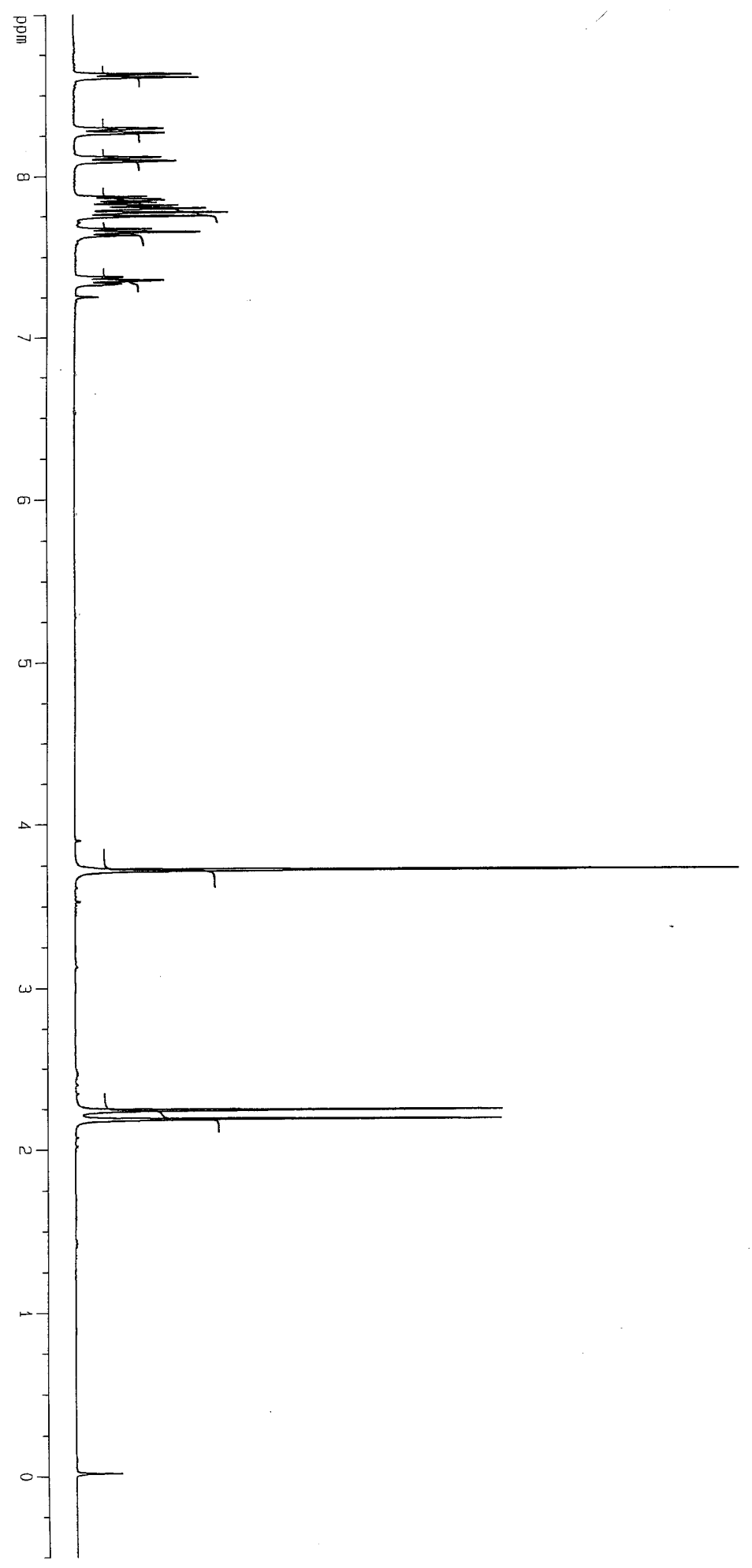

$\frac{6}{a}$

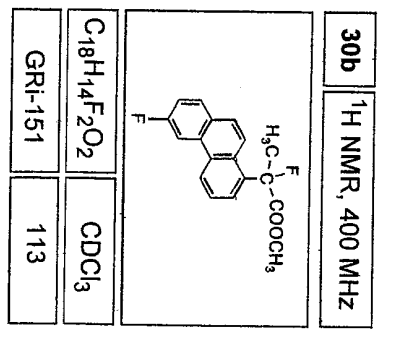



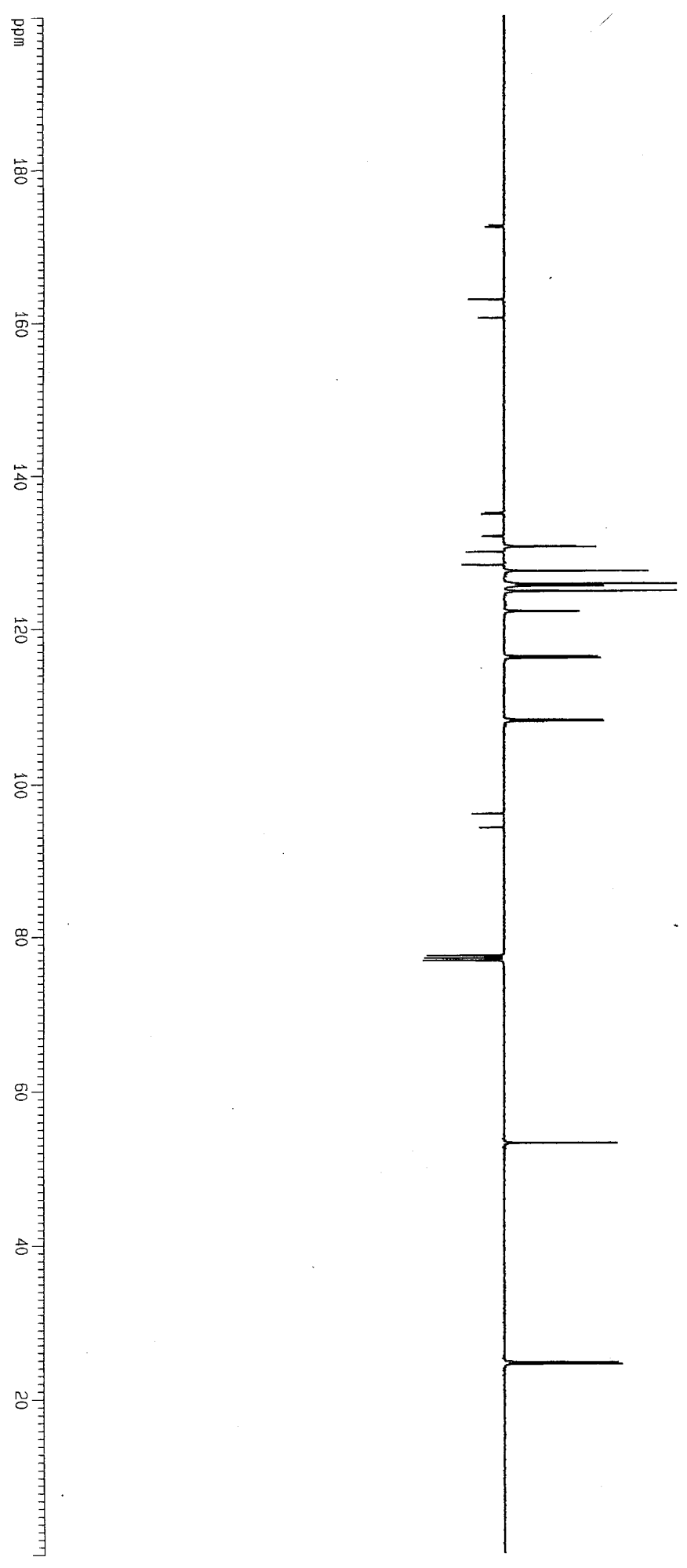

$\underset{v}{0}$

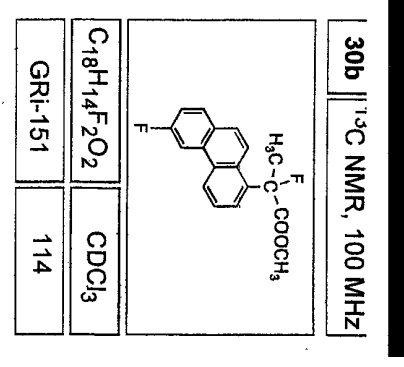



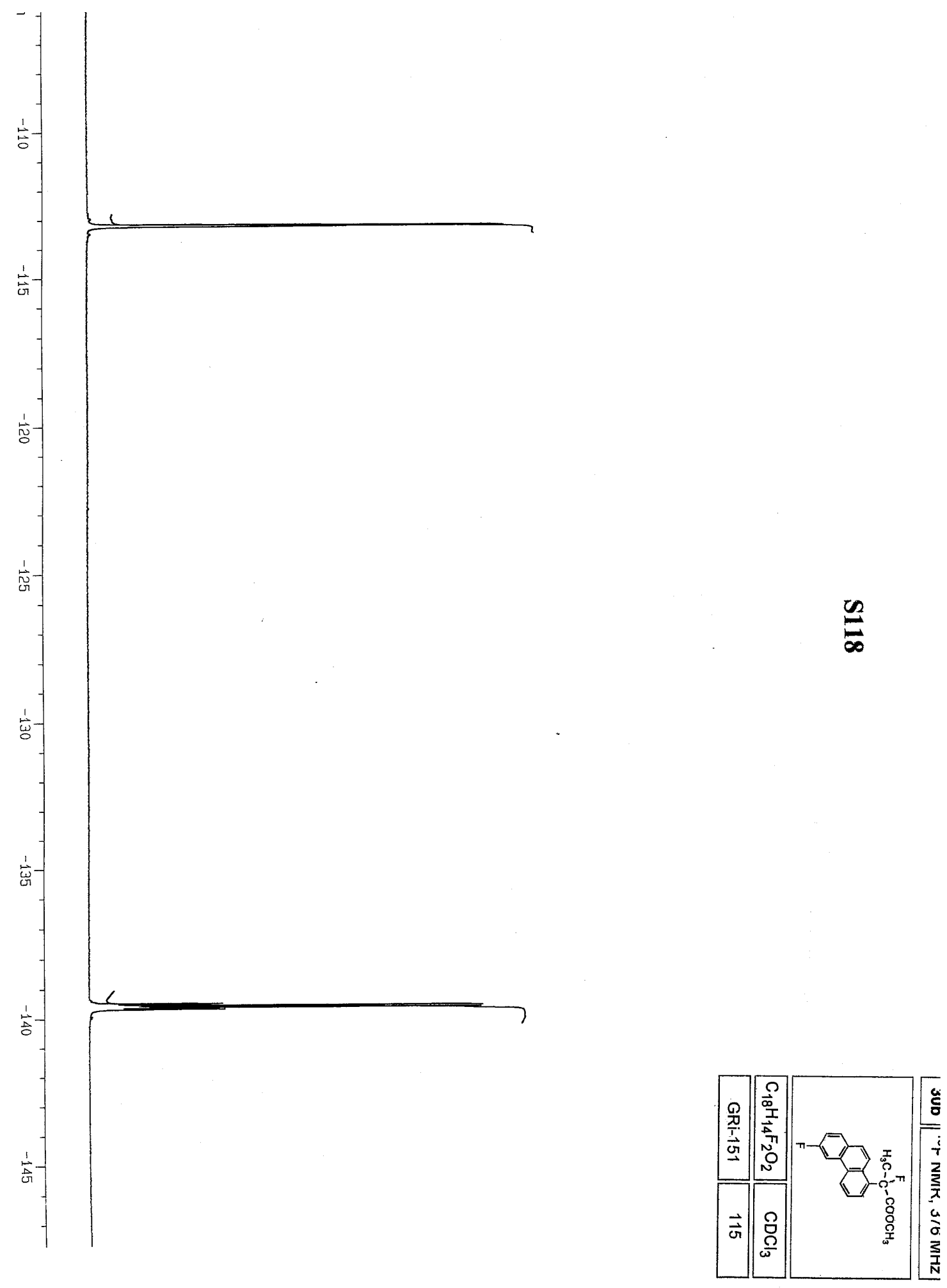

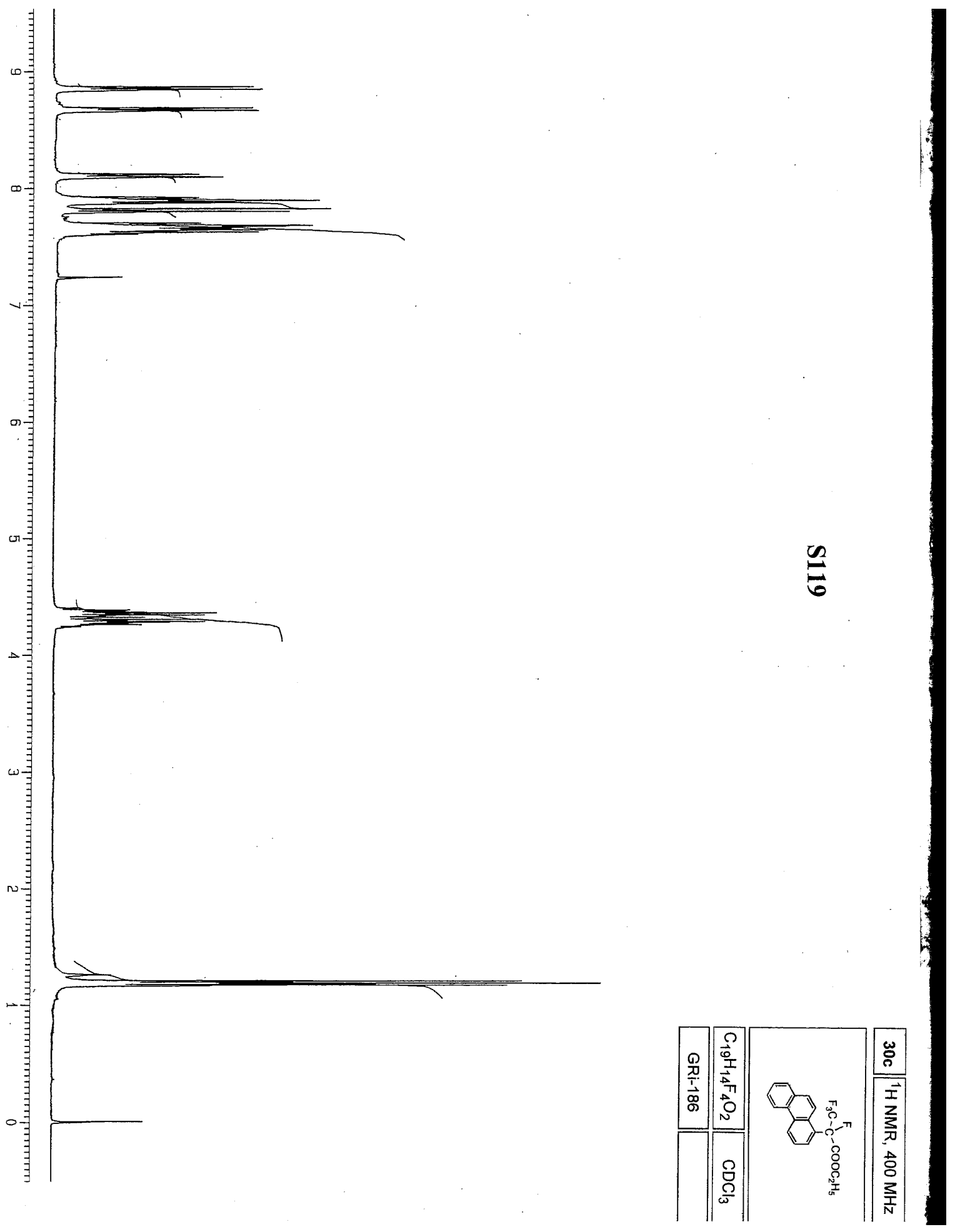


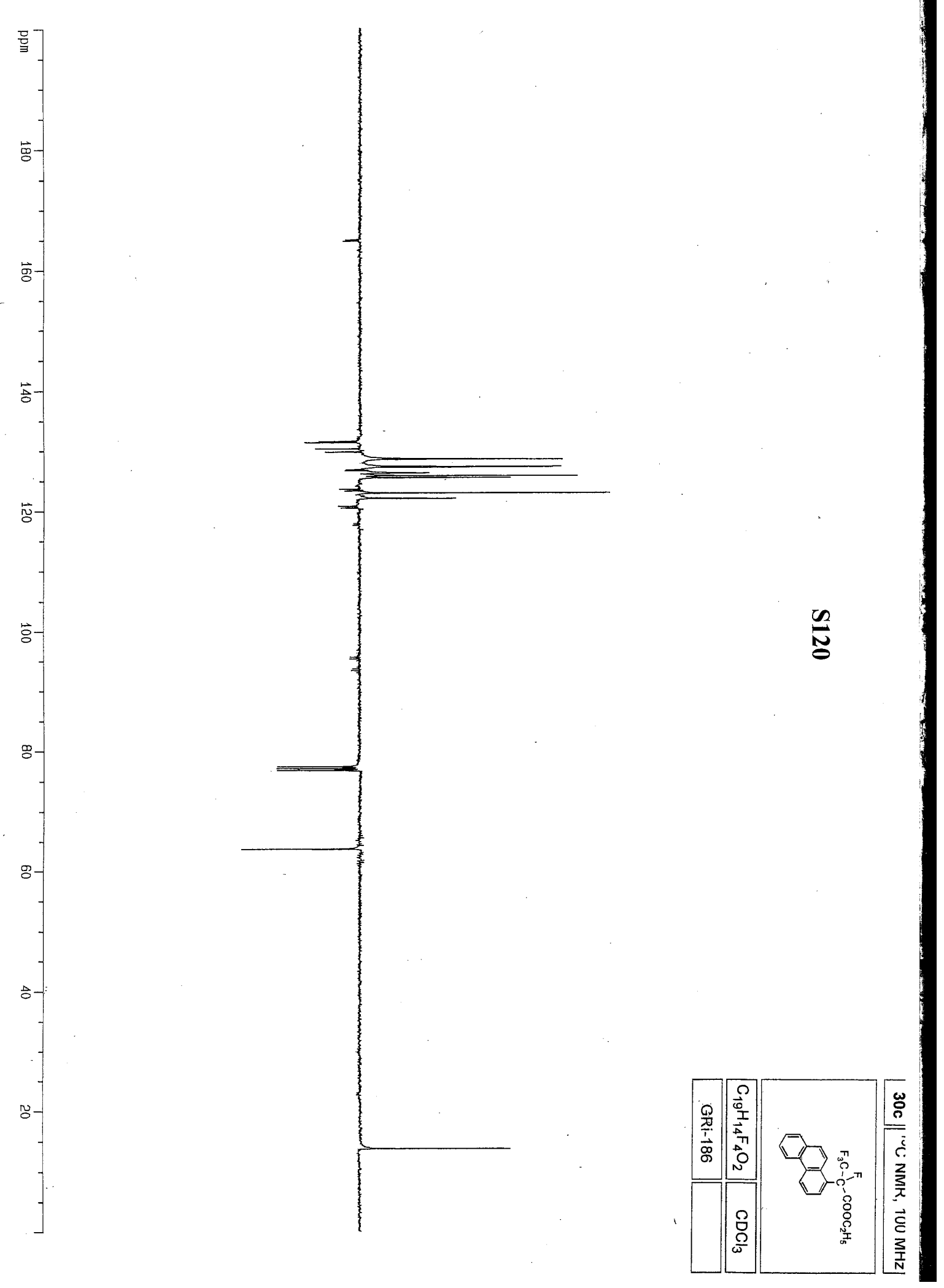



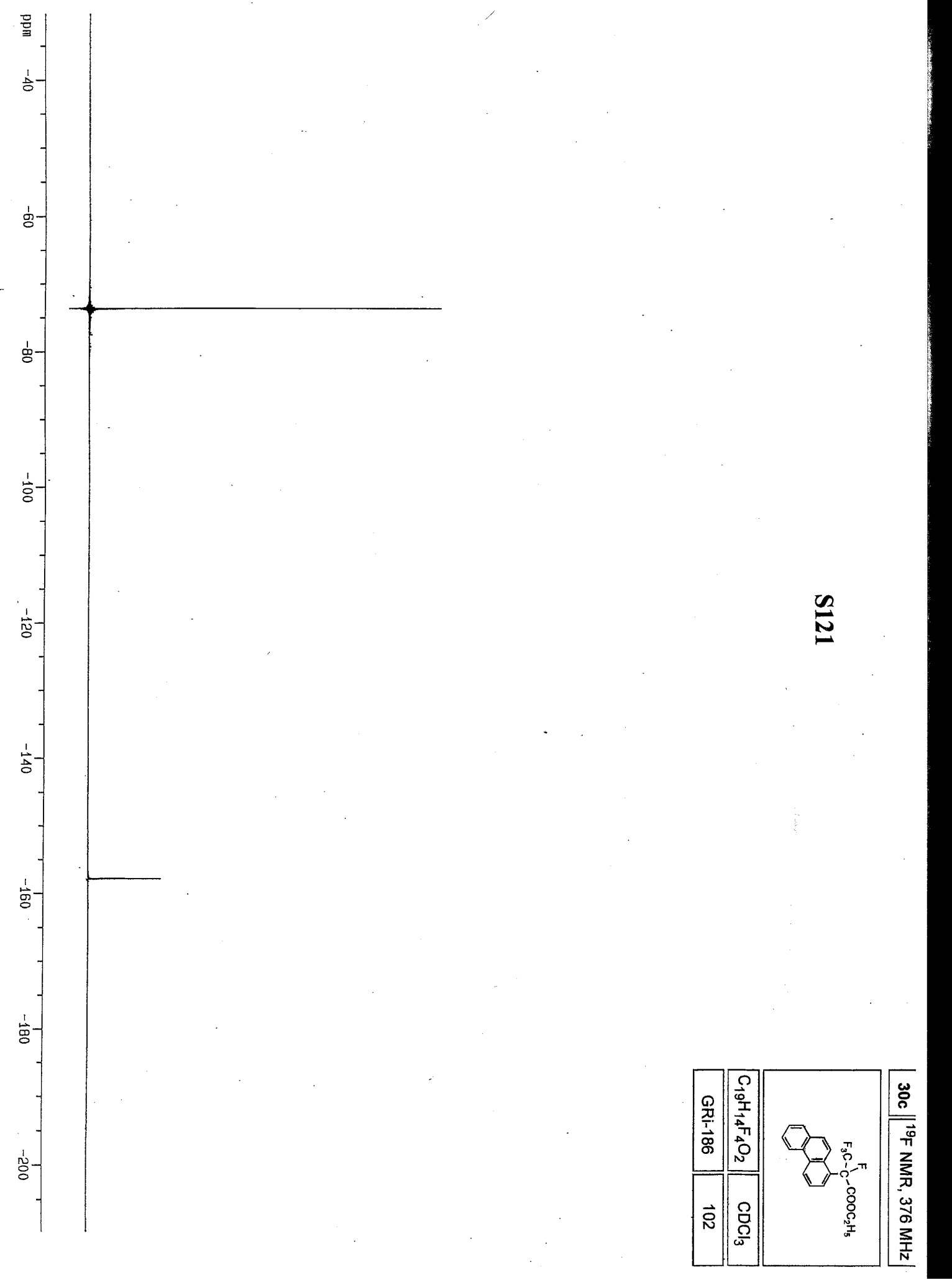


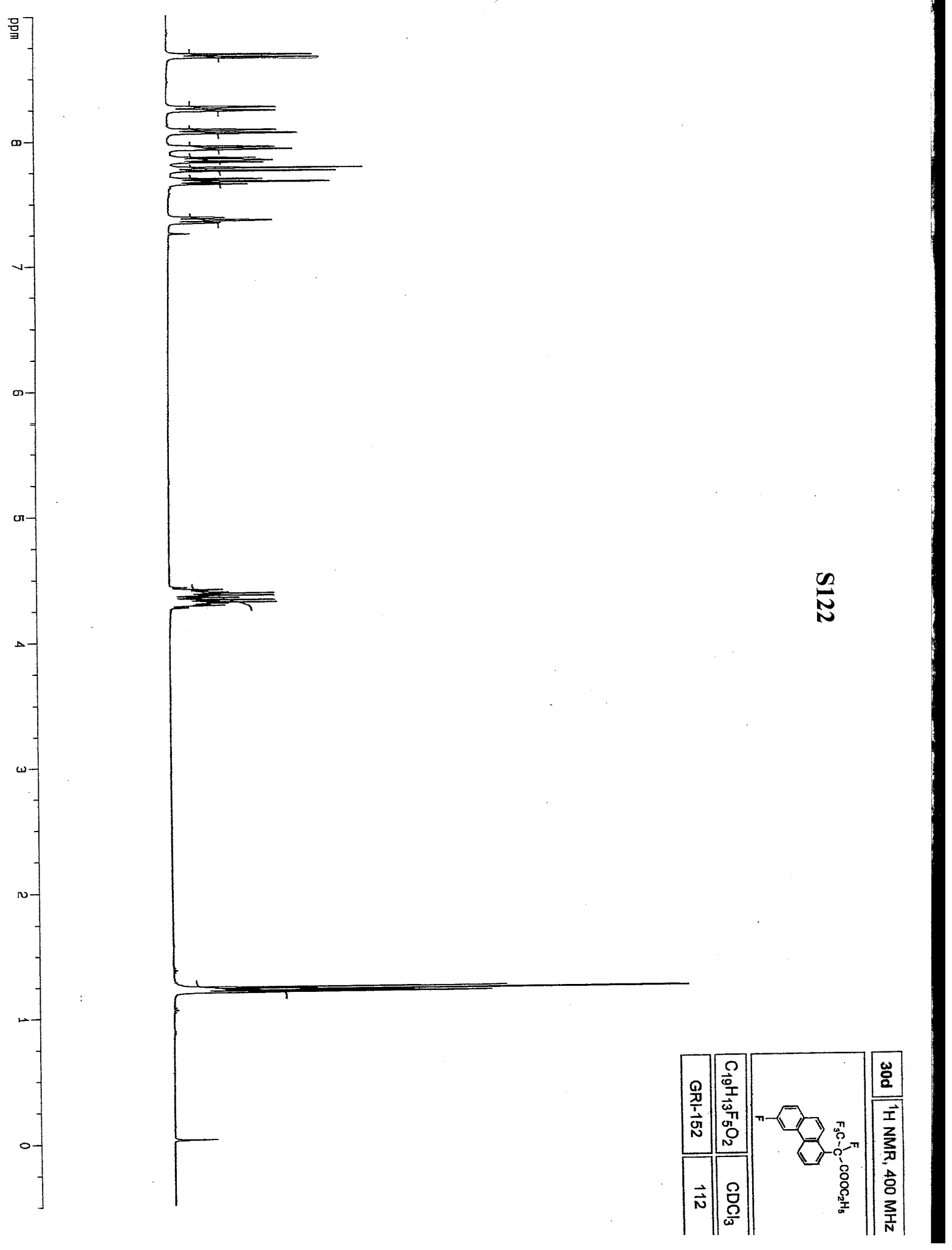



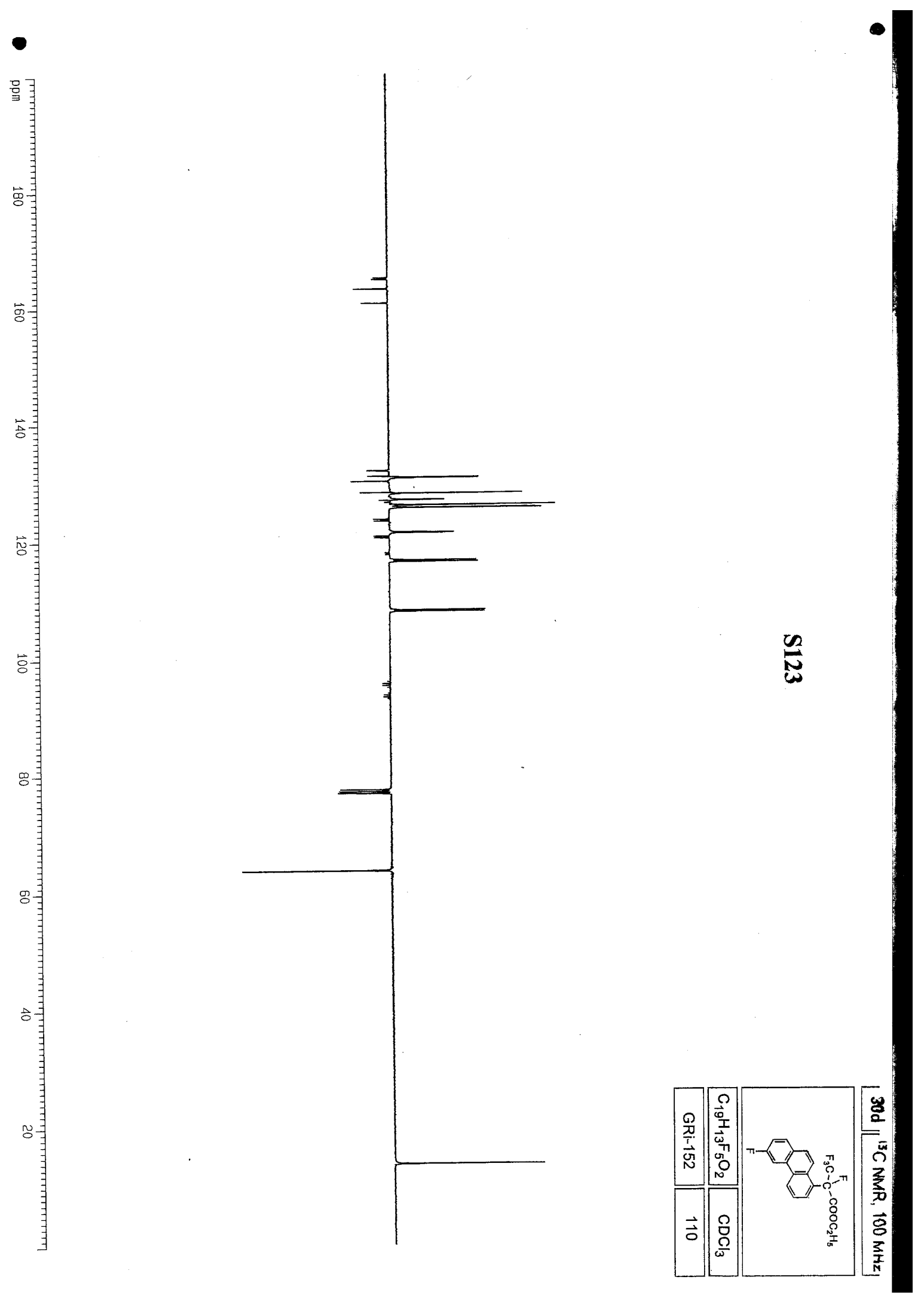

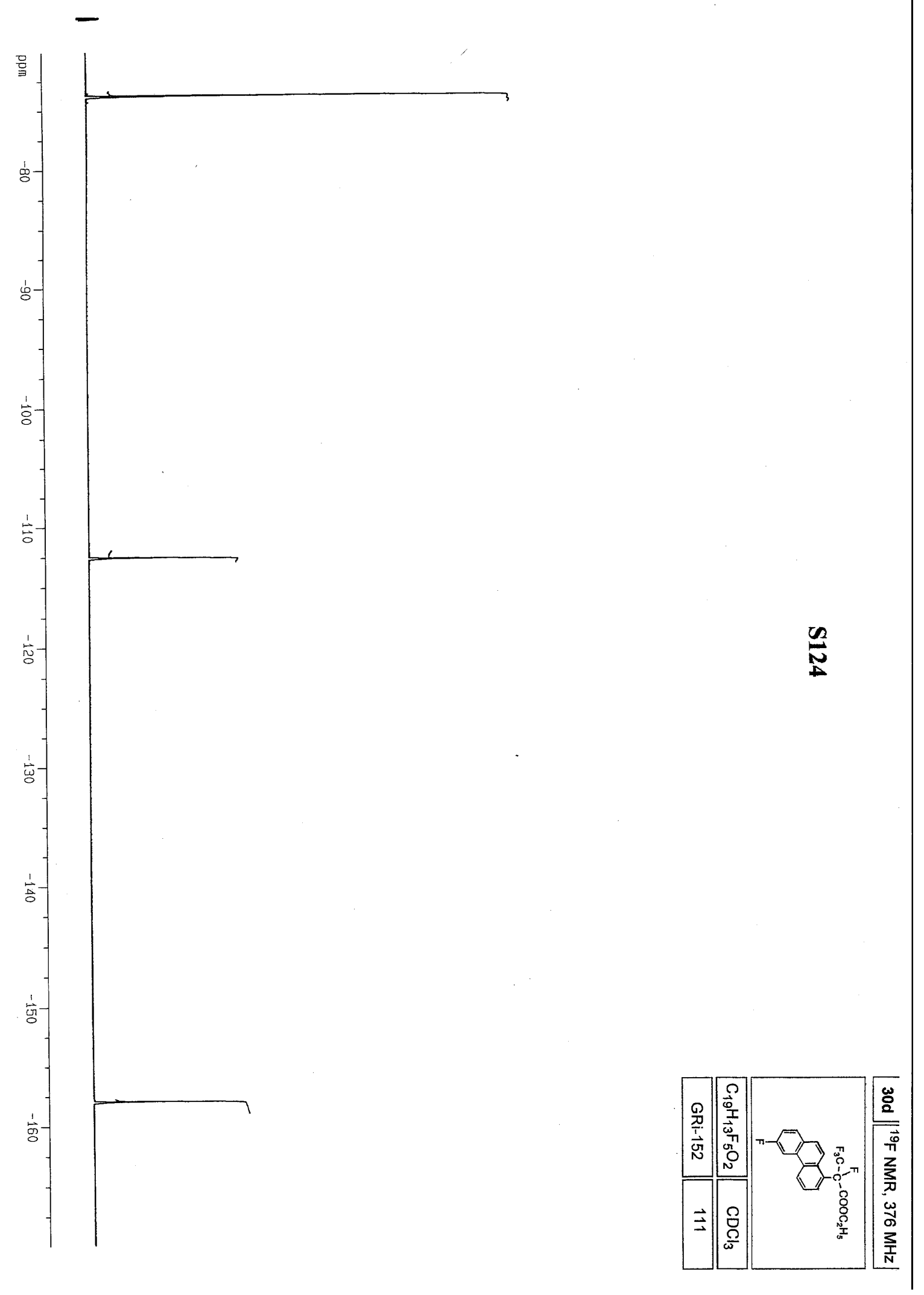

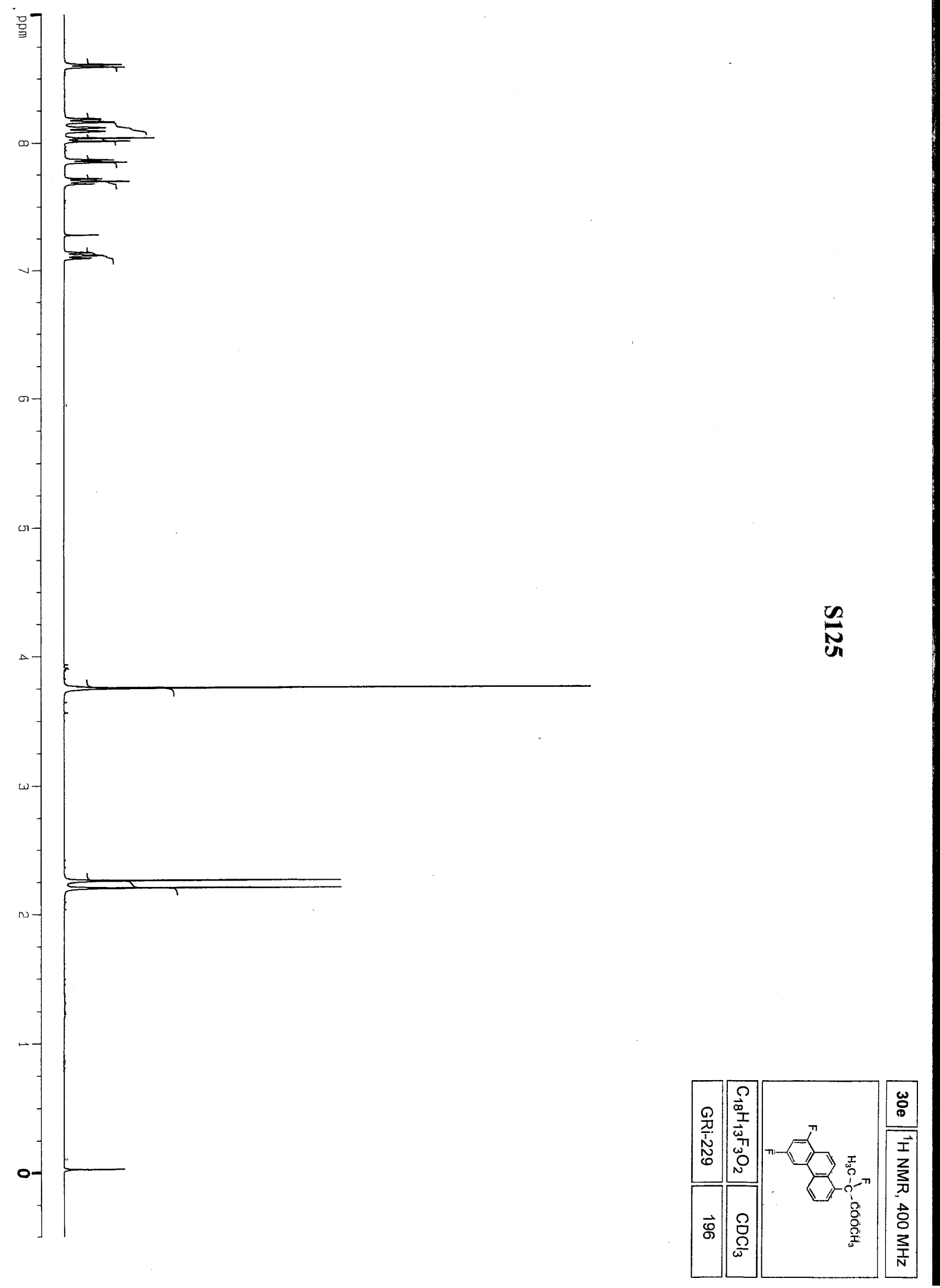

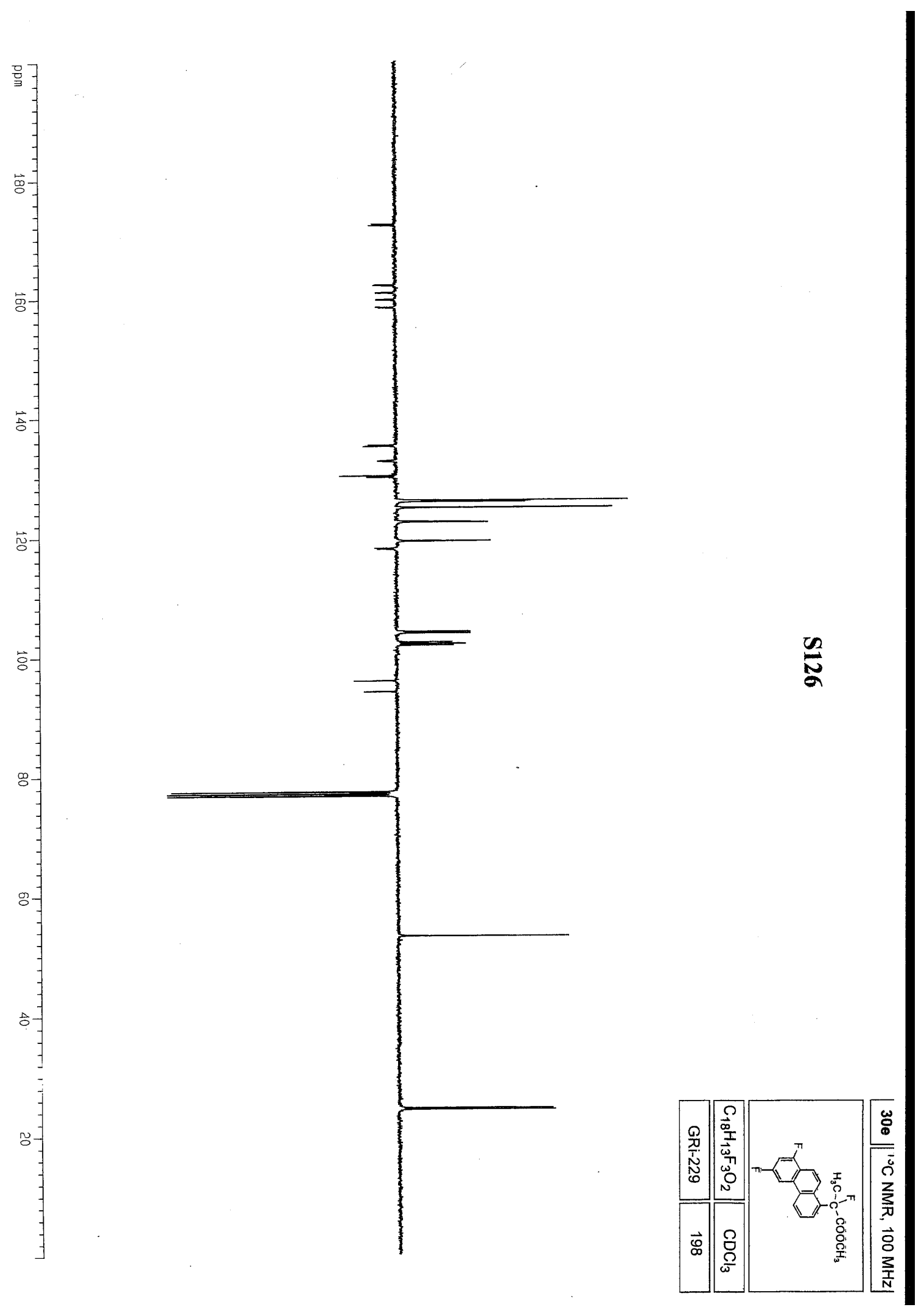


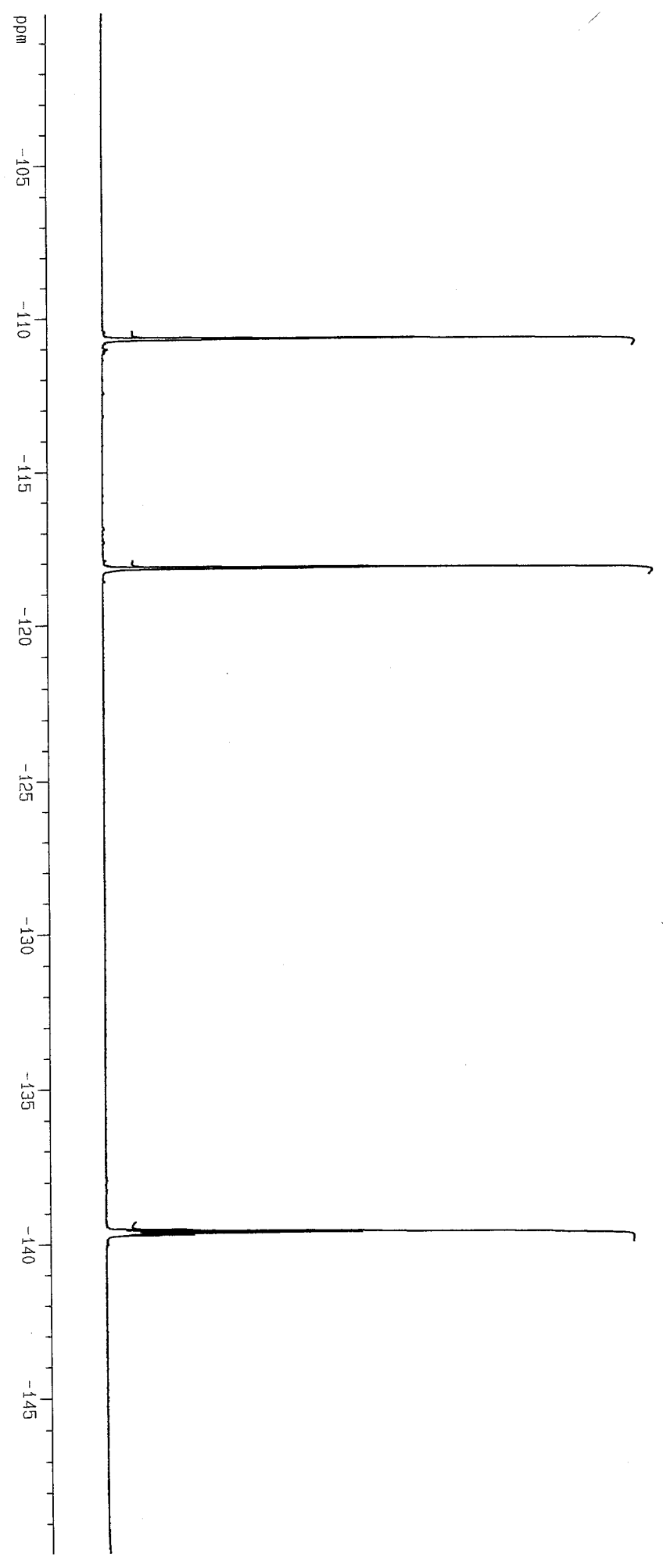

$\frac{2}{3}$

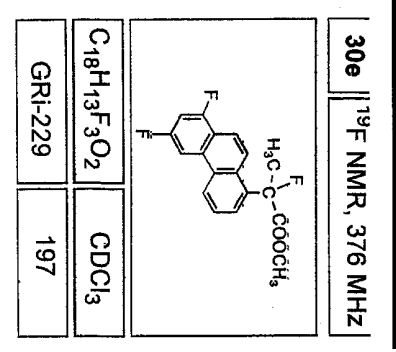




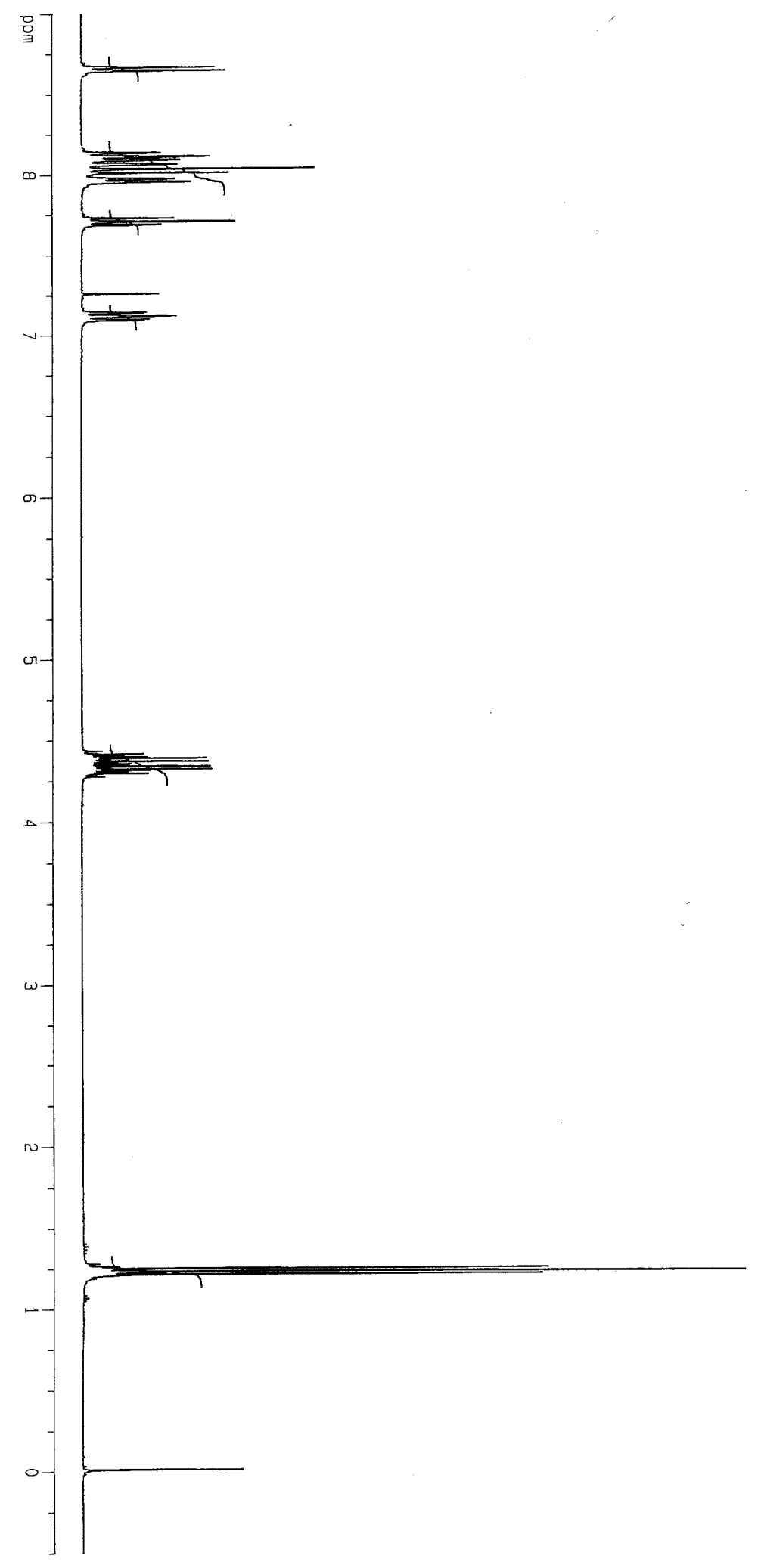

$\frac{\infty}{\infty}$

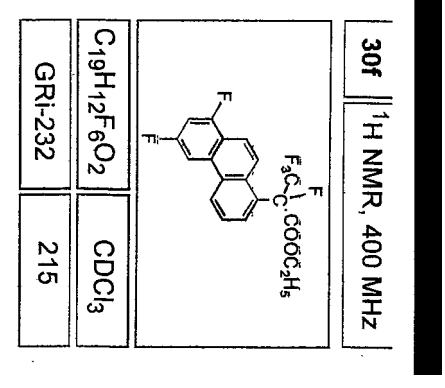



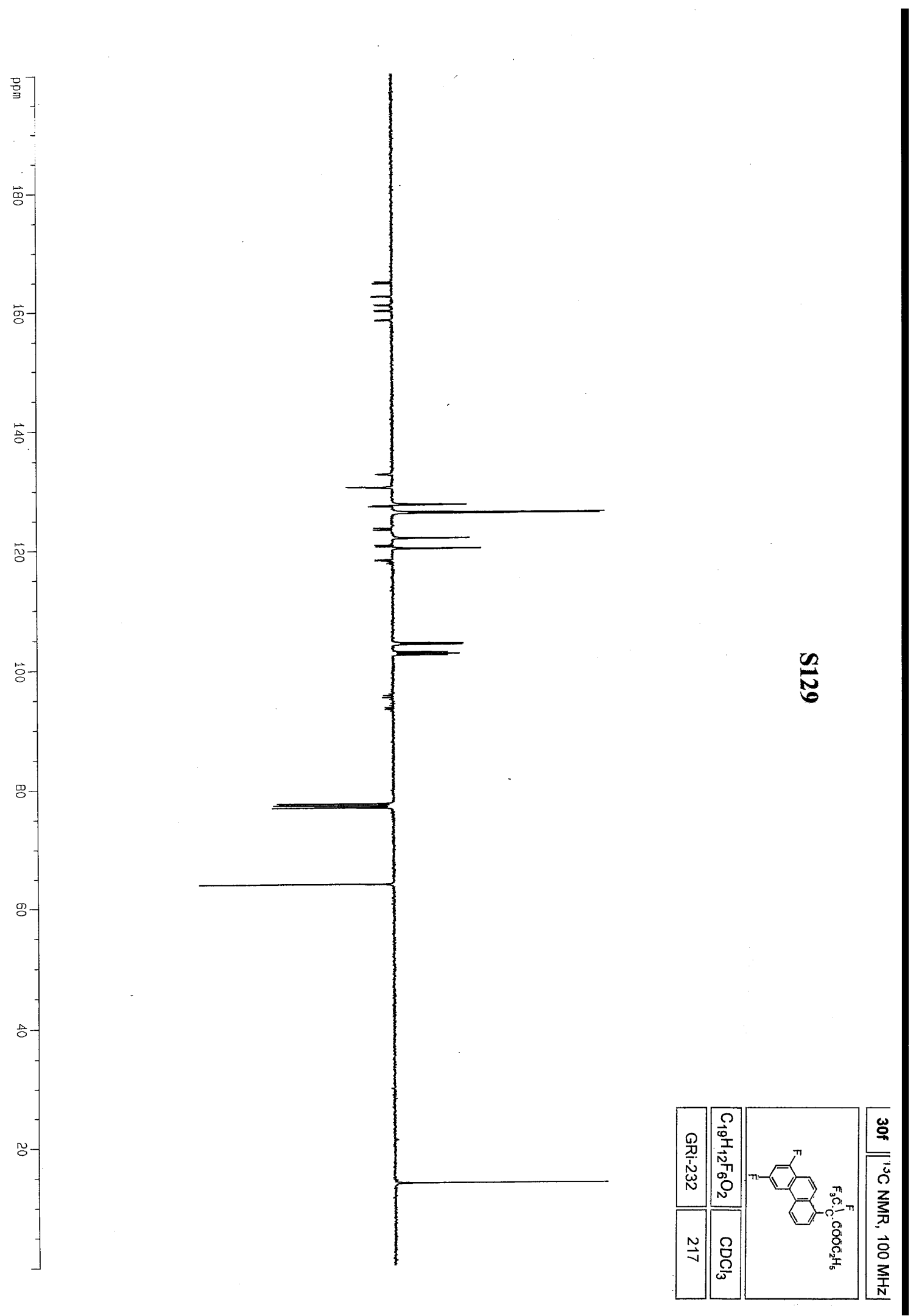


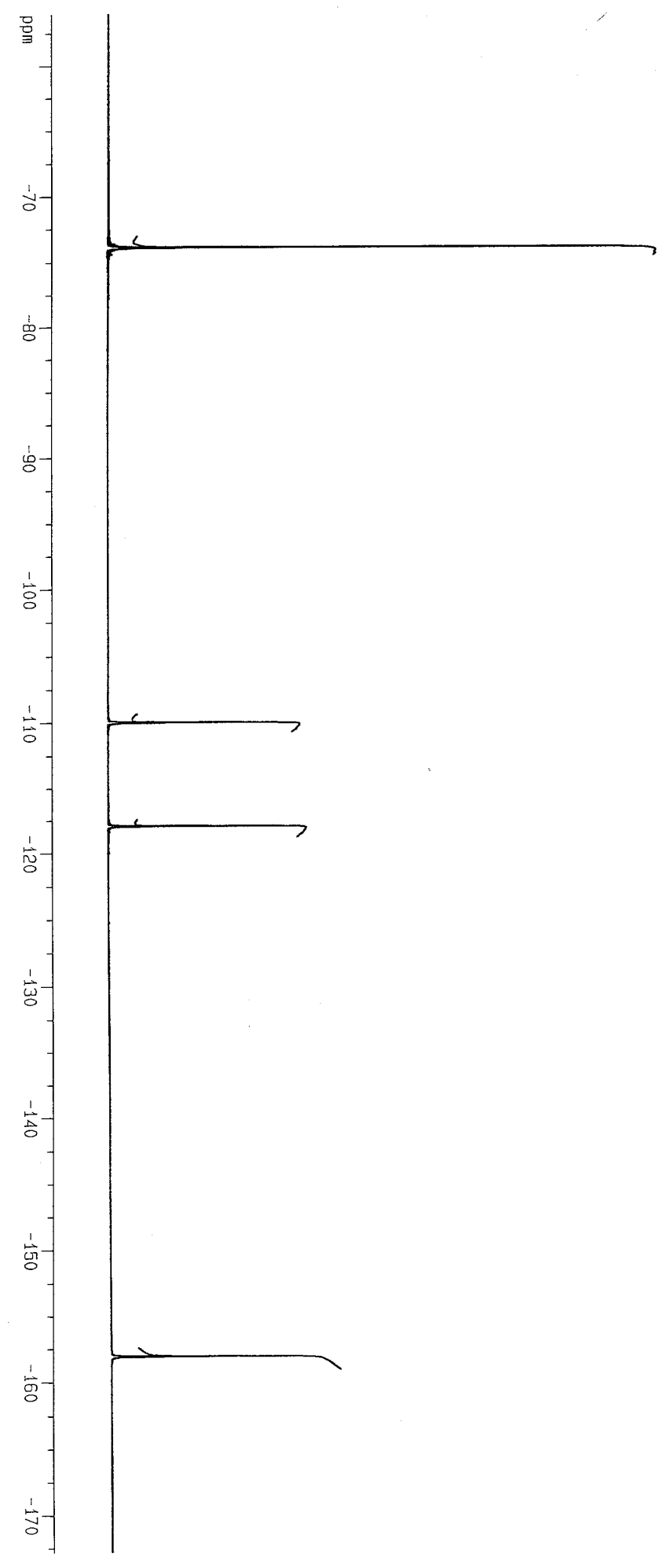

$\stackrel{0}{0}$

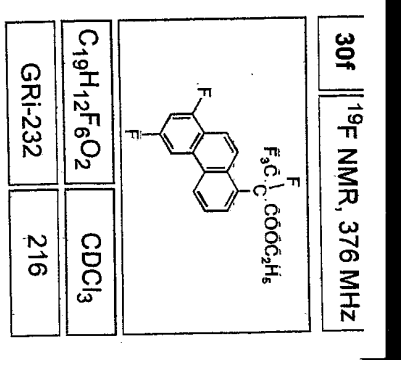




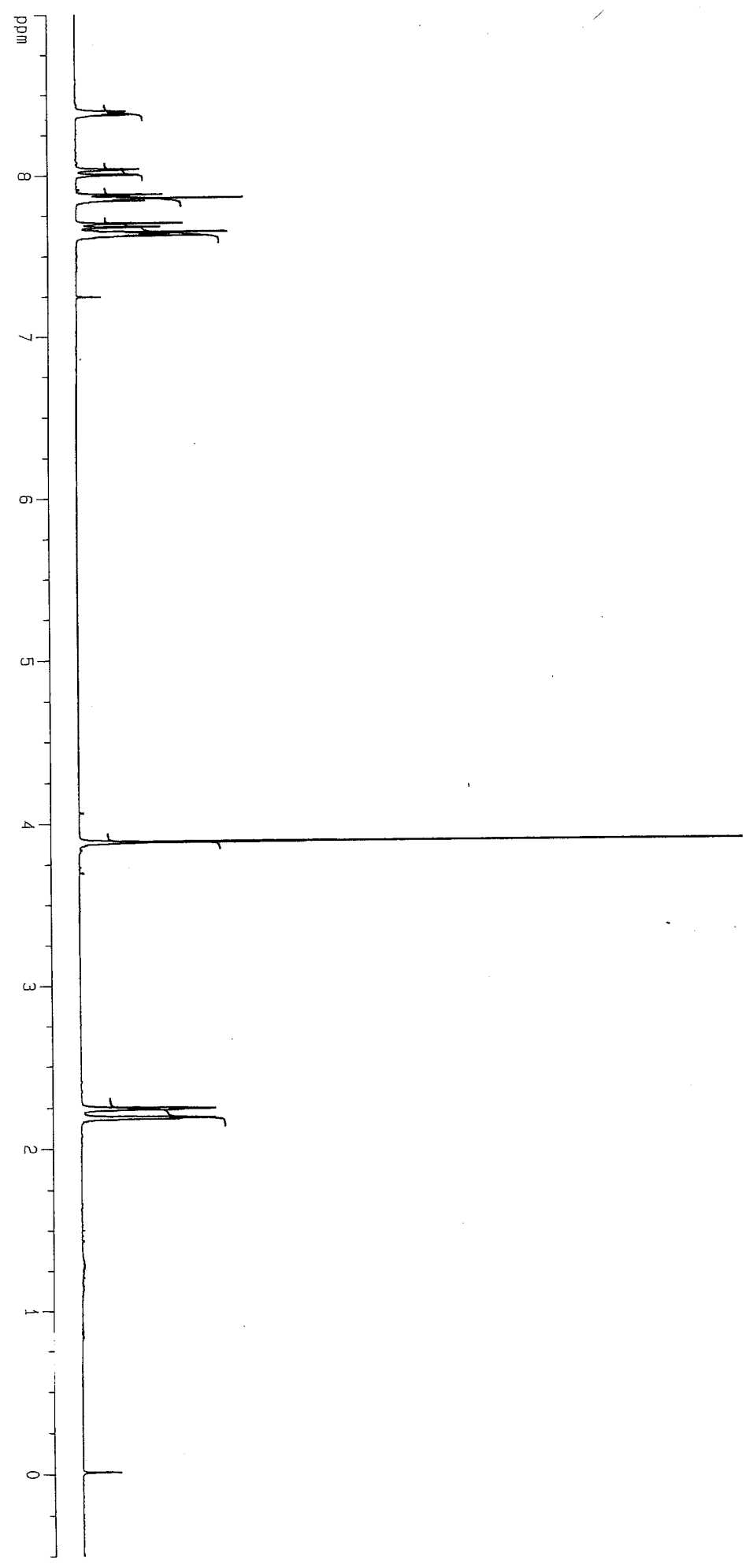

$\stackrel{\omega}{\omega}$

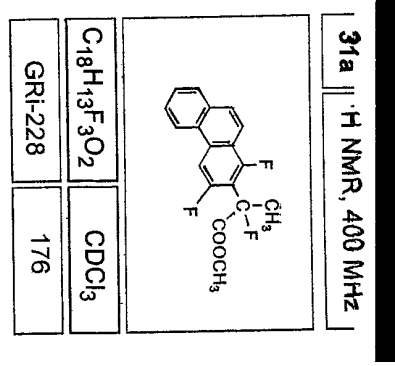



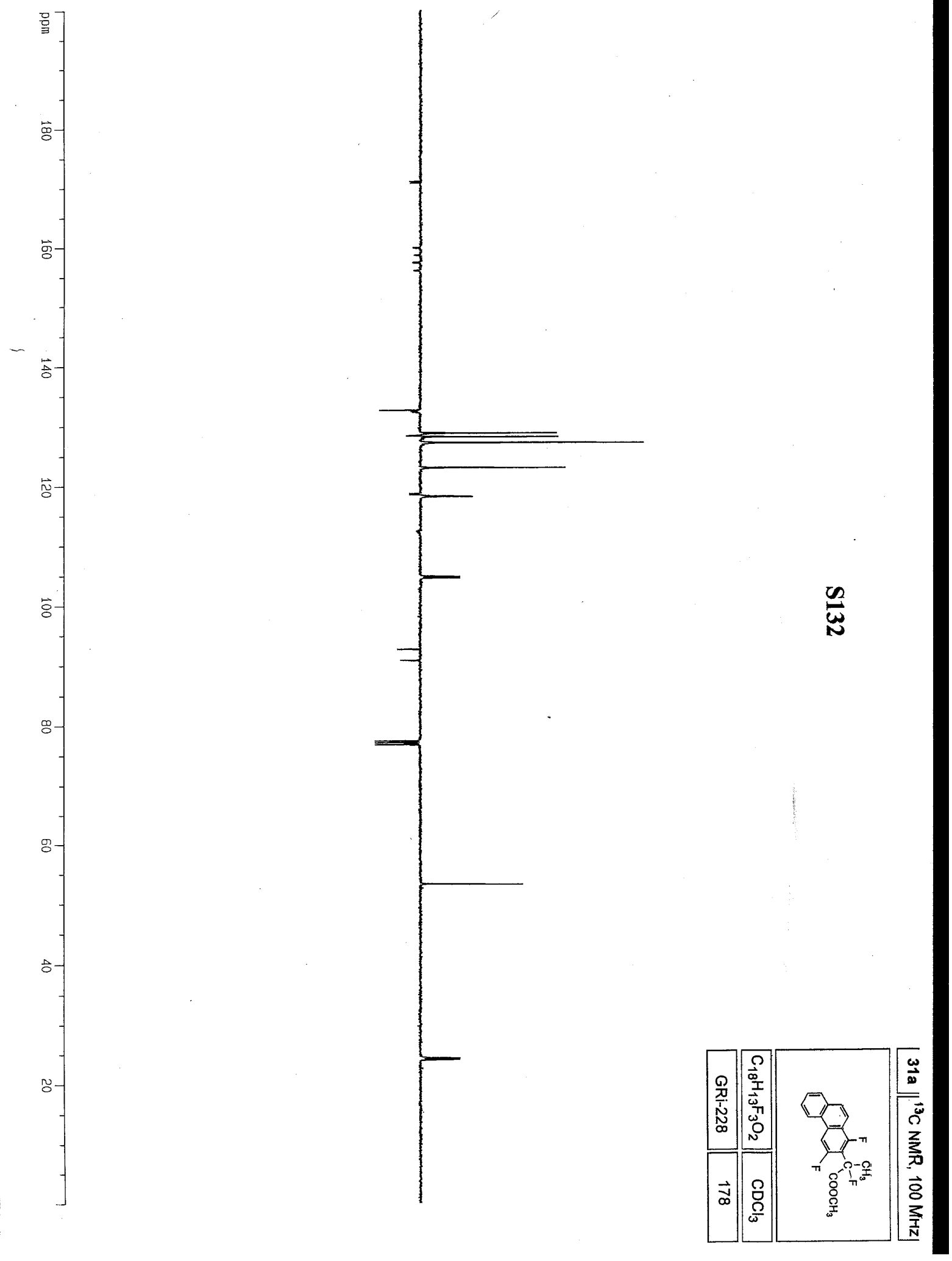

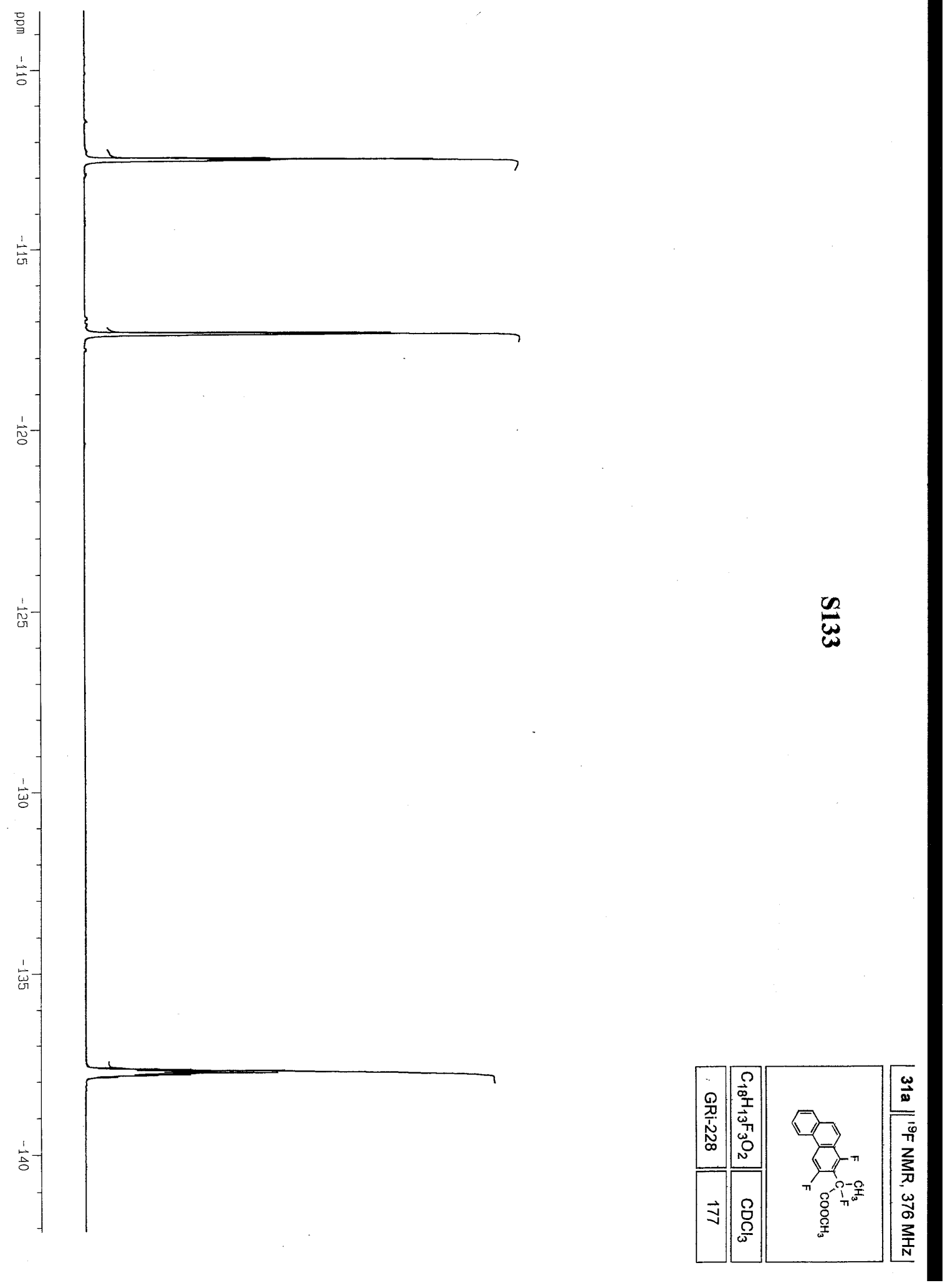


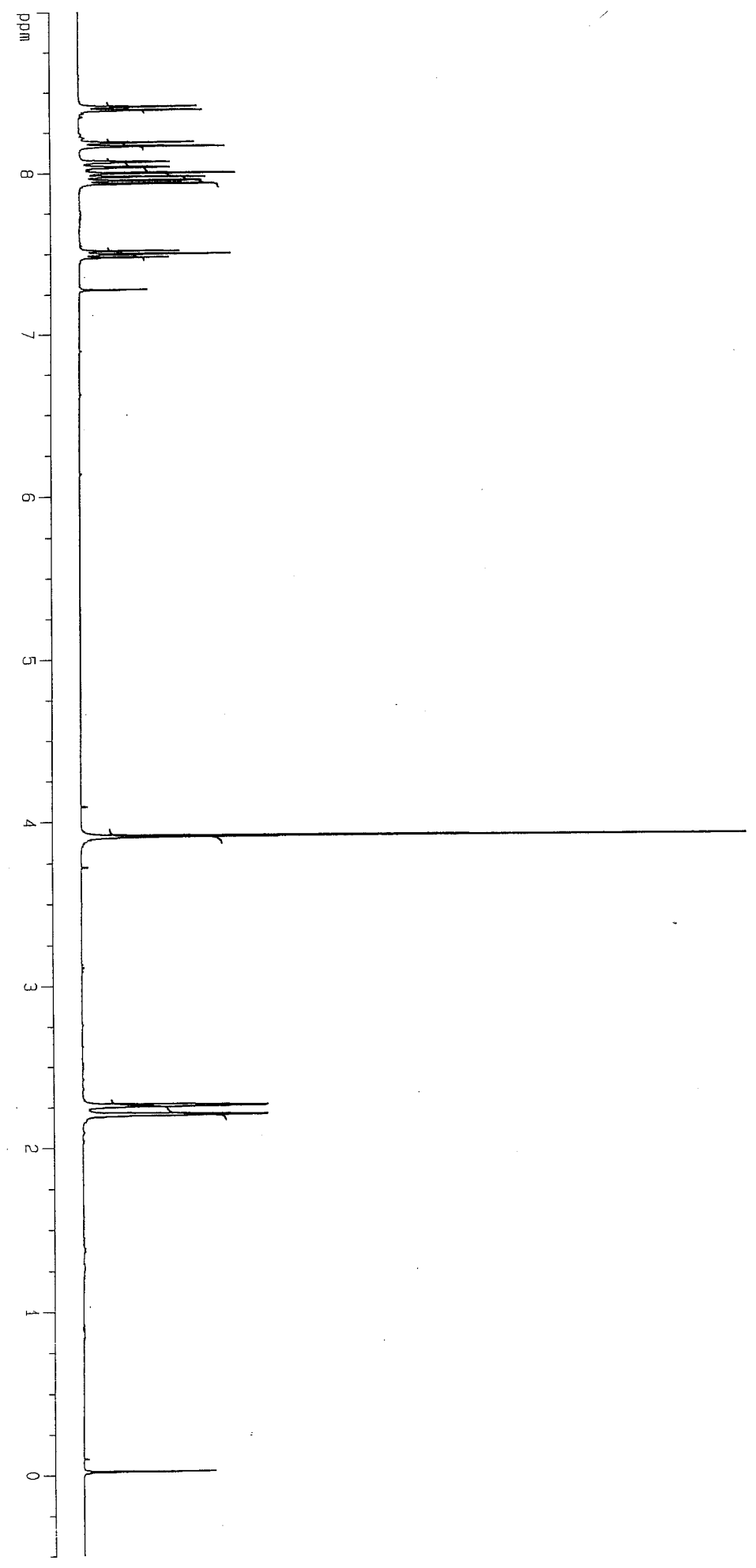

$\infty_{\infty}^{\infty}$

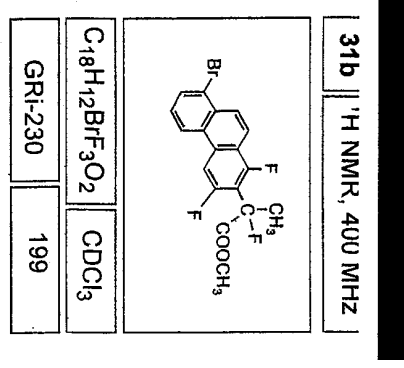



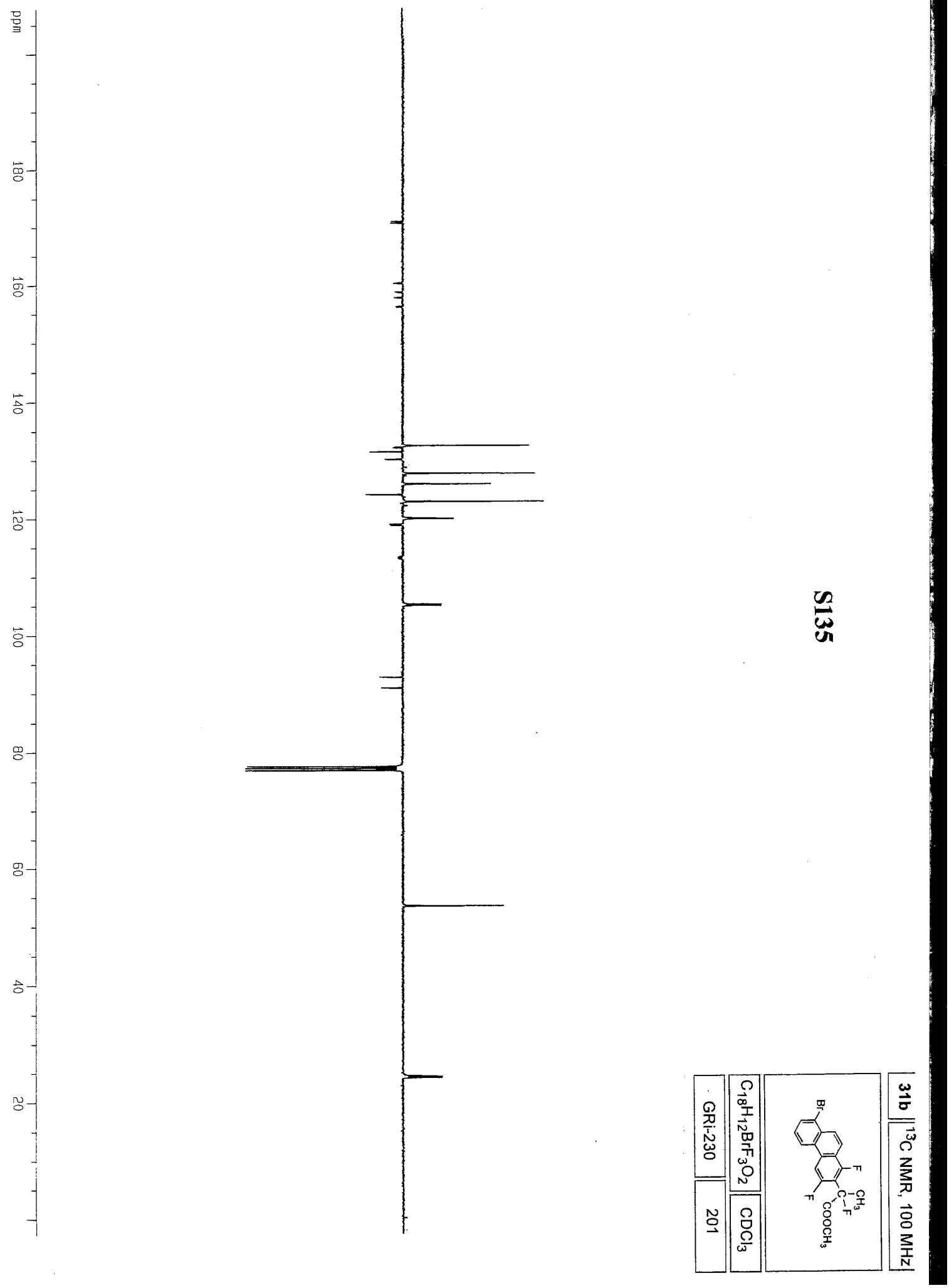

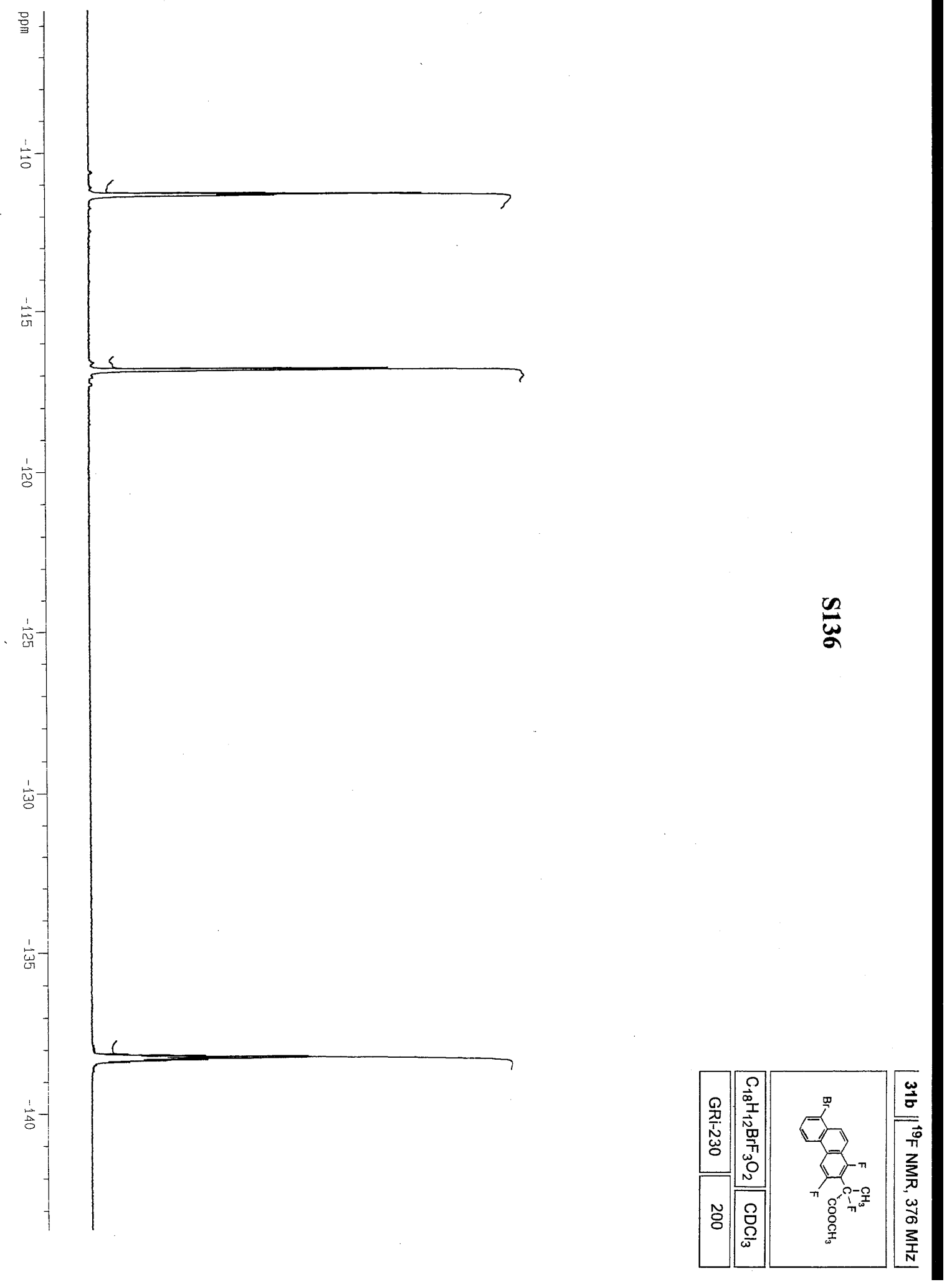


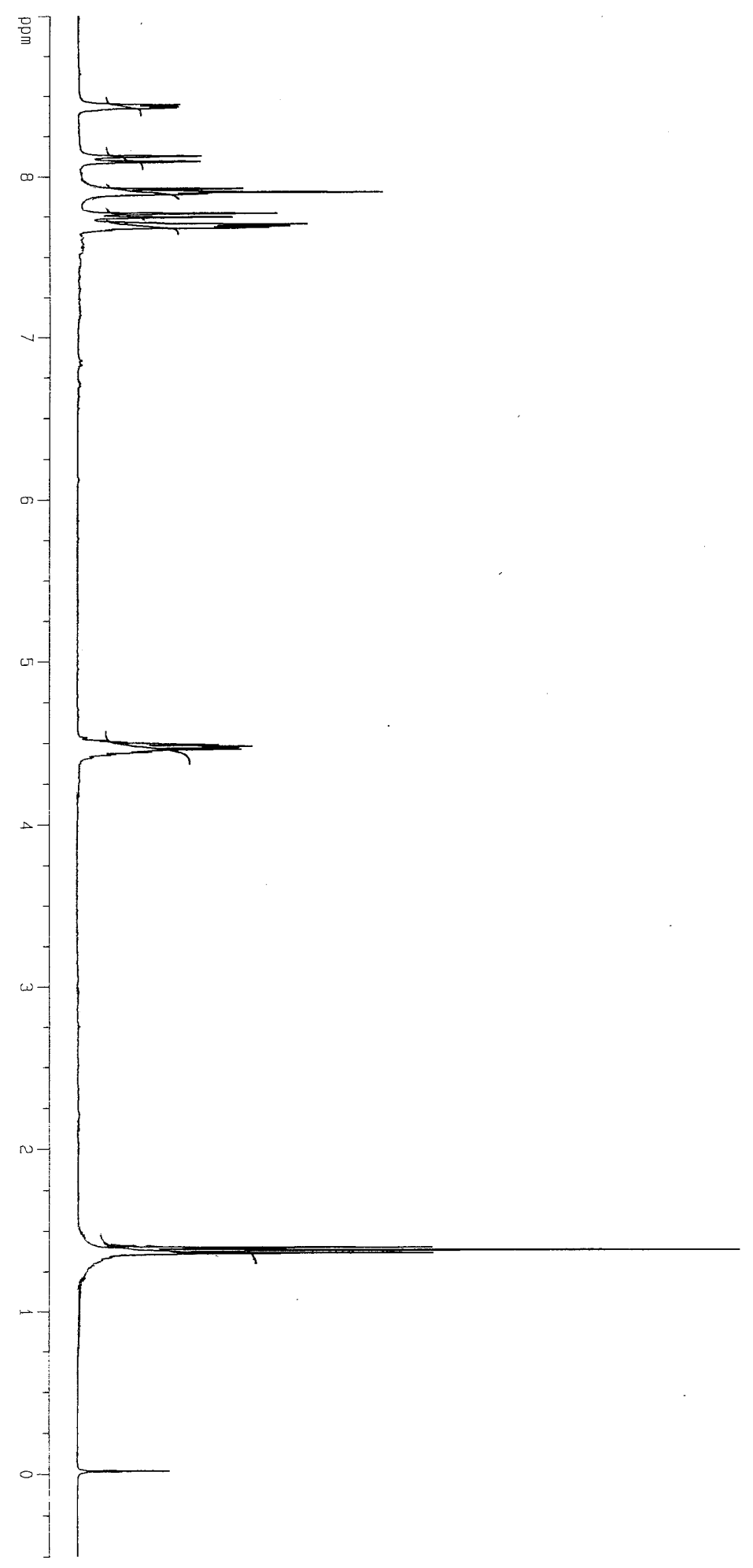

岕

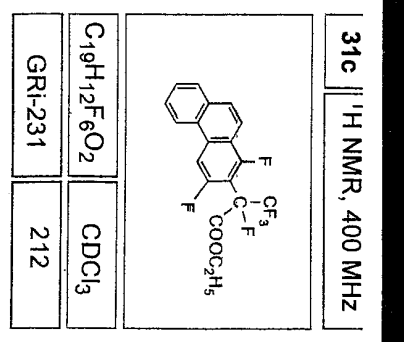



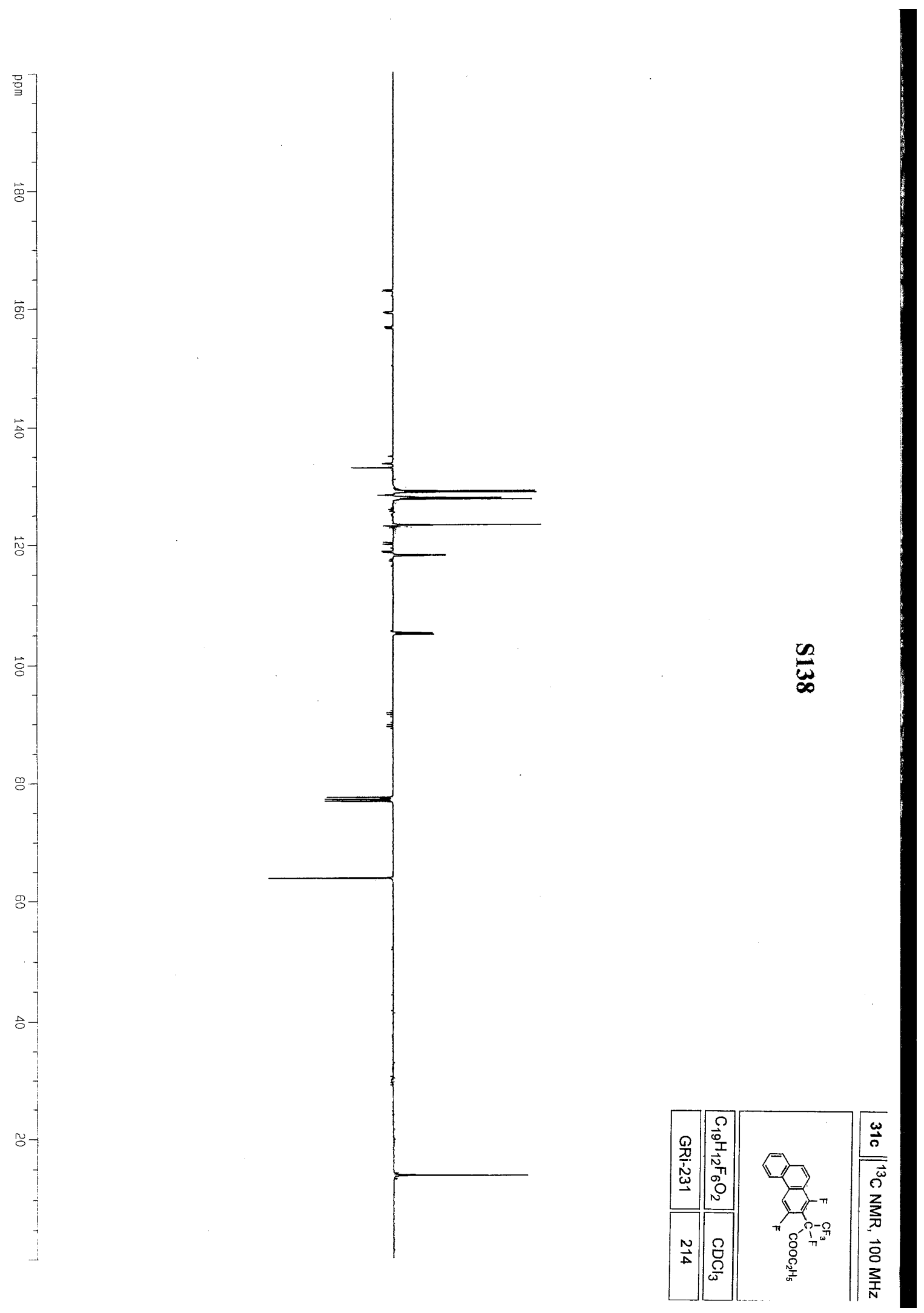


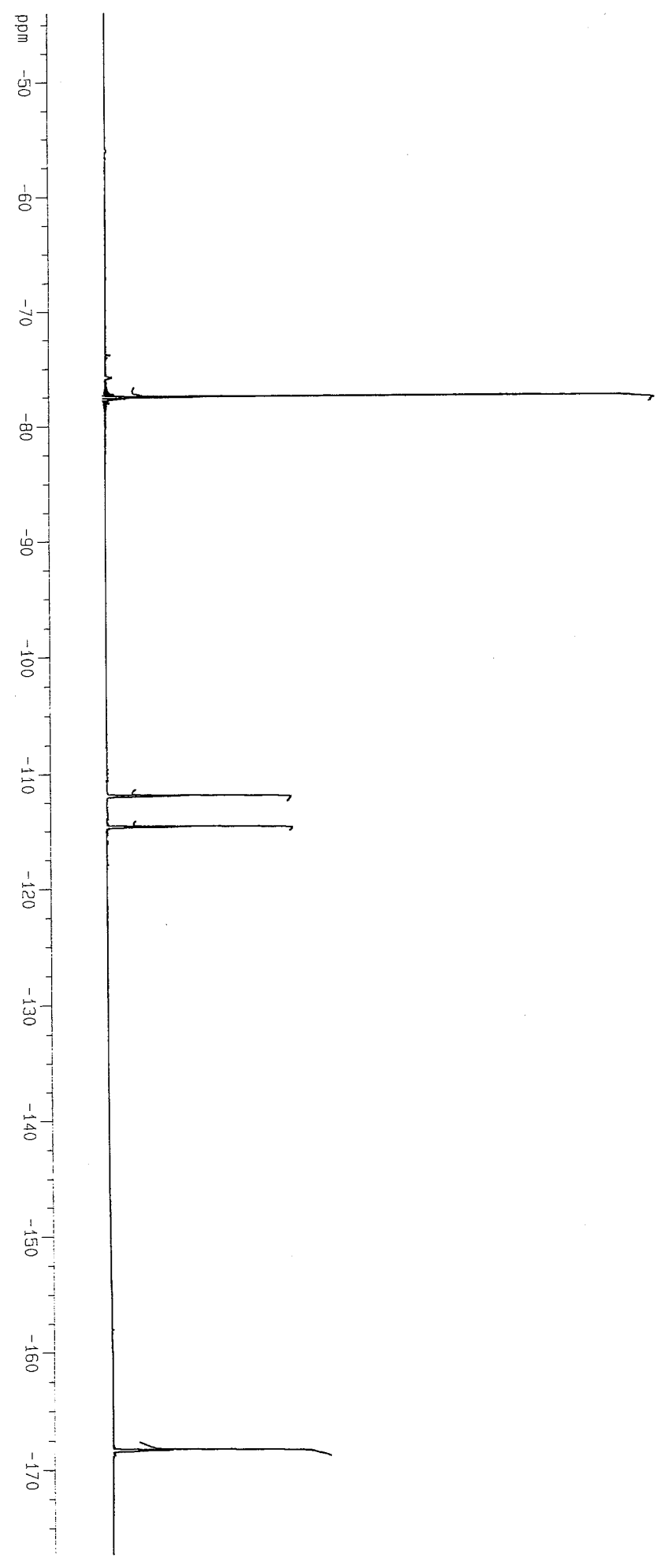

器

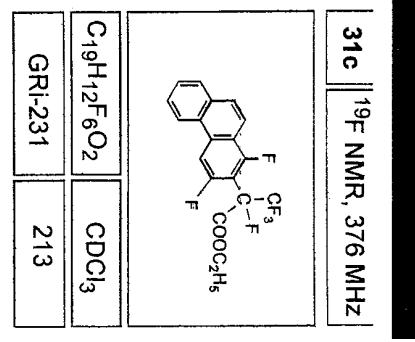



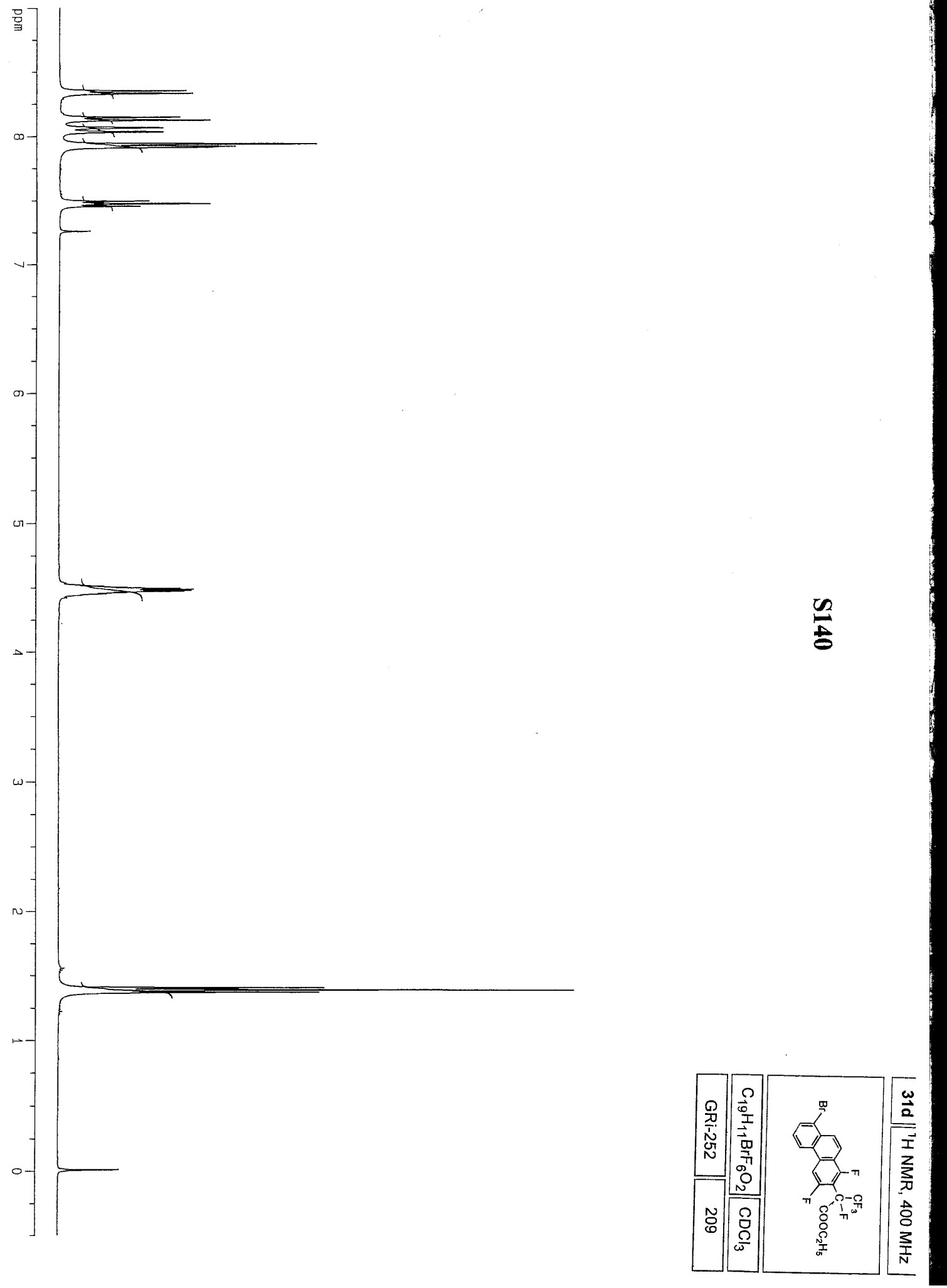


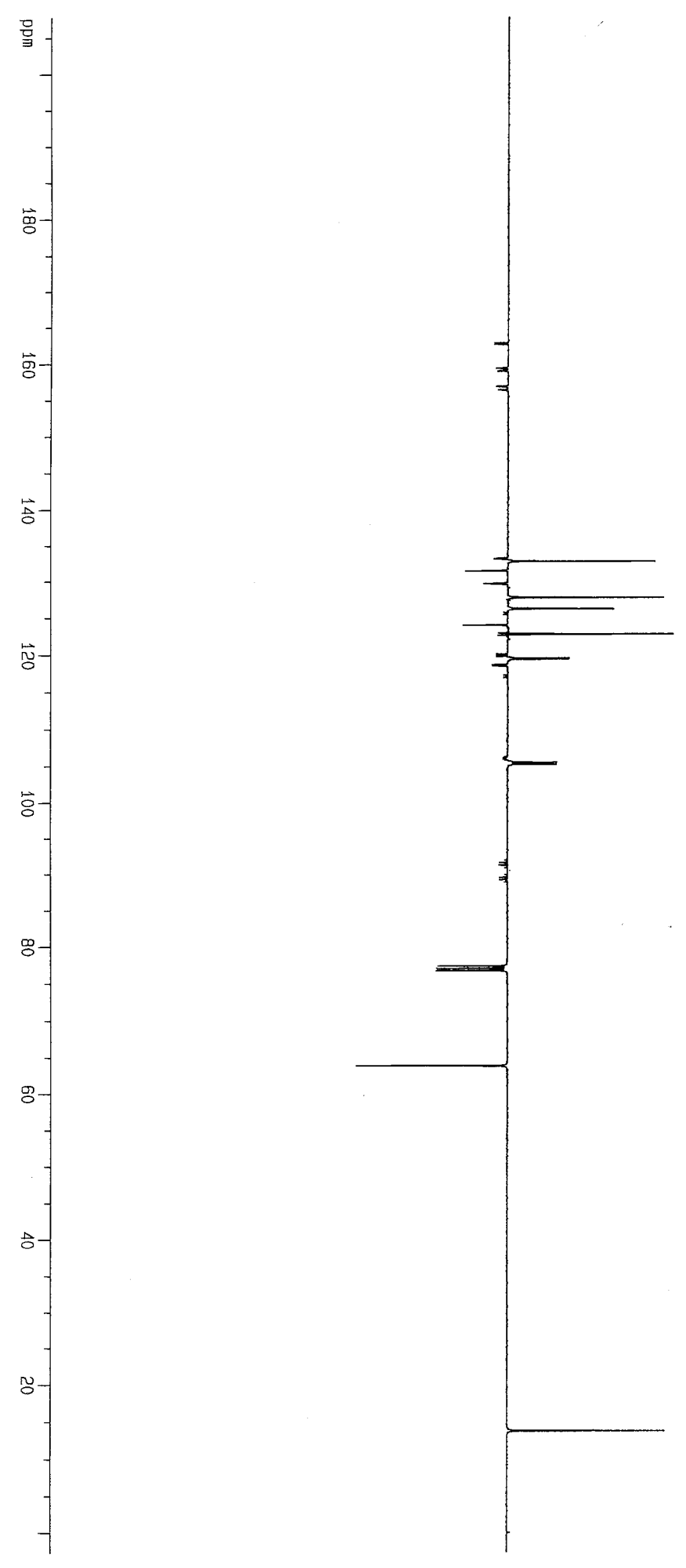

$\stackrel{\mathscr{E}}{E}$

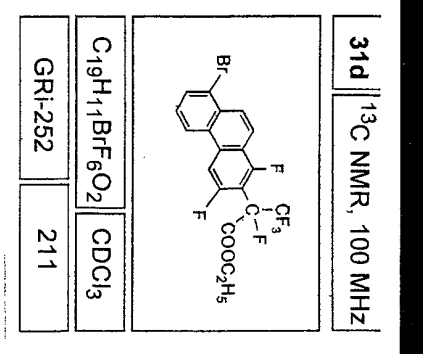




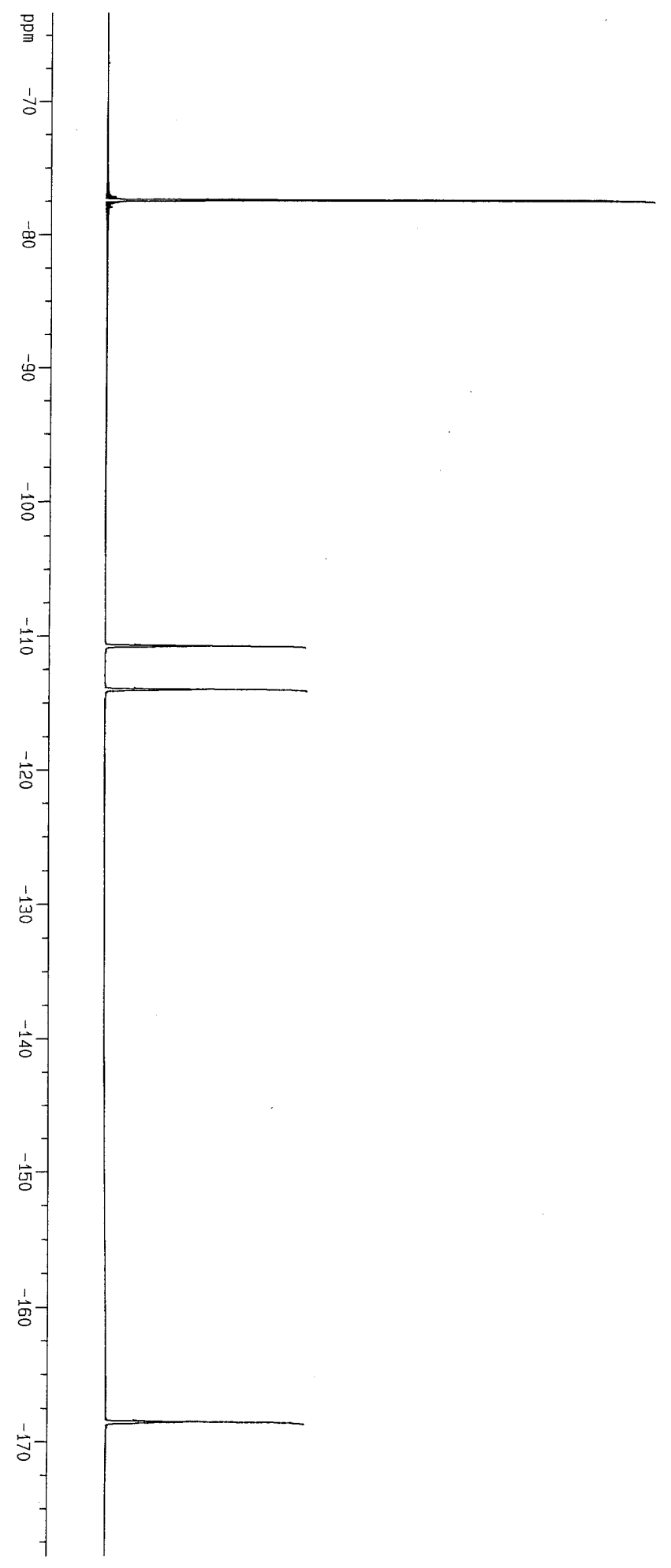

$\frac{\mathscr{E}}{\mathrm{N}}$

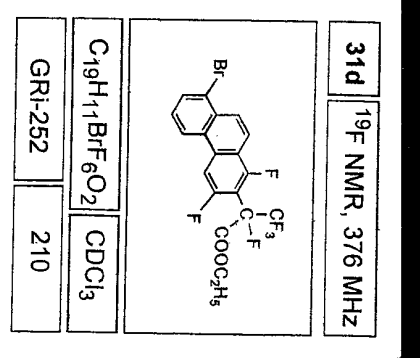

